\title{
Droplet size distribution in condensing flow
}

\author{
R.S.R. Sidin
}


Droplet size distribution in condensing flow

R.S.R. Sidin

Cover: R. Sidin

Thesis University of Twente, Enschede - With summary in Dutch. ISBN 978-90-365-2865-8

Copyright (C) 2009 by R.S.R. Sidin, The Netherlands 


\title{
DROPLET SIZE DISTRIBUTION IN CONDENSING FLOW
}

\section{PROEFSCHRIFT}

\author{
ter verkrijging van \\ de graad van doctor aan de Universiteit Twente, \\ op gezag van de rector magnificus, \\ prof. dr. H. Brinksma, \\ volgens besluit van het College voor Promoties \\ in het openbaar te verdedigen \\ op vrijdag 28 augustus 2009 om 13.00 uur
}

door

Ryan Steeve Rodney Sidin

geboren op 27 maart 1977

te Paramaribo 
Dit proefschrift is goedgekeurd door de promotor:

prof. dr. ir. H.W.M. Hoeijmakers

en de assistent-promotor:

dr. ir. R. Hagmeijer 


\section{TABLE OF CONTENTS}

1 Introduction 1

1.1 Phenomenology of condensation . . . . . . . . . . . . . 1

1.2 Motivation, objective and scope of research . . . . . . . . . . 3

1.3 Thesis outline . . . . . . . . . . . . . . . . . . 4

2 Two-phase mixture model for condensing flow 5

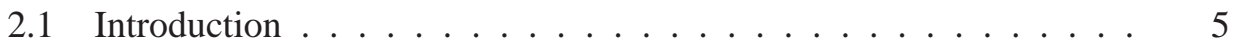

2.2 Notation . . . . . . . . . . . . . . . . 6

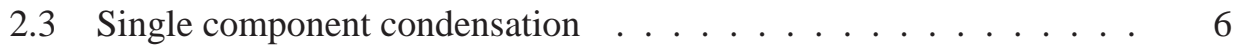

2.3.1 Description of constituent phases . . . . . . . . 6

2.3.2 Droplet mass balance . . . . . . . . . . . . . 8

2.3.3 Droplet momentum balance . . . . . . . . . . . . 10

2.3.4 Droplet energy balance . . . . . . . . . . . . . . . 14

2.3.5 Mixture thermodynamics . . . . . . . . . . . 20

2.3.6 Mixture transport equations . . . . . . . . . . . 24

2.4 Multi-component condensation . . . . . . . . . . . . 31

2.4.1 Description of constituent phases . . . . . . . . . 31

2.4.2 Definition of mass densities and compositions . . . . . . 32

2.4.3 Mixture thermodynamics . . . . . . . . . . . . . 35

2.4 .4 Mixture transport equations . . . . . . . . . 36

3 Evaluation of master equations in condensing flow 37

3.1 Introduction . . . . . . . . . . . . . . . . . 37

3.2 Master equations for the droplet size distribution . . . . . . . . 39

3.2.1 The Kinetic Equation (KE) . . . . . . . . . . . . . . 39

3.2.2 The Fokker-Planck Equation (FPE) . . . . . . . . . . 43

3.2.3 The General Dynamic Equation (GDE) . . . . . . . . . . 45

3.3 Evaluation method . . . . . . . . . . . . . . 48

3.3.1 Description of test cases . . . . . . . . . . . 48 
3.3.2 Numerical methods . . . . . . . . . . . . . . . . . . 49

3.4 Results and discussion $\ldots \ldots \ldots \ldots \ldots$

3.4.1 Equilibrium distributions . . . . . . . . . . . . 53

3.4.2 Test case 1: nucleation pulse experiment . . . . . . . 53

3.4.3 Test case 2: expanding nozzle flow . . . . . . . . . . 59

3.4.4 Sensitivity analysis for the Kinetic Equation . . . . . . . . 69

3.5 Conclusions . . . . . . . . . . . . . . . . 70

4 Reduced models for single component condensation 73

4.1 Introduction . . . . . . . . . . . . . . . . . 73

4.2 Moments and moment equations . . . . . . . . . . . . 75

4.2.1 Moment equations based on the kinetic equation . . . . 75

4.2.2 Moment equations based on the general dynamic equation . 78

4.3 Closure methods for the moment equations . . . . . . . . . 80

4.3.1 Fourier reconstruction . . . . . . . . . . . . 80

4.3.2 Closure Requirements . . . . . . . . . . . . . . 81

4.3.3 Hill's Method of Moments . . . . . . . . . . . . . 84

4.3.4 The quadrature method of moments (QMOM) . . . . . . . 86

4.3.5 The direct quadrature method of moments (DQMOM) . . 96

4.4 Numerical evaluation of closure methods . . . . . . . . . . . . . . . 98

4.4.1 Global procedure . . . . . . . . . . . . . . . 98

4.4.2 Results and discussion . . . . . . . . . . . . . . 98

4.5 Conclusions . . . . . . . . . . . . . . . . 100

5 Evaluation of a flow model with binary condensation 103

5.1 Introduction . . . . . . . . . . . . . . . . . 103

5.2 The kinetic equation for the binary size distribution . . . . . . . . 105

5.3 Mixture properties and fluid dynamics equations . . . . . . . . . 110

5.4 Evaluation procedure . . . . . . . . . . . . . . 113

5.4 .1 Description of test cases . . . . . . . . . . . 113

5.4.2 Numerical methods . . . . . . . . . . . . . . . . . . 114

5.5 Results and discussion . . . . . . . . . . . . . . 118

5.5.1 Test case 1: verification of quasisteady-state and transient

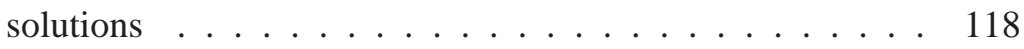

5.5.2 Test case 2: nozzle flow with binary condensation . . . . . 123

5.6 Conclusions ........................ 133

6 Droplet condensation in synthetic turbulence 135

6.1 Introduction . . . . . . . . . . . . . . . . 135

6.2 Physical-mathematical model . . . . . . . . . . . . . . . 139 
6.2.1 Flow field composed of random Fourier modes and related droplet trajectories . . . . . . . . . . . . . . 139

6.2.2 Two-way coupled droplet condensation model . . . . . . . 143

6.2.3 Simplified droplet condensation model . . . . . . . . . 146

6.3 Results . . . . . . . . . . . . . . . . . . . . . . . . 147

6.3.1 Statistics of droplet dispersion . . . . . . . . . . . . 147

6.3.2 Results for simplified condensation model . . . . . . . . . . 148

6.3.3 Results for two-way coupled condensation model . . . . . . 157

6.4 Conclusions . . . . . . . . . . . . . . . . . . . . . 164

7 Conclusions and perspective 167

7.1 Conclusions . . . . . . . . . . . . . . . . . . . . . . 167

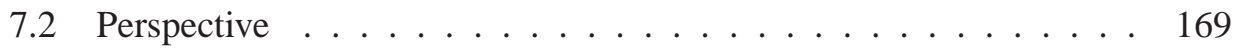

$\begin{array}{lr}\text { References } & 171\end{array}$

$\begin{array}{lr}\text { A Fluid properties } & 183\end{array}$

A.1 Properties of water $\left(\mathrm{H}_{2} \mathrm{O}\right) \ldots \ldots \ldots \ldots \ldots \ldots$

A.2 Properties of heavy water $\left(\mathrm{D}_{2} \mathrm{O}\right) \ldots \ldots \ldots \ldots$

A.3 Properties of nitrogen $\left(\mathrm{N}_{2}\right) \ldots \ldots \ldots \ldots \ldots \ldots$

A.4 Properties of methane $\left(\mathrm{CH}_{4}\right) \ldots \ldots \ldots \ldots \ldots$

A.5 Properties of atmospheric air . . . . . . . . . . . 185

A.6 Properties of ethanol/hexanol mixture . . . . . . . . . . 186

A.7 Properties of ethanol/propanol mixture . . . . . . . . . 186

B Classical nucleation theory and droplet growth 189

$\begin{array}{ll}\text { Summary } & 191\end{array}$

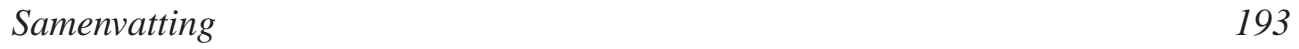

$\begin{array}{ll}\text { Acknowledgment } & 197\end{array}$

About the author 199 



\section{INTRODUCTION}

This chapter gives a brief introduction on the subject of condensing flow. After discussing its relevance with respect to applications in both nature and industry, the scope and objectives of the present investigation are formulated, and an outline of the work is given.

\subsection{Phenomenology of condensation}

The phenomenon of condensation is best known from the extensive cloud systems which dwell in the earth's atmosphere, or from the liquid droplets that form on a cooled surface. Describing this phenomenon as simply "the process of a gas changing to a liquid" [109] does no justice to the complex physics that takes place during this transformation process. This is especially the case for the condensation in atmospheric clouds, in which there is a diverse interaction between various thermal and chemical processes [101].

The initiation of the condensation process is traditionally referred to as nucleation, for which a distinction can be made between (i) homogeneous nucleation, and (ii) heterogeneous nucleation. In homogeneous nucleation, stable clusters of vapor molecules are formed due to random thermal fluctuations in the supersaturated vapor phase, whereas in heterogeneous nucleation, the vapor molecules attach themselves to aerosol particles which act as condensation nuclei. Although the process of cluster formation takes place in any vapor, be it saturated or not, it is only in the supersaturated state that a minimum droplet size exists beyond which the probability of growth is close to certainty. Supersaturation thus typifies the condensation process, and usually, it is quantified by means of the saturation ratio $S$,

$$
S=\frac{p_{v}}{p_{s}(T)},
$$

where $p_{v}$ is the vapor pressure, and $p_{s}(T)$ is the saturation vapor pressure, which is strongly dependent on temperature. The minimum cluster size for droplets to be stable is referred to as the critical size, whereas smaller, and larger clusters are referred to 
as being subcritical, and supercritical, respectively. From a thermodynamic perspective, the critical size can be related to an energy barrier which a cluster must cross, in order to continue its growth to a macroscopic droplet. The number of droplets that cross over this barrier per unit time, and per unit volume of vapor, is referred to as the nucleation rate.

The distinguishing feature between homogeneous and heterogeneous nucleation is that the former generates its own condensation nuclei from the vapor phase, whereas the latter requires foreign particles to activate the condensation process. As the energy barrier associated with the latter is typically much lower than that of the former, this means that for equal nucleation rates, much higher levels of supersaturation in the vapor phase are required for homogeneous nucleation than for heterogeneous nucleation. This is reflected in the dominating presence of heterogeneous nucleation in the natural world, witnessed e.g. in atmospheric clouds (see Figure 1.1.a), whereas homogeneous nucleation is usually found in engineering applications, such as in e.g. high speed aerodynamics (see Figure 1.1.b).

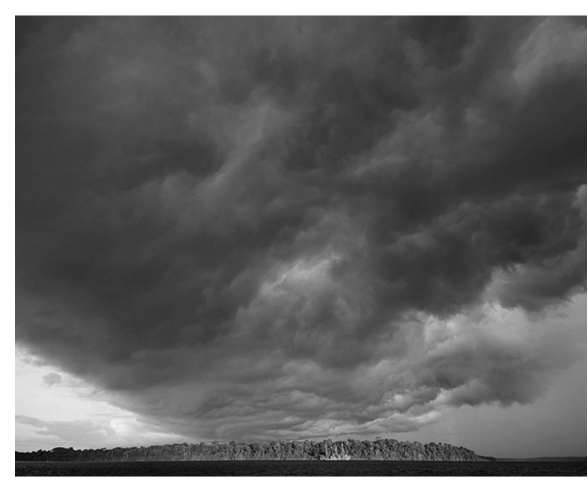

(a)

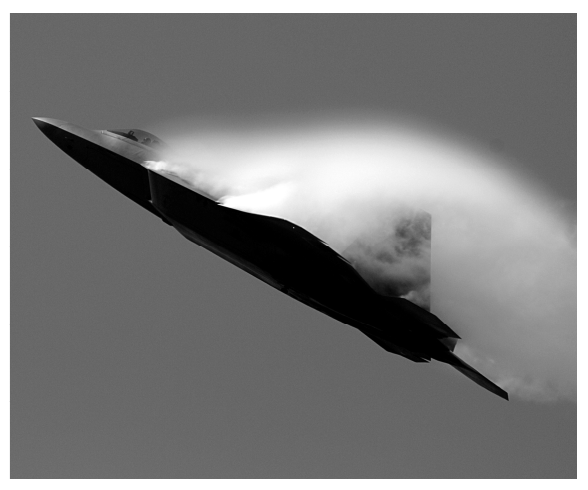

(b)

FIGURE 1.1: Examples of heterogeneous and homogeneous nucleation: (a) rain clouds, initiated by heterogeneous nucleation, gathering above the Suriname River; (b) condensation of water vapor above the wings of an F-22 jet fighter, initiated by homogeneous nucleation (U.S. Air Force photo).

Once stable droplets have been formed, the subsequent condensational growth stage takes place, in which the probability of spontaneous formation or disintegration of droplets is close to zero. The droplets that enter this stage grow in a deterministic fashion due to the steady influx of vapor molecules that is captured from the supersaturated vapor phase. This then results in a decrease of the vapor pressure $p_{v}$, and a corresponding decrease of the supersaturation. Additionally, the transition from vapor to liquid releases latent heat, which causes both the droplet and vapor temperatures 


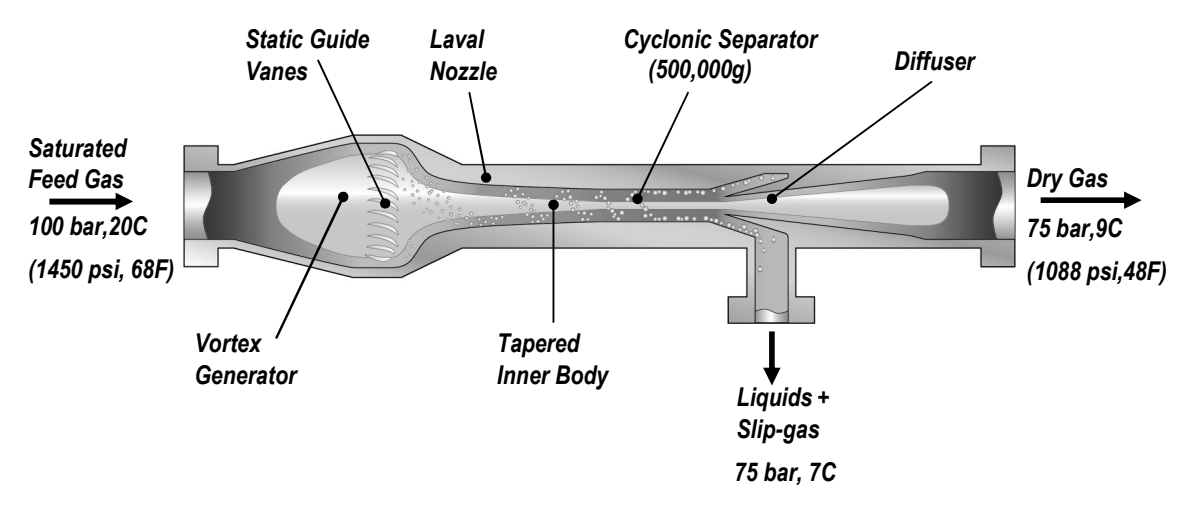

FIGURE 1.2: Schematic layout of the Twister Supersonic Gas Conditioner (image courtesy of Twister B.V.).

to rise. This also results in an increase of the saturation pressure, which, according to Eq. (1.1), means that the saturation level of the vapor is further decreased. As a consequence, the growth rate of droplets steadily decreases, which means that the condensation process is self-regulated.

\subsection{Motivation, objective and scope of research}

The main motivation for this investigation is the Twister Supersonic Gas Conditioner, which is a revolutionary apparatus to remove undesired components from natural gas. The novelty of this device is that it uses condensation to first convert the undesired components to liquid droplets, after which a centrifugal separation step is carried out (see Figure 1.2). The physics that takes place in such devices is very complex, as the flow is both three-dimensional and compressible, and, under certain conditions, even unsteady. Furthermore, it is expected that turbulence could also play a significant role in the condensation and separation of droplets. With respect to the droplets, it is noted that there is a wide variation of scales, as droplets typically start at the nanometer size range $\left(10^{-9} \mathrm{~m}\right)$, and eventually grow to the micrometer-range $\left(10^{-6} \mathrm{~m}\right)$. Within this range of sizes, the mathematical models describing the condensation process, the motion of the droplets, as well as droplet-droplet interactions (e.g. coalescence), can change considerably.

Evidently, one can only focus on a limited number of physical phenomena at a time, which is the strategy that has been followed in previous investigations on the subject (see e.g. [48], [91], [79], and [66]). In the present investigation the focus is on the initial stage of condensation, when droplets are so small that they move like passive tracers. More specifically, the work aims to answer the following questions: 
1. how accurate are existing condensation models in predicting the evolution of the droplet size distribution (DSD) and the flow field variables in rapidly expanding flows?

2. can the balance equation which governs the evolution of the DSD (the socalled master equation) be replaced by computationally more efficient reduced models to approximate condensation effects in rapidly expanding flow with sufficient accuracy?

3. how does the addition of turbulence influence the condensation process in general, and in particular, the evolution of the DSD?

The focus is primarily on the initial stage of condensation, where nucleation plays a central part. The majority of the systems investigated concerns rapidly expanding nozzle flows, for which homogeneous nucleation in an inviscid flow is studied. The influence of turbulence is limited to an investigation of condensing flow in a synthetic turbulent flow field.

\subsection{Thesis outline}

The outline of this thesis is as follows:

- In chapter 2, a two-phase mixture model is presented, in which the focus is on the thermodynamics and governing transport equations for vapor/liquid mixtures typically encountered in flows with single- or multi-component condensation.

- In chapter 3, an evaluation of three master equations is presented, which describe the evolution of the DSD in single-component condensing flow. Furthermore, the sensitivity of the condensation model to physical uncertainties is investigated.

- In chapter 4, reduced models based on the so-called method of moments for single-component condensing flow are evaluated.

- In chapter 5, a model for two-component (binary) condensing nozzle flow is evaluated, and the sensitivity to specific physical uncertainties quantified.

- In chapter 6, an excursion is conducted into the field of turbulent condensing flow, in which a model-system is studied that mimics the condensation in atmospheric systems.

- Finally, the main conclusions of this work are summarized in chapter 7, followed by a review of remaining challenges and suggestions for future research. 


\section{TWO-PHASE MIXTURE MODEL FOR CONDENSING FLOW}

In this chapter the thermodynamic model and transport equations are presented for a two-phase liquid/vapor mixture in transonic condensing flow. First a description is given of the various constituent phases that make up the mixture, after which the governing equations for single component condensing flow are derived. Subsequently, the two-phase fluid model for multi-component condensing flow is presented.

\subsection{Introduction}

Following the common terminology used in the literature [15], [25], [47], condensing flows such as the type discussed in this investigation, can be categorized as two-phase dispersed gas-liquid flows, with two-way coupling between the gaseous carrier phase and the dispersed droplet phase. The classical approach in describing the flows of dispersed two-phase mixtures is to use a so-called two-fluid model, where separate transport equations are derived for the continuous phase and the dispersed phase. In condensing flow, the governing equations are usually written for the entire gas/liquid mixture, rather than for each phase separately. The mixture transport equations are augmented by equations which describe the evolution of the liquid phase, either in terms of a detailed droplet size distribution (DSD) [130], [147], [149], [112], or in terms of its averaged properties via the so-called method of moments (MOM), [1], [42], [45], [68]. In this chapter, the DSD will be employed, and discussion of the MOM will be postponed until chapter 4 . The mixture transport equations, as well as all thermodynamic relationships, are based on the assumption that each fluid element contains representative fractions of all phases, so that a continuum approach may be employed to describe the flow of the mixture. For transonic condensing nozzle flows, homogeneous nucleation yields total droplet number densities of about $10^{18} \mathrm{~m}^{-3}$ [15], corresponding with inter-droplet separation distances of $O\left(10^{-6} \mathrm{~m}\right)$. This is much smaller than the typical length scales of the flow, and therefore, the use of a continuum mixture model is justified.

Contrary to rapidly expanding nozzle flows, the condensation process in atmo- 
spheric clouds is characterized by inter-droplet separation distances of $O\left(10^{-3} \mathrm{~m}\right)$, which are comparable to the smallest scales (Kolmogorov scale) of the turbulent flow in which they are present, [101]. A continuum approach is thus less appropriate for this type of problem, and instead, a combined Eulerian-Lagrangian trajectory model is adopted, which is discussed in chapter 6.

\subsection{Notation}

In the remainder of this thesis, the Einstein summation convention applies to indices $i$ and $j$ only. Each variable related to either the carrier gas, vapor, or a single droplet is assigned the index ' $c$ ', ' $v$ ', or ' $n$ ', respectively. Properties associated with the entire gas phase (i.e., including all carrier gas and vapor components) are assigned the index ' $g$ ', whereas properties associated with the liquid dispersion are assigned the index ' $l$ '. To avoid any confusion regarding the use of the symbol ' $\rho$ ', the following notation convention will be maintained throughout this thesis:

- ' $\rho$ ' refers to a mass density, i.e., the mass of a substance per unit volume;

- ' $\hat{\rho}$ ' refers to a number density, e.g., $\hat{\rho}_{n}$ is the number of droplets of size $n$ per unit volume;

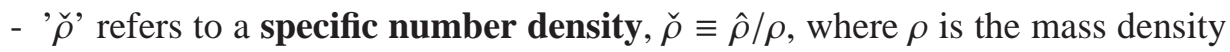
of the liquid/gas mixture. Thus, $\check{\rho}_{n}$ denotes the number of droplets of size $n$ per unit of mixture-mass.

\subsection{Single component condensation}

\subsubsection{Description of constituent phases}

In single component condensation, the mixture generally consists of a dispersed liquid phase and a multi-component gas phase, in which a single condensable vapor is present. All non-condensing constituents of the gas phase are lumped into a single fluid which is referred to as the carrier gas, whereas the term vapor is specifically reserved for the condensable component. For low pressures (i.e., typically a few bars, [66]), the carrier gas does not influence the condensation mechanism directly. Its role is predominantly limited to driving the rapid expansion of the flow so that strong adiabatic cooling and, consequently, high levels of supersaturation can be achieved, which are necessary for homogeneous nucleation to occur.

For rapidly expanding flows, the creation of droplets proceeds via the mechanism of homogeneous nucleation, and therefore, newly generated droplets may start off at the sub-nanometer length scale $\left(O\left(10^{-10} \mathrm{~m}\right)\right)$. Such droplets typically contain less 
than a few tens of molecules, and would, therefore, require a description at the molecular (or microscopic) level. The rapid growth of such droplets results in a poly-disperse system where sizes of $O\left(10^{-7} \mathrm{~m}\right)$ are attained, which is a macroscopic scale. For such large droplets, the droplet temperature is a well defined property, as it is based on an ensemble average of the kinetic fluctuation energy taken over a sufficient number of molecules. For the microscopic droplets, however, the number of molecules in a droplet is so small that the concept of a droplet temperature becomes meaningless. This implies that a hybrid condensation model would actually be required, encompassing a microscopic description for the small droplets and a macroscopic description for the larger ones. Such a model would be very complex, and therefore, condensation models are usually derived from the macroscopic point of view, where it is assumed that the macroscopic model can be extrapolated down to the microscopic length scales. In this thesis, the macroscopic modelling approach will be adopted, because it is the most detailed model that is computationally still feasible for engineering applications.

The droplets resulting from single component condensation consist of a single chemical component, and are usually characterized by size only. The size of a droplet can be expressed in various ways, e.g., by using the number of molecules $n$ which it contains, its mass $m_{n}$, or its equivalent spherical radius $r_{n}$. In general, however, size may not be sufficient to distinguish droplets from one another, as two droplets occupying the same fluid element may be of equal size, but may still differ in shape, temperature, or velocity. For sub-micron droplets, the slip velocity is usually small enough to render the drag force exerted by the carrier gas to be negligibly small compared to capillary forces associated with the surface tension. As a consequence, such droplets maintain a shape that is nearly spherical. Although the term "spherical" is meaningless when a sub-nanometer droplet is considered, in view of the macroscopic modelling approach, even the smallest of droplets are assumed to have a spherical shape. The exchange of energy and momentum between the droplets and the gas phase may cause each droplet to have a distinct temperature $T^{\prime}$ and velocity $\mathbf{v}$, which can be entirely different from the gas temperature $T$ and gas velocity $\mathbf{u}$. Therefore, it is no longer appropriate to use droplet size only for characterization of the dispersed phase. Hence, instead of the mono-variate droplet size distribution, one rather needs to adopt a multi-variate droplet property distribution function (DPD) instead, where the droplet size (e.g., $n$ ), the three velocity components $v_{j}$, and the temperature $T^{\prime}$ constitute the set of independent variables, together with the position vector $\mathbf{x}$ in physical space and time $t$.

To describe the spatio-temporal evolution of the DPD, it is convenient to introduce the seven-dimensional phase space $\Omega_{\xi}$, with the corresponding position vector $\xi$, defined as: $\xi \equiv\left(n, T^{\prime}, \mathbf{v}, \mathbf{x}\right)^{T}$. The corresponding DPD is denoted by the scalar $\Lambda(\xi, t)$. It should be recognized that the droplet size $n$ is an integer $\left(n \in \mathbb{N}^{+}\right)$, whereas the tem- 
perature $T^{\prime}$ and velocity $\mathbf{v}$ vary continuously: $T^{\prime} \in \mathbb{R}^{+}, \mathbf{v} \in \mathbb{R}^{3}$. To emphasize the discrete nature of the distribution in size space, the DPD is replaced by a semi-discrete distribution $\Lambda_{n}\left(T^{\prime}, \mathbf{v}, \mathbf{x}, t\right)$, where $n=2,3, \ldots$ The interpretation given to $\Lambda_{n}\left(T^{\prime}, \mathbf{v}, \mathbf{x}, t\right)$

is conveniently expressed in an integral sense, i.e., $\int_{\Omega_{T}} \int_{\Omega_{v}} \Lambda_{n}\left(T^{\prime}, \mathbf{v}, \mathbf{x}, t\right) d \mathbf{v} d T^{\prime}$ represents the volumetric concentration of droplets of size $n$ at position $\mathbf{x}$ and time $t$, with temperatures lying in the interval $\Omega_{T}$, and velocities in the interval $\Omega_{v}$. Based on this interpretation it is recognized that the number density $\hat{\rho}_{n}$ of $n$-droplets is given by:

$$
\hat{\rho}_{n}(\mathbf{x}, t)=\int_{\mathbb{R}^{+}} \int_{\mathbb{R}^{3}} \Lambda_{n}\left(T^{\prime}, \mathbf{v}, \mathbf{x}, t\right) d \mathbf{v} d T^{\prime} .
$$

The total droplet concentration $N_{l}(\mathbf{x}, t)$ then follows as:

$$
N_{l}(\mathbf{x}, t)=\sum_{n=2}^{\infty} \hat{\rho}_{n},
$$

whereas the total liquid mass density $\rho_{l}(\mathbf{x}, t)$ (i.e., for the whole liquid dispersion) is given by:

$$
\rho_{l}(\mathbf{x}, t)=m_{1} \sum_{n=2}^{\infty} n \hat{\rho}_{n}
$$

with $m_{1}$ the mass of a single vapor molecule. $N_{l}$ and $\rho_{l}$ represent moments of the DPD and provide only a global characterization of the dispersion. In engineering models, knowledge of such moments is frequently deemed sufficient to estimate the impact of condensation on the flow field with reasonable accuracy (see chapter 4). This assumption warrants verification, however, which can only be done by making a direct comparison with a detailed solution for the DPD. Given the large set of independent variables associated with the DPD, it is understandable that only a limited number of investigations have attempted to address this problem. Fortunately, it is possible to reduce the number of independent variables for the DPD, when the scope is limited to the type of condensing flow considered in the present investigation. In order to determine the conditions under which droplet temperature and velocity can be removed from the list of independent variables, it is necessary to consider the exchange of mass, energy, and momentum between the droplets and the ambient phase.

\subsubsection{Droplet mass balance}

The mass balance for a single condensing droplet with control volume $V_{n}$ is given by:

$$
\frac{d m_{n}}{d t}=-\int_{A_{n}} \rho_{v}(\mathbf{x}, t) w_{j}(\mathbf{x}, t) \tilde{n}_{j}(\mathbf{x}, t) d A
$$


where $d m_{n} / d t$ is the mass growth rate of an $n$-droplet, $\mathbf{w}$ represents the velocity of the vapor relative to the moving control surface $A_{n}$, and where $\tilde{\mathbf{n}}$ is the outward unit normal vector on $A_{n}$. The control surface coincides with the droplet surface and follows its movement as the droplet grows (or shrinks). The right-hand side of Eq. (2.4) is the nett condensation (or evaporation) flux, which depends on the droplet surface temperature, the vapor temperature and the local supersaturation. The mass density of the liquid inside the droplet is generally a function of its temperature $T^{\prime}$ and size $n$. The latter dependency will be neglected for droplets consisting of a single component, meaning that the bulk liquid density $\rho_{b}\left(T^{\prime}\right)$ will be used for single-component droplets.

The droplets generated during homogeneous nucleation are usually much smaller in size than the mean free path length $\lambda_{g}$ of the gas/vapor molecules. The Knudsen number, which is defined as:

$$
K n \equiv \frac{\lambda_{g}}{2 r_{n}},
$$

is therefore very large, and thus the condensational growth of droplets takes place in the so-called free molecular (or kinetic) regime.

The mass balance Eq. (2.4) can be rewritten as:

$$
\frac{d m_{n}}{d t}=\left(f_{n}-b_{n}\right) m_{1},
$$

where $f_{n}$ is the rate at which vapor molecules (or monomers) impinge and stick on the droplet surface, whereas $b_{n}$ is the rate at which monomers are emitted from the droplets due to evaporation. In the free molecular regime, $f_{n}$ and $b_{n}$ are expressed as (see e.g., [52]):

$$
\begin{gathered}
f_{n}=f_{n}\left(T, \rho_{v}\right)=\zeta_{n}^{v} \frac{\rho_{v}}{m_{1}} \sqrt{T}, \\
b_{n}=b_{n}\left(T^{\prime}\right)=\zeta_{n-1}^{v} \frac{\rho_{v, n}^{s}\left(T^{\prime}\right)}{m_{1}} \sqrt{T^{\prime}},
\end{gathered}
$$

with $\zeta_{n}^{v}$ given by:

$$
\zeta_{n}^{v}=a_{1}\left(n^{1 / 3}+1\right)^{2} \sqrt{\frac{n+1}{n}} \sqrt{\frac{k_{B}}{2 \pi m_{1}}},
$$

with $k_{B}$ the Boltzmann constant, $a_{1}$ the effective molecular surface area, and $\rho_{v, n}^{s}$ the saturated vapor density over the curved surface of a droplet of size $n$ and temperature $T^{\prime}$, [52]. The impingement rate $f_{n}$ thus depends on the gas/vapor temperature and mass density, whereas the evaporation rate $b_{n}$ depends on the droplet properties only.

During droplet growth, the expression for the growth rate changes, as the condensation process gradually shifts from the free molecular regime $(K n \gg 1)$ towards the continuum regime $(K n \ll 1)$. Throughout the years, several models have been developed, which attempt to describe the growth rate in both the kinetic and continuum 
regimes, as well as in the transition regime between these two extremes. Most notable are the models presented by Gyarmathy in [39], and by Young in [147], [148]. The interested reader is referred to these works for a detailed exposition.

\subsubsection{Droplet momentum balance}

For a single condensing droplet moving within a gaseous carrier phase, the momentum balance is given by:

$$
\frac{d m_{n}}{d t} v_{i}+m_{n} \frac{d v_{i}}{d t}=F_{i}-\int_{A_{n}} \rho_{v}(\mathbf{x}, t)\left(v_{i}^{\prime}(\mathbf{x}, t)+w_{i}(\mathbf{x}, t)\right) w_{j}(\mathbf{x}, t) \tilde{n}_{j}(\mathbf{x}, t) d A
$$

where $\mathbf{F}$ is the force exerted by the carrier fluid on the droplet, $\mathbf{v}$ is the velocity of the droplet, i.e.,

$$
\mathbf{v}=\mathbf{v}(t)=\frac{d \mathbf{x}^{\prime}(t)}{d t}
$$

with $\mathbf{x}^{\prime}(t)$ the droplet position, and where $\mathbf{v}^{\prime}(\mathbf{x}, t)$ is the velocity of the liquid phase at the droplet surface. For sub-micron droplets moving at low slip velocities, i.e., $\|\mathbf{v}-\mathbf{u}\| /\|\mathbf{u}\| \ll 1$, with $\mathbf{u}$ the gas velocity, the shape is nearly spherical and the internal recirculation flow is negligibly small. As a consequence, the droplet momentum balance can be expressed as:

$\frac{d m_{n}}{d t} v_{i}+m_{n} \frac{d v_{i}}{d t}=F_{i}-v_{i} \int_{A_{n}} \rho_{v}(\mathbf{x}, t) w_{j}(\mathbf{x}, t) \tilde{n}_{j}(\mathbf{x}, t) d A-\int_{A_{n}} \rho_{v}(\mathbf{x}, t) w_{i}(\mathbf{x}, t) w_{j}(\mathbf{x}, t) \tilde{n}_{j}(\mathbf{x}, t) d A$,

and additionally, the force $\mathbf{F}$ can be calculated using one of the many semi-empirical expressions available for the drag experienced by rigid spherical particles [22]. From the mass balance in Eq. (2.4) it is recognized that the first term on the left-hand side of Eq. (2.12) cancels the second term on the right-hand side, which leads to the more common form of the momentum balance:

$$
m_{n} \frac{d v_{i}}{d t}=F_{i}-\int_{A_{n}} \rho_{v}(\mathbf{x}, t) w_{i}(\mathbf{x}, t) w_{j}(\mathbf{x}, t) \tilde{n}_{j}(\mathbf{x}, t) d A .
$$

The last term on the right-hand side of Eq. (2.13) is the so-called Stefan flux, which is a thrust force generated by the vapor flow towards the droplet [111]. For small slip velocities, vapor condensation proceeds in a near symmetrical fashion, so that the Stefan flux can be neglected compared to the remaining terms in Eq. (2.13). As a consequence, the droplet momentum balance reduces to that for a rigid particle:

$$
m_{n} \frac{d v_{i}}{d t}=F_{i}
$$


The equation of motion for rigid particles has been the subject of many investigations, and the most notable is the seminal work by Maxey and Riley in [72]. They present the particle momentum balance as follows:

$$
m_{n} \frac{d v_{i}}{d t}=F_{b, i}+F_{p, i}+F_{a, i}+F_{v, i}+F_{B, i}
$$

where:

$$
F_{b, i}=\left(m_{n}-m_{g}\right) g_{i}
$$

is the buoyancy force, with $\mathbf{g}$ the gravitational acceleration vector, and $m_{g}$ the mass of carrier fluid displaced by the droplet;

$$
F_{p, i}=m_{g} \frac{D u_{i}}{D t}\left(\mathbf{x}^{\prime}(t), t\right)
$$

is the pressure gradient force, with $D / D t$ the material derivative;

$$
F_{a, i}=-\frac{1}{2} m_{g} \frac{d}{d t}\left[v_{i}(t)-u_{i}\left(\mathbf{x}^{\prime}(t), t\right)-\frac{1}{10} \nabla^{2} u_{i}\left(\mathbf{x}^{\prime}(t), t\right)\right]
$$

is the added mass term;

$$
F_{v, i}=-6 \pi r_{n} \rho_{g} v_{g}\left[v_{i}(t)-u_{i}\left(\mathbf{x}^{\prime}(t), t\right)-\frac{r_{n}^{2}}{6} \nabla^{2} u_{i}\left(\mathbf{x}^{\prime}(t), t\right)\right]
$$

is the viscous drag force;

$$
F_{B, i}=-6 \pi r_{n}^{2} \rho_{g} v_{g} \int_{0}^{t} \frac{d}{d \tau}\left(v_{i}(t)-u_{i}\left(\mathbf{x}^{\prime}(t), t\right)-\frac{r_{n}^{2}}{6} \nabla^{2} u_{i}\left(\mathbf{x}^{\prime}(t), t\right)\right)(\pi(t-\tau))^{-1 / 2} v_{g} d \tau
$$

is the Basset-history term. Calculation of these forces requires the gas density $\rho_{g}$ and kinematic viscosity $v_{g}$ at the droplet position $\mathbf{x}^{\prime}(t)$, and the droplet radius $r_{n}$. The occurrence of the $\nabla^{2} u_{i}$ - term in Eqs. (2.18), (2.19), and (2.20) accounts for the variation of the flow field on the length scale of the particle, usually denoted as the Faxen correction [26]. Apparently, this correction will be of minor importance when the particle is much smaller than the typical length scales of the flow.

The foregoing expressions are valid for a continuum field surrounding the particle. When the droplets are much smaller than the mean free path length of the gas phase, i.e., $K n \gg 1$, rarefaction effects need to be taken into account. This is accomplished by multiplying the particle drag force with the so-called Cunningham correction factor, which only depends on $K n$. Details on this correction and its limitations are discussed extensively in amongst others [26], and are not repeated here.

To assess the importance of each of the force contributions, Eq. (2.15) is cast into non-dimensional form, using a velocity scale $U_{0}$, length scale $L_{0}$, and time scale 
$\tau_{0}=L_{0} / U_{0}$, associated with the carrier gas flow. After some manipulations, the following dimensionless form of the droplet momentum equation is obtained:

$$
\begin{gathered}
\frac{d \tilde{v}_{i}}{d t}=\left(1-\frac{\rho_{g}}{\rho_{b}}\right) \frac{g_{i}}{g} \frac{1}{\operatorname{Fr}^{2}}+\frac{\rho_{g}}{\rho_{b}} \frac{D \tilde{u}_{i}}{D \tilde{t}}-\frac{\rho_{g}}{\rho_{b}} \frac{1}{2} \frac{d}{d \tilde{t}}\left(\tilde{v}_{i}-\tilde{u}_{i}-\frac{\tilde{r}_{n}^{2}}{10} \tilde{\nabla}^{2} \tilde{u}_{i}\right)- \\
\frac{1}{\mathrm{St}}\left(\tilde{v}_{i}-\tilde{u}_{i}-\frac{\tilde{r}_{n}^{2}}{6} \tilde{\nabla}^{2} \tilde{u}_{i}\right)-\frac{\tilde{r}_{n}}{\operatorname{Re}^{1 / 2} \mathrm{St}} \int_{0}^{\tilde{t}} \frac{d}{d \tau}\left(\tilde{v}_{i}-\tilde{u}_{i}-\frac{\tilde{r}_{n}^{2}}{6} \tilde{\nabla}^{2} \tilde{u}_{i}\right)(\pi(\tilde{t}-\tau))^{-1 / 2} d \tau
\end{gathered}
$$

where $g \equiv\|\mathbf{g}\|$, and the dimensionless version of each variable is indicated by a tilde, e.g., $\tilde{r}_{n}=r_{n} / L_{0}$. The preceding equation contains the Froude number

$$
\operatorname{Fr}=\frac{U_{0}}{\sqrt{g L_{0}}}
$$

the free stream Reynolds number

$$
\operatorname{Re}=\frac{U_{0} L_{0}}{v_{g}}
$$

and the Stokes number

$$
\mathrm{St}=\frac{\tau_{v}}{\tau_{0}}
$$

with the particle momentum relaxation time $\tau_{v}$ given by:

$$
\tau_{v}=\frac{2}{9} \frac{\rho_{b}}{\rho_{g}} \frac{r_{n}^{2}}{v_{g}}
$$

In the test cases that will be considered in this thesis, the flow is usually transonic at temperatures between $200 \mathrm{~K}$ and $300 \mathrm{~K}$, which means that for the typical Mach numbers of $M \sim 1$, the reference velocity will be $U_{0} \sim 10^{2} \mathrm{~m} / \mathrm{s}$. For the nozzle flows considered in this thesis, the typical length scales associated with the flow vary within the range $L_{0} \sim 10^{-2}-10^{-1} \mathrm{~m}$ (see chapter 3), which means that $\mathrm{Fr} \sim 10^{2} \gg 1$. Buoyancy forces are therefore not important, and thus the first term on the right-hand side of Eq. (2.21) can be neglected. The mass density of the liquid in the droplets is much higher than that of the carrier gas: $\rho_{g} / \rho_{b} \sim 10^{-3} \ll 1$, which allows one to neglect the second and third term in Eq. (2.21). As mentioned previously, droplets observed in transonic condensing flows typically fall within the size range $10^{-10}<$ $r_{n}<10^{-7} \mathrm{~m}$, so that $\tilde{r}_{n} \leq 10^{-5} \ll 1$. As a consequence, the last term in Eq. (2.21) can be neglected, as well as the Faxen correction in the third term. The resulting dimensionless form of the droplet momentum balance in transonic condensing flow is thus reduced to:

$$
\frac{d \tilde{v}_{i}}{d \tilde{t}}=\frac{1}{\mathrm{St}}\left(\tilde{u}_{i}-\tilde{v}_{i}\right)
$$


and its corresponding fully dimensional form is given by:

$$
\frac{d v_{i}}{d t}(t)=\frac{1}{\tau_{v}\left(\mathbf{x}^{\prime}(t), t\right)}\left(u_{i}\left(\mathbf{x}^{\prime}(t), t\right)-v_{i}(t)\right) .
$$

The influence of the Stokes number is best revealed when Eq. (2.23) is rewritten as:

$$
\tilde{v}_{i}=\tilde{u}_{i}-\mathrm{St} \frac{d \tilde{v}_{i}}{d \tilde{t}}
$$

For $\mathrm{St} \rightarrow 0$, the droplet follows the carrier fluid, since $\tilde{v}_{i} \rightarrow \tilde{u}_{i}$.

When St $<1$, and droplet inertia is not negligible, an estimate can be made of the droplet velocity using a so-called algebraic slip model. For this purpose, Eq. (2.25) is first differentiated with respect to time, which, after some further manipulations, yields:

$$
\frac{d \tilde{v}_{i}}{d \tilde{t}}=\frac{\mathrm{St}}{1-\frac{d \mathrm{St}}{d t}}\left[\frac{d \tilde{u}_{i}}{d \tilde{t}}-\mathrm{St} \frac{d^{2} \tilde{v}_{i}}{d \tilde{t}^{2}}\right] .
$$

Noting that $\tilde{u}_{i}=\tilde{u}_{i}\left(\tilde{\mathbf{x}}^{\prime}(\tilde{t}), \tilde{t}\right)$ in Eq. (2.25), its time-derivative can be expanded as:

$$
\frac{d \tilde{u}_{i}}{d \tilde{t}}\left(\tilde{\mathbf{x}}^{\prime}(\tilde{t}), \tilde{t}\right)=\tilde{v}_{j} \frac{\partial \tilde{u}_{i}}{\partial \tilde{x}_{j}}\left(\tilde{\mathbf{x}}^{\prime}(\tilde{t}), \tilde{t}\right)+\frac{\partial \tilde{u}_{i}}{\partial \tilde{t}}\left(\tilde{\mathbf{x}}^{\prime}(\tilde{t}), \tilde{t}\right) .
$$

Substituting Eq. (2.25) for $\tilde{v}_{j}$ in Eq. (2.27), one obtains:

$$
\frac{d \tilde{u}_{i}}{d \tilde{t}}\left(\tilde{\mathbf{x}}^{\prime}(\tilde{t}), \tilde{t}\right)=\frac{D \tilde{u}_{i}}{D \tilde{t}}\left(\tilde{\mathbf{x}}^{\prime}(\tilde{t}), \tilde{t}\right)-\mathrm{St} \frac{d \tilde{v}_{j}}{d \tilde{t}} \frac{\partial \tilde{u}_{i}}{\partial \tilde{x}_{j}}\left(\tilde{\mathbf{x}}^{\prime}(\tilde{t}), \tilde{t}\right),
$$

where $D / D \tilde{t}$ denotes the dimensionless material derivative. Substitution of Eqs. (2.26) and (2.28) in Eq. (2.25) then yields:

$$
\tilde{v}_{i}=\tilde{u}_{i}-\frac{\mathrm{St}}{1-\frac{d \mathrm{St}}{d t}}\left[\frac{D \tilde{u}_{i}}{D \tilde{t}}-\mathrm{St} \frac{d \tilde{v}_{j}}{d \tilde{t}} \frac{\partial \tilde{u}_{i}}{\partial \tilde{x}_{j}}-\mathrm{St} \frac{d^{2} \tilde{v}_{i}}{d \tilde{t}^{2}}\right] .
$$

Neglecting the terms which are quadratic in St then results in the following estimate $\tilde{\mathbf{v}}_{n}$ for the dimensionless droplet velocity:

$$
\tilde{v}_{n, i}=\tilde{u}_{i}-\frac{\mathrm{St}}{1-\frac{d \mathrm{St}}{d t}} \frac{D \tilde{u}_{i}}{D \tilde{t}}
$$

with its fully-dimensional analogue given by:

$$
v_{n, i}=u_{i}-\frac{\tau_{v}}{1-\frac{d \tau_{v}}{d t}} \frac{D u_{i}}{D t}
$$


Eq. (2.31) allows the droplet velocity to be calculated from the velocity field at the droplet position, and the droplet size. Using this algebraic model, the droplet velocity can now be removed from the list of independent variables associated with the droplet property distribution function, and thus $\Lambda_{n}\left(T^{\prime}, \mathbf{v}, \mathbf{x}, t\right)$ can be replaced by $\Lambda_{n}^{\prime}\left(T^{\prime}, \mathbf{x}, t\right)$, where:

$$
\Lambda_{n}^{\prime}\left(T^{\prime}, \mathbf{x}, t\right)=\int_{\mathbb{R}^{3}} \Lambda_{n}\left(T^{\prime}, \mathbf{v}, \mathbf{x}, t\right) d \mathbf{v} .
$$

The Stokes number associated with the test cases considered in the remainder of this thesis are typically smaller than $10^{-2}$. Therefore, droplet slip is neglected entirely, so that: $\mathbf{v}_{n}=\mathbf{u}$.

\subsubsection{Droplet energy balance}

In the free molecular flow regime, the droplet exchanges energy with the gas/vapor phase due to impingement and reflection of gas- and vapor molecules, evaporation, and the expansion work done by the growing droplet. The change in internal energy $\left(E_{n}\right)$ of a single isolated droplet of size $n$ is given by:

$$
\frac{d E_{n}}{d t}=\dot{q}_{v}^{c}-\dot{q}_{v}^{e}+\dot{q}_{c}+p \dot{V}_{n}
$$

The incoming heat flux associated with the impinging and reflecting vapor molecules is denoted by $\dot{q}_{v}^{c}$, whereas $\dot{q}_{v}^{e}$ represents the outgoing heat flux due to evaporation. The heat flux caused by impingement and reflection of the carrier gas molecules is represented by $\dot{q}_{c}$, and $p \dot{V}_{n}$ is the expansion work done by the growing droplet, per unit time.

The droplet energy can be written as a sum of a bulk internal energy and a surface energy [149]:

$$
E_{n}=\int_{V_{n}} \rho_{b} e_{b} d V+\int_{A_{n}} \psi d A,
$$

where $e_{b}$ is the specific internal energy of the internal bulk liquid, and where $\psi=$ $\psi\left(T^{\prime}\right)$ is the specific surface energy. The latter is given by:

$$
\psi=\sigma-T^{\prime} \frac{d \sigma}{d T^{\prime}},
$$

with $\sigma=\sigma\left(T^{\prime}\right)$ denoting the surface free energy (or surface tension) [149]. The specific bulk internal energy $e_{b}$ is equal to:

$$
e_{b}=h_{b}-\frac{p_{n}}{\rho_{b}}
$$


with $h_{b}=$ the specific enthalpy of the internal liquid phase, and $p_{n}$ the pressure within the droplet. Assuming that the droplet is spherical, and that mechanical equilibrium is maintained, the internal pressure relates to the external gas pressure $p$ as:

$$
p_{n}=p+\frac{2 \sigma}{r_{n}} .
$$

Using the definition of the latent heat of vaporization for bulk incompressible liquid:

$$
L_{b}=L_{b}\left(T^{\prime}\right) \equiv h_{v}^{s}\left(T^{\prime}\right)-h_{b}\left(T^{\prime}\right),
$$

where $h_{v}^{s}$ is the specific vapor enthalpy at saturation $(S=1)$, and by neglecting gradients within the droplet, the preceding equations allow the droplet energy (Eq. (2.33)) to be expressed as:

$$
E_{n}=m_{n} h_{v}^{s}-m_{n} L_{b}-p V_{n}-\frac{2 \sigma}{r_{n}} V_{n}+\left(\sigma-T^{\prime} \frac{d \sigma}{d T^{\prime}}\right) 4 \pi r_{n}^{2}
$$

Introducing a size dependent latent heat of vaporization $L_{n}=L_{n}\left(T^{\prime}\right)$ :

$$
L_{n}\left(T^{\prime}\right) \equiv L_{b}+\frac{2 \sigma}{r_{n} \rho_{b}}-\frac{3}{r_{n} \rho_{b}}\left(\sigma-T^{\prime} \frac{d \sigma}{d T^{\prime}}\right),
$$

this leads to:

$$
E_{n}=m_{n}\left(h_{v}^{s}-L_{n}\right)-p V_{n}
$$

For droplets that can be treated as macroscopic entities (say $n>10^{3}$ ), calculations based on Eq. (2.39) show that the difference between $L_{b}$ and $L_{n}$ is usually negligibly small. Taking the example of water droplets, it is found that the relative difference between $L_{b}$ and $L_{n}$ is typically of the order of a few percent, as shown in Figure 2.1.a. For very small droplets $(n<100)$, the macroscopic model in Eq. (2.39) is no longer valid, and therefore, an approach at the molecular level is necessary to formulate $L_{n}$. For such sizes, $L_{n}$ is interpreted as the specific heat of formation associated with a reaction in which $n$ initially separated vapor molecules end up as a single $n$-sized cluster.

Figure 2.1.b shows the variation of $L_{n}$ with $n$, as obtained from molecular theory, and from mass-spectroscopic measurements by Sukhodub et al. [117], along with the predictions obtained with Eq. (2.39) for water droplets at a temperature of $T^{\prime}=300 \mathrm{~K}$. It is noted that for small droplets $(n<10)$, the differences between the predictions by the macroscopic model (Eq. (2.39)) and those by molecular theory are very large, as one might expect. The data given by Sukhodub et al. suggest that the latent heat of condensation can be described by an empirical function of the form:

$$
L_{n}\left(T^{\prime}\right)=L_{b}\left(T^{\prime}\right) \beta(n)
$$




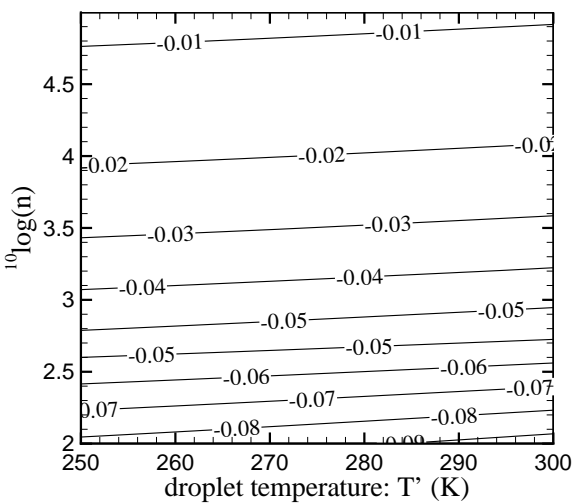

(a)

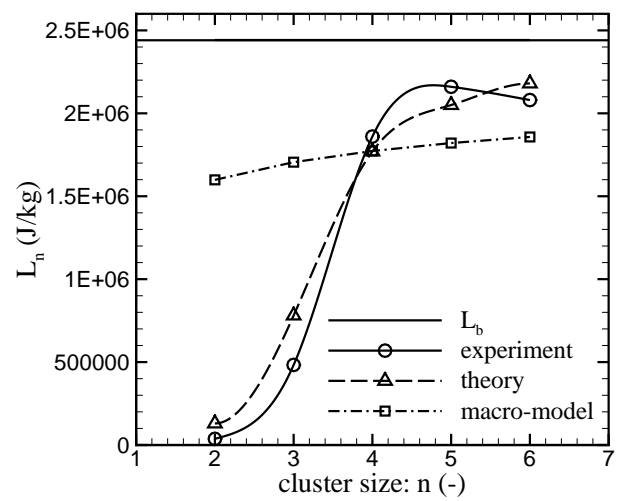

(b)

FIGURE 2.1: Size dependent latent heat of condensation for water droplets: (a) isolines for the relative difference $\left[L_{n}\left(T^{\prime}\right)-L_{b}\left(T^{\prime}\right)\right] / L_{b}\left(T^{\prime}\right)$ for various droplet sizes and temperatures, calculated by means of Eq. (2.39) and the properties of water in appendix A; (b) latent heat data from mass-spectroscopic measurements and theoretical predictions by Sukhodub et al., compared to calculations obtained with the macroscopic model (Eq. (2.39)) for $T^{\prime}=300 \mathrm{~K}$, [117].

where:

$$
\beta(n)=1-\left(1-c_{0}\right) \exp \left(\frac{2-n}{c_{1}}\right),
$$

with $c_{0}=L_{2} / L_{b}$, and $c_{1}$ a parameter that controls the variation in $L_{n}$.

Using Eq. (2.40), the energy balance Eq. (2.32) can now be rewritten. By differentiating Eq. (2.40) with respect to time, and assuming that changes in $E_{n}$ due to temporal variations in pressure can be neglected, one obtains:

$$
\frac{d E_{n}}{d t}=\dot{m}_{n}\left(h_{v}^{s}-L_{n}-n \frac{\partial L_{n}}{\partial n}\right)+\dot{T}^{\prime} m_{n}\left(\frac{d h_{v}^{s}}{d T^{\prime}}-\frac{\partial L_{n}}{\partial T^{\prime}}\right)-p \dot{V}_{n},
$$

where $\dot{m}_{n}=d m_{n} / d t$. Subsequently, the droplet energy balance Eq. (2.32) can be rewritten as:

$$
\dot{m}_{n}\left(h_{v}^{s}-L_{n}-n \frac{\partial L_{n}}{\partial n}\right)+\dot{T}^{\prime} m_{n}\left(\frac{d h_{v}^{s}}{d T^{\prime}}-\frac{\partial L_{n}}{\partial T^{\prime}}\right)=\dot{q}_{v}^{c}-\dot{q}_{v}^{e}+\dot{q}_{c} .
$$

To compute the energy fluxes on the right-hand side of Eq. (2.44), it is assumed that (i) all vapor and carrier gas molecules reflecting from the droplet surface equilibrate to the droplet temperature, and that (ii) the velocities of the vapor and carrier gas molecules satisfy a Maxwellian velocity distribution. The latter assumption is somewhat questionable, as the rapidly expanding flow causes the vapor and carrier gas to be in a non-equilibrium state. The impact of this assumption on the condensation process is not addressed here, since it is beyond the scope of the present thesis. 
Based on the preceding assumptions, the energy flux associated with the impinging and reflecting vapor molecules is given by:

$$
\dot{q}_{v}^{c}=\frac{f_{n} m_{1}}{\alpha_{n}}\left(C_{p, v}-\frac{R_{v}}{2}\right)\left[T-\left(1-\alpha_{n}\right) T^{\prime}\right]
$$

where $\alpha_{n}$ is the fraction of impinging vapor molecules that stick to the droplet, usually referred to as the sticking probability. $C_{p, i}$ is the specific heat at constant pressure for component $i$, and $R_{i}$ is the corresponding specific gas constant. It is noted that Eq. (2.45) is obtained by integrating the energy flux density associated with the Maxwellian velocity distribution over the half-infinite velocity space, which explains why the term within brackets is $C_{p, v}-\frac{1}{2} R_{v}$, instead of $C_{p, v}-R_{v}=C_{v, v}$, [66]. Similarly, the energy flux associated with the evaporating vapor molecules is given by:

$$
\dot{q}_{v}^{e}=b_{n} m_{1}\left(C_{p, v}-\frac{R_{v}}{2}\right) T^{\prime}
$$

The energy flux associated with the impinging and reflecting molecules of carrier gas component $k$ is expressed as:

$$
\dot{q}_{c, k}=\zeta_{n}^{c, k} \sqrt{T} \rho_{c, k}\left(C_{p, k}-\frac{R_{k}}{2}\right)\left[T-T^{\prime}\right]
$$

where $\rho_{c, k}$ is the mass density of carrier gas component $k$, and where the factor $\zeta_{n}^{c, k}$ is given by:

$$
\zeta_{n}^{c, k}=a_{1}\left(n^{1 / 3}+\frac{r_{c, k}}{r_{1}}\right)^{2} \sqrt{\frac{1+\frac{m_{1}}{m_{c, k}} n}{n}} \sqrt{\frac{k_{B}}{2 \pi m_{1}}} .
$$

Here, $m_{c, k}$, and $r_{c, k}$, are the mass and effective radius of a single molecule of component $k$ of the carrier gas, respectively. Finally, the total energy flux removed by the carrier gas is then given by:

$$
\dot{q}_{c}=\sum_{k=1}^{N_{c}} \dot{q}_{c, k}
$$

where $N_{c}$ is the number of carrier gas components.

With these expressions for the energy fluxes $\dot{q}_{v}^{c}, \dot{q}_{v}^{e}$, and $\dot{q}_{c}$, viz. Eq. (2.45), Eq. (2.46), and Eq. (2.49), the energy balance Eq. (2.44) can be used to obtain the following expression for the time-derivative of the droplet temperature:

$$
\dot{T}^{\prime}=-\frac{1}{\tau_{T}}\left(T^{\prime}-T_{n}\right)
$$


In this expression, the thermal relaxation time $\tau_{T}$ for the droplet is given by:

$$
\tau_{T}=\frac{m_{n}\left[\frac{d h_{v}^{s}}{d T^{\prime}}-\frac{\partial L_{n}}{\partial T^{\prime}}\right]}{\left[\zeta_{n}^{v} \frac{1-\alpha_{n}}{\alpha_{n}}\left(C_{p, v}-\frac{R_{v}}{2}\right) \rho_{v}+\sum_{k=1}^{N_{c}} \zeta_{n}^{c, k}\left(C_{p, k}-\frac{R_{k}}{2}\right) \rho_{c, k}\right] \sqrt{T}+\zeta_{n-1}^{v}\left(C_{p, v}-\frac{R_{v}}{2}\right) \rho_{v, n}^{s} \sqrt{T^{\prime}}}
$$

and $T_{n}$ is the so-called wet bulb temperature, which is implicitly given by:

$$
T_{n}=\frac{\left[\frac{1}{\alpha_{n}}+\sum_{k=1}^{N_{c}} \frac{\zeta_{n}^{c, k}}{\zeta_{n}^{v}} \frac{\rho_{c, k}}{\rho_{v}} \frac{C_{p, k}-\frac{R_{k}}{2}}{C_{p, v}-\frac{R_{v}}{2}}\right] T-\left[1-\frac{\zeta_{n-1}^{v}}{\zeta_{n}^{v}} S_{n}\left(T_{n}\right) \sqrt{\left.\frac{T_{n}}{T}\right] \frac{h_{v}^{s}\left(T_{n}\right)-L_{n}\left(T_{n}\right)-n \frac{\partial L_{n}}{\partial n}\left(T_{n}\right)}{C_{p, v}-\frac{R_{v}}{2}}}\right.}{\frac{1-\alpha_{n}}{\alpha_{n}}+\sum_{k=1}^{N_{c}} \frac{\zeta_{n}^{c, k}}{\zeta_{n}^{v}} \frac{\rho_{c, k}}{\rho_{v}} \frac{C_{p, k}-\frac{R_{k}}{2}}{C_{p, v}-\frac{R_{v}}{2}}+\frac{\zeta_{n-1}^{v}}{\zeta_{n}^{v}} S_{n}\left(T_{n}\right) \sqrt{\frac{T_{n}}{T}}} .
$$

The term $S_{n}$ is defined by:

$$
S_{n} \equiv S\left(T_{n}, \rho_{v}\right)=\frac{\rho_{v}}{\rho_{v, n}^{s}\left(T_{n}\right)},
$$

and represents the effective saturation ratio at the curved surface of a droplet of size $n$ and temperature $T_{n}$. It is noted that Eq. (2.52) is only valid in the free molecular regime; for a discussion on more generally applicable models, reference can be made to, e.g., [58].

Figure 2.2. $\mathrm{a}$ and $\mathrm{b}$ give an impression of the relative difference between the wet bulb temperature and the gas temperature for a $\mathrm{D}_{2} \mathrm{O}$ droplet residing in a mixture of $\mathrm{D}_{2} \mathrm{O}$-vapor and inert $\mathrm{N}_{2}$-gas. These plots have been obtained by iteratively solving Eq. (2.52), with $\alpha_{n}=1$, and $L_{n}=L_{b}$, using the material properties given in appendix A. It is clear from Figure 2.2.a that increased supersaturation leads to larger deviations between the gas and droplet temperatures. This is also what is expected: higher supersaturation leads to increased nett condensation, and thus also to a higher rate of latent heat release, which in turn requires higher droplet temperatures to remove this heat from the droplet.

It is noted that the difference between $T_{n}$ and $T$ becomes size-invariant for sufficiently large droplets, which can be observed from Eq. (2.52). In the limit of large $n$ :

$$
\begin{aligned}
\alpha_{n} & \rightarrow 1 \\
\frac{\zeta_{n-1}^{v}}{\zeta_{n}^{v}} & \rightarrow 1 \\
\frac{\zeta_{n}^{c, k}}{\zeta_{n}^{v}} & \rightarrow \sqrt{\frac{m_{1}}{m_{c, k}}},
\end{aligned}
$$


and:

$$
L_{n} \rightarrow L_{b} \Longrightarrow \partial L_{n} / \partial n \rightarrow 0
$$

Therefore, the right-hand side of Eq. (2.52) becomes independent of size for large droplets, which leads to:

$$
T_{n} \rightarrow T_{w},
$$

where $T_{w}$ is the wet bulb temperature for condensation at a flat liquid surface.

In Figure 2.2.b, the influence of the carrier gas density on the the wet bulb temperature $T_{n}$ is shown for various droplet sizes. As one would expect, the wet bulb temperature approaches the gas temperature when $\rho_{c} / \rho_{v} \gg 1$. For the specific case corresponding with Figure 2.2.b, this would require a rather high density ratio of $\rho_{c} / \rho_{v} \sim 10^{3}$.

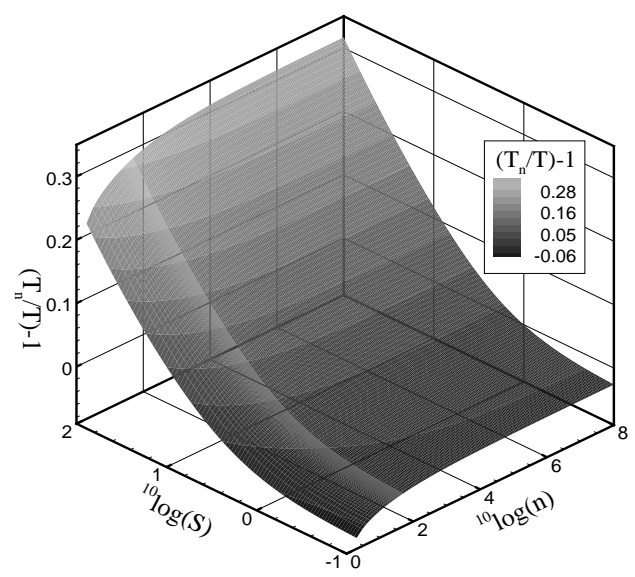

(a)

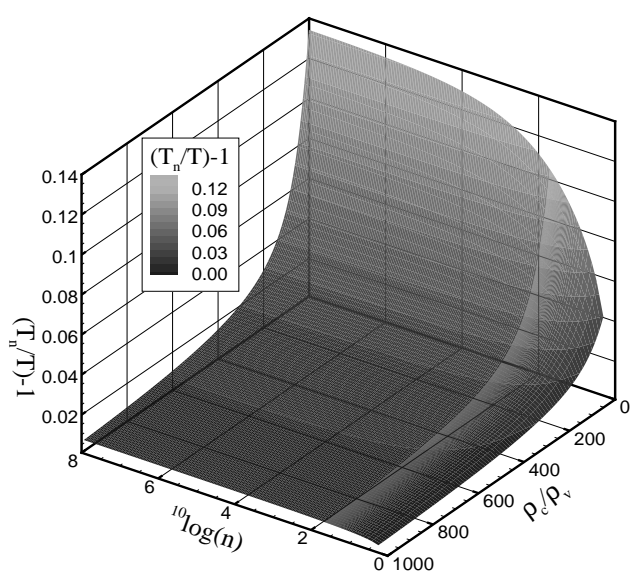

(b)

FIGURE 2.2: Relative difference between droplet-and gas temperature for condensing $\mathrm{D}_{2} \mathrm{O}$, with $\mathrm{N}_{2}$ as the carrier gas: (a) influence of vapor saturation $S$ for various droplet sizes $n\left(T=280 \mathrm{~K}, \rho_{c}=1.0 \mathrm{~kg} / \mathrm{m}^{3}\right) ;(b)$ influence of density ratio $\rho_{c} / \rho_{v}$ for various droplet sizes $n(T=280 K, S=10)$.

In case the thermal relaxation time $\tau_{T}$ is much smaller than the typical time scales associated with variations in the flow field, then Eq. (2.50) shows that the approximation $T^{\prime}(t)=T_{n}(t)$ is allowed. By setting $T^{\prime}=T_{n}\left(\rho_{c, k}, \rho_{v}, T\right)$, the droplet temperature is no longer an independent variable. This is similar to using the algebraic slip model to approximate the droplet velocity. As a consequence, $T^{\prime}$ can also be removed from the list of independent variables associated with the DPD, leaving only the droplet size $n$ as the characterizing variable. Therefore, the bi-variate DPD $\Lambda_{n}^{\prime}\left(T^{\prime}, \mathbf{x}, t\right)$ can 
be replaced with the number density $\hat{\rho}_{n}(\mathbf{x}, t)$, where:

$$
\hat{\rho}_{n}(\mathbf{x}, t)=\int_{\mathbb{R}^{+}} \Lambda_{n}^{\prime}\left(T^{\prime}, \mathbf{x}, t\right) d T^{\prime} .
$$

In the remainder of this thesis, the wet-bulb approximation is employed, unless specified otherwise.

\subsubsection{Mixture thermodynamics}

\section{Non-isothermal condensation}

For moderate pressures, the vapor and inert carrier gas can be treated as perfect gases, and therefore, the corresponding equations of state are given by:

$$
p_{i}=\rho_{i} R_{i} T_{i}
$$

and:

$$
e_{i}=C_{v, i} T_{i},
$$

where the subscript $i$ will be replaced by $c$ for the carrier gas and by $v$ for the vapor. In Eq. (2.54) and Eq. (2.55), $C_{v, i}, \rho_{i}$, and $p_{i}$ denote the constant isochoric specific heat, partial mass density, and partial pressure of component $i$, respectively. In non-isothermal condensation, the droplet temperature may generally differ from the gas/vapor temperature, but the gas phase is assumed to be well-mixed, so that $T_{i}=T$ for all gas constituents.

The amount of liquid within the mixture is given by the liquid mass fraction $g$, which is defined as the ratio of the liquid mass $m_{l}$ to that of the total mixture:

$$
g \equiv \frac{m_{l}}{m_{l}+m_{v}+m_{c}} .
$$

The liquid mass fraction can be calculated from the discrete size distribution $\hat{\rho}_{n}$ by means of the following expression:

$$
g=\frac{m_{1}}{\rho} \sum_{n=2}^{N} n \hat{\rho}_{n},
$$

where the smallest cluster is the dimer $(n=2)$, and the largest is denoted by $N$. Although there is actually no bound on the maximum droplet size $(N \rightarrow \infty$ has been used in Eq. (2.3)), here a finite value for $N$ is adopted for the sake of convenience. For the condensing nozzle flows considered in this thesis, the droplet number density $\hat{\rho}_{n} \approx 0$ for $n>10^{8}$, which means that the maximum droplet size can be set to $N=10^{8}$. 
The total mass fraction of the condensable component in the mixture (i.e., in both liquid and vapor form) is denoted by $g_{\max }$ :

$$
g_{\max }=\frac{m_{l}+m_{v}}{m_{l}+m_{v}+m_{c}} .
$$

As droplet slip is excluded, and diffusion of vapor is neglected, the mixture composition is invariant along fluid trajectories, and therefore, the material derivative of $g_{\max }$ is zero:

$$
\frac{D}{D t}\left(g_{\text {max }}\right)=0 \text {. }
$$

As a consequence, $g_{\max }$ is a global constant in the flow domain, when it is uniformly specified at the inflow boundaries. When the local liquid mass fraction $g$, and mixture density $\rho$ are known, the partial density of each gaseous constituent may be calculated by:

$$
\begin{gathered}
\rho_{c}=\frac{\left(1-g_{\max }\right) \rho}{1-g \frac{\rho}{\rho_{b}}} \\
\rho_{v}=\frac{\left(g_{\max }-g\right) \rho}{1-g \frac{\rho}{\rho_{b}}} .
\end{gathered}
$$

As $\rho / \rho_{b} \sim 10^{-3}$, and because $0 \leq g \leq g_{\max }<1$, the preceding equations may be approximated by:

$$
\begin{aligned}
& \rho_{c}=\left(1-g_{\text {max }}\right) \rho, \\
& \rho_{v}=\left(g_{\text {max }}-g\right) \rho .
\end{aligned}
$$

The mean density $\rho_{l}$ of the liquid dispersion thus follows from:

$$
\rho_{l}=g \rho .
$$

Furthermore, the partial densities $\rho_{c, k}$ of the individual carrier gas components can be calculated from $\rho_{c}$ via the expression:

$$
\rho_{c, k}=w_{c, k} \rho_{c},
$$

where $w_{c, k}$ is the fixed mass fraction of component $k$ with respect to the carrier gas $\left(\sum_{k=1}^{N_{c}} w_{c, k}=1\right)$.

Neglecting the contribution of the liquid dispersion to the pressure, and assuming that the mixture is ideal, the pressure within the mixture is given by:

$$
p=\rho R T
$$


where the specific gas constant $R=R(g)$ for the mixture is given by:

$$
R=\left(1-g_{\max }\right) R_{c}+\left(g_{\max }-g\right) R_{v} .
$$

The total internal energy $e$ per unit mass of the mixture is equal to the sum of the contribution of each of the constituent phases:

$$
e=\left(1-g_{\max }\right) e_{c}(T)+\left(g_{\max }-g\right) e_{v}(T)+\sum_{n=2}^{N} \frac{\hat{\rho}_{n}}{\rho} E_{n},
$$

where $E_{n}$ is the droplet internal energy given by Eq. (2.40). The latter can be rewritten as:

$$
E_{n}=n m_{1}\left[h_{v}^{s}\left(T_{n}\right)-\frac{p}{\rho_{b}\left(T_{n}\right)}-L_{n}\left(T_{n}\right)\right]
$$

Using this expression and the caloric equation of state Eq. (2.55) for the gaseous constituents, Eq. (2.68) can be replaced by:

$$
e=C_{v, 0} T-g C_{v, v} T+m_{1} \sum_{n=2}^{N} \frac{\hat{\rho}_{n}}{\rho} n\left[h_{v}^{s}\left(T_{n}\right)-\frac{p}{\rho_{b}\left(T_{n}\right)}-L_{n}\left(T_{n}\right)\right],
$$

where $C_{v, 0}$ is the isochoric specific heat for the dry mixture,

$$
C_{v, 0} \equiv\left(1-g_{\max }\right) C_{v, c}+g_{\max } C_{v, v}
$$

Due to the large value of the liquid density, the term $p / \rho_{b}$ on the right-hand side of Eq. (2.70) is usually negligibly small compared to the remaining terms. By using a perfect gas model, the vapor enthalpy is reduced to a function of temperature only, so that: $h_{v}^{s}\left(T_{n}\right) \approx h_{v}\left(T_{n}\right)=C_{p, v} T_{n}$. By applying these approximations, the specific mixture energy can be expressed as:

$$
e=C_{v, 0} T-g C_{v, v} T+m_{1} \sum_{n=2}^{N} \frac{\hat{\rho}_{n}}{\rho} n\left[C_{p, v} T_{n}-L_{n}\left(T_{n}\right)\right],
$$

and correspondingly, the mixture specific enthalpy follows from:

$$
h=C_{p, 0} T-g C_{p, v} T+m_{1} \sum_{n=2}^{N} \frac{\hat{\rho}_{n}}{\rho} n\left[C_{p, v} T_{n}-L_{n}\left(T_{n}\right)\right],
$$

where:

$$
C_{p, 0} \equiv\left(1-g_{\max }\right) C_{p, c}+g_{\max } C_{p, v} .
$$

By using the wet-bulb approximation for the droplet temperature, the specific internal energy of the mixture becomes dependent on the droplet size distribution $\hat{\rho}_{n}$, the 
partial mass densities of the vapor and carrier gas components $\left(\rho_{v}\right.$, and $\rho_{c, k}$, respectively), and the gas temperature $T$. Since $\rho_{v}$ and $\rho_{c, k}$ are determined from the mixture mass density $\rho$ and the number densities $\hat{\rho}_{n}$ by means of Eqs. (2.57), (2.62), (2.63), and (2.65), the specific internal energy of the mixture is reduced to a function of the mixture density, the gas temperature and the droplet size distribution: $e=e(\rho, \hat{\rho}, T)$, with $\hat{\boldsymbol{\rho}}=\left(\hat{\rho}_{2}, \hat{\rho}_{3}, \ldots, \hat{\rho}_{N}\right)^{T}$. The caloric equation of state for the mixture is thus rather complex, even though the perfect gas model has been adopted for the gaseous phase. It is noted that from $e=e(\rho, \hat{\boldsymbol{\rho}}, T)$, it also follows that $T=T(\rho, \hat{\boldsymbol{\rho}}, e)$. Due to Eqs. (2.57), (2.66), and (2.67), the pressure assumes the same dependency: $p=p(\rho, \hat{\boldsymbol{\rho}}, e)$.

\section{Isothermal condensation}

Isothermal condensation refers to equality of the droplet and gas temperatures: $T_{n}=$ $T$, and should not be confused with the usual thermodynamic definition of timeinvariant temperature. Because the mixture is characterized by a single temperature, Eq. (2.72) for the specific internal energy of the mixture can be simplified to:

$$
e=C_{v, 0} T+g R_{v} T-m_{1} \sum_{n=2}^{N} \frac{\hat{\rho}_{n}}{\rho} n L_{n}(T),
$$

and correspondingly, the specific mixture enthalpy becomes:

$$
h=C_{p, 0} T-m_{1} \sum_{n=2}^{N} \frac{\hat{\rho}_{n}}{\rho} n L_{n}(T) .
$$

For large droplets, $L_{n} \rightarrow L_{b}$, which implies that for a dispersion which predominantly consists of such droplets, the preceding equations can be approximated by:

$$
e=C_{v, 0} T+g\left[R_{v} T-L_{b}(T)\right]
$$

and:

$$
h=C_{p, 0} T-g L_{b}(T) .
$$

As a consequence, the internal energy $e$ now only depends on the liquid mass fraction $g$ and the temperature $T$, i.e., $e=e(T, g)$. Conversely, $T=T(e, g)$, and therefore, it follows from Eqs. (2.66) and (2.67) that: $p=p(\rho, e, g)$.

In the numerical simulation of condensing flow, the caloric equations of state for the mixture (Eq. (2.72), or its iso-thermal variant Eq. (2.77)) are used to determine the temperature from the DSD and the internal energy and density of the mixture. For the case of isothermal condensation, knowledge of $e$ and $g$ is sufficient to (iteratively) solve Eq. (2.77) for $T$. For the non-isothermal case, however, the size-dependency of the wet-bulb temperature complicates matters, as $T_{n}$ itself depends on $T$. Since $T_{n}$ is 
iteratively solved from Eq. (2.52), the calculation of $T$ from $e, \rho$, and $\hat{\rho}_{n}$ is a rather expensive operation, which, if possible, should be avoided in numerical simulations. In this regard, it is instructive to examine the relative difference $\Delta e$ :

$$
\Delta e=\frac{e_{i s o}-e_{n-i s o}}{e_{n-i s o}}
$$

where $e_{n-i s o}$, and $e_{i s o}$ are the internal energies obtained by means of Eqs. (2.72) and (2.77), respectively. To simplify matters, the size-dependency of the latent heat of condensation and the wet-bulb temperature are neglected, i.e., $L_{n}=L_{b}$, and: $T_{n}=T_{w}$, so that Eq. (2.72) for the specific internal energy can be simplified to:

$$
e=C_{v, 0} T-g C_{v, v} T+g\left[C_{p, v} T_{w}-L_{b}\left(T_{w}\right)\right] .
$$

Using Eq. (2.79), it suffices to know only the liquid mass fraction $g$, instead of the complete DSD $\hat{\rho}_{n}$, to compute the specific internal energy $e$. The assumption $T_{n}=$ $T_{w}$ can be justified by noting that the largest droplets show the largest differences between $T_{n}$ and $T$ (see Figures 2.2.a and b), so that by setting $T_{n}=T_{w}$, the relative difference $\Delta e$ is overestimated. Figure 2.3.a shows how $\Delta e$ varies with $g$ and $T$, for a case of condensing $\mathrm{D}_{2} \mathrm{O}$ in a mixture of $\mathrm{D}_{2} \mathrm{O}$ and inert $\mathrm{N}_{2}$-gas, with $g_{\max }=0.018$ and $\rho_{c}=1.0 \mathrm{~kg} / \mathrm{m}^{3}$. It is noted that although the differences between $T_{w}$ and $T$ are $\sim 10^{-1}$ (see Figure 2.3.b), the relative difference $\Delta e$ is $\sim 10^{-3}-10^{-2}$. Of course, these small differences are a consequence of the relatively low vapor mass fraction $g_{\text {max }}$ used. For higher values of $g_{\max }$, the contribution of the liquid phase to the internal energy can be potentially higher, which means that one is compelled to use Eq. (2.72) for such cases.

\subsubsection{Mixture transport equations}

\section{Conservation form}

The conservation equations for mass, momentum and energy for the general case of slipping droplets are extensively discussed by Young in [149], and therefore, only the special case of non-slipping droplets will be discussed here. In differential form, the continuity equations for the carrier gas, vapor, and droplet size distribution are given by:

$$
\begin{gathered}
\frac{\partial \rho_{c}}{\partial t}+\frac{\partial}{\partial x_{j}}\left(\rho_{c} u_{j}\right)=0, \\
\frac{\partial \rho_{v}}{\partial t}+\frac{\partial}{\partial x_{j}}\left(\rho_{v} u_{j}\right)=-m_{1} \sum_{n=2}^{N} n \tilde{S}_{n}, \\
\frac{\partial \hat{\rho}_{n}}{\partial t}+\frac{\partial}{\partial x_{j}}\left(\hat{\rho}_{n} u_{j}\right)=\tilde{S}_{n}, \quad, n=2,3, . ., N-1,
\end{gathered}
$$




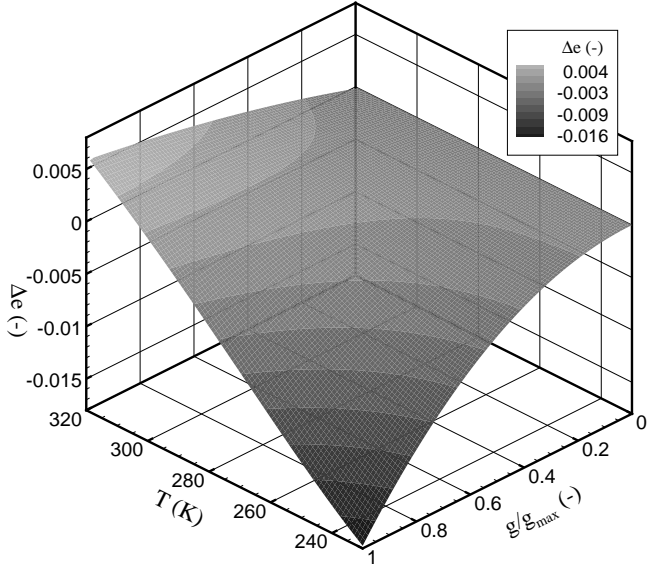

(a)

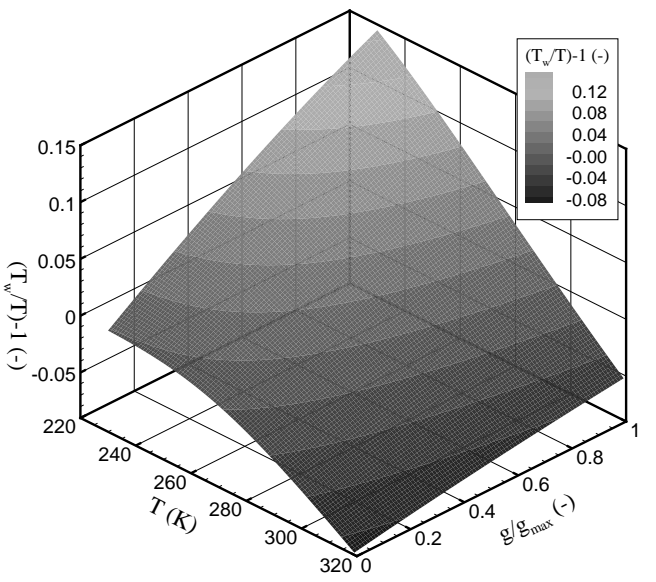

(b)

Figure 2.3: (a) Relative difference between $e_{\text {iso }}$ (Eq. (2.77)) and $e_{n-s i o}(E q .(2.72))$ for a mixture of condensing $\mathrm{D}_{2} \mathrm{O}$, with $\mathrm{N}_{2}$ as the carrier gas $\left(g_{\max }=0.018, \rho_{c}=\right.$ $\left.1.0 \mathrm{~kg} / \mathrm{m}^{3}\right)$; (b) corresponding relative difference between $T$ and $T_{w}$.

with $\tilde{S}_{n}$ denoting a condensation source term, to be elaborated in chapter 3 . It is noted that the balance equation for the monomers is represented by that of the vapor, and that the largest cluster of size $N$ is omitted from Eq. (2.82), because of the boundary condition $\hat{\rho}_{N}=0$ in $n$-space. With $\rho_{l}=m_{1} \sum_{n=2}^{N} n \hat{\rho}_{n}$, the conservation equation for the liquid mass density is derived as:

$$
\frac{\partial \rho_{l}}{\partial t}+\frac{\partial}{\partial x_{j}}\left(\rho_{l} u_{j}\right)=m_{1} \sum_{n=2}^{N} n \tilde{S}_{n}
$$

Since the mixture density satisfies: $\rho=\rho_{c}+\rho_{v}+\rho_{l}$, the continuity equation for the mixture becomes:

$$
\frac{\partial \rho}{\partial t}+\frac{\partial}{\partial x_{j}}\left(\rho u_{j}\right)=0
$$

as the source terms for the liquid dispersion and the vapor cancel each other.

It is noted that none of the preceding mass balance equations contain the effect of vapor diffusion. The importance of vapor diffusion compared to advection is quantified by the Péclet number Pé, defined by [14]:

$$
P e ́ \equiv \frac{U_{0} L_{0}}{D_{v, c}}
$$

where $D_{v, c}$ is the diffusion coefficient of the vapor in the carrier gas, and where $U_{0}$ and $L_{0}$ are typical velocity and length scales of the flow. For transonic condensing 
flows, $U_{0} \sim 10^{2} \mathrm{~m} / \mathrm{s}$, and $L_{0} \sim 10^{-2}-10^{-1} \mathrm{~m}$ for the nozzles considered here. For $200 \leq T \leq 300 \mathrm{~K}$, and $p \sim 10^{4} \mathrm{~Pa}$, the diffusion coefficient is $D_{v, c} \sim 10^{-3} \mathrm{~m}^{2} \mathrm{~s}^{-1}$. Thus: $P e ́\left(10^{3} \gg 1\right.$, and therefore, diffusion of vapor at the length scales of the flow can be neglected. It is noted that for low Knudsen numbers (i.e., relatively large droplets), the Péclet number associated with the droplet size and its slip velocity is much smaller than unity. Thus, on small scales, diffusion is essential for condensation of droplets when $K n \ll 1$, whereas on large scales, it is of negligible influence.

Neglecting viscous stresses, the momentum equation for the mixture is given by:

$$
\frac{\partial}{\partial t}\left(\rho u_{i}\right)+\frac{\partial}{\partial x_{j}}\left(\rho u_{i} u_{j}+p \delta_{i j}\right)=0
$$

For transonic nozzle flows without flow separation, viscous effects are confined to the boundary layer at the channel wall, where the velocity gradients are the largest. For such conditions, the flow is nearly inviscid away from the boundary layer, as has been observed in experiments [100]. Since viscosity is also not essential for condensation to occur, it is neglected entirely.

Neglecting viscous dissipation and heat conduction, the energy balance for the mixture becomes:

$$
\frac{\partial}{\partial t}\left(\rho e_{t}\right)+\frac{\partial}{\partial x_{j}}\left(\rho h_{t} u_{j}\right)=0,
$$

where $e_{t}$ and $h_{t}$ represent the total energy,

$$
e_{t}=e+\frac{1}{2} u_{j} u_{j}
$$

and total enthalpy,

$$
h_{t}=h+\frac{1}{2} u_{j} u_{j}
$$

of the mixture, respectively. The omission of heat conduction from the model can be justified in a similar way as was derived for the diffusion of vapor. The Péclet number is now defined as:

$$
P e ́ \equiv \frac{U_{0} L_{0}}{a_{T}},
$$

where $a_{T}$ is the thermal diffusion coefficient of the gas phase. Using the same numerical values for $U_{0}$ and $L_{0}$ as before, and noting that $D_{v c}$ and $a_{T}$ are of the same order of magnitude, it is again found that Pé $\gg 1$, which makes it justified to neglect heat diffusion.

\section{Characteristic form}

The characteristic forms of Eq. (2.84), (2.86), and (2.87), augmented by Eq. (2.82), are relevant in case boundary conditions at inflow- or outflow boundaries are applied. 
Although the characteristic equations can be derived for a general three-dimensional flow as demonstrated in [53], here it suffices to treat the one-dimensional case.

In order to derive the characteristic equations, it is more convenient to use the following Lagrangian formulation of Eq. (2.82) for the specific number density $\check{\rho}_{n} \equiv$ $\hat{\rho}_{n} / \rho$ :

$$
\frac{D \check{\rho}_{n}}{D t}=\check{S}_{n}, \quad n=2,3, . . N-1,
$$

where $\breve{S}_{n}=\tilde{S}_{n} / \rho$, and $D / D t$ denotes the material derivative. By using the mass balance, the one-dimensional version of the momentum and energy balance equations can be written in the following non-conservative form:

$$
\begin{aligned}
& \frac{D u}{D t}+\frac{1}{\rho} \frac{\partial p}{\partial x}=0, \\
& \frac{D e}{D t}+\frac{p}{\rho} \frac{\partial u}{\partial x}=0 .
\end{aligned}
$$

Introducing $\mathbf{q}=\left(\rho, u, e, \check{\rho}_{2}, . ., \check{\rho}_{n}, . ., \check{\rho}_{N-1}\right)^{T}$ as the state vector of primitive variables, and noting that $p=p\left(e, \rho, \check{\rho}_{2}, . ., \check{\rho}_{N-1}\right)$, the system of transport equations (encompassing Eq. (2.91)) can be written as:

$$
\frac{\partial \mathbf{q}}{\partial t}+\mathbf{J}_{q} \frac{\partial \mathbf{q}}{\partial x}=\check{\mathbf{S}}
$$

where the Jacobi-matrix $\mathbf{J}_{q}$ is given by:

$$
\mathbf{J}_{q}=\left(\begin{array}{cccccccc}
u & \rho & 0 & 0 & . . & 0 & . . & 0 \\
\frac{1}{\rho} \frac{\partial p}{\partial \rho} & u & \frac{1}{\rho} \frac{\partial p}{\partial e} & \frac{1}{\rho} \frac{\partial p}{\partial \check{\rho}_{2}} & . . & \frac{1}{\rho} \frac{\partial p}{\partial \check{\rho}_{n}} & . . & \frac{1}{\rho} \frac{\partial p}{\partial \check{\rho}_{N-1}} \\
0 & \frac{p}{\rho} & u & 0 & . . & 0 & . . & 0 \\
0 & 0 & 0 & u & . . & 0 & . . & 0 \\
: & & & & & & & : \\
0 & 0 & 0 & 0 & . . & u & . . & 0 \\
: & & & & & & & : \\
0 & 0 & 0 & 0 & . . & 0 & . . & u
\end{array}\right),
$$

and the source vector $\check{\mathbf{S}}$ by:

$$
\check{\mathbf{S}}=\left(0,0,0, \check{S}_{2}, \check{S}_{3}, . ., \check{S}_{N-1}\right)^{T}
$$

The eigenvalues $\lambda_{k}$ of $\mathbf{J}_{q}$ are obtained by solving $\operatorname{Det}\left|\mathbf{J}_{q}-\lambda_{k} \mathbf{I}\right|=0$, where $\mathbf{I}$ is the identity matrix. Expanding this equation leads to the following polynomial expression:

$$
\left(u-\lambda_{k}\right)^{N-1}\left[\left(u-\lambda_{k}\right)^{2}-\left\{\frac{\partial p}{\partial \rho}+\frac{p}{\rho^{2}} \frac{\partial p}{\partial e}\right\}\right]=0 .
$$


It is noted that even though the dependence of $p$ on $\check{\rho}_{n}$ has been taken into account, the characteristic polynomial does not contain any of the derivatives $\frac{\partial p}{\partial \check{\rho}_{n}}$. The term within the curly brackets is recognized as the square of the frozen speed of sound $a_{f}$ :

$$
\left(a_{f}\right)^{2}=\left(\frac{\partial p}{\partial \rho}\right)_{e, g}+\frac{p}{\rho^{2}}\left(\frac{\partial p}{\partial e}\right)_{\rho, g} .
$$

Using Eq. (2.98), solution of Eq. (2.97) yields the following set of eigenvalues:

$$
\lambda_{1}=u ; \lambda_{2}=u+a_{f} ; \lambda_{3}=u-a_{f} ; \lambda_{k}=u, \text { for } k=4,5, . ., N+1 .
$$

Having determined the eigenvalues, the next step is to derive the corresponding left eigenvectors $\mathbf{L}_{k}$, which follow from:

$$
\mathbf{L}_{k}^{T}\left(\mathbf{J}_{q}-\lambda_{k} \mathbf{I}\right)=0 .
$$

Solving for $\mathbf{L}_{k}$ then yields the first three eigenvectors:

$$
\begin{aligned}
& \mathbf{L}_{1}=\left(-\frac{p}{\rho^{2}}, 0,1,0, \ldots, 0, . ., 0\right)^{T}, \\
& \mathbf{L}_{2}=\left(\frac{1}{\rho a_{f}} \frac{\partial p}{\partial \rho}, 1, \frac{1}{\rho a_{f}} \frac{\partial p}{\partial e}, \frac{1}{\rho a_{f}} \frac{\partial p}{\partial \check{\rho}_{2}}, . ., \frac{1}{\rho a_{f}} \frac{\partial p}{\partial \check{\rho}_{n}}, . ., \frac{1}{\rho a_{f}} \frac{\partial p}{\partial \check{\rho}_{N-1}}\right)^{T}, \\
& \mathbf{L}_{3}=\left(-\frac{1}{\rho a_{f}} \frac{\partial p}{\partial \rho}, 1,-\frac{1}{\rho a_{f}} \frac{\partial p}{\partial e},-\frac{1}{\rho a_{f}} \frac{\partial p}{\partial \check{\rho}_{2}}, . .,-\frac{1}{\rho a_{f}} \frac{\partial p}{\partial \check{\rho}_{n}}, . .,-\frac{1}{\rho a_{f}} \frac{\partial p}{\partial \check{\rho}_{N-1}}\right)^{T},
\end{aligned}
$$

whereas for the remaining eigenvectors $(k \geq 4)$, the components are given by:

$$
L_{k n}=\delta_{k n},
$$

with $\delta_{k n}$ denoting the Kronecker delta.

In order to obtain the characteristic form of the transport equations, Eq. (2.94) is multiplied with the left eigenvectors. Subsequent manipulations using the relationship:

$$
d p=\frac{\partial p}{\partial \rho} d \rho+\frac{\partial p}{\partial e} d e+\frac{\partial p}{\partial \check{\rho}_{j}} d \check{\rho}_{j},
$$

in combination with with Eq. (2.98), finally yields the first three characteristic equations:

$$
\begin{aligned}
\frac{\partial \rho}{\partial t}-\frac{1}{a_{f}^{2}} \frac{\partial p}{\partial t}+u\left(\frac{\partial \rho}{\partial x}-\frac{1}{a_{f}^{2}} \frac{\partial p}{\partial x}\right) & =-\frac{1}{a_{f}^{2}} \frac{\partial p}{\partial \check{\rho}_{j}} \breve{S}_{j} \\
\frac{\partial u}{\partial t}+\frac{1}{\rho a_{f}} \frac{\partial p}{\partial t}+\left(u+a_{f}\right)\left(\frac{\partial u}{\partial x}+\frac{1}{\rho a_{f}} \frac{\partial p}{\partial x}\right) & =\frac{1}{\rho a_{f}} \frac{\partial p}{\partial \check{\rho}_{j}} \breve{S}_{j} \\
\frac{\partial u}{\partial t}-\frac{1}{\rho a_{f}} \frac{\partial p}{\partial t}+\left(u-a_{f}\right)\left(\frac{\partial u}{\partial x}-\frac{1}{\rho a_{f}} \frac{\partial p}{\partial x}\right) & =-\frac{1}{\rho a_{f}} \frac{\partial p}{\partial \check{\rho}_{j}} \breve{S}_{j},
\end{aligned}
$$


whereas the remaining component equations are identical to Eq. (2.91). It is noted that in contrast to the conservative form of the FDE, viz. Eqs. (2.84), (2.86), and (2.87), all of the characteristic equations do contain source term contributions arising from condensation.

The characteristic equations are essential in specifying the appropriate boundary conditions at in- or outflow boundaries. In one dimension, there are essentially three characteristics, along which information travels through physical space with the velocities $u$, and $u \pm a_{f}$. For subsonic inflow, two characteristics with speed $u$ and $u+a_{f}$ correspond to information entering the flow domain from the boundary, whereas the characteristic with speed $u-a_{f}$ corresponds to information leaving the flow domain. As a consequence, only the size distribution and two flow variables can be specified at subsonic inflow boundaries. For supersonic inflow, all characteristics correspond to information entering the flow domain. As a consequence, all flow variables can be specified at this boundary, along with the size distribution. For subsonic outflow, two characteristics correspond to information leaving the flow domain, whereas one characteristic corresponds to information entering the flow domain. As a consequence, one of the flow variables (usually the pressure) needs to be specified at the outlet. In the case of supersonic outflow, all characteristics correspond to information leaving the flow domain, meaning that none of the variables needs to be specified at this boundary.

\section{Speed of sound}

Analysis of the eigenvalues of the Jacobi-matrix $\mathbf{J}_{q}$ has revealed that the appropriate speed of sound to be used in condensing flow is the frozen speed of sound $a_{f}$, given by Eq. (2.98). To actually calculate $a_{f}$, it is necessary to evaluate the partial derivatives of $p$ with respect to $\rho$ and $e$. For the non-isothermal condensation model, the relevant expressions needed to compute these derivatives are Eqs. (2.52), (2.57), (2.66), (2.67), and (2.72). Noting that $g=g(\check{\boldsymbol{\rho}})$, and $T=T(e, \rho, \check{\boldsymbol{\rho}})$, with: $\check{\boldsymbol{\rho}}=\left(\check{\rho}_{2}, \check{\rho}_{3}, . .\right)^{T}$, the equation for the pressure can be written as:

$$
p=p(e, \rho, \check{\rho})=\rho R(\check{\rho}) T(e, \rho, \check{\rho}) .
$$

It is thus straightforward to write:

$$
\frac{\partial p}{\partial \rho}=R\left[T+\rho \frac{\partial T}{\partial \rho}\right]
$$

and:

$$
\frac{\partial p}{\partial e}=\rho R \frac{\partial T}{\partial e}
$$

so that the problem is now reduced to finding the partial derivatives of $T$ with respect to $\rho$ and $e$. The temperature is implicitly given by Eqs. (2.72) and (2.52). To determine $\partial T / \partial \rho$ and $\partial T / \partial e$, it is first necessary to determine the dependency of the wet 
bulb temperature $T_{n}$ on $\rho$ and $e$. The dependency on $e$ is only due to the occurrence of $T=T(e, \rho, \check{\rho})$ in Eq. (2.52). The dependency on $\rho$ is due to $T=T(e, \rho, \check{\rho})$, and due to the saturation ratio $S_{n}$, since from:

$$
S_{n}=S_{n}\left(T_{n}, \rho_{v}\right)=\frac{\rho_{v}}{\rho_{v, n}^{s}\left(T_{n}\right)}=\frac{\rho\left(g_{\max }-g(\check{\rho})\right)}{\rho_{v, n}^{s}\left(T_{n}\right)}
$$

it follows that $S_{n}=S_{n}\left(T_{n}, \rho, \check{\boldsymbol{\rho}}\right)$. It is noted that the density ratio's $\rho_{c, k} / \rho_{v}$ can be expressed as

$$
\frac{\rho_{c, k}}{\rho_{v}}=w_{c, k} \frac{1-g_{\max }}{g_{\max }-g(\check{\boldsymbol{\rho}})},
$$

which makes them independent of $\rho$. In conclusion, the dependency of $T_{n}$ can be expressed as: $T_{n}=T_{n}(\rho, \check{\boldsymbol{\rho}}, T(e, \rho, \check{\boldsymbol{\rho}}))$. Using this dependency, differentiation of Eq. (2.72) with respect to $e$, followed by some further manipulations, yields:

$$
\frac{\partial T}{\partial e}=\left\{C_{v, 0}-g C_{v, v}+m_{1} \sum_{n=2}^{N} \check{\rho}_{n} n\left(C_{p, v}-\frac{\partial L_{n}}{\partial T_{n}}\right)\left(\frac{\partial T_{n}}{\partial T}\right)_{\rho, \check{\rho}}\right\}^{-1} .
$$

Taking the derivative of Eq. (2.72) with respect to $\rho$, with some subsequent manipulations, yields:

$$
\frac{\partial T}{\partial \rho}=-\frac{m_{1} \sum_{n=2}^{N} \check{\rho}_{n} n\left(C_{p v}-\frac{\partial L_{n}}{\partial T_{n}}\right)\left(\frac{\partial T_{n}}{\partial \rho}\right)_{T, \check{\rho}}}{C_{v, 0}-g C_{v, v}+m_{1} \sum_{n=2}^{N} \check{\rho}_{n} n\left(C_{p, v}-\frac{\partial L_{n}}{\partial T_{n}}\right)\left(\frac{\partial T_{n}}{\partial T}\right)_{\rho, \check{\boldsymbol{\rho}}}} .
$$

What remains to be determined now are the partial derivatives:

$$
\left(\frac{\partial T_{n}}{\partial \rho}\right)_{T, \check{\rho}} \text {, and }\left(\frac{\partial T_{n}}{\partial T}\right)_{\rho, \check{\boldsymbol{\rho}}},
$$

which can be obtained by first solving for $T_{n}(\rho, \check{\rho}, T)$ for given $\rho, \check{\rho}$, and $T$, followed by numerical differentiation with respect to $\rho$ and $T$, respectively.

When the isothermal condensation model is used, the previous expressions are greatly simplified. Since: $T_{n} \rightarrow T$, it follows immediately that:

$$
\left(\frac{\partial T_{n}}{\partial \rho}\right)_{T, \check{\rho}}=0,\left(\frac{\partial T_{n}}{\partial T}\right)_{\rho, \check{\rho}}=\frac{d T}{d T}=1,
$$

which subsequently leads to:

$$
\frac{\partial T}{\partial e}=\left\{C_{v, 0}-g C_{v, v}+m_{1} \sum_{n=2}^{N} \check{\rho}_{n} n\left(C_{p, v}-\frac{\partial L_{n}}{\partial T}\right)\right\}^{-1},
$$


and:

$$
\frac{\partial T}{\partial \rho}=0
$$

The frozen speed of sound thus becomes:

$$
\left(a_{f}\right)^{2}=R T\left[1+\frac{R}{C_{v, 0}-g C_{v, v}+m_{1} \sum_{n=2}^{N} \check{\rho}_{n} n\left(C_{p, v}-\frac{\partial L_{n}}{\partial T}\right)}\right] .
$$

If the dispersion is dominated by large droplets, then $L_{n}$ can be replaced by $L_{b}$, so that one finally obtains:

$$
\left(a_{f}\right)^{2}=R T\left[1+\frac{R}{C_{v, 0}+g\left(R_{v}-\frac{d L_{b}}{d T}\right)}\right] .
$$

\subsection{Multi-component condensation}

\subsubsection{Description of constituent phases}

In multi-component condensation, the gas phase generally consists of a mixture of different condensing vapors and several inert gases, and the droplets that make up the dispersion consist of the various chemical species corresponding with the vapors. Following the same line of discussion as in the case of single-component condensation, the liquid dispersion can again be characterized by a multi-variate droplet property distribution function $\Lambda_{\mathbf{n}}\left(T^{\prime}, \mathbf{v}, \mathbf{x}, t\right)$, where $\mathbf{n}$ is the vector $\left(n_{1}, n_{2}, . ., n_{N_{v}}\right)^{T}$. Here, $n_{j}$ represents the number of molecules of component $j$ contained in a droplet, and $N_{v}$ denotes the total number of condensing components. Clearly, $n_{j} \in \mathbb{N}$, and the smallest of droplets are either unary, or binary dimers. The multi-component droplet number densities $\hat{\rho}_{\mathbf{n}}$ can be calculated from $\Lambda_{\mathbf{n}}(\mathbf{v}, T, \mathbf{x}, t)$ by means of the expression:

$$
\hat{\rho}_{\mathbf{n}}(\mathbf{x}, t)=\int_{\mathbb{R}^{+}} \int_{\mathbb{R}^{3}} \Lambda_{\mathbf{n}}\left(T^{\prime}, \mathbf{v}, \mathbf{x}, t\right) d \mathbf{v} d T^{\prime} .
$$

The use of macroscopic models in multi-component condensation suffers from the same deficiencies as already discussed for single-component condensation, and will not be repeated here. In principle, one could maintain the full dependency of the multi-component DPD, which means that in total, there are $8 N^{N_{v}}$ independent variables that need to be dealt with in the general case of a transient, and threedimensional flow ( $N$ is the maximum value of $n_{j}$ ). By using an algebraic slip model 
and the wet-bulb approximation, the droplet velocity and temperature can be dropped from the list of independent variables, so that $\Lambda_{\mathbf{n}}\left(T^{\prime}, \mathbf{v}, \mathbf{x}, t\right)$ can be replaced with the multi-component droplet number densities $\hat{\rho}_{\mathbf{n}}$. As a consequence, the number of independent variables reduces to $4 N^{N_{v}}$, which still poses a computationally challenging problem, even when considering the case of binary condensation $\left(N_{v}=2\right)$. The solution of the multi-component DPD has therefore only been attempted for the binary case in stagnant systems, such as in [74] and [142].

It is noted that even when using the full dependency in the formulation of the DPD, there are still a number of important factors which are excluded. One of these is the spatial distribution of the various components within the interior of the droplets and at the droplet surface. The latter determines the value of the surface tension, which, in turn, has a pronounced influence on the condensation rate. Usually, the liquid within a droplet is assumed to be well-mixed, if the liquid components are mutually miscible. For immiscible components, however, a layered droplet model may be adopted, which means that the transport of condensing components through the liquid layers needs to be included in the model [85]. The multi-component condensation model presented in this thesis assumes a uniform spatial distribution of the liquid components, which implies that only condensation of well-mixed components is considered. To avoid using an overcomplicated model with many associated physical uncertainties, it is furthermore assumed that the droplets are uniform in temperature, with $T^{\prime}=T$ (i.e., isothermal multi-component condensation is assumed).

\subsubsection{Definition of mass densities and compositions}

Before embarking on the thermodynamics of the constituent phases, it is first necessary to define a number of variables related to the composition of the mixture. The indexation of variables is as follows: ' $c, k$ ' refers to the $k^{\text {th }}$-component of the carrier gas, ' $v, k$ ' refers to the $k^{\text {th }}$-component of the vapor phase, 'n, $k$ ' refers to the $k^{\text {th }}$ component in a droplet of composition $\mathbf{n}=\left(n_{1}, n_{2}, . ., n_{N_{v}}\right)^{T}$, and ' $l, k$ ' refers to the $k^{\text {th }}$-component in the liquid phase (i.e., the liquid dispersion). The vapor components share the same second indices with the individual droplets and the liquid phase, i.e., ' $v, k$ ', 'n, $k$ ', and ' $l, k$ ' all refer to the same substance, but only in different phases. $N_{v}$ and $N_{c}$ denote the number of vapor and carrier gas components of the mixture, respectively.

\section{Droplet and monomer masses}

Using these conventions, the monomer masses of vapor component $k$, and carrier gas component $k$ are denoted by $m_{v, k}$ and $m_{c, k}$, respectively. The mass of vapor component $k$ in an $\mathbf{n}$-droplet is denoted by $m_{\mathbf{n}, k}$, and satisfies:

$$
m_{\mathbf{n}, k}=n_{k} m_{v, k}
$$


whereas the total mass $m_{\mathbf{n}}$ of an $\mathbf{n}$-droplet is equal to:

$$
m_{\mathbf{n}}=\sum_{k=1}^{N_{v}} m_{\mathbf{n}, k}
$$

\section{Component mass densities}

The mass densities of component $k$ in the vapor phase, component $k$ in the carrier gas, and component $k$ of $\mathbf{n}$-droplets are denoted by $\rho_{v, k}, \rho_{c, k}$, and $\rho_{\mathbf{n}, k}$, respectively. The latter can be calculated from the droplet number density $\hat{\rho}_{\mathbf{n}}$ and the droplet component mass $m_{\mathbf{n}, k}$, via the expression:

$$
\rho_{\mathbf{n}, k}=\hat{\rho}_{\mathbf{n}} m_{\mathbf{n}, k} .
$$

Knowing $\rho_{\mathbf{n}, k}$, the mass density $\rho_{l, k}$ of component $k$ in the liquid phase then follows from:

$$
\rho_{l, k}=\sum_{\mathbf{n} \in \Omega_{\mathbf{n}}} \rho_{\mathbf{n}, k},
$$

where $\Omega_{\mathbf{n}}$ includes only droplets, i.e.,

$$
\Omega_{\mathbf{n}}=\left\{\mathbf{n} \in \mathbb{N}^{N_{v}} \mid\|\mathbf{n}\|>1\right\} .
$$

\section{Total mass densities}

The total mass densities of the carrier gas, vapor phase, $\mathbf{n}$-droplets, and liquid phase are denoted by $\rho_{c}, \rho_{v}, \rho_{\mathbf{n}}$, and $\rho_{l}$, respectively, and relate as follows to the component mass densities:

$$
\rho_{c}=\sum_{k=1}^{N_{c}} \rho_{c, k}, \rho_{v}=\sum_{k=1}^{N_{v}} \rho_{v, k}, \quad \rho_{\mathbf{n}}=\sum_{k=1}^{N_{v}} \rho_{\mathbf{n}, k}, \quad \rho_{l}=\sum_{k=1}^{N_{v}} \rho_{l, k} .
$$

The total mixture mass density is, evidently, given by:

$$
\rho=\rho_{c}+\rho_{v}+\rho_{l}
$$

\section{Phasic mass fractions}

The phasic mass fractions of component $k$ of the carrier gas, component $k$ of the vapor phase, component $k$ of $\mathbf{n}$-droplets, and component $k$ of the liquid phase are denoted by $w_{c, k}, w_{v, k}, w_{\mathbf{n}, k}$, and $w_{l, k}$, respectively, and can be calculated from the component mass densities and total mass densities as follows:

$$
w_{c, k}=\frac{\rho_{c, k}}{\rho_{c}}, w_{v, k}=\frac{\rho_{v, k}}{\rho_{v}}, w_{\mathbf{n}, k}=\frac{\rho_{\mathbf{n}, k}}{\rho_{\mathbf{n}}}, w_{l, k}=\frac{\rho_{l, k}}{\rho_{l}} .
$$


It is noted that all preceding mass fractions have been normalized with respect to their corresponding phases, i.e.,

$$
\sum_{k=1}^{N_{c}} w_{c, k}=1, \quad \sum_{k=1}^{N_{v}} w_{v, k}=1, \quad \sum_{k=1}^{N_{v}} w_{\mathbf{n}, k}=1, \quad \sum_{k=1}^{N_{v}} w_{l, k}=1 .
$$

\section{Mixture mass fractions}

The mixture mass fractions of component $k$ of the carrier gas, component $k$ of the vapor phase, component $k$ of $\mathbf{n}$-droplets, and component $k$ of the liquid phase are denoted by $g_{c, k}, g_{v, k}, g_{\mathbf{n}, k}$, and $g_{l, k}$, respectively, and relate as follows to the total and component mass densities:

$$
g_{c, k}=\frac{\rho_{c, k}}{\rho}, g_{v, k}=\frac{\rho_{v, k}}{\rho}, g_{\mathbf{n}, k}=\frac{\rho_{\mathbf{n}, k}}{\rho}, g_{l, k}=\frac{\rho_{l, k}}{\rho} .
$$

The total mixture mass fraction of condensable component $k$ (i.e., in both liquid and vapor phase), denoted by $g_{v, k}^{\max }$, and with:

$$
g_{v, k}^{\max }=g_{v, k}+g_{l, k},
$$

is invariant along fluid trajectories when droplet slip and vapor diffusion are neglected.

\section{Phasic molar fractions}

Instead of using the mass fractions, the phasic compositions can also be described in terms of the phasic molar fractions. The phasic molar fractions of component $k$ of the vapor phase, component $k$ of $\mathbf{n}$-droplets, and component $k$ of the liquid phase are denoted by $y_{k}, x_{\mathbf{n}, k}$, and $x_{l, k}$, respectively, and can be calculated from the component mass densities as follows:

$$
y_{k}=\frac{\rho_{v, k} / m_{v, k}}{\sum_{m=1}^{N_{v}} \rho_{v, m} / m_{v, m}}, \quad x_{\mathbf{n}, k}=\frac{\rho_{\mathbf{n}, k} / m_{v, k}}{\sum_{m=1}^{N_{v}} \rho_{\mathbf{n}, m} / m_{v, m}}, x_{l, k}=\frac{\rho_{l, k} / m_{v, k}}{\sum_{m=1}^{N_{v}} \rho_{l, m} / m_{v, m}} .
$$

Alternatively, the molar fraction of component $k$ in an $\mathbf{n}$-droplet can be written more concisely as:

$$
x_{\mathbf{n}, k}=\frac{n_{k}}{\sum_{m=1}^{N_{v}} n_{m}} .
$$




\subsubsection{Mixture thermodynamics}

The mixture of carrier gases and vapors is considered to behave as a perfect gas, which means that the thermal and caloric equations of state presented in Eqs. (2.54) and (2.55) are also valid here for the various gaseous constituents. Based on the assumed perfect gas behavior, and neglecting the pressure contribution of the droplet dispersion, the pressure within the mixture is again equal to the sum of the partial pressures of each gaseous constituent. Eq. (2.66) is therefore also valid here, with the only difference being that the mixture gas constant is now given by:

$$
R=\sum_{k=1}^{N_{c}} g_{c, k} R_{c, k}+\sum_{k=1}^{N_{v}} g_{v, k} R_{v, k}
$$

The specific internal energy $e$ of the mixture consists of the weighted sum of the internal energies associated with each constituent,

$$
e=\sum_{k=1}^{N_{c}} g_{v, k} e_{c, k}(T)+\sum_{k=1}^{N_{v}} g_{v, k} e_{v, k}(T)+\sum_{\mathbf{n} \in \Omega_{\mathbf{N}}} \frac{\hat{\rho}_{\mathbf{n}}}{\rho} E_{\mathbf{n}}(T) .
$$

The energy $E_{\mathbf{n}}$ of a droplet can be written in a similar form as was used in the single component case, viz. Eq. (2.69), which yields the following expression:

$$
E_{\mathbf{n}}=m_{\mathbf{n}}\left[h_{v, \mathbf{n}}^{s}(T)-\frac{p}{\rho_{\mathbf{n}}(T)}-L_{\mathbf{n}}(T)\right]
$$

By neglecting the term $p / \rho_{\mathbf{n}}$ in Eq. (2.124), the expression for the specific internal energy of the mixture becomes:

$$
e=\sum_{k=1}^{N_{c}} g_{c, k} e_{c, k}(T)+\sum_{k=1}^{N_{v}} g_{v, k} e_{v, k}(T)+\sum_{\mathbf{n} \in \Omega_{\mathbf{n}}} \check{\rho}_{\mathbf{n}} m_{\mathbf{n}}\left[h_{v, \mathbf{n}}^{s}(T)-L_{\mathbf{n}}(T)\right],
$$

where $\breve{\rho}_{\mathbf{n}}=\hat{\rho}_{\mathbf{n}} / \rho$ is the specific number density. Eq. (2.125) is the caloric equation of state for the multi-component two-phase mixture, which implicitly gives the temperature $T$ as function of the mixture specific internal energy $e$, the multi-component size distribution $\hat{\rho}_{\mathbf{n}}$, and the various vapor and carrier gas composition variables. The equation of state is completed by specifying a suitable expression for $L_{\mathbf{n}}(T)$, and by setting:

$$
h_{v, \mathbf{n}}^{s}(T)=h_{v, \mathbf{n}}(T)=\sum_{k=1}^{N_{v}} w_{\mathbf{n}, k} C_{p, v, k} T
$$

Similarly to Eq. (2.125), the mixture enthalpy is expressed as:

$$
h=\sum_{k=1}^{N_{c}} g_{c, k} h_{c, k}(T)+\sum_{k=1}^{N_{v}} g_{v, k} h_{v, k}(T)+\sum_{\mathbf{n} \in \Omega_{\mathbf{n}}} \check{\rho}_{\mathbf{n}} m_{\mathbf{n}}\left[h_{v, \mathbf{n}}^{s}(T)-L_{\mathbf{n}}(T)\right] .
$$


Using Eq. (2.126) in combination with the caloric equation of state for ideal gases, the mixture enthalpy can be concisely expressed as:

$$
h=C_{p, 0} T-\sum_{\mathbf{n} \in \Omega_{\mathbf{n}}} \check{\rho}_{\mathbf{n}} m_{\mathbf{n}} L_{\mathbf{n}}(T),
$$

where:

$$
C_{p, 0}=\sum_{k=1}^{N_{c}} g_{c, k} C_{p, c, k}+\sum_{k=1}^{N_{v}} g_{v, k}^{\max } C_{p, v, k}
$$

is the specific heat of the mixture in the dry state.

\subsubsection{Mixture transport equations}

Adopting the same assumptions as have been made for the case of single component condensation, it is straightforward to derive that the mass, momentum, and energy equations for the multi-component two-phase mixture (in conservation form) are identical to Eqs. (2.84), (2.86), and (2.87), respectively. The transport equation for the multi-component size distribution can be written in a similar form as Eq. (2.82), with the condensation source term replaced with $S_{\mathbf{n}}$. This source term will be further elaborated in chapter 5 .

With respect to the characteristic form of the mixture transport equations, it can be derived that these are identical to Eqs. (2.99), (2.100), and (2.101) for the single component case, when the single-component size distribution $\check{\rho}_{n}$ is replaced with $\check{\rho}_{\mathbf{n}}$. Eq. (2.98) for the frozen speed of sound also remains valid, and only the expressions for the partial derivatives $\partial p / \partial \rho$ and $\partial p / \partial e$ need to be modified. Eqs. (2.114), (2.115), (2.119), (2.120), and (2.122) reveal that $R=R(\check{\rho})$, with $\check{\rho}=\left(\check{\rho}_{(1,1)}, \check{\rho}_{(2,0)}, . ., \check{\rho}_{(N, N)}\right)^{T}$, and therefore, Eqs. (2.103) and (2.104) also remain valid. The problem is thus again reduced to finding the partial derivatives of $T$ with respect to $\rho$ and $e$. From Eq. (2.125) it is observed that $T=T(e, \check{\rho})$, so that immediately $\partial T / \partial \rho=0$. To find $\partial T / \partial e$, Eq. (2.125) is first differentiated with respect to $e$, and with subsequent manipulations it is derived that:

$$
\frac{\partial T}{\partial e}=\left\{\sum_{k=1}^{N_{c}} g_{c, k} C_{v, c, k}+\sum_{k=1}^{N_{v}} g_{v, k} C_{v, v, k}+\sum_{\mathbf{n} \in \Omega_{\mathbf{n}}} \check{\rho}_{\mathbf{n}} m_{\mathbf{n}}\left[\sum_{k=1}^{N_{v}} w_{\mathbf{n}, k} C_{p, v, k}-\frac{\partial L_{\mathbf{n}}}{\partial T}\right]\right\}^{-1} .
$$

This completes the two-phase mixture model for multi-component condensing flow. 


\section{EVALUATION OF MASTER EQUATIONS IN CONDENSING FLOW}

The kinetic equation (KE), and its first- and second-order approximations, the general dynamic equation (GDE) and the Fokker-Planck equation (FPE), respectively, are evaluated based on (a) their equilibrium distributions, (b) a nucleation pulse experiment, and (c) an expanding nozzle flow. Large differences are observed between the equilibrium distributions of the FPE and KE, whereas the GDE does not have an equilibrium distribution at all. For the nucleation pulse experiment, good agreement is found between the KE, FPE and GDE, due to quasisteady nucleation. For the condensing nozzle flow, the difference between the GDE-and the KE-distributions is large, although the relevant flow variables show fair agreement. A sensitivity study of the KE-solution with respect to uncertainties in (a) the surface tension model, (b) the sticking probability, and $(c)$ the equilibrium distribution, revealed that both the sticking probability and the equilibrium distribution have a significant influence on the predicted condensation onset. Furthermore, it is found that the proposed Wölk and Strey-corrected Courtney equilibrium distribution yields the best agreement with reported measurements.

The work in this chapter has been published in revised form as: R.S.R. Sidin, R. Hagmeijer, and U. Sachs, "Evaluation of master equations for the droplet size distribution in condensing flow", Phys. Fluids 21, 7 (2009).

\subsection{Introduction}

Flow induced condensation occurs in various industrial applications, ranging from steam turbines to supersonic gas conditioners. In such devices, the rapid flow expansion leads to the spontaneous formation of a dispersion of small liquid droplets, also referred to as clusters. The spatial and temporal evolution of the droplet size distribution is governed by a balance equation for the droplet number density, and is referred to as either the master equation [52], the general dynamic equation [101], 
or the population balance equation [138]. The formulation of the master equation is based on the pioneering work by, amongst others, Becker and Döring [13], and the Szilard model of condensation [52], in which the growth and evaporation of droplets proceeds via the interaction with single molecules (monomers). As this process is kinetically driven, the master equation is referred to as the kinetic equation (KE). In condensation modelling, the KE is primarily used to derive expressions for the steady state nucleation rate, i.e., the rate at which nuclei are formed at the onset of condensation [52], [127]. It will be shown that the KE can be exactly represented by a partial differential equation of which the general dynamic equation (GDE) is a first-order approximation, and the Fokker-Planck (FPE) equation is a second-order approximation. Each of these equations can be ranked based on a hierarchy of accuracy, with the KE and GDE formally providing the most and least accurate descriptions of the condensation process, respectively (see Figure 3.1).

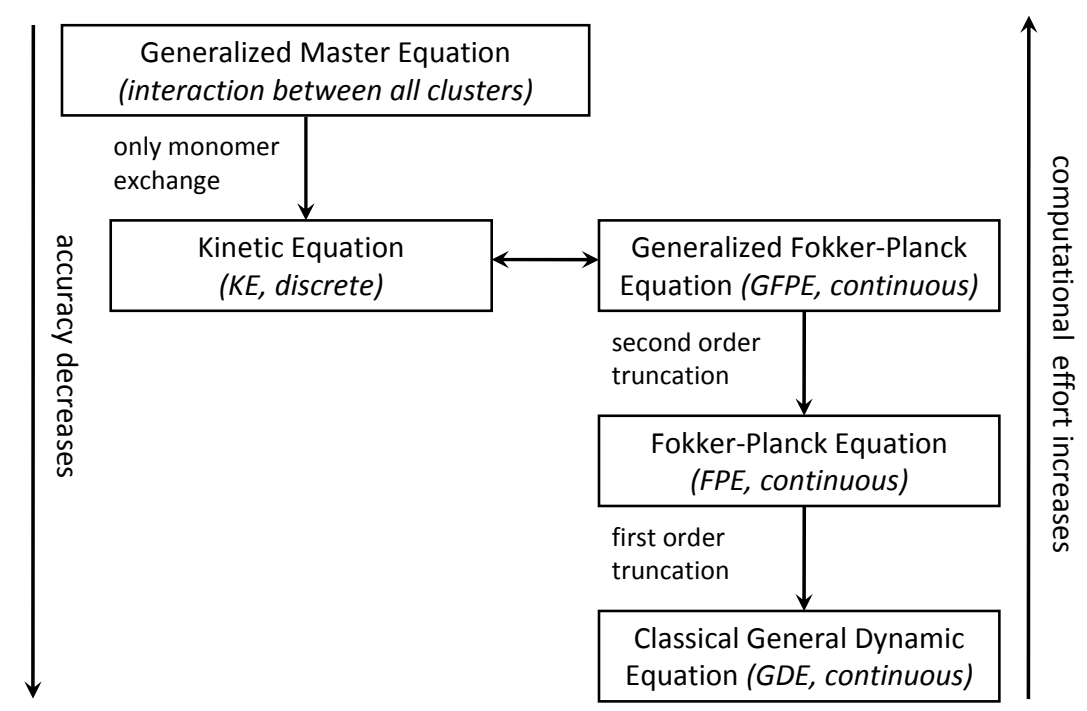

FIGURE 3.1: Hierarchy of master equations with respect to accuracy and computational demands.

Recently, Holten and van Dongen [46] have investigated the accuracy of the GDE by numerical simulation of a nucleation pulse experiment with a one-way coupling between the thermodynamic variables and the droplet size distribution. They observed good overall agreement between the KE and GDE solutions, except for some minor details in the shape of the distribution function.

In a separate investigation, White and Young [133] have also investigated the accuracy of the GDE by numerical simulation of a superheated steam expansion, but 
with a two-way coupling between the thermodynamic variables and the droplet size distribution. They observed that the agreement between the KE and GDE was poor for fast expansion rates, due to failure of the quasi-steady condensation assumption on which the GDE is based.

The objective of the present paper is to extend these investigations by employing two test cases: (a) a nucleation pulse experiment using one-way coupling, and (b) a rapidly expanding flow through a Laval nozzle using two-way coupling, for which experimental data is available. In the first test case the FPE is included as an additional candidate, and the validity of the quasi-steady nucleation assumption is addressed. The truncation errors associated with the FPE and GDE are calculated to explain the discrepancies between the predicted size distributions. In the second test case, KE and GDE results are also compared with experimental data. The validity of the physical model behind the KE is evaluated, and uncertainties in some key variables are analyzed and quantified.

\subsection{Master equations for the droplet size distribution}

The master equation is essentially a mass conservation law for droplets consisting of an integer number of vapor molecules. In the flows considered in this chapter, the concentration of vapor molecules (monomers) is much higher than that of droplets, which justifies the assumption that droplets only interact with monomers: each droplet may capture or expel a single monomer at a time. This is the so-called Szilard approach in condensation [52], for which three physical-mathematical models will be discussed in the following sections.

\subsubsection{The Kinetic Equation (KE)}

The KE for a quiescent vapor (e.g., [52], [127]) is:

$$
\frac{d \hat{\rho}_{n}}{d t}=J_{n-1}-J_{n} \quad, \quad n=2,3, . .
$$

where $\hat{\rho}_{n}$ denotes the volumetric number density of droplets consisting of $n$-molecules ( $n$-mers), and where the condensation flux $J_{n}$ contains the combined effect of a condensation (forward) rate $f_{n}$ and an evaporation (backward) rate $b_{n}$ :

$$
J_{n}=f_{n} \hat{\rho}_{n}-b_{n+1} \hat{\rho}_{n+1} .
$$

Schematically, the KE can be represented as a series of chemical reactions, as depicted in Figure 3.2. For an advected vapor, with the average advection velocity of the droplets equal to the velocity $\mathbf{u}$ of the carrier gas, the KE becomes

$$
\frac{\partial \hat{\rho}_{n}}{\partial t}+\frac{\partial}{\partial x_{j}}\left(u_{j} \hat{\rho}_{n}\right)=J_{n-1}-J_{n} \quad, \quad n=2,3, \ldots
$$



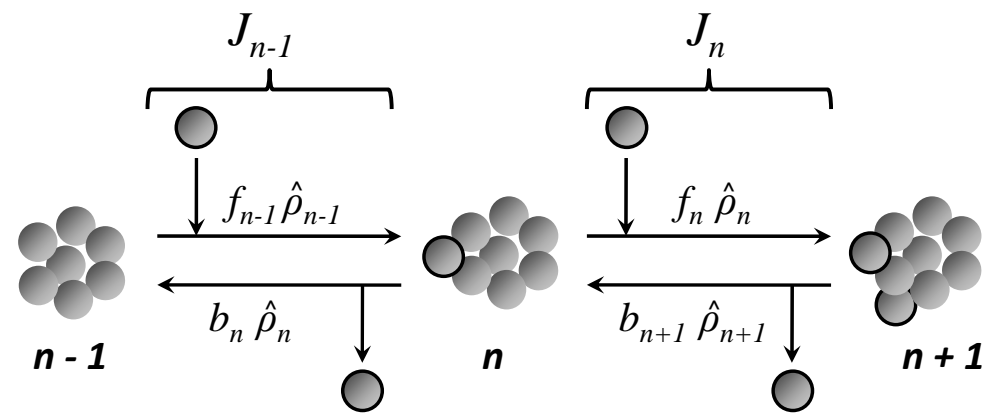

FIGURE 3.2: Schematic representation of the Szilard model for single-component condensation.

Assuming Maxwellian velocity distributions for the monomers and droplets, the forward rate $f_{n}$ may be written as [52]:

$$
f_{n}=\alpha_{n} c_{n} v_{1}^{2 / 3} \sqrt{\frac{n+1}{n}}\left(n^{1 / 3}+1\right)^{2} \sqrt{\frac{k_{B} T}{2 \pi m_{1}}} \hat{\rho}_{1}
$$

where $\alpha_{n}$ denotes the sticking probability, $c_{n}$ is the shape factor, $v_{1}$ and $m_{1}$ denote the effective volume and mass of a single molecule, respectively, $k_{B}$ denotes the Boltzmann constant, and $T$ the gas/vapor temperature. It is noted that the asymptotic size dependency of $f_{n} \sim n^{2 / 3}$ used in [46] and [133], is valid for sufficiently large droplets only.

Although the sticking probability $\alpha_{n}$ is usually set to unity, a number of recent investigations employing advanced simulation techniques have shown that $\alpha_{n}$ varies strongly with $n$ for small droplets, and that in the limit of very large droplets, $\alpha_{n}$ does not necessarily approach unity [99]. Okada and Hara [82] report values of $\alpha_{\infty}$ of about 0.6 to 1.0 , whereas $\alpha_{2}$ reaches values of as low as 0.01 (see Figure 3.3). To study the influence of $\alpha_{n}$ on the condensation process, the following model expression for $\alpha_{n}$ is used in the present investigation:

$$
\alpha_{n}=\alpha_{\infty}-\left(\alpha_{\infty}-\alpha_{1}\right) \exp \left(\frac{1-n}{n_{\infty}}\right)
$$

which roughly mimics the trends in $\alpha_{n}$ observed in [82]. The values of $\alpha_{1}, \alpha_{\infty}$, and $n_{\infty}$ will be varied to investigate their influence on the predictions.

The shape factor $c_{n}$ is set to $c_{n}=(36 \pi)^{1 / 3}$, which corresponds to spherical droplets. Although small droplets may be far from spherical (see Figure 3.4, [145]), a more accurate expression cannot be derived due to the lack of suitable data. 


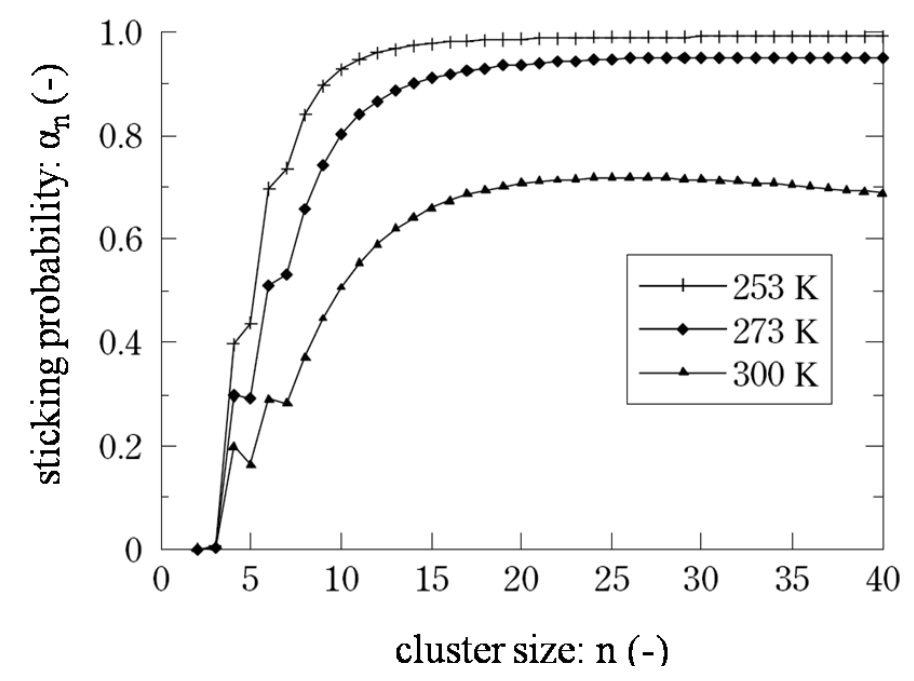

FiguRE 3.3: Sticking probability for water droplets predicted by Okada and Hara [82].

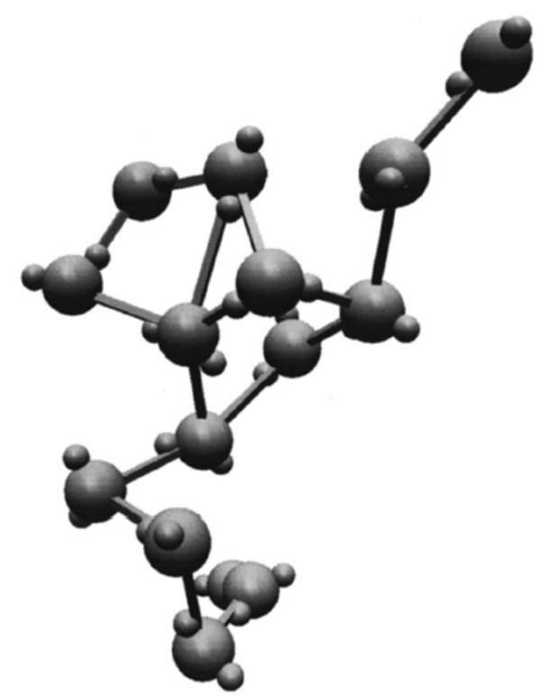

FIGURE 3.4: Shape of a water droplet with size $n=15$, as obtained from molecular dynamics simulations by Yasuoka et al., [145]. The hydrogen and oxygen atoms are represented by the small spheres and large spheres, respectively; the rods indicate hydrogen bonds. 
The backward rate $b_{n}$ is obtained from the requirement that the fluxes $J_{n}$ are zero at equilibrium and from the usual assumption that the backward rates are independent of the size distribution:

$$
b_{n}=f_{n-1} \frac{\rho_{n-1}^{e q}}{\rho_{n}^{e q}},
$$

where the superscript ' $e q$ ' refers to equilibrium conditions.

The general form of the equilibrium distribution is given by [52]:

$$
\rho_{n}^{e q}=\rho_{1}^{s} \exp \left[-\frac{\Delta G_{n}}{k_{B} T_{n}}\right],
$$

where $\Delta G_{n}$ denotes the Gibbs free energy of a droplet of size $n$, and $\rho_{1}^{s}$ is the monomer number density at saturation. The droplet temperature $T_{n}$ is assumed to be equal to the gas/vapor temperature $T$, corresponding to an isothermal condensation model. The Gibbs free energy $\Delta G_{n}$ depends on the saturation ratio $S$ (see Eq. (1.1)), and the dimensionless surface energy

$$
\theta \equiv \frac{A_{1} \sigma}{k_{B} T_{n}}
$$

where $A_{1}$ is the molecular surface area, and $\sigma$ the surface tension.

Adopting the nomenclature used by Wilemski [134], the following models for $\Delta G_{n}$ will be considered in this investigation:

1. $\Delta G_{n}$ for the Courtney distribution:

$$
-\frac{\Delta G_{n}^{C}}{k_{B} T_{n}}=n \ln S-\theta n^{2 / 3}, n>1,
$$

2. $\Delta G_{n}$ for the Self Consistent Classical distribution (SCC):

$$
-\frac{\Delta G_{n}^{S C C}}{k_{B} T_{n}}=n \ln S-\theta\left(n^{2 / 3}-1\right)
$$

3. $\Delta G_{n}$ for the Kelvin distribution (derived for a droplet in stable equilibrium):

$$
-\frac{\Delta G_{n}^{K}}{k_{B} T_{n}}=n \ln S-\frac{2}{3} \theta \sum_{j=2}^{n} j^{-1 / 3} .
$$

In addition, a fourth equilibrium distribution will be employed which combines the Courtney equilibrium distribution with the empirical correction factor for the nucleation rate reported by Wölk and Strey in [139]:

$$
\frac{J_{\text {exp }}}{J_{C N T}}=\exp \left[A+\frac{B}{T}\right] .
$$


In this expression $J_{\text {exp }}$ is the nucleation rate obtained from experiment, $J_{C N T}$ is obtained from Classical Nucleation Theory (see appendix B), and $A$ and $B$ are fluid dependent constants. The nucleation rate $J$ is generally expressed as:

$$
J=K \exp \left(-\frac{\Delta G_{n^{*}}}{k_{B} T}\right),
$$

where $n^{*}$ denotes the critical droplet size and $K$ the kinetic prefactor (see appendix B). Multiplication of $J_{C N T}$ by the empirical correction factor may be interpreted as a correction to $\Delta G_{n^{*}}^{C}$ :

$$
\Delta G_{n^{*}}=\Delta G_{n^{*}}^{C}-\left(A+\frac{B}{T}\right) k_{B} T_{n} .
$$

Application of the correction to all droplet sizes finally leads to:

4. $\Delta G_{n}$ for the Wölk and Strey corrected Courtney distribution (CWS):

$$
-\frac{\Delta G_{n}^{C W S}}{k_{B} T_{n}}=n \ln S-\theta n^{2 / 3}+A+\frac{B}{T}, n>1 .
$$

\subsubsection{The Fokker-Planck Equation (FPE)}

By replacing the discrete distribution $\rho_{n}(\mathbf{x}, t)$ with a continuous distribution $\hat{\rho}(n, \mathbf{x}, t)$, where $\hat{\rho}(n, \mathbf{x}, t)=\rho_{n}$ for $n \in \mathbb{N}^{+}$, and applying a Taylor series expansion to the right-hand side of Eq. (3.3), the following generalized Fokker-Planck equation [95] is derived:

$$
\frac{\partial \hat{\rho}}{\partial t}+\frac{\partial}{\partial x_{j}}\left(u_{j} \hat{\rho}\right)=\sum_{k=1}^{\infty} T^{k}(n),
$$

where:

$$
T^{k}(n) \equiv \frac{1}{k !} \frac{\partial^{k}}{\partial n^{k}}\left[\left(f_{n}(-1)^{k}+b_{n}\right) \hat{\rho}(n)\right] .
$$

It is noted that Eq. (3.16) is an exact representation of Eq. (3.3). By truncating Eq. (3.16) up to the second-order term $(k=2)$, the Fokker-Planck equation (FPE) is obtained:

$$
\frac{\partial \hat{\rho}}{\partial t}+\frac{\partial}{\partial x_{j}}\left(u_{j} \hat{\rho}\right)=-\frac{\partial}{\partial n}(\dot{n} \hat{\rho})+\frac{\partial^{2}}{\partial n^{2}}(D \hat{\rho}) \text {. }
$$

A similar equation was derived by Tunitskii [52]. The drift velocity $\dot{n}=\dot{n}(n, \mathbf{x}, t)$ is given by:

$$
\dot{n}=f_{n}-b_{n},
$$

which resembles the average growth rate of the droplets. The diffusion coefficient $D=D(n, \mathbf{x}, t)$ is

$$
D=\frac{1}{2}\left(f_{n}+b_{n}\right)
$$


which reflects the statistical nature of the droplet formation process. It is noted that by using the FPE, a third order truncation error is introduced with respect to the KE. As a consequence of the fixed step size used in the Taylor expansion, i.e., $\Delta n=1$, the truncation error is also fixed, which means that good agreement between Eqs. (3.3) and (3.18) is not guaranteed.

The equilibrium distribution $\hat{\rho}^{e q}(n)$ of the FPE satisfies:

$$
\dot{n} \hat{\rho}^{e q}-\frac{d}{d n}\left(D \hat{\rho}^{e q}\right)=0
$$

subject to the boundary condition:

$$
\hat{\rho}^{e q}\left(n_{0}\right)=\hat{\rho}_{0}^{e q},
$$

with $n_{0} \geq 2$ corresponding to the smallest droplet accounted for in the FPE. Rearrangement of Eq. (3.21), i.e.,

$$
\frac{d \hat{\rho}^{e q}}{d n}+\left[\frac{1}{D(n)} \frac{d D(n)}{d n}-\frac{\dot{n}(n)}{D(n)}\right] \hat{\rho}^{e q}=0
$$

leads to the Fokker-Planck equilibrium distribution

$$
\hat{\rho}^{e q}(n)=\hat{\rho}_{0}^{e q} \frac{D\left(n_{0}\right)}{D(n)} \exp \left[\int_{n_{0}}^{n} \frac{\dot{n}(\xi)}{D(\xi)} d \xi\right] .
$$

It is noted that for large droplets

$$
\frac{\dot{n}(n)}{D(n)} \rightarrow 2 \frac{S-1}{S+1},
$$

which, in a supersaturated state $(S>1)$, leads to a non-physical equilibrium for large droplets.

The relative importance of the diffusion term compared to the drift term can be quantified by means of a droplet Péclet number $P e ́(n)$, defined as:

$$
P e ́(n) \equiv \frac{\dot{n}(n) n}{D(n)}
$$

which gives an order-of-magnitude comparison between the first and second order term. As shown in Eq. (3.25), the ratio between $\dot{n}(n)$ and $D(n)$ reduces to a constant in the limit of very large droplets, and therefore: $P e ́(n) \sim n$ for $n \gg 1$. Thus, it is concluded that the diffusion term can be neglected in the large-droplet limit. 


\subsubsection{The General Dynamic Equation (GDE)}

Computational models for condensing flow, e.g., [45], [42], [131], [68], are frequently based on the so-called general dynamic equation (GDE), which is a first-order approximation of the KE:

$$
\frac{\partial \hat{\rho}}{\partial t}+\frac{\partial}{\partial x_{j}}\left(u_{j} \hat{\rho}\right)+\frac{\partial}{\partial n}(\dot{n} \hat{\rho})=0 .
$$

The use of the GDE instead of the more elaborate KE, or generalized FPE is justified when the higher order terms in Eq. (3.16) are much smaller than the first order term. Indeed, by using a quasi-steady state solution for the droplet size distribution, Muitjens [79] has shown that the drift term in the FPE becomes dominant over the diffusion term for supercritical droplet sizes, i.e., for $n>n^{*}$. Muitjens suggests that for $n \geq n_{0}, n_{0}=2 n^{*}$, the diffusion flux may be neglected. A similar suggestion was made by Hill [45], who introduced a droplet source term at $r_{0}=1.3 r^{*}$ based on the observation that at that droplet size the probability of growth is almost unity. As a result, the GDE is only applicable for $n>n_{0}$, and a higher-order approximation of the $\mathrm{KE}$ would be required to cover the time dependent range $2 \leq n<n_{0}$. It is one of the objectives of the present investigation to determine whether or not the above stated assumptions hold in condensation problems with rapid expansions.

In computational models employing the GDE, the usual practice is to neglect all droplets smaller than $n_{0}$ :

$$
\hat{\rho}(n)=0, \quad 2 \leq n<n_{0} .
$$

When $n_{0}$ is constant, which occurs in quasi-steady nucleation, the flux at $n=n_{0}$ can conveniently be specified in the form:

$$
\left.\dot{n} \hat{\rho}\right|_{n=n_{0}}=J,
$$

where $J$ is the nucleation rate which is assumed to be independent of $n$ for $2 \leq n \leq n_{0}$. When $n_{0}$ varies with time, however, the boundary condition in Eq. (3.29) needs to be replaced by [68]:

$$
\left.\Delta \dot{n} \hat{\rho}\right|_{n=n_{0}}=J, \quad \Delta \dot{n} \equiv \dot{n}-\frac{D n_{0}}{D t},
$$

with $D / D t$ representing the substantial derivative. Boundary condition Eq. (3.30) can only be applied as long as $\Delta \dot{n}>0$, since when $\Delta \dot{n}<0$ droplets are leaving the computational domain and a boundary condition is not required. This situation resembles instantaneous evaporation, and is actually similar to the denucleation mechanism which was mentioned by Hagmeijer et al. in [42] and [107], where $n_{0}=n^{*}$. It is noted that in case of rapid expansions the quasi steady nucleation assumption, i.e., the assumption that $J$ is independent of $n$ for $2 \leq n \leq n_{0}$, may loose validity and may lead to deviations between the solutions of the GDE and the KE. 
By defining the radius distribution function as

$$
f\left(r\left(n, r_{1}\right), \mathbf{x}, t\right)=\frac{3 n}{r\left(n, r_{1}\right)} \hat{\rho}(n, \mathbf{x}, t),
$$

with $r=r_{1} n^{1 / 3}$, and $r_{1}=r_{1}(\mathbf{x}, t)$ denoting the effective monomer radius, the following general dynamic equation for the droplet radius distribution function is derived from Eq. (3.27):

$$
\frac{\partial f}{\partial t}+\frac{\partial}{\partial x_{j}}\left(u_{j} f\right)+\frac{\partial}{\partial r}\left(\dot{r}_{K E} f\right)=0
$$

The growth rate $\dot{r}_{K E}$ is given by:

$$
\dot{r}_{K E}=\dot{n} \frac{\partial r}{\partial n}+\frac{D r_{1}}{D t} \frac{r}{r_{1}} .
$$

The first term on the right-hand side of Eq. (3.33) represents the change in droplet radius due to mass accumulation, whereas the second term resembles the effect of compression or expansion of the liquid phase. The subscript $K E$ in $\dot{r}_{K E}$ indicates that the growth of droplets is governed by the kinetics of monomer impingement. It can be shown that for sufficiently large droplets and incompressible liquids (i.e., $D r_{1} / D t=0$ ), the growth rate $\dot{r}_{K E}$ reduces to the well known Hertz-Knudsen droplet growth law.

It is noted that the boundary condition Eq. (3.30) is traditionally implemented into Eq. (3.27) by adding a source term which includes a Dirac delta function. The equation thus obtained is similar in form to Eq. (3.27), and is given by:

$$
\frac{\partial \hat{\rho}}{\partial t}+\frac{\partial}{\partial x_{j}}\left(u_{j} \hat{\rho}\right)+\frac{\partial}{\partial n}(\dot{n} \hat{\rho})=J \delta\left(n-n_{0}\right) .
$$

To show that the formulation of the condensation problem given by Eq. (3.34) is equivalent to Eq. (3.27), with Eq. (3.30) specified as boundary condition, a general solution for both problems is independently derived by means of the method of characteristics. Here, a characteristic, denoted by $\overline{\mathbf{x}}(t), \bar{n}(t)$, represents a trajectory in $(\mathbf{x}, n)$-space, which follows from the solution of the coupled initial value problem:

$$
\begin{array}{lll}
\frac{d \overline{\mathbf{x}}}{d t}=\mathbf{u}(\overline{\mathbf{x}}(t), t) & , & \overline{\mathbf{x}}\left(t_{1}\right)=\mathbf{x}_{1} \\
\frac{d \bar{n}}{d t}=\dot{n}(\overline{\mathbf{x}}(t), \bar{n}(t), t) & , & \bar{n}\left(t_{1}\right)=n_{1},
\end{array}
$$

where $\left(\mathbf{x}_{1}, n_{1}\right)$ denotes a point through which the characteristic passes at an arbitrary reference time $t_{1}$. Starting with Eq. (3.34), the nucleation source term on the righthand side is first replaced by the expression:

$$
J \delta\left(n-n_{0}\right)=J(t) \frac{\delta\left(t-t_{0}\right)}{\left|\dot{n}-\frac{d n_{0}}{d t}\right|(t)},
$$


where $\bar{n}\left(t_{0}\right)=n_{0}$. By introducing the derivative $d / d t$, where:

$$
\frac{d(.)}{d t} \equiv \frac{\partial(.)}{\partial t}+u_{j} \frac{\partial(.)}{\partial x_{j}}+\dot{n} \frac{\partial(.)}{\partial n},
$$

and replacing $\hat{\rho}_{n}$ with the specific number density $\check{\rho}_{n}=\hat{\rho}_{n} / \rho$, Eq. (3.34) may be rewritten along a characteristic as:

$$
\frac{d \check{\rho}}{d t}+\check{\rho} \frac{\partial \dot{n}}{\partial n}=\frac{J(t)}{\rho(t)} \frac{\delta\left(t-t_{0}\right)}{\left|\dot{n}-\frac{d n_{0}}{d t}\right|(t)} .
$$

Multiplication of Eq. (3.39) with an integrating factor $a(t)$, where:

$$
a(t)=\exp \left[\int_{0}^{t} \frac{\partial \dot{n}}{\partial n}\left(\bar{n}\left(t^{\prime}\right), t^{\prime}\right) d t^{\prime}\right]
$$

followed by integration in time, yields the following solution:

$$
\left[\check{\rho}\left(\bar{n}\left(t^{\prime}\right), t^{\prime}\right) a\left(t^{\prime}\right)\right]_{t_{0}(1-\beta)}^{t}=\int_{t_{0}(1-\beta)}^{t} a\left(t^{\prime}\right) \frac{J\left(t^{\prime}\right)}{\rho\left(t^{\prime}\right)} \frac{\delta\left(t^{\prime}-t_{0}\right)}{\left|\dot{n}-\frac{d n_{0}}{d t}\right|\left(t^{\prime}\right)} d t^{\prime},
$$

where $\beta$ is an arbitrary positive number smaller than unity, such that $\bar{n}\left(t_{0}(1-\beta)\right)<n_{0}$. As $\breve{\rho}(n)=0$ for $n<n_{0}$, and because the Dirac delta-function filters the integrand in Eq. (3.41) at $t=t_{0}$, the final solution for Eq. (3.34) is given by:

$$
\check{\rho}(\bar{n}(t), t)=\frac{a\left(t_{0}\right)}{a(t)} \frac{J\left(t_{0}\right)}{\rho\left(t_{0}\right)\left|\dot{n}-\frac{d n_{0}}{d t}\right|\left(t_{0}\right)} .
$$

The nucleation flux $J$ is only specified when $\dot{n}-\frac{d n_{0}}{d t}>0$, and therefore, the absolute signs on the right-hand side of Eq. (3.42) can be dropped:

$$
\check{\rho}(\bar{n}(t), t)=\frac{a\left(t_{0}\right)}{a(t)} \frac{J\left(t_{0}\right)}{\rho\left(t_{0}\right)\left(\dot{n}-\frac{d n_{0}}{d t}\right)\left(t_{0}\right)} .
$$

The next step is to obtain the solution of Eq. (3.27), with the boundary condition given by Eq. (3.30). By applying similar mathematical manipulations as performed above, the following solution of Eq. (3.27) (along a characteristic) is derived:

$$
\left[\check{\rho}\left(\bar{n}\left(t^{\prime}\right), t^{\prime}\right) a\left(t^{\prime}\right)\right]_{t_{0}}^{t}=0 .
$$

Eq. (3.44) is similar to Eq. (3.41), except for the nucleation source term and the lower integration boundary. Since: $\bar{n}\left(t_{0}\right)=n_{0}$, and: $d n_{0} / d t=D n_{0} / D t$ due to $n_{0}=n_{0}(\mathbf{x}, t)$, the boundary condition given in Eq. (3.30) allows $\breve{\rho}\left(\bar{n}\left(t_{0}\right), t_{0}\right)$ to be expressed as:

$$
\check{\rho}\left(\bar{n}\left(t_{0}\right), t_{0}\right)=\frac{J\left(t_{0}\right)}{\rho\left(t_{0}\right)\left(\dot{n}-\frac{d n_{0}}{d t}\right)\left(t_{0}\right)} .
$$


Substitution of Eq. (3.29) into Eq. (3.44) then yields:

$$
\check{\rho}(\bar{n}(t), t)=\frac{a\left(t_{0}\right)}{a(t)} \frac{J\left(t_{0}\right)}{\rho\left(t_{0}\right)\left(\dot{n}-\frac{d n_{0}}{d t}\right)\left(t_{0}\right)} .
$$

This is exactly equal to the solution of Eq. (3.34) in Eq. (3.43), thus completing the proof for equivalence of both formulations.

It is finally noted that the GDE, in contrast to the KE and the FPE, does not allow for an equilibrium distribution: subcritical droplets will always evaporate, whereas supercritical droplets will always grow.

\subsection{Evaluation method}

\subsubsection{Description of test cases}

In the first test case, a nucleation pulse experiment with water vapor is simulated, similar to the one used by Holten and van Dongen [46]. The KE (Eq. (3.3)), the FPE (Eq. (3.18)), and the GDE (Eq. (3.32)) are solved for the temperature and saturation histories given in Figure 3.5, with $u_{i}=0, i=1,2,3$. The simulation is one-way coupled, i.e., the effects of latent heat release and vapor depletion are not accounted for. The predicted size distributions obtained with the various master equations are compared, and the condensation fluxes and truncation errors are analyzed to explain observed differences.

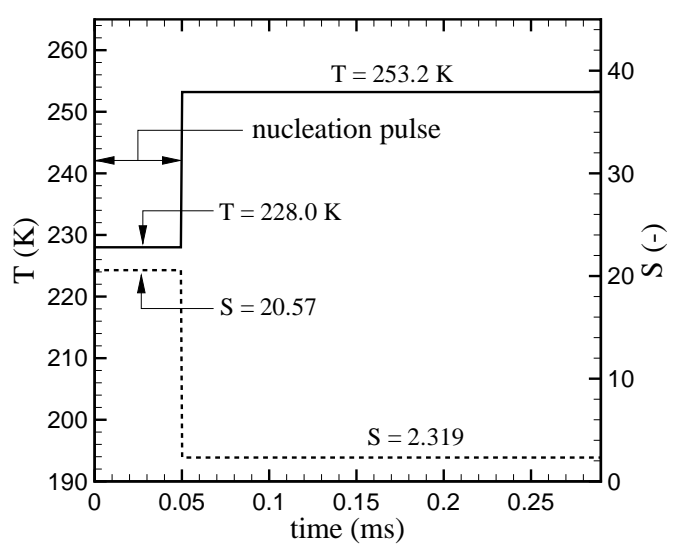

FIGURE 3.5: Temperature and saturation ratio evolution in the nucleation pulse experiment.

In the second test case, the condensing flow through a Laval nozzle is simulated, corresponding with experiments conducted by Tanimura et al. [119]. By using isen- 
tropic relations, the nozzle area variation in Figure 3.13.a has been reconstructed from the dry-flow pressure profiles given in [119] to account for boundary-layer effects. The nozzle diverges very slowly downstream of the throat, which justifies the use of a quasi-one-dimensional simulation. The experiment utilizes a mixture of nitrogen $\left(\mathrm{N}_{2}\right)$ and methane $\left(\mathrm{CH}_{4}\right)$ as the carrier gas, and heavy water $\left(\mathrm{D}_{2} \mathrm{O}\right)$ as the condensing component. The mole fraction of methane in the mixture is approximately $4 \%$, and that of $\mathrm{D}_{2} \mathrm{O}$ approximately $2.5 \%$, which corresponds with a total $\mathrm{D}_{2} \mathrm{O}$ mass fraction of $g_{\max }=0.0018$. The total pressure and temperature of the mixture at the nozzle inlet are $p_{0}=30.1 \mathrm{kPa}$ and $T_{0}=298 \mathrm{~K}$, respectively. The material properties of $\mathrm{N}_{2}, \mathrm{CH}_{4}$ and $\mathrm{D}_{2} \mathrm{O}$ are taken from [44], and given in appendix A. The latent heat of condensation is set equal to the bulk-value $L_{b}=L_{b}(T)$ for $\mathrm{D}_{2} \mathrm{O}$. A two-way coupling is utilized, accounting for the effects of vapor depletion and latent heat release. The simulation is based on solving the FDE for the mixture (Eq. (2.84), (2.86), and (2.87)) simultaneously with either the KE (Eq. (3.3)), or the GDE (Eq. (3.32)). FPE results have not been obtained for this test case since the FPE equilibrium distribution corresponds to excessively high liquid mass fractions which triggers the formation of unsteady shocks.

The influence of the sticking probability $\alpha_{n}$ is evaluated by considering Eq. (3.5) with the following variants: (i) $\alpha_{n}=1 \forall n$; (ii) $\alpha_{n}=0.8 \forall n$; (iii) $\alpha_{1}=10^{-2}, \alpha_{\infty}=1$, $n_{\infty}=10$; (iv) $\alpha_{1}=10^{-1}, \alpha_{\infty}=1, n_{\infty}=10$; (v) $\alpha_{1}=10^{-2}, \alpha_{\infty}=1, n_{\infty}=5$. The influence of the employed equilibrium size distribution on the backward rate is evaluated by comparing results obtained with Eq. (3.9), (3.10), (3.11), and (3.15). The influence of the choice of $n_{0}$ is investigated by taking

$$
n_{0}=\epsilon n^{*}, \quad 1<\epsilon \leq 2 \text {, }
$$

similar to the approach used by Holten et al. in [46]. Finally, the influence of the surface tension model is assessed by employing an additional expression for $\sigma(T)$ from [28].

Unless specified otherwise, the backward rate $b_{n}$ in the KE and FPE is based on the Courtney equilibrium distribution. By default, the forward rate is calculated by using variant (i) for the sticking probability $\left(\alpha_{n}=1\right)$. For the KE, the lower boundary is set to a default value of $n_{0}=2$. With respect to the GDE, the default nucleation rate is computed from the CNT-model, and the growth rate from the Hertz-Knudsen droplet growth law, with the lower boundary set at $r_{0}=r\left(2 n^{*}\right)$ (see appendix B).

\subsubsection{Numerical methods}

The maximum droplet radii observed in the simulations are approximately $0.1 \mu \mathrm{m}$, which corresponds with a droplet size of $n \approx 10^{8}$. To reduce the computational effort in solving the KE, droplets are grouped into bins in similar fashion as was done in 
[46] and [133] (see Figure 3.6). For droplets smaller than a certain size, a single droplet is associated with each bin, whereas for larger droplets the number of droplet sizes assigned per bin progressively increases. In this way it is possible to cover the entire range of droplet sizes by a set of 400 to 2000 bins, with the smallest droplet size equal to $n=2$, and the largest to $n=5 \cdot 10^{8}$.

The KE for the droplet bins is given by:

$$
\frac{\partial \bar{\rho}_{k}}{\partial t}+\frac{\partial}{\partial x_{j}}\left(\bar{\rho}_{k} u_{j}\right)=\frac{1}{w_{k}}\left(\bar{J}_{k-1}-\bar{J}_{k}\right)
$$

where $\bar{\rho}_{k}$ is the average number density for the $k^{\text {th }}$ bin, $w_{k}$ is the number of droplet sizes associated with bin $k$, and where $\bar{J}_{k}$ is the condensation flux between bins $k$ and $k+1$. The flux $\bar{J}_{k}$ is calculated by means of Eq. (3.2): $\bar{J}_{k}=J_{n_{r}(k)}$, with $n_{r}(k)$ denoting the largest droplet in the $k^{\text {th }}$ bin. This calculation requires the number densities at the neighboring droplet sizes of $n_{r}(k)$ and $n_{l}(k+1)$, with the latter representing the smallest droplet belonging to bin $k+1$. By applying zero-th or first-order interpolation to the bin-averaged number densities $\bar{\rho}_{k}$ and $\bar{\rho}_{k+1}$, an estimate of the number densities at the neighboring droplets can be obtained.

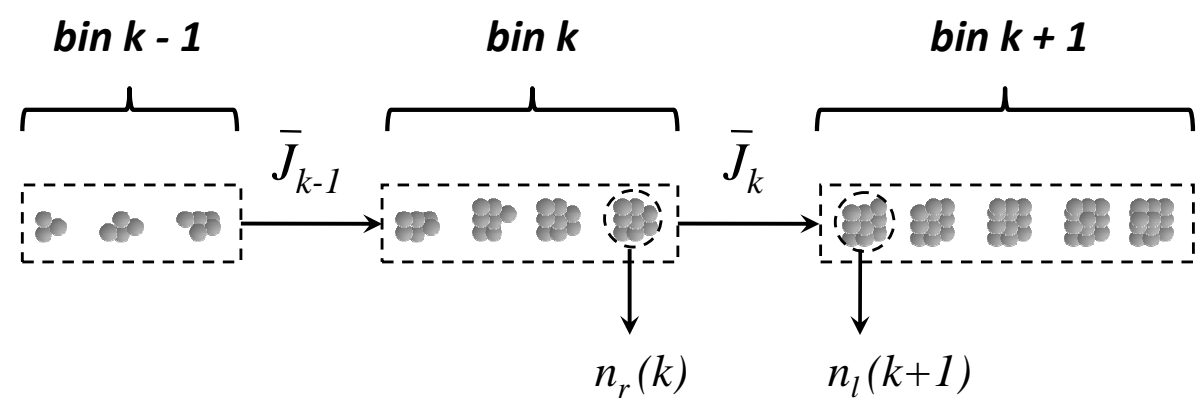

FIGURE 3.6: Grouping of droplets into bins for the numerical solution of the KE.

When solving the FPE or the GDE, the drift and diffusion terms in $n$-space need to be discretized. Hereto, the droplet size space is again divided into bins, whereafter a second-order finite volume discretization is carried out. The resulting semi-discrete form of the FPE is given by:

$$
\frac{\partial \tilde{\rho}_{k}}{\partial t}+\frac{\partial}{\partial x_{j}}\left(\tilde{\rho}_{k} u_{j}\right)=-\frac{1}{\Delta n_{k}}[\dot{n} \tilde{\rho}]_{n_{k-1 / 2}}^{n_{k+1 / 2}}+\frac{1}{\Delta n_{k}}\left[\frac{\partial}{\partial n}(D \tilde{\rho})\right]_{n_{k-1 / 2}}^{n_{k+1 / 2}}
$$

where $\tilde{\rho}_{k}$ represents the average value of $\hat{\rho}$ over bin $k$, whose edges are located at $n_{k \pm 1 / 2}$. The drift flux on the right-hand side of Eq. (3.49) is calculated by means of 
either the second-order MSOU-scheme [118] or the fifth-order WENO-scheme [106], and the diffusive flux is calculated by using a central discretization. The semi-discrete form of the GDE is similar to Eq. (3.49), except for the diffusion term.

The spatial discretization of the master equations and FDE is done by means of a finite volume method, which formally provides second-order accuracy. Introducing the vector of state variables $\mathbf{q}$, where $\mathbf{q}=\left(\rho, \rho u, \rho e_{t}, \bar{\rho}_{2}, . ., \bar{\rho}_{N-1}\right)^{T}$ for the FDE and $\mathrm{KE}$, the quasi-one-dimensional balance equation

$$
\frac{\partial \mathbf{q}}{\partial t}+\frac{1}{A} \frac{\partial}{\partial x}(\mathbf{F}(\mathbf{q}) A)=\mathbf{S}(\mathbf{q})
$$

with $A=A(x)$ the nozzle flow area, is discretized as:

$$
\frac{d \tilde{\mathbf{q}}_{i}}{d t}+\frac{1}{A_{i} \Delta x_{i}}[\mathbf{F}(\tilde{\mathbf{q}}) A]_{x_{i-1 / 2}}^{x_{i+1 / 2}}=\mathbf{S}\left(\tilde{\mathbf{q}}_{i}\right)
$$

where $\tilde{\mathbf{q}}_{i}$ represents the cell averaged value of $\mathbf{q}, \mathbf{F}$ the flux in physical space, $\mathbf{S}$ the relevant source terms, $i$ the index of the computational cell, and $\Delta x_{i}=x_{i+1 / 2}-x_{i-1 / 2}$ the corresponding cell width. For the system of FDE and FPE, or GDE, the discretized form is similar to Eq. (3.51), with $\mathbf{q}$ replaced by $\mathbf{q}=\left(\rho, \rho u, \rho e_{t}, \tilde{\rho}_{1}, . ., \tilde{\rho}_{N}\right)^{T}$. The fluxes in physical space are calculated by means of Anderson's $\kappa$-scheme, which employs van Leer flux splitting [62] and the van Albada flux limiter [2].

For the first test case, the discretized master equations are integrated in time by means of a second-order predictor-corrector method, using typical time steps of $10^{-9} \mathrm{~s}$. For the second test case, the system in Eq. (3.51) is marched in time by means of the same time-integration method, until a steady state is reached. Rewriting the semidiscrete form in Eq. (3.51) as:

$$
\frac{d \tilde{\mathbf{q}}_{i}}{d t}=\mathbf{R}_{i}+\mathbf{S}_{i}
$$

where: $\mathbf{R}_{i}=\mathbf{R}\left(\tilde{\mathbf{q}}_{i}, \tilde{\mathbf{q}}_{i \pm 1}, \tilde{\mathbf{q}}_{i \pm 2}\right)$ denotes the advection residual vector, the time integration for the FDE/FPE and FDE/GDE-systems proceeds as follows:

1. predictor step:

$$
\tilde{\mathbf{q}}_{i}^{p}=\tilde{\mathbf{q}}_{i}^{m}+\left(\mathbf{R}_{i}^{m}+\mathbf{S}_{i}^{m}\right) \Delta t^{m}
$$

2. corrector step:

$$
\tilde{\mathbf{q}}_{i}^{m+1}=\tilde{\mathbf{q}}_{i}^{m}+\frac{1}{2}\left(\mathbf{R}_{i}^{m}+\mathbf{S}_{i}^{m}+\mathbf{R}_{i}^{p}+\mathbf{S}_{i}^{p}\right) \Delta t^{m}
$$

with $m$ denoting the time level and $\Delta t^{m}$ the corresponding integration time step. For the KE, time integration proceeds in a slightly different manner, and is based on a semi-implicit adaptation of the predictor-corrector method. Denoting the state vector 
for the mean number densities by $\overline{\boldsymbol{\rho}}=\left(\bar{\rho}_{2}, . ., \bar{\rho}_{N-1}\right)^{T}$, the semi-discrete form of the $\mathrm{KE}$ is given by:

$$
\frac{d \overline{\boldsymbol{\rho}}_{i}}{d t}=\mathbf{R}_{r, i}+\mathbf{S}_{r, i}+\mathbf{A}_{d, i} \overline{\boldsymbol{\rho}}_{i},
$$

where $\mathbf{R}_{r}$ is the advection residual vector, and where the matrix $\mathbf{A}_{d}$ is given by:

$$
\mathbf{A}_{d}=\left(\begin{array}{ccccccc}
-\frac{f_{n_{r}(2)}+b_{n_{l}(2)}}{w_{2}} & \frac{b_{n_{l}(3)}}{w_{3}} & 0 & . . & 0 & 0 & 0 \\
\frac{f_{n_{r}(2)}}{w_{2}} & -\frac{f_{n_{r}(3)+b_{n_{l}(3)}}}{w_{3}} & \frac{b_{n_{l}(4)}}{w_{4}} & . . & 0 & 0 & 0 \\
: & : & : & . . & : & \vdots & \vdots \\
0 & 0 & 0 & . . & \frac{f_{n_{r}(N-3)}}{w_{N-3}} & -\frac{f_{n_{r}(N-2)+b_{n_{l}(N-2)}}}{w_{N-2}} & \frac{b_{n_{l}(N-1)}}{w_{N-1}} \\
0 & 0 & 0 & . . & 0 & \frac{f_{n_{r}(N-2)}}{w_{N-2}} & -\frac{f_{n_{r}(N-1)+b_{n_{l}(N-1)}}}{w_{N-1}}
\end{array}\right),
$$

corresponding with a zero-th order interpolation to obtain the number densities at the edges of neighboring droplet bins. The vector $\mathbf{S}_{r}$ on the right-hand side of Eq. (3.55) contains the contributions from the monomers $(n=1)$ and the largest droplets $(n=N)$, and is given by:

$$
\mathbf{S}_{r}=\left(\frac{f_{n_{r}(1)}}{w_{1}} \bar{\rho}_{1}, 0, \ldots, 0, \frac{b_{n_{l}(N)}}{w_{N}} \bar{\rho}_{N}\right)^{T} .
$$

The monomer number density is determined from the partial vapor density $\rho_{v}$ by: $\bar{\rho}_{1}=\rho_{v} / m_{1}$, whereas the number density for the largest droplet is set to zero: $\bar{\rho}_{N}=0$. By applying a first-order discretization to the time derivative in Eq. (3.55), and by evaluating the first and second term on the right-hand side of Eq. (3.55) at time $t^{m}$, whereas the last term is evaluated at the new time $t^{m+1}$, the following numerical approximation to Eq. (3.55) is obtained:

$$
\overline{\boldsymbol{\rho}}_{i}^{m+1}=\overline{\boldsymbol{\rho}}_{i}^{m}+\left(\mathbf{R}_{r, i}^{m}+\mathbf{S}_{r, i}^{m}+\mathbf{A}_{d, i}^{m} \overline{\boldsymbol{\rho}}_{i}^{m+1}\right) \Delta t^{m} .
$$

This is a linear equation for the unknown number density vector $\overline{\boldsymbol{\rho}}_{i}^{m+1}$, for which solution yields:

$$
\overline{\boldsymbol{\rho}}_{i}^{m+1}=\mathbf{C}_{i}^{m}\left[\overline{\boldsymbol{\rho}}_{i}^{m}+\left(\mathbf{R}_{r, i}^{m}+\mathbf{S}_{r, i}^{m}\right) \Delta t^{m}\right],
$$

where the matrix $\mathbf{C}_{i}^{m}$ is given by:

$$
\mathbf{C}_{i}^{m}=\left[\mathbf{I}-\mathbf{A}_{d, i}^{m} \Delta t^{m}\right]^{-1},
$$

with I denoting the identity matrix. By applying Eq. (3.59) to successive predictor and corrector steps, a second-order semi-implicit time integration method for the KE is obtained.

The convergence of the numerical solution corresponding to each test case is verified by solving the discretized equations on successively refined grids. For spatial discretization, a number of 400 grid points was found to be sufficient, whereas for the discretization in droplet size space a maximum of 2000 droplet bins has been used. 


\subsection{Results and discussion}

\subsubsection{Equilibrium distributions}

Figures 3.7. $\mathrm{a}$ and $\mathrm{b}$ show the Courtney and FPE equilibrium distributions for water vapor at (a) $T=253.2 \mathrm{~K}, S=0.5$, and (b) $T=253.2 \mathrm{~K}, S=5.0$, respectively. The sticking probability has been set to unity, and the backward rate is based on the Courtney equilibrium distribution. For the FPE, the lower boundary $n_{0}$ has been taken equal to 2,5 , and 10 respectively.

For the subsaturated vapor (Figure 3.7.a), the equilibrium distributions monotonically decrease with increasing droplet size, whereas for the supersaturated vapor (Figure 3.7.b), there is a minimum present at $r=r^{*}$. The monotonic increase in number densities of supercritical droplets $\left(r>r^{*}\right)$ cannot exist for arbitrarily large droplets, and therefore these distributions are referred to as a constrained equilibrium distributions [52], [127]. Figures 3.7 shows that there are large differences between the Courtney and FPE equilibrium distributions which are due to the truncation error $R^{2}(n)$, with:

$$
R^{k}(n) \equiv \sum_{j=k+1}^{\infty} T^{j}(n) .
$$

Comparison of Figures 3.7.a and b suggests that the influence of the truncation error on the equilibrium distributions appears to be more pronounced for lower values of the saturation. In Figure 3.8 the normalized truncation error $R^{k}(n) / R^{1}(n)$ obtained from the Courtney equilibrium distribution has been plotted for $k=1,2, . ., 10$, revealing that for small droplets (e.g., $n=5$ ), the truncation error first shows a maximum, after which it gradually reduces to zero as $k$ is further increased. For larger droplets (e.g., $n=50$ ), the truncation error decreases almost monotonically with increasing truncation order.

\subsubsection{Test case 1: nucleation pulse experiment}

The size distributions predicted by the KE, FPE and GDE are shown in Figure 3.9, for: $t=0.025 \mathrm{~ms}$ (halfway the nucleation pulse), $t=0.05 \mathrm{~ms}$ (end of the pulse), $t=0.17 \mathrm{~ms}$, and $t=0.29 \mathrm{~ms}$. For the KE the lower boundary is set at $n_{0}=5$, and for the FPE $n_{0}=15$, with $\rho_{n_{0}}$ and $\hat{\rho}\left(n_{0}\right)$ obtained from the Courtney equilibrium distribution. For the GDE, the lower boundary is set at $n_{0}=2 n^{*}$, and the initial distribution is zero everywhere. Overall, the size distributions corresponding with the KE and GDE agree with those reported in [46]. There are some minor differences associated with the GDE-solution, which stem from the use of a slightly modified growth law and the application of the GDE to subcritical droplets in [46].

Comparing the FPE-solution with the KE-solution (see Figures 3.9 and 3.12), it is observed that the FPE is able to predict the shape of the distribution function very 


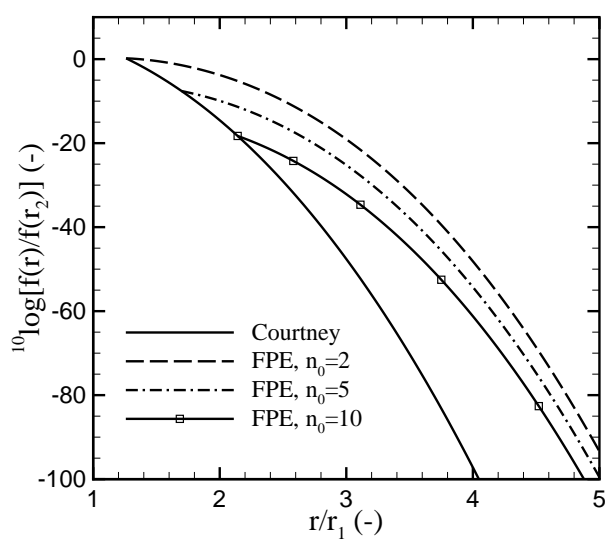

(a)

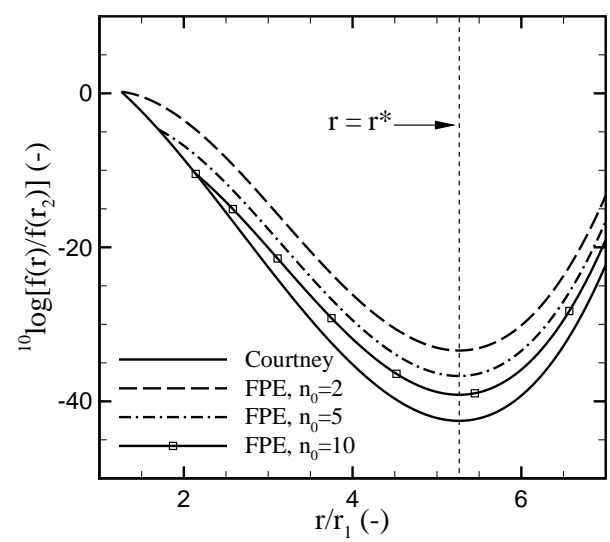

(b)

FIGURE 3.7: Equilibrium droplet size distributions for the KE (Courtney) and FPE, for water vapor at: (a) $T=253.2 \mathrm{~K}, S=0.5$; (b) $T=253.2 \mathrm{~K}, S=5.0$.

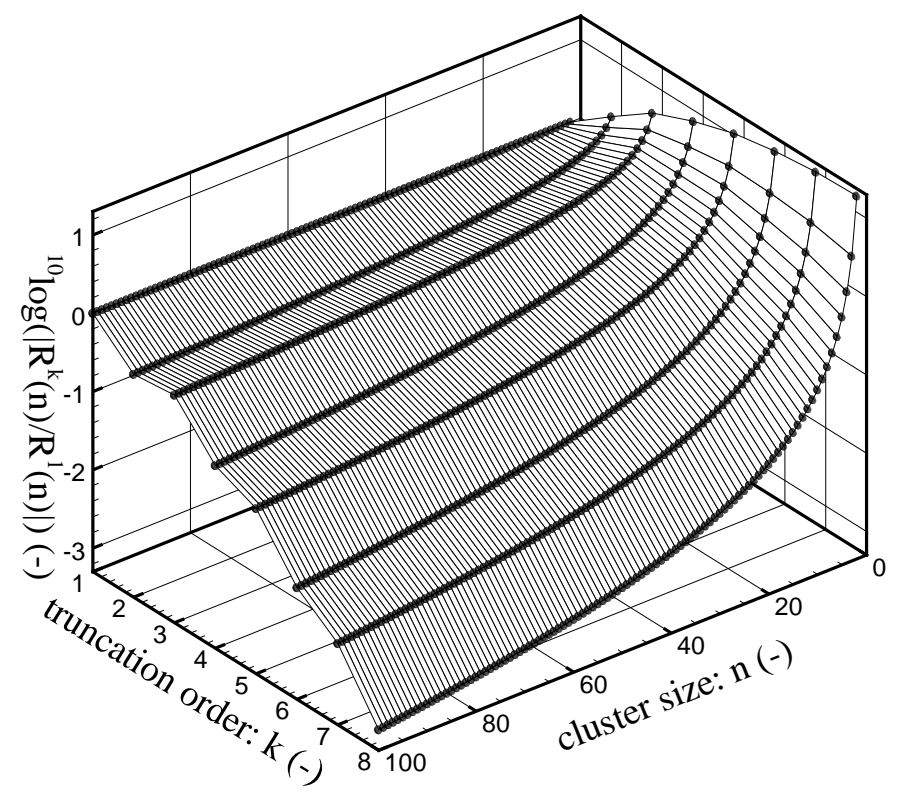

FIGURE 3.8: Truncation errors associated with the Courtney distribution for water vapor at $T=253.2 \mathrm{~K}$ and $S=0.5$.

accurately, even for very small droplets . There is, however, a noticeable difference in the magnitude of about $8 \%$ aft of the moving front. In this region, the difference 
between the GDE-solution and the KE-solution is less than 1\%, which is remarkable considering the fact that the former is merely a first-order approximation of the latter. In the region which immediately surrounds the moving front though, the FPE clearly performs better than the GDE, as the latter cannot capture the gradual transition of the size distribution to zero near the end of the front. In figures Figure 3.9.c and d the rear of the size distribution corresponding with the GDE abruptly stops, whereas the KE- and FPE-solutions gradually reduce to zero. This difference is a consequence of the denucleation boundary condition applied at $r_{0}=r\left(2 n^{*}\right)$, which removes all of the droplets of size $r<r_{0}$ in the GDE-solution. Overall, the solutions obtained with the various master equations show favorable agreement for this test case.

To explain this observation, the condensation fluxes $J_{n}$ and terms $T^{k}(n)$ of the Taylor series expansion in the generalized FPE (Eq. (3.16)) are examined. Using the KEsolution, the condensation fluxes $J_{n}$ (Eq. (3.2)) have been calculated and plotted in Figure 3.10, for the same four instants in time as in Figure 3.9. During the nucleation pulse ( $0 \leq t \leq 0.05 \mathrm{~ms}), J_{n}$ is independent of $n$ in the region aft of the front, meaning that the condition of steady state nucleation is satisfied: $d \hat{\rho}_{n} / d t=0$. Furthermore, $J_{n}=J_{C N T}$ in this region, which explains why the magnitude of the GDE-solution is very close to that of the KE-solution.

As the condition of steady state nucleation is satisfied, it now remains to be determined how important the second- and higher-order Taylor terms $T^{k}(n)$ are compared to the drift term $T^{1}(n)$. Figure 3.11.a and b depict the ratio $\left.T^{k}(n) / T^{1}(n)\right)$ for the times $t=0.025 \mathrm{~ms}$ and $t=0.17 \mathrm{~ms}$, respectively, for $k=2,3,4$. For $t=0.025 \mathrm{~ms}$ it is observed that the first- and second-order terms are of comparable magnitude, even for supercritical droplets, which seems to contradict the good agreement between the results of the GDE and the KE in this region. Furthermore, for very small droplets the third- and fourth-order terms are not small compared to the first- and second-order terms.

When the distribution evolves in time, the contribution of each Taylor term in the expansion of the condensation source term can be weighed against the difference in condensation fluxes $J_{n-1}-J_{n}$, in order to determine the significance of each term. During the pulse, however, droplets of size $n \leq 10^{4}$ are locally in a quasisteady state $\left(d \hat{\rho}_{n} / d t=0\right)$, which means that $J_{n-1}-J_{n}=0$. As all Taylor terms also tend to zero in this region, comparison of $T^{k}(n)$ with $T^{1}(n)$ is not a convenient method to reveal the importance of the higher-order terms. In this regard, it is more relevant to consider the fluxes $G^{k}(n)$ corresponding with each Taylor term $T^{k}(n)$, where:

$$
G^{k}(n) \equiv-\left.\frac{1}{k !} \frac{\partial^{k-1}}{\partial \zeta^{k-1}}\left[\left(f_{\zeta}(-1)^{k}+b_{\zeta}\right) \hat{\rho}(\zeta)\right]\right|_{\zeta=n} .
$$

From Eq. (3.17) and Eq. (3.62) it follows that $\frac{\partial G^{k}(n)}{\partial n}=-T^{k}(n)$. By setting $k=1$ in Eq. (3.62), the drift flux is obtained: $G^{1}(n)=\dot{n} \hat{\rho}(n)$, and for $k=2$ the diffusive flux: 


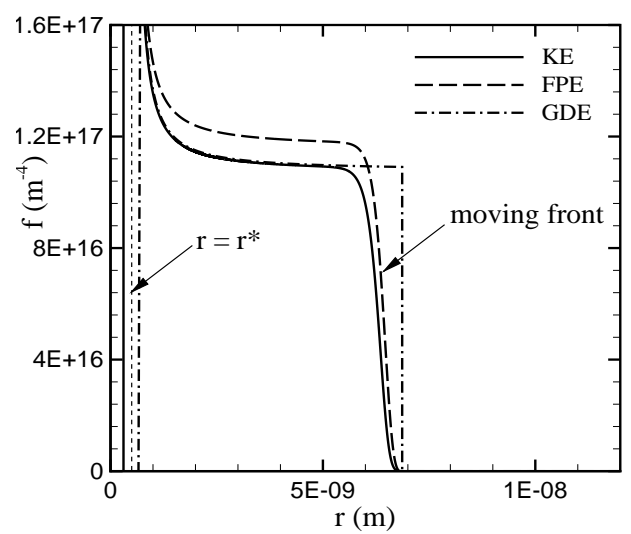

(a)

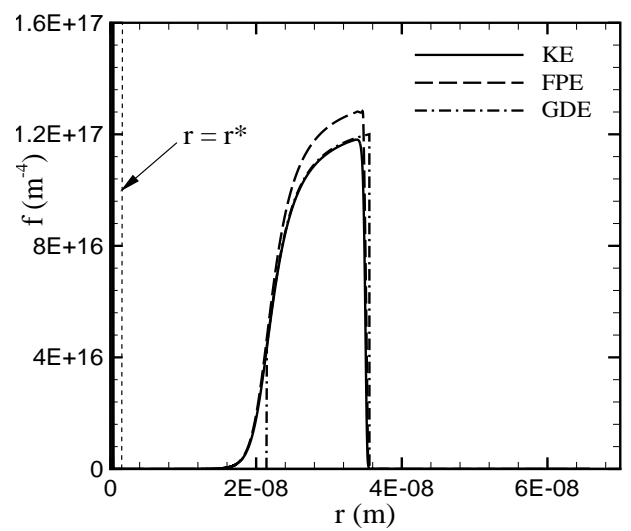

(c)

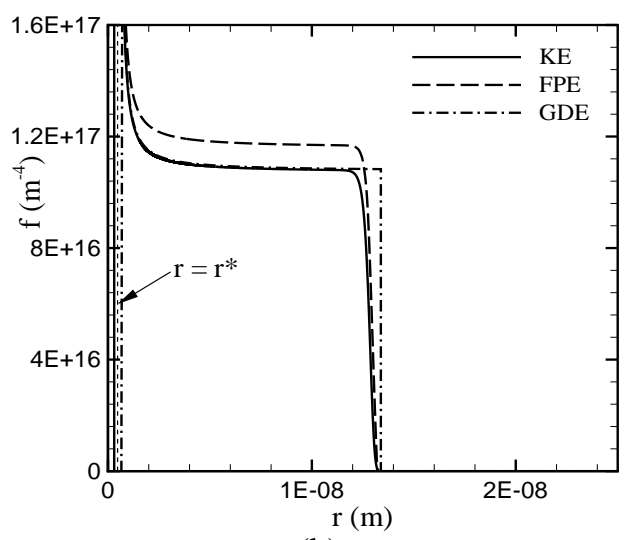

(b)

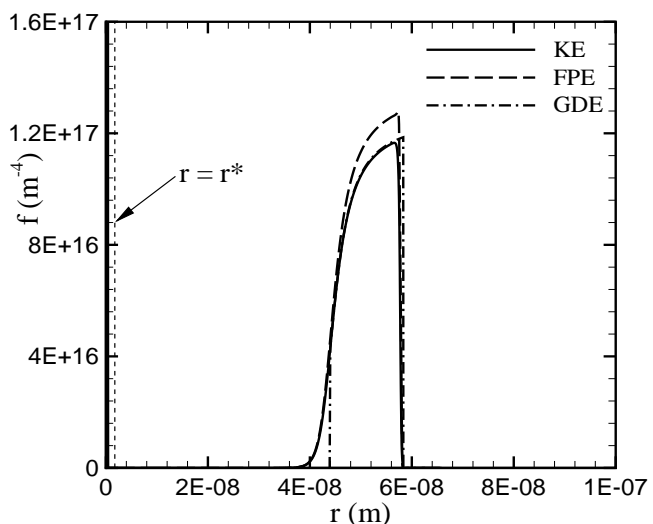

(d)

FIGURE 3.9: Droplet size distributions obtained with the KE, FPE, and GDE for the nucleation pulse experiment at four different times: (a) $t=0.025 \mathrm{~ms} ;(b) t=0.05 \mathrm{~ms}$ ; (c) $t=0.17 \mathrm{~ms} ;(d) t=0.29 \mathrm{~ms}$.

$G^{2}(n)=-\frac{\partial}{\partial n}(D \hat{\rho})(n)$. Equating the right-hand side of Eq. (3.3) and Eq. (3.16), the following relationship is obtained:

$$
\sum_{k=1}^{\infty} T^{k}(n)=\hat{J}(n-1)-\hat{J}(n),
$$

where the continuous function $\hat{J}(n)$ is equal to the discrete condensation flux $J_{n}$ for 


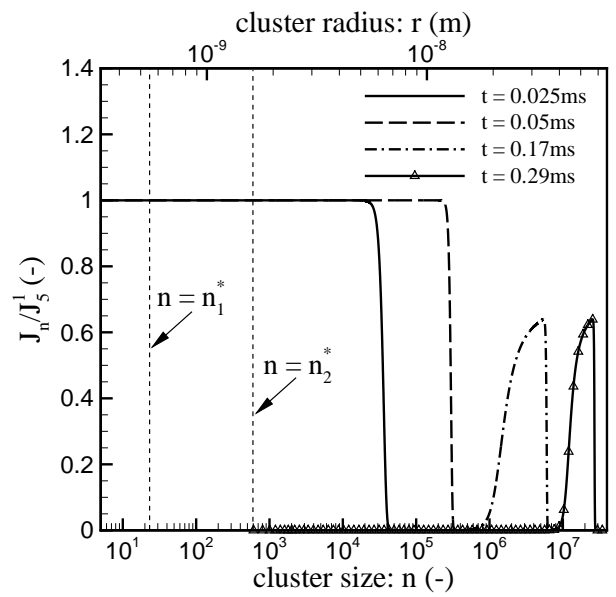

FIGURE 3.10: Evolution of condensation fluxes $J_{n}$ calculated by means of Eq. (3.2), based on the solution of the KE, using the Courtney equilibrium distribution Eq. (3.9) and with $\alpha_{n}=1$. The critical droplet sizes before and after the pulse are denoted by $n_{1}^{*}$ and $n_{2}^{*}$, respectively.

$n \in \mathbb{N}^{+}$. Integration of Eq. (3.63) from $n$ to $\infty$ then yields:

$$
\left[\sum_{k=1}^{\infty} G^{k}(\zeta)\right]_{n}^{\infty}=-\int_{n-1}^{n} \hat{J}(\zeta) d \zeta .
$$

As $\hat{\rho}=0$ for $n \rightarrow \infty$, and because $\hat{J}(n)=J$ is independent of $n$ in the quasisteady state, Eq. (3.64) may be simplified to:

$$
\sum_{k=1}^{\infty} \frac{G^{k}(n)}{J}=1 .
$$

Hence, for a quasisteady state solution, the importance of each Taylor term in the generalized FPE can be evaluated in an indirect manner by comparing the scaled fluxes $G^{k}(n) / J$ with each other. These fluxes are shown in Figures 3.11.c and d, for the times $t=0.025 \mathrm{~ms}$ and $t=0.17 \mathrm{~ms}$, respectively.

In Figure 3.11.c it is clearly observed that the drift flux $G^{1}(n)$ is the dominant term for supercritical droplets $\left(n>1.5 n^{*}\right)$, whereas below the critical size, the secondorder term becomes equally important. Figure 3.11.c actually confirms the validity of Muitjens' and Hill's suggestion to neglect the second-order term for $n>2 n^{*}$. This is underscored in Figure 3.11.e, which shows that $G^{1}\left(2 n^{*}\right) \approx 0.96 J$, and $G^{1}(n)+G^{2}(n) \approx$ $J$ for $n>n^{*}$. For very small subcritical droplets $\left(n<0.5 n^{*}\right)$, the contributions of the third- and fourth-order fluxes become significant, which relates to the inaccurate results of the FPE in this region. In retrospect, it is found that all necessary conditions 


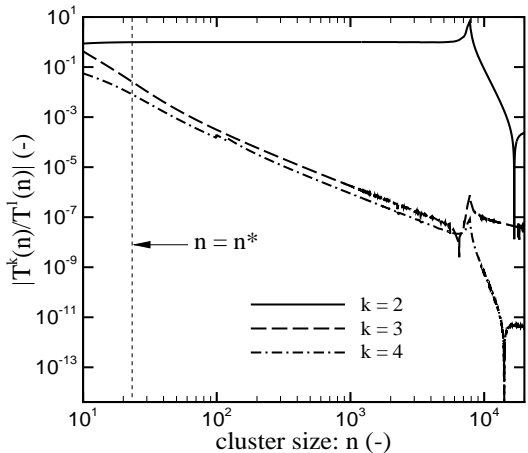

(a)

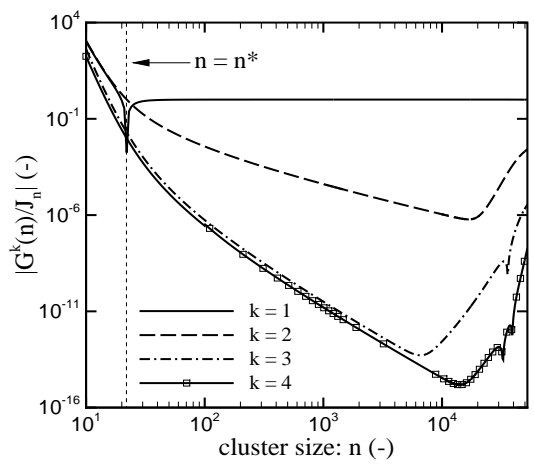

(c)

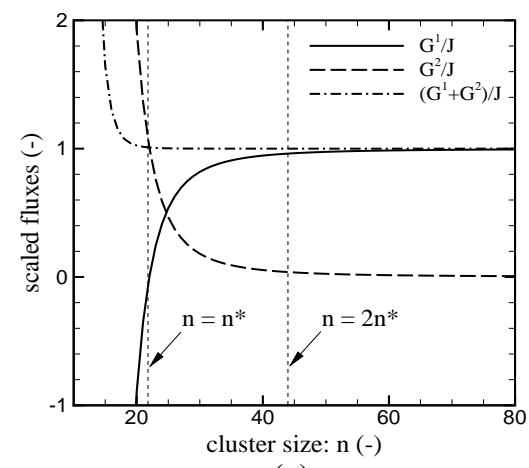

(e)

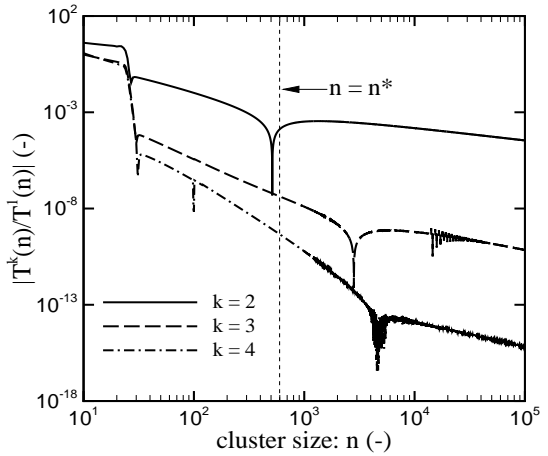

(b)

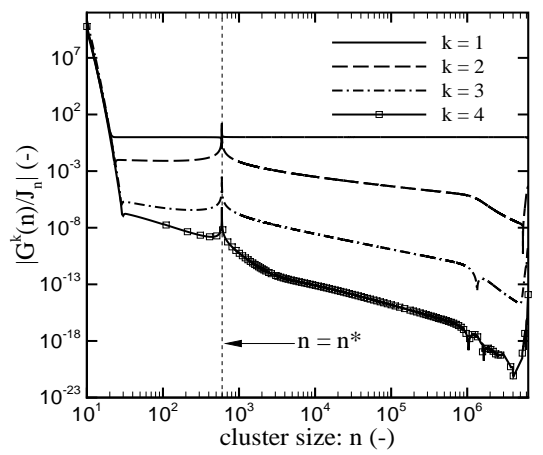

(d)

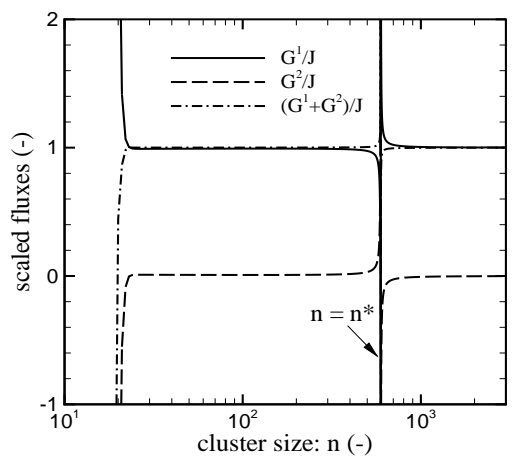

(f)

FIGURE 3.11: Second- $\left(T^{2}(n)\right)$, third- $\left(T^{3}(n)\right)$ and fourth-order $\left(T^{4}(n)\right)$ terms in the Taylor series expansion on the right-hand side of Eq. (3.16) at (a) $t=0.025 \mathrm{~ms}$ and (b) $t=0.17 \mathrm{~ms}$. The corresponding fluxes $G^{k}(n)$ are plotted in $(c)$ and $(d)$, respectively. The scaled first- and second-order terms and their sum are depicted in $(e)$ and $(f)$, for $t=0.025 \mathrm{~ms}$ and $t=0.17 \mathrm{~ms}$, respectively. 
for simplification of the KE to the GDE are satisfied during the nucleation pulse, which explains the good agreement between the predicted size distributions. For the period after the pulse $(t>0.05 \mathrm{~ms})$, the condensation fluxes vary with droplet size (Figure 3.10), so that the steady-state condition is no longer satisfied. The transient behavior of the size distribution only regards the large droplets $\left(n>n^{*}\right)$, for which both $T^{k}(n)$ (Figure 3.11.b) and $G^{k}(n)$ (Figure 3.11.d and f) show that the first-order term is dominant. Therefore, the GDE still is a valid approximation to the KE, even for slightly subcritical droplets $\left(n>0.5 n^{*}\right)$.

With respect to the FPE, it is observed in Figure 3.11 that incorporation of the second-order term should yield accurate results, even for droplets as small as $n=11$ in the period after the pulse. This is confirmed by Figure 3.12, where the FPEsolution is plotted for varying lower boundaries $n_{0}$. The best agreement is obtained with $n_{0}=15$; for the smaller values of $n_{0}=5$ and $n_{0}=10$, the small droplets tend to be near the equilibrium distributions corresponding with each master equation (Figure 3.12.c and d), which, as already seen in the previous discussion on the equilibrium distribution, leads to rather large differences between the KE- and FPE-solutions. This behavior characterizes the small droplets, and results in an overestimation of the liquid mass fraction at the onset of condensation when the FPE is employed. This is also the primary reason why it was not possible to obtain solution convergence for the FPE in the second test case.

\subsubsection{Test case 2: expanding nozzle flow}

Using the reconstructed nozzle profile in Figure 3.13.a, the FDE have been solved simultaneously with either the KE or the GDE. Figure 3.13.b shows the typical behavior of variables that characterize the flow field and the thermodynamic behavior of the system. Nucleation starts at $x \approx 0.02 m$, where the saturation reaches a maximum. This greatly enhances the formation of stable droplets, and causes the $\mathrm{D}_{2} \mathrm{O}$ liquid mass fraction $g$ to increase steadily. The latent heat release due to condensation results in a temperature rise, while simultaneously the flow is decelerated. The flow remains supersonic in the entire divergent section of the nozzle, and downstream of the nucleation point the vapor remains in a slightly supersaturated state.

To evaluate the influence of the higher-order derivatives in the master equation on the thermodynamic variables, the mixture temperature $T$ and $\mathrm{D}_{2} \mathrm{O}$ liquid mass fraction $g$ have been plotted for solutions obtained with the FDE/KE- and FDE/GDEsystems in Figures 3.14.a and b, along with the laser absorbtion measurement (LAM) data from [119]. The nucleation rate in the GDE has been obtained by using either the CNT-model, the CNT-model with the empirical Wölk and Strey-correction, and finally, the ICCT-model (see appendix B and Eq. (3.12)). The predicted temperature and liquid mass fraction profiles obtained with the KE and GDE-CNT models are very close to each other, but they differ significantly from the measured data. It is observed that both models predict the onset of condensation too late and that the pre- 


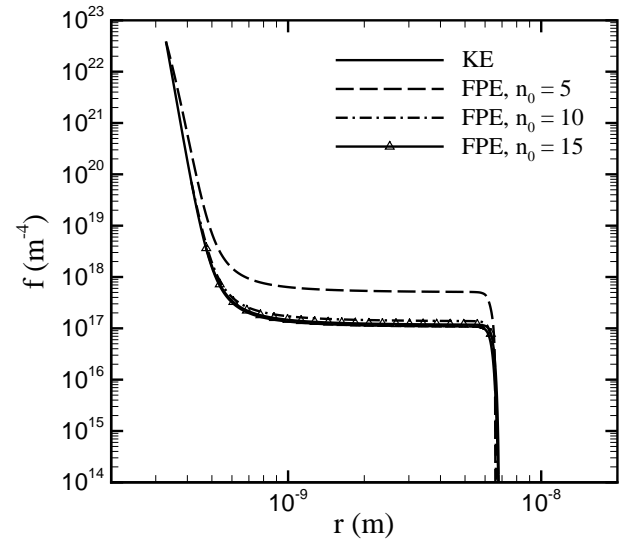

(a)

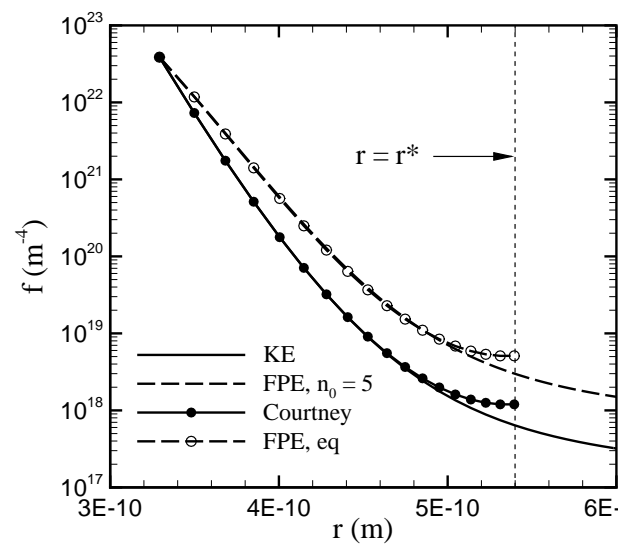

(c)

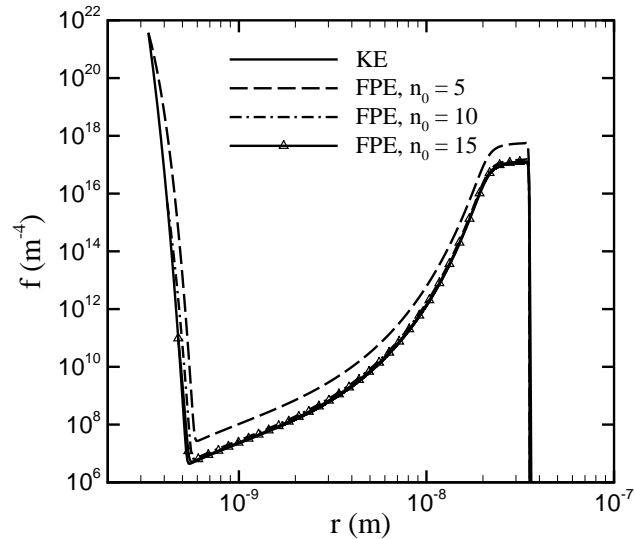

(b)

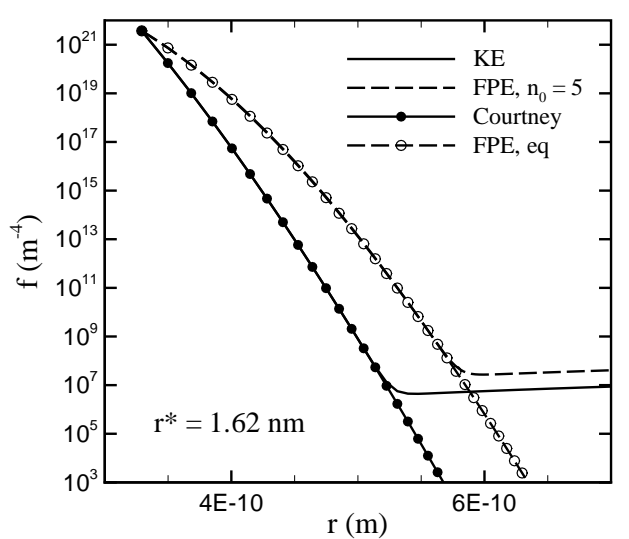

(d)

FIGURE 3.12: Solution of the FPE for various lower boundary positions: $n_{0}=5,10$ and 15, at two times: (a) $t=0.025 \mathrm{~ms} ;(b) t=0.17 \mathrm{~ms}$. The solid line is the solution of the KE using the Courtney equilibrium distribution with $\alpha_{n}=1$. Figures $(c)$ and $(d)$ are close-ups of respectively $(a)$ and $(b)$, depicting the equilibrium distributions for the KE (Courtney) and the FPE $\left(n_{0}=5\right)$.

dicted temperature is too high in the region downstream of the nucleation zone. The GDE employing the CNT-model with the Wölk and Strey-correction does a better job of capturing the onset-point, but the temperature downstream of the nucleation zone is again overestimated. Finally, the GDE with the ICCT-model is seen to predict the onset of condensation much too early, but it does yield a final temperature which is closer to the measurement data.

The droplet size distributions obtained by solving the FDE/KE-, or the FDE/GDE- 


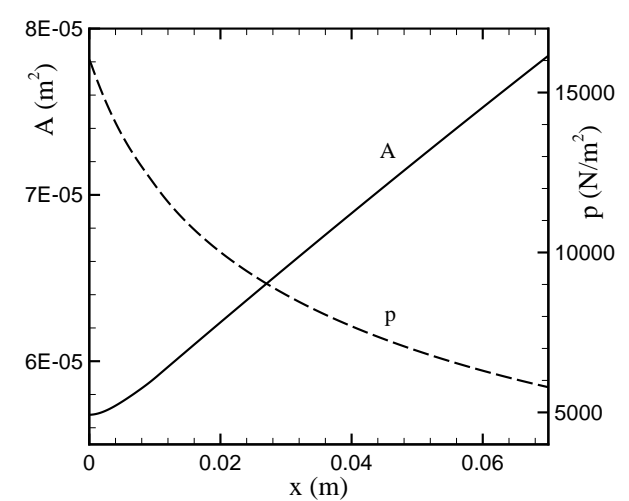

(a)

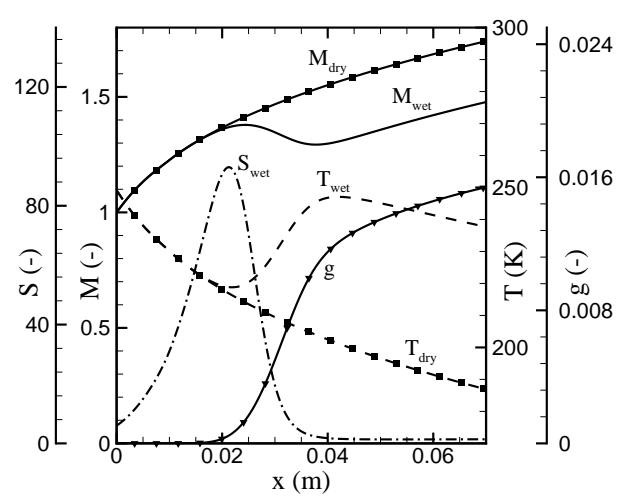

(b)

FIGURE 3.13: (a) Nozzle cross-sectional area and dry-flow pressure profile used in the second test case; (b) solution for the Mach number $M$, temperature $T, \mathrm{D}_{2} \mathrm{O}$ liquid mass fraction $g$ and saturation $S$ along the nozzle axis. The throat of the nozzle is located at $x=0.0 \mathrm{~m}$.

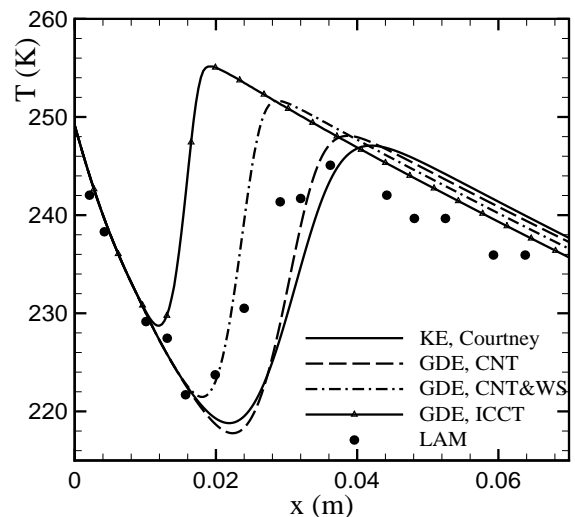

(a)

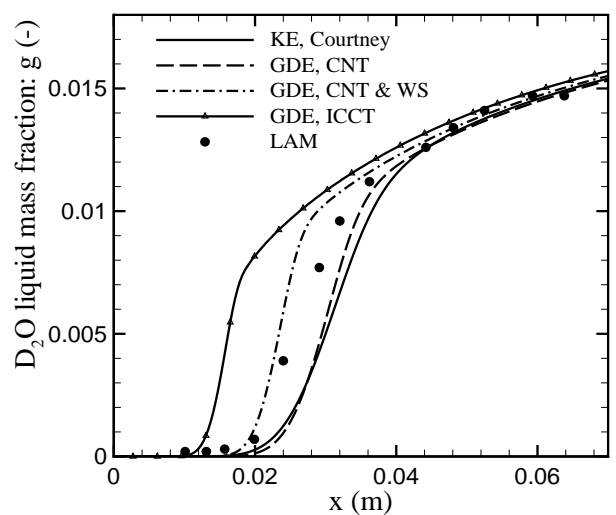

(b)

FIGURE 3.14: Comparison of temperatures $\mathrm{T}$ and $\mathrm{D}_{2} \mathrm{O}$ liquid mass fractions $\mathrm{g}$ for the expanding nozzle flow obtained from various simulations and from experiment.

system with various $n_{0}$ are shown in Figures 3.15.a-c, with close-ups of the nucleation zone in the $r-x$ plane in Figures 3.16.a-c. Figures 3.17.a-d show the same droplet size distributions at four positions along the nozzle axis. From the KE-solution in Figures 3.15.a, 3.16.a and 3.17, it is observed that the distribution becomes bi-modal in the nucleation zone, with an exponentially decreasing tail in the region of small droplets $(r<0.5 \mathrm{~nm}$, see Figure 3.17.b). Of special interest is 


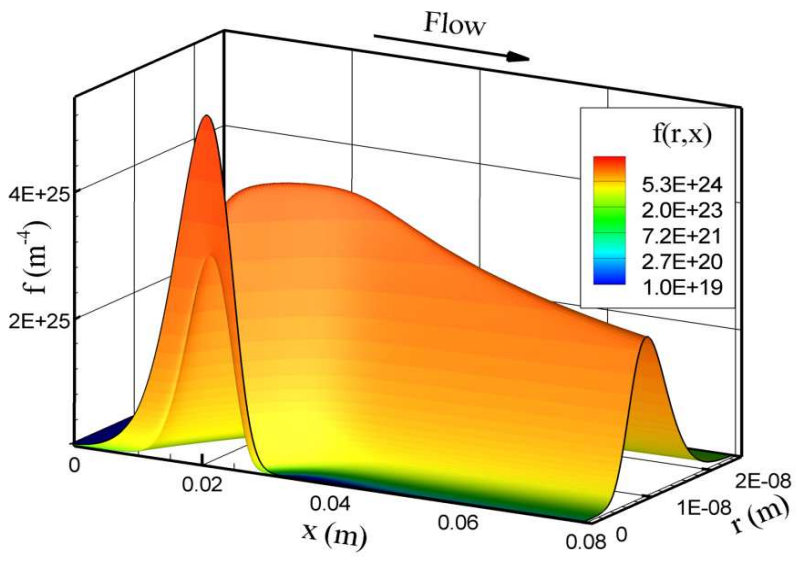

(a)

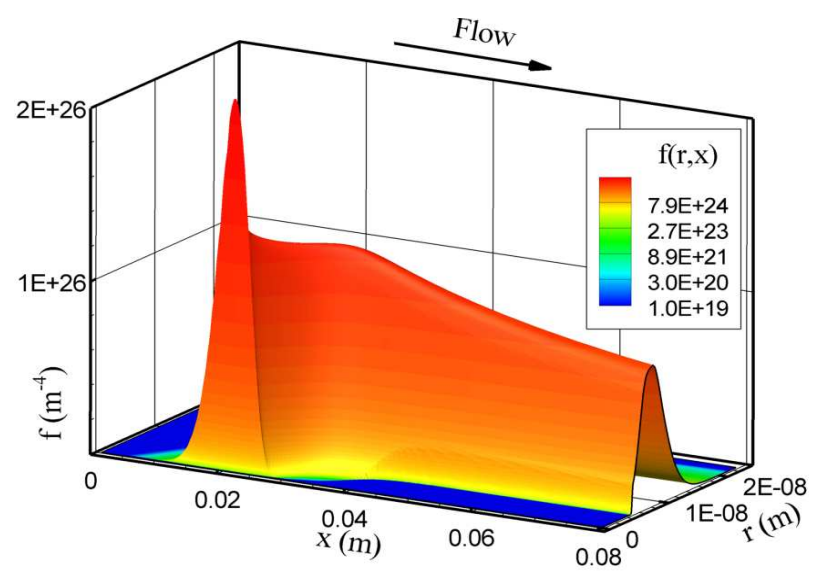

(b)

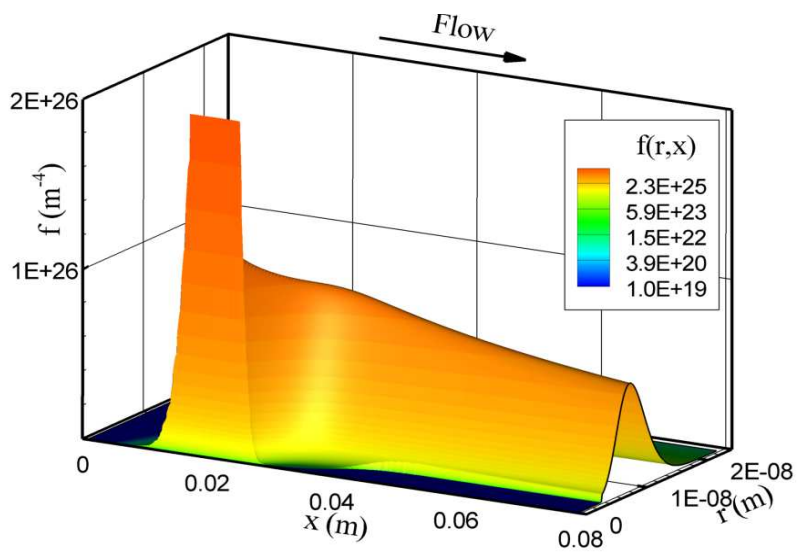

(c)

FIGURE 3.15: Solution for the droplet radius distribution function along the nozzle axis: (a) $K E(n \geq 5)$; (b) GDE with $n_{0}=2 n^{*}$; (c) GDE with $n_{0}=1.1 n^{*}$ (the plot has been truncated to match the scale of the size distribution in $(b))$. 


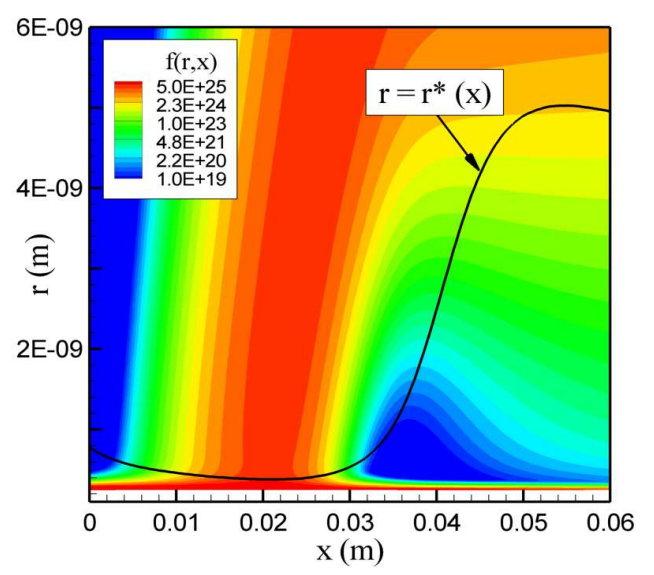

(a)

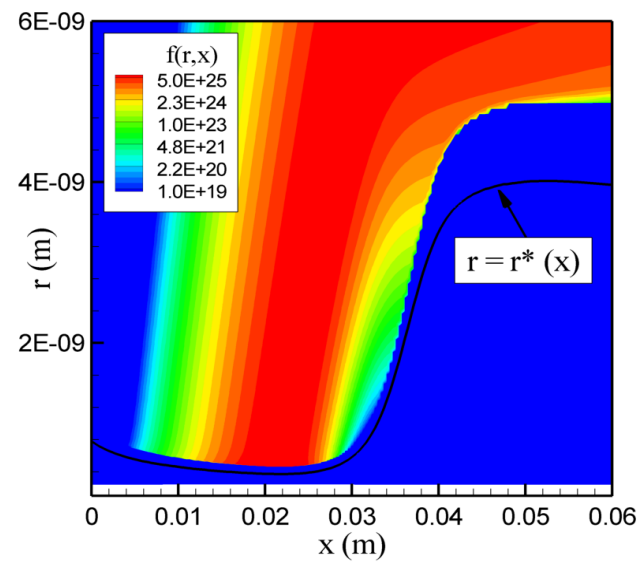

(b)

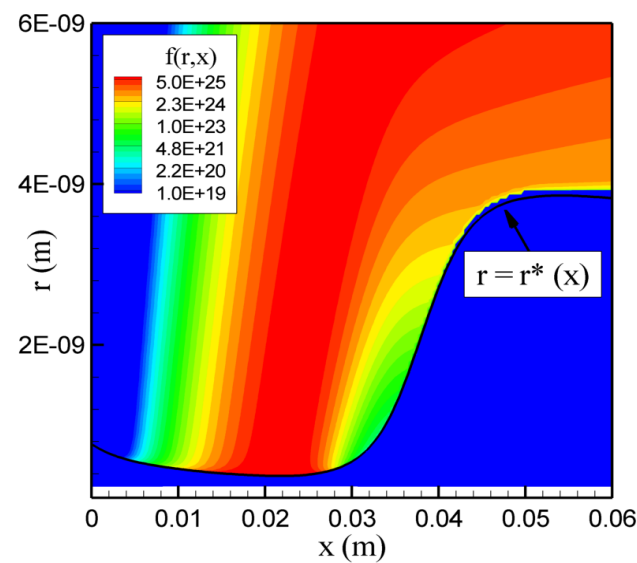

(c)

Figure 3.16: Close-up of the droplet radius distribution function within the nucleation zone: (a) KE ; (b) GDE with $n_{0}=2 n^{*}$; (c) GDE with $n_{0}=1.1 n^{*}$ 


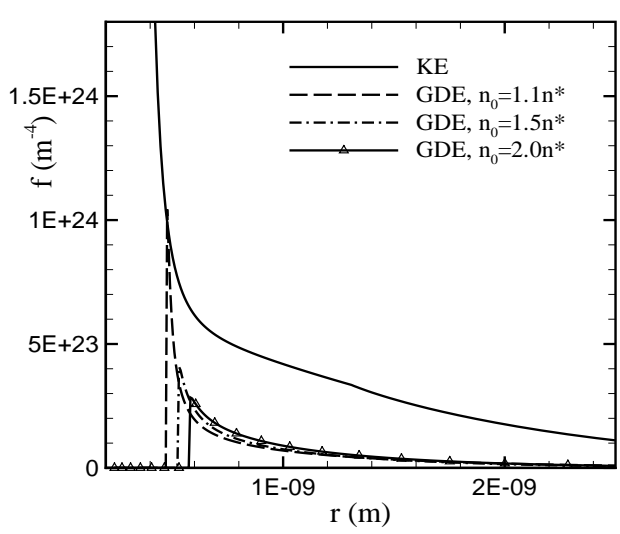

(a)

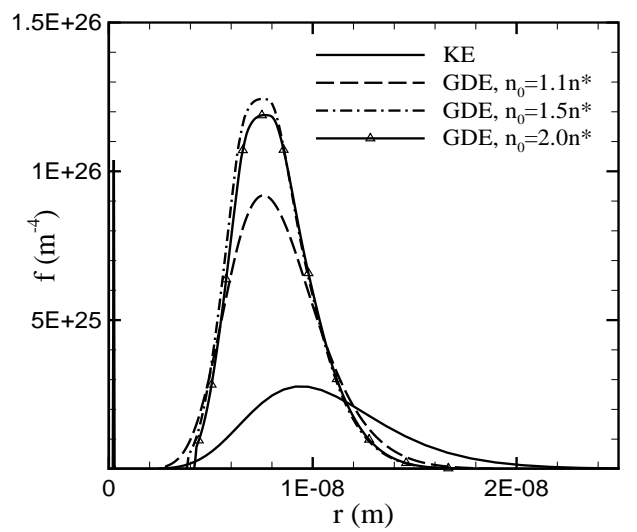

(c)

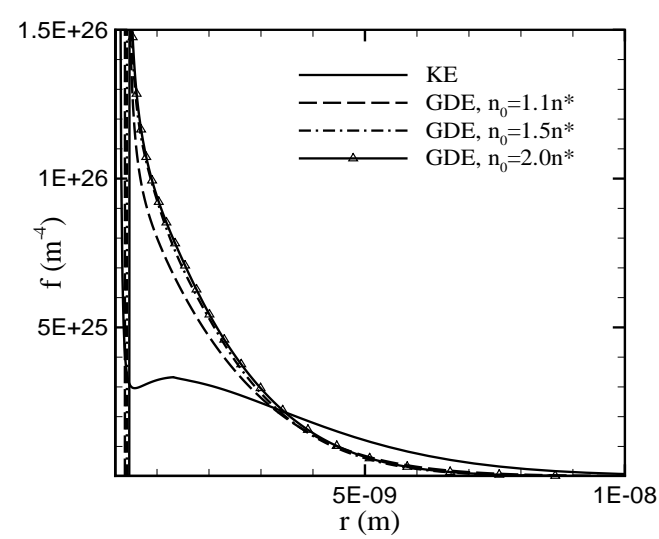

(b)

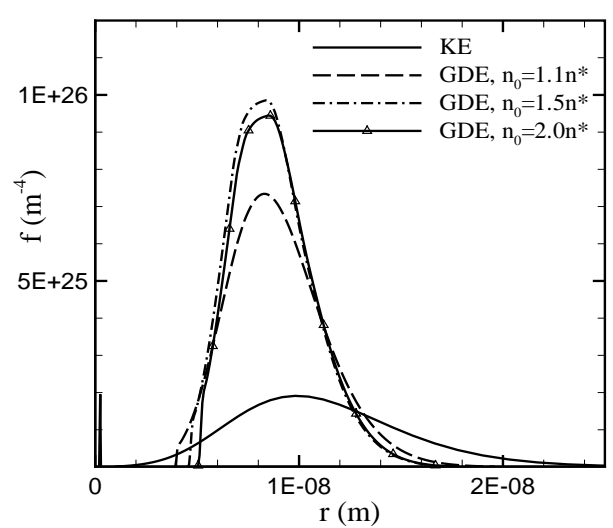

(d)

FIGURE 3.17: Comparison of droplet radius distributions at four different positions along the nozzle: (a) $x=0.01 \mathrm{~m} ;$; (b) $x=0.02 \mathrm{~m} ;$; c $) x=0.04 \mathrm{~m}$; (d) $x=0.06 \mathrm{~m}$. The $K E$ has been solved by using the Courtney equilibrium distribution with $\alpha_{n}=1$. The $G D E$ has been solved on various domains, with the minimum droplet size $n_{0}$ varied between the values $1.1 n^{*}, 1.5 n^{*}$, and $2 n^{*}$. The nucleation rate is calculated by means of the CNT-expression.

the region in the vicinity of the critical droplet size $r^{*}$, located within the nucleation zone. As shown in Figure 3.16.a, the solution shows strong gradients within this zone, and in the immediate vicinity of the critical line, in the region where $r^{*}$ rises, the distribution attains very small values, which implies that there is almost a void of droplets in this area. This behavior was also reported in earlier work [107], in which the GDE was solved with $n_{0}=2$, and with the nucleation source term active 
at $n=n^{*}$. The GDE-solutions given in Figures 3.16.b and c show a void of droplets below $r=1.26 r^{*}$ and $r=1.03 r^{*}$, respectively, in accordance with the denucleation condition at the lower boundaries $n_{0}=2 n^{*}$ and $n_{0}=1.1 n^{*}$, respectively. For the KE, this region is further characterized by nett evaporation, as the curve corresponding with the position $x=0.03 \mathrm{~m}$ in Figure 3.18 .a (see the insert) shows slightly negative fluxes for the small droplets.

When comparing the various GDE-solutions with the KE-solution in Figures 3.15, (3.16) and (3.17), it is observed that at the onset of condensation (Figure 3.17.a), the distributions have a similar shape, but different magnitude. At the point where maximum supersaturation is achieved ( $x \approx 0.02 m$, Figure 3.17.b), the GDE-solutions continue to display an exponentially decreasing trend, whereas the KE-distribution shows both a local minimum and a maximum. Further downstream of the nucleation zone (Figure 3.17.c and d), the extrema in the KE-solution become more pronounced and there is a clear separation between the size distribution for the small droplets and that for the large droplets. In this region, each GDE-solution has a maximum, which is much larger in magnitude compared to that of the KE-solution. The discontinuity on the left side of the GDE-distributions (see Figure 3.17.d) is a consequence of denucleation and is most pronounced for the solution with $n_{0}=2 n^{*}$. Furthermore, each of the GDE-distributions is shifted towards the smaller droplets compared to the solution of the KE. It is this shift which compensates for the difference in magnitude, so that the liquid mass fractions corresponding with the GDE-solutions and the KE-solution (see Figure 3.14.b) differ only slightly.

Having observed the rather large differences between the size distributions obtained with the KE and GDE, it is concluded that the conditions which allow for simplification of the KE to the GDE are not satisfied. The first condition to be examined concerns the quasisteady state behavior of the size distribution. To relate the behavior of the size distribution in the nozzle flow to the observations from the nucleation pulse experiment, it is convenient to rewrite the KE in Eq. (3.3) in the following Lagrangian form

$$
\frac{D \check{\rho}_{n}}{D t}=\check{J}_{n-1}-\breve{J}_{n}, \quad n=2,3, \ldots,
$$

where: $\breve{\rho}_{n} \equiv \hat{\rho}_{n} / \rho$, and: $\breve{J}_{n} \equiv J_{n} / \rho$. Based on Eq. (3.66), the steady state nucleation is obtained when $\breve{J}_{n}$ is independent of $n$, for droplets in the vicinity of $n^{*}$. Considering Figure 3.18.a, it is clear that the steady state condition is not satisfied within the nucleation zone. This is also reflected in Figure 3.18.b, which shows that $J_{C N T}$ differs by an order of magnitude from the condensation flux $J_{n^{*}}$ calculated at $n=n^{*}$. Having determined that the steady state condition is not satisfied, the next step is to evaluate the importance of the second and higher-order terms $T^{k}(n)$ in the Taylor series expansion of Eq. (3.16) relative to the first-order term $T^{1}(n)$. Figures 3.19.a-d show the changes in the scaled Taylor terms $T^{k}(n)$ for $k=2,3,4$, along with the location of the critical droplet size. The curves in Figures 3.19.a, b and c are associated with 
points within the nucleation zone, and here it is observed that the second-order term remains important in the vicinity of the critical droplet size, whereas the third- and fourth-order terms are only important for very small subcritical droplets. Clearly, the generation of new droplets at $n_{0}=2 n^{*}$, as suggested by Muitjens and Hill, is not valid in this particular case, as the second-order term is of the same order of magnitude as the drift term. The curves in Figure 3.19.d correspond to a position far downstream of the nucleation zone, where it is observed that the higher-order terms are relatively insignificant, even for subcritical droplets as small as $n=0.01 n^{*}$. Apparently, the evolution of the droplet size distribution in this region may be described by droplet growth alone, which makes the GDE a valid approximation downstream of the nucleation zone.

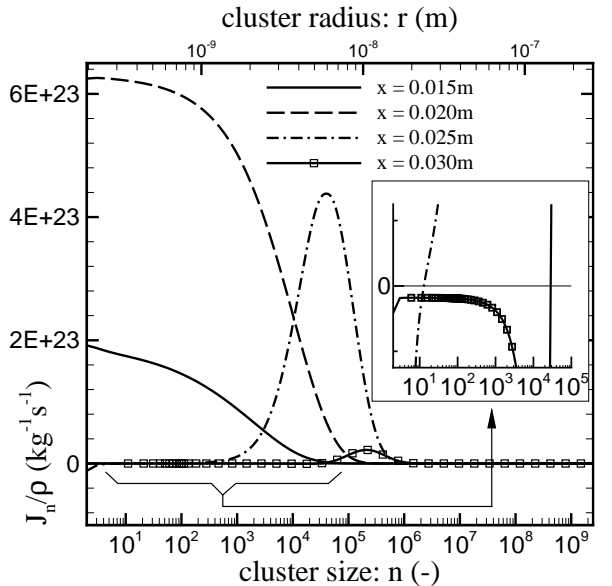

(a)

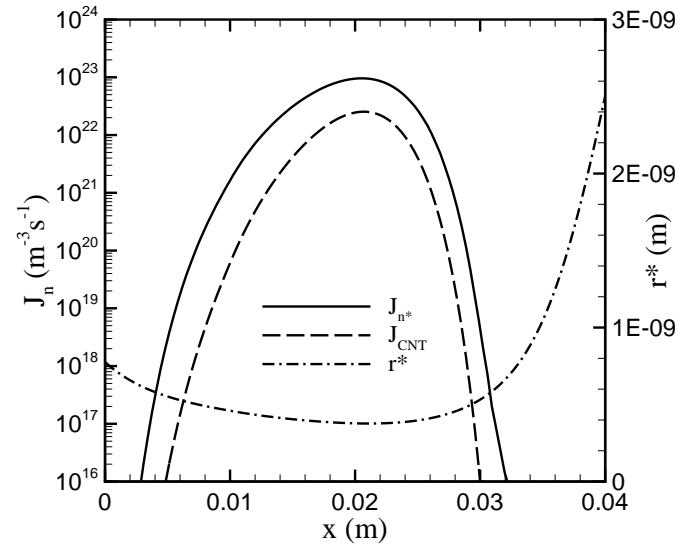

(b)

FIGURE 3.18: (a) Condensation fluxes calculated by means of Eq. (3.2), based on the solution of the KE, employing the Courtney equilibrium distribution $\left(\alpha_{n}=1\right)$; the insert shows a close-up for the small droplets. (b) Comparison between the critical condensation flux $J_{n^{*}}$ and the CNT-nucleation rate $J_{C N T}$.

It is noted that in previous work [46], [133], the boundary $n_{0}$ has been placed at droplets larger than dimers $(n>2)$, with the number density at the boundary specified by the equilibrium distribution: $\hat{\rho}_{n_{0}}=\hat{\rho}_{n_{0}}^{e q}$. As shown in Figures 3.20.a-d, this assumption appears to be valid, as long as the lower boundary is taken sufficiently small. Obviously, $n_{0}$ should be taken smaller than $n^{*}$, as the equilibrium distribution shows an exponentially increasing (and thus non-physical) trend for $n>n^{*}$.

From Figure 3.18.a, it is seen that $J_{n} \neq 0$ for the small droplets, whereas in the case of the equilibrium distribution, the condensation fluxes are exactly zero. Using Eq. (3.2) and Eq. (3.6), the ratio between successive number densities may be expressed 


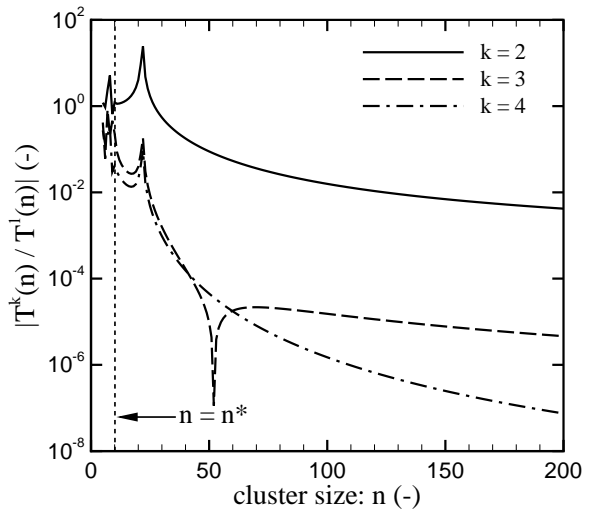

(a)

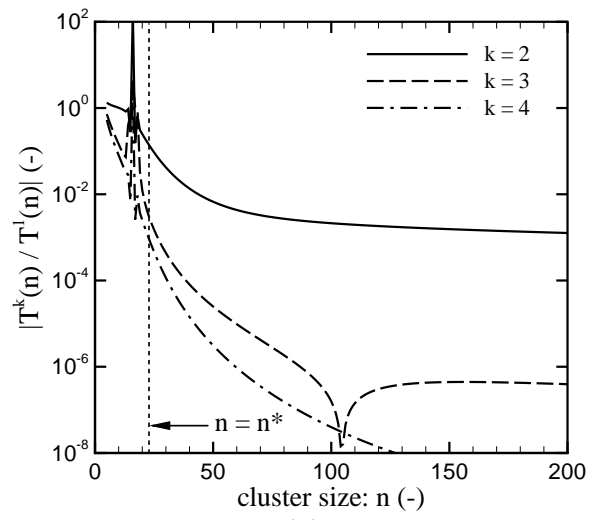

(c)

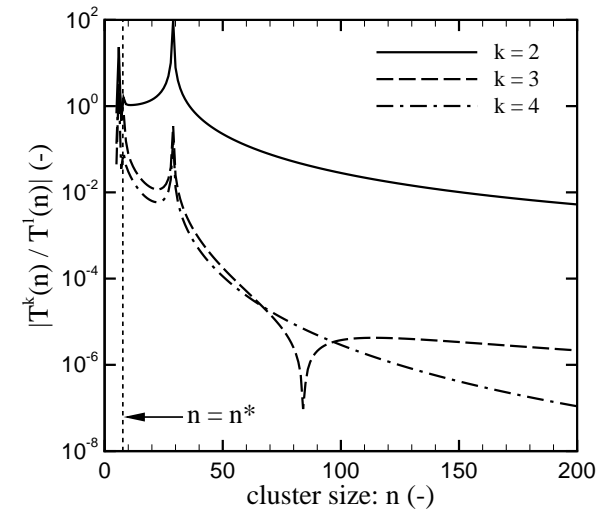

(b)

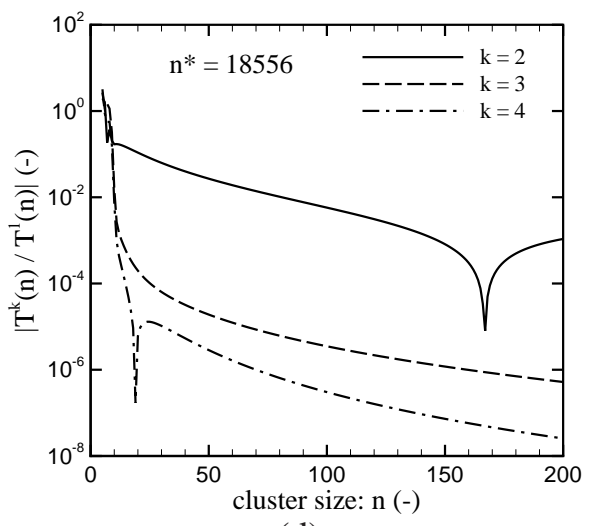

(d)

FIGURE 3.19: Second- $\left(T^{2}(n)\right)$, third- $\left(T^{3}(n)\right)$ and fourth-order $\left(T^{4}(n)\right)$ terms in the Taylor series expansion on the right-hand side of Eq. (3.16). All terms have been scaled with the first-order term $T^{1}(n)$. The dashed vertical line indicates the critical droplet size $n^{*}$; the graphs correspond with the following positions along the nozzle: (a) $x=0.015 m$; (b) $x=0.02 m$; (c) $x=0.03 m$; (d) $x=0.06 m$. 


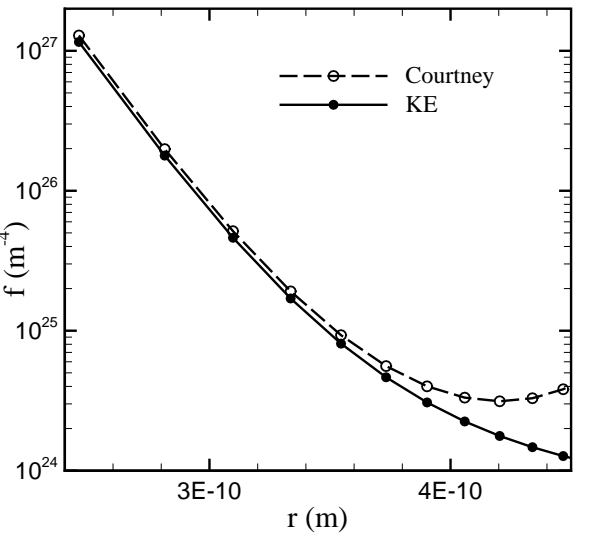

(a)

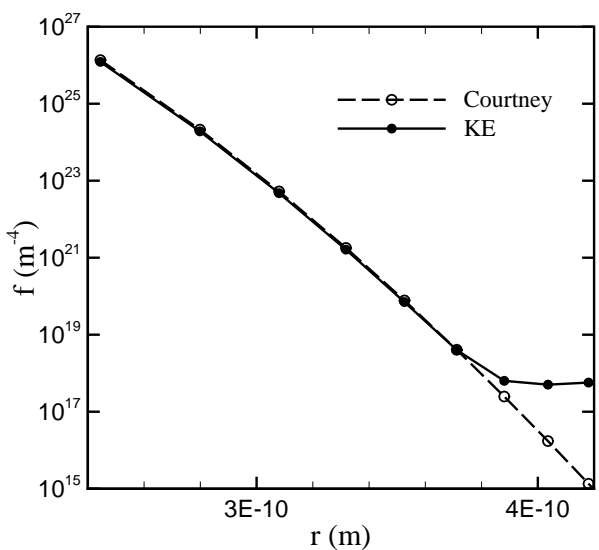

(c)

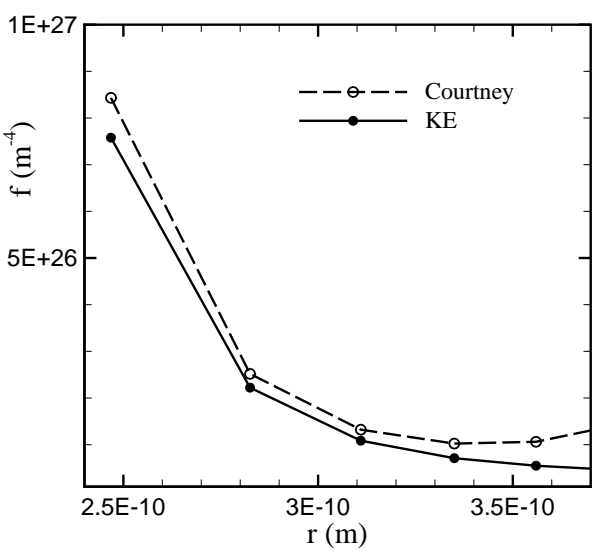

(b)

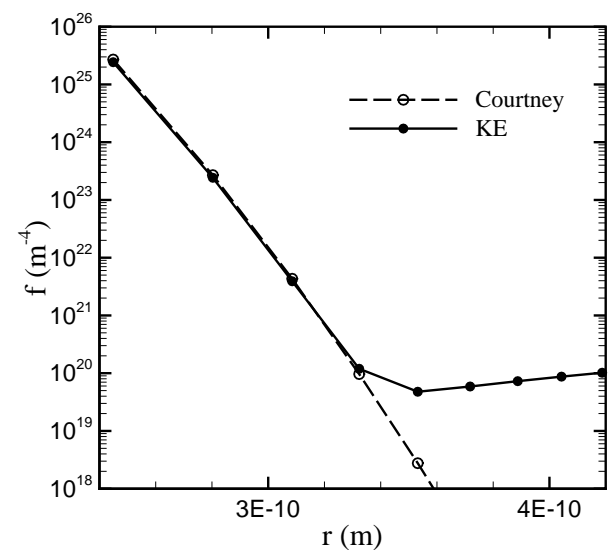

(d)

FigURE 3.20: Close-up of droplet radius distributions obtained by means of the KE, along with the Courtney equilibrium distribution at four different positions along the nozzle: (a) $x=0.01 \mathrm{~m}$; (b) $x=0.02 \mathrm{~m}$; (c) $x=0.04 m$; (d) $x=0.06 \mathrm{~m}$. 


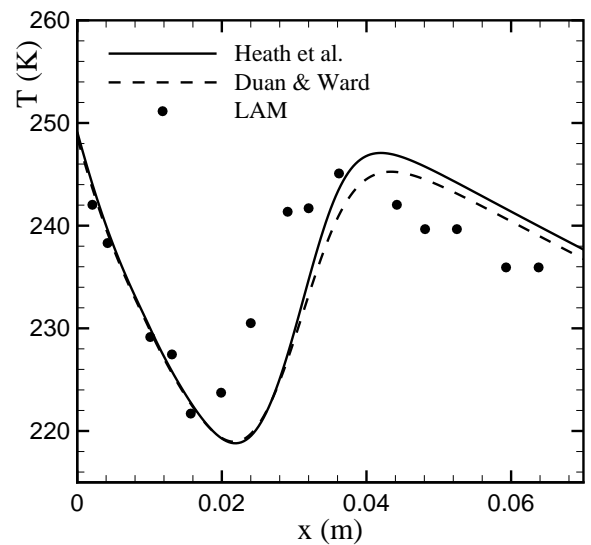

(a)

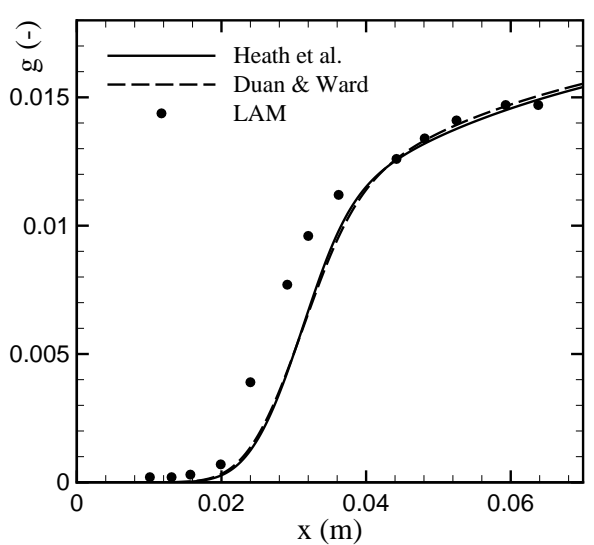

(b)

FIGURE 3.21: Comparison of temperature and $\mathrm{D}_{2} \mathrm{O}$ liquid mass fraction profiles for the surface tension expressions taken from [44] and [28]. The KE has been solved using the Courtney equilibrium distribution for the backward rate $\left(\alpha_{n}=1\right)$.

as:

$$
\frac{\hat{\rho}_{n+1}}{\hat{\rho}_{n}}=\frac{\hat{\rho}_{n+1}^{e q}}{\hat{\rho}_{n}^{e q}}\left[1-\frac{J_{n}}{f_{n} \hat{\rho}_{n}}\right] .
$$

Due to the high concentration of small droplets, it appears that $\frac{J_{n}}{f_{n} \hat{\rho}_{n}} \ll 1$ along the entire nozzle, which is consistent with the observation that the size distribution is almost equal to the equilibrium size distribution for these small droplets.

\subsubsection{Sensitivity analysis for the Kinetic Equation}

\section{Uncertainty in the surface tension}

As the equilibrium distributions depend exponentially on the surface tension $\sigma$, it is useful to analyze the sensitivity to uncertainties in the surface tension model. The slightly different $\mathrm{D}_{2} \mathrm{O}$ surface tension models reported in [44] and [28] lead to the temperature and liquid mass fraction profiles shown in Figure 3.21.a and b, when employed in the KE. It appears that the temperature is slightly sensitive to the surface tension model, whereas the liquid mass fraction is nearly unaffected.

\section{Uncertainty in the sticking probability}

To investigate the sensitivity with respect to the sticking probability, the temperature and liquid mass fraction profiles obtained with the various model expressions for $\alpha_{n}$ in the KE are shown in Figure 3.22.a and b. It is observed that all other models lead to some degree of condensation-onset delay, when compared to $\alpha_{n}=1$ (curve (i)). 
Downstream of the nucleation zone, however, all predicted temperatures and liquid mass fractions coincide, with the temperature being overestimated by all models.

\section{Uncertainty in the equilibrium size distribution}

The four equilibrium distributions (viz. Eq. (3.9), (3.10), (3.11), and (3.15)) which are used to model the backward rates, with $\alpha_{n}=1$ for all $n$ and employing the $\mathrm{KE} / \mathrm{FDE}$ model, lead to the temperature and liquid mass fraction profiles shown in Figures 3.22.a and b. The variation of the equilibrium model clearly has a pronounced influence on both variables, although the Kelvin- and SCC equilibrium distributions differ only slightly.

The Wölk and Strey-corrected Courtney distribution, which was obtained from nucleation pulse experiments, yields the best prediction of condensation onset in the nozzle flow when compared to the experiment. In contrast, the SCC and Kelvin models predict the onset much too early.

At the onset of condensation the distribution is close to equilibrium, and the liquid mass fraction is predominantly determined by the relatively high number densities associated with the small droplets. The accurate modelling of the equilibrium distribution for droplets as small as dimers is an active field of research, where advanced simulation methods are used to determine a.o. the Gibbs free energy for very small droplets [77].

Regardless of the observed differences in the nucleation zone, all models overestimate the temperature level in the region downstream of the nucleation zone.

\subsection{Conclusions}

The kinetic equation (KE), and its first- and second-order approximations, the general dynamic equation (GDE) and the Fokker-Planck equation (FPE), respectively, have been evaluated based on (a) their equilibrium distributions, (b) a nucleation pulse experiment, and (c) an expanding nozzle flow. The backward rates are derived from the forward rates using four different equilibrium distributions. Three of these distributions were taken from the literature, the fourth is proposed in the present paper and consists of a Wölk and Strey correction of the Courtney distribution.

The equilibrium distribution of the FPE reveals large differences when compared to the KE equilibrium distribution, whereas the GDE does not have an equilibrium distribution at all. Both observations are directly related to the lack of higher-order terms in the FPE and the GDE.

For the nucleation pulse experiment, good agreement is observed between all three models in the region of large droplets. Furthermore, the FPE also appears to be accurate for smaller droplets. These observations are a direct consequence of the fact 


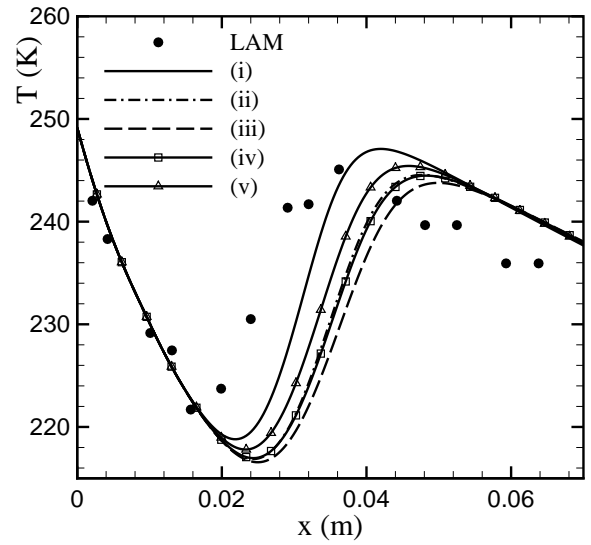

(a)

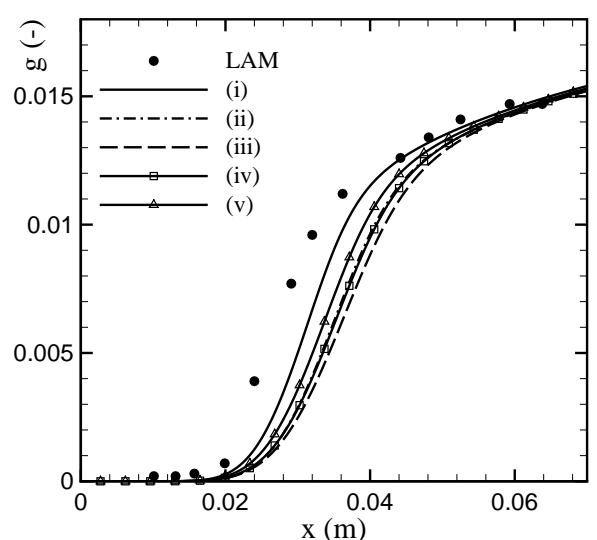

(b)

FiguRe 3.22: Comparison of temperature and $\mathrm{D}_{2} \mathrm{O}$ liquid mass fraction profiles for various sticking probabilities $\alpha_{n}$ : (i) $\alpha_{n}=1$; (ii) $\alpha_{n}=0.8$ (iii) $\alpha_{1}=10^{-2}, \alpha_{\infty}=1$, $n_{\infty}=10$; (iv) $\alpha_{1}=10^{-1}, \alpha_{\infty}=1, n_{\infty}=10$; (v) $\alpha_{1}=10^{-2}, \alpha_{\infty}=1, n_{\infty}=5$. The size distribution is obtained by solving the KE using the Courtney equilibrium distribution.

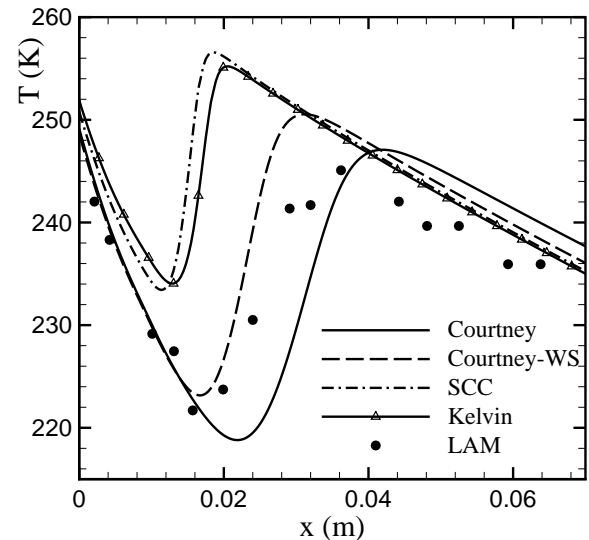

(a)

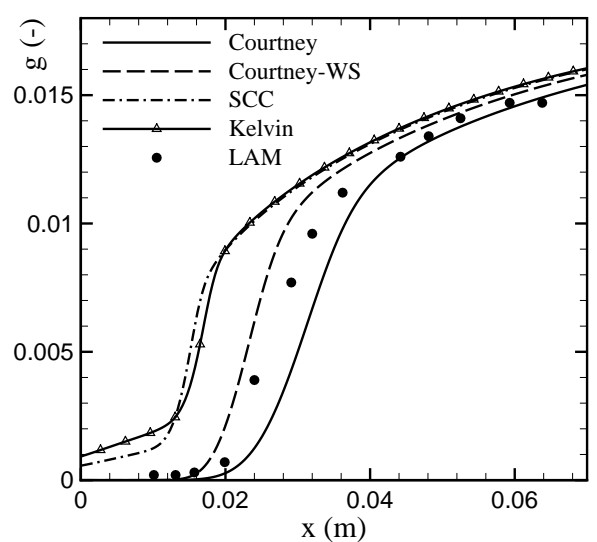

(b)

FIGURE 3.23: Comparison of temperature and $\mathrm{D}_{2} \mathrm{O}$ liquid mass fraction profiles for various equilibrium size distributions: Courtney, SCC, Kelvin and Courtney with the Wölk and Strey-correction. The sticking probability is set to unity: $\alpha_{n}=1$. 
that a quasisteady state is achieved during the nucleation pulse.

For the expanding nozzle flow, it appeared impossible to obtain a converged solution with the FPE model due to the unrealistic equilibrium distribution of the FPE. The GDE and KE solutions that were obtained from two-way coupled simulations, have been compared to data from a physical experiment. The size distributions obtained with the two models are very different, whereas the predicted temperatures and liquid mass fractions show good agreement when the KE is solved using the Courtney equilibrium distribution in the backward rates, and the GDE is solved using the classical nucleation theory. Both models produce solutions that differ considerably from the experimental data.

A sensitivity study of the KE-solution with respect to uncertainties in (a) the surface tension model, (b) the sticking probability, and (c) the equilibrium distribution (to model backward rates), revealed that both the sticking probability and the equilibrium distribution have a significant influence on the predicted condensation onset. The proposed Wölk and Strey-corrected Courtney distribution yields the best agreement with the experimental data. All four equilibrium models lead to an overestimated temperature downstream of the condensation onset point, which may be due to non-isothermal condensation. 


\section{REDUCED MODELS FOR SINGLE COMPONENT CONDENSATION}

In this chapter an evaluation is made of reduced models which generate approximate solutions for condensing flows. The models utilize the method of moments (MOM), for which two variants are considered: the MOM derived from the kinetic equation $(K E)$, and its large droplet-approximation derived from the general dynamic equation (GDE). Two well-known closure methods for the MOM, i.e., closure based on mean droplet size-approximations, and quadrature approximations, are qualitatively evaluated, based on key requirements that have been identified from steady-state and transient characteristics of the KE. It is found that neither of the closure methods performs satisfactorily, and that the quadrature approximations severely suffer from a lack of robustness. Finally, a numerical simulation of a condensing nozzle flow is utilized to quantify the influence of the closure error in the MOM. For the particular test case considered, the agreement between results predicted with the MOM and detailed calculations with the KE appears to be reasonable, both for the flow field variables as well as the droplet size distributions.

\subsection{Introduction}

In condensing flows, such as observed in steam turbines or supersonic gas conditioners, the rapid expansion of the vapor generates a dispersion of small droplets via the process of homogeneous nucleation. Previously, the evolution of the droplet size distribution (DSD) was described by the so-called master equation, which is essentially a mass-conservation law in phase space, i.e., the space spanned by the physical position vector $\mathbf{x}$ and the droplet size [52], [101], [127]. Since the latent heat release by the condensing droplets affects the dynamics of the compressible flow, there is a strong two-way coupling present between the gaseous and liquid phases. The consequence of this strong coupling is that the fluid dynamics equations (FDE) for the flow field need to be solved simultaneously with the master equation for the liquid dispersion. This approach has been adopted in a number of past investigations on condensing steam, e.g., [112], [147], [130], [131], and also in chapter 3. It is, however, 
not favored for large-scale engineering applications, as the computational resources to obtain high resolution in both physical space and droplet size space are far too expensive to be practically viable. The usual practice is to employ a reduced model, viz. the so-called method of moments (MOM), in which moments of the droplet size distribution are used instead, as this yields a much smaller set of equations that needs to be solved. Since its first application to condensing nozzle flow by Hill in [45], the MOM has frequently been applied in numerical simulations involving either two- or three-dimensional geometries [96], [91], [53], unsteady condensing flows [1], [67], [68],[43], and/or viscous turbulent flows [108], [5].

The moment data obtained with the MOM includes, amongst others, the total droplet number density and the scaled liquid mass fraction of the two-phase mixture. The latter is of specific importance, since it allows the thermodynamic state variables, such as temperature and pressure, to be calculated from the mixture density and internal energy via appropriate equations of state (see chapter 2 ). In the original MOM [45], the variation in the liquid mass fraction is given by a source term which is an integral containing the product of the size-dependent droplet growth rate and the droplet size distribution. Since the latter is not available in the MOM, one is faced with the problem of extracting the average droplet growth rate from the moment data by some approximate means. This closure problem associated with the MOM dates back to the work of Stieltjes, who was one of the first to investigate this problem in a systematic fashion [105]. Besides condensing flow, the MOM has been widely applied in other branches of physics, notably within the field of aerosol dynamics [137], [16], [88], [102], [8], [9].

Over the years, various methods have been devised to approximately solve the closure problem, ranging from relatively simple approaches, to more complicated schemes. An example of a relatively simple approach is to evaluate all integrals involving the DSD based on an average droplet size, which is obtained from the ratio of two or more moments, as is done in Hill's MOM [45]. Clearly, this is not an unambiguous approach, as the average droplet size can be obtained using various combinations of moments. Moreover, such methods do not allow for control of the closure error, and as a result, success is limited to a number of special cases.

A more elaborate approach is to assume a generic shape for the size distribution (e.g., using log-normal distributions, or polynomial expansions), which contains parameters that can be calculated from the moment data [102], [68], [50], [49]. This technique is, however, not attractive for multi-modal or strongly skewed distributions, as a rather large set of moments is usually required to accurately reconstruct the droplet size distribution. Moreover, White [129] and McGraw et al. [76] have revealed that there are several classes of size distributions which share the same sets of moments, even though their shapes are entirely different. Reconstruction of the size distribution from a finite set of moments thus constitutes an ill-posed problem, if 
the range of possible DSD's is left unconstrained.

The latest advances in the MOM regard the so-called quadrature methods, in which numerical quadrature is used to approximate the integral representing the condensation source term. Several variants have been developed, such as the quadrature method of moments (QMOM, [75]), the direct quadrature method of moments (DQMOM, [70]), and the fixed interval quadrature method of moments (FIQMOM, [3], [36]). Quadrature methods are very attractive because they have shown to yield highly accurate approximations, and furthermore, the accuracy can formally be tuned as desired, with the penalty that for increased accuracy a larger set of moments needs to be employed.

The MOM efficiently generates a flow field which approximately encompasses the effects of condensation. By solving the master equation in a subsequent postprocessing step, the droplet size distribution is then determined along selected fluid trajectories. As demonstrated by Hagmeijer et al. in [42], such a strategy allows one to obtain details of the size distribution with very moderate computational effort. As shown by Sidin and Hagmeijer in [107], however, the closure error in the MOM can severely corrupt the solution for the size distribution, if simple closures, such as Hill's MOM, are used.

In this investigation Hill's MOM, the QMOM and DQMOM are evaluated for application in condensing flow models. The moment equations considered in this investigation are either derived from the general dynamic equation (GDE, [45]), or from the kinetic equation (KE) for the discrete droplet size distribution (Eq. (3.3) in chapter 3 ). Firstly, the closure approximations in the MOM are qualitatively evaluated, using key requirements identified from steady-state and transient characteristics of the KE. Attention is also focused on the robustness of the methods, as this is recognized to be a fundamental requirement for a successful application of the MOM. Finally, the influence of the closure error in the MOM is quantified by comparing the predicted flow field solution and reconstructed droplet size distributions with the benchmark solutions obtained by simultaneously solving the FDE and the KE.

\subsection{Moments and moment equations}

\subsubsection{Moment equations based on the kinetic equation}

The kinetic equation (KE) for the discrete droplet size distribution $\hat{\rho}_{n}(\mathbf{x}, t)$ is given by:

$$
\mathcal{L}\left(\hat{\rho}_{n}\right)=J_{n-1}-J_{n}, n=2,3, . .
$$

where $\mathcal{L}$ denotes the advection operator,

$$
\mathcal{L}(.)=\frac{\partial(.)}{\partial t}+\frac{\partial}{\partial x_{j}}\left(u_{j}(.)\right)
$$


and where $J_{n}=f_{n} \hat{\rho}_{n}-b_{n+1} \hat{\rho}_{n+1}$ is the condensation flux. The forward rate $f_{n}$ and backward rate $b_{n}$ depend on the droplet size, the vapor supersaturation $S$, and the vapor and droplet temperatures $T$ and $T_{n}$, respectively (see chapter 3). Although not strictly necessary for the derivation of the moment equations from the KE, here an isothermal condensation model will be assumed, i.e., $T_{n}=T$.

The $k^{t h}$-moment $v_{k}$ of the discrete droplet size distribution is now defined as:

$$
v_{k}(\mathbf{x}, t) \equiv \sum_{n=2}^{N} \hat{\rho}_{n}(\mathbf{x}, t) n^{k} .
$$

The zero-th moment $(k=0)$ represents the total number of droplets per unit volume of the two-phase mixture $\left(N_{l}\right)$, whereas the first moment $(k=1)$ corresponds with the mean density of the liquid phase $\left(\rho_{l}\right)$.

Application of the advection operator $\mathcal{L}$ to Eq. (4.3),

$$
\mathcal{L}\left(v_{k}\right)=\sum_{n=2}^{N} n^{k} \mathcal{L}\left(\hat{\rho}_{n}\right)=\sum_{n=2}^{N} n^{k}\left\{f_{n-1} \hat{\rho}_{n-1}-\left(f_{n}+b_{n}\right) \hat{\rho}_{n}+b_{n+1} \hat{\rho}_{n+1}\right\},
$$

followed by the manipulations:

$$
\sum_{n=2}^{N} n^{k} f_{n-1} \hat{\rho}_{n-1}=\sum_{n=2}^{N} f_{n} \hat{\rho}_{n}(n+1)^{k}+f_{1} \hat{\rho}_{1} 2^{k}-f_{N} \hat{\rho}_{N}(N+1)^{k},
$$

and:

$$
\sum_{n=2}^{N} n^{k} b_{n+1} \hat{\rho}_{n+1}=\sum_{n=2}^{N} b_{n} \hat{\rho}_{n}(n-1)^{k}-b_{2} \rho_{2}+b_{N+1} \hat{\rho}_{N+1} N^{k},
$$

with $\hat{\rho}_{N}, \hat{\rho}_{N+1} \rightarrow 0$ for $N \gg 1$, finally yields:

$$
\mathcal{L}\left(v_{k}\right)=\sum_{n=2}^{N} \Upsilon_{k}(n) \hat{\rho}_{n}+f_{1} \hat{\rho}_{1} 2^{k}-b_{2} \hat{\rho}_{2},
$$

with:

$$
\Upsilon_{k}(n)=\Upsilon_{k}(n, \mathbf{x}, t) \equiv f_{n}(\mathbf{x}, t)\left[(n+1)^{k}-n^{k}\right]+b_{n}(\mathbf{x}, t)\left[(n-1)^{k}-n^{k}\right] .
$$

As $f_{n}$ and $b_{n}$ are not polynomial functions (see chapter 3 ), the summation term involving $\Upsilon_{k}(n)$ and $\hat{\rho}_{n}$ in Eq. (4.5) can only be calculated exactly when the size distribution is known. Hence, the calculation of this sum from available moment sets constitutes a closure problem in the MOM. This, however, is not the only closure problem that needs to be solved, as the last term on the right-hand side of Eq. (4.5) contains the dimer number density $\left(\hat{\rho}_{2}\right)$, which cannot be readily extracted from the moment 
data. The second term on the right-hand side of Eq. (4.5) also contains the monomer concentration $\hat{\rho}_{1}$, but this is determined from the (partial) vapor mass density $\rho_{v}$ as: $\hat{\rho}_{1}=\rho_{v} / m_{1}$, with $m_{1}$ denoting the mass of a single monomer. Alternatively, Eq. (4.5) can be rewritten by using the flux $J_{1}=f_{1} \hat{\rho}_{1}-b_{2} \hat{\rho}_{2}$ to replace the dimer number density $\hat{\rho}_{2}$. This yields the following expression:

$$
\mathcal{L}\left(v_{k}\right)=\sum_{n=2}^{N} \Upsilon_{k}(n) \hat{\rho}_{n}+f_{1} \hat{\rho}_{1}\left(2^{k}-1\right)+J_{1},
$$

which is fully equivalent to Eq. (4.5).

If the lower boundary is taken at $n=n_{0}+1$ instead of $n=2$, i.e., if all droplets with $n \leq n_{0}$ are neglected, then the moments $\bar{v}_{k}$ are obtained from:

$$
\bar{v}_{k}(\mathbf{x}, t) \equiv \sum_{n=n_{0}+1}^{N} \hat{\rho}_{n}(\mathbf{x}, t) n^{k} .
$$

Application of the advection operator $\mathcal{L}$ yields the corresponding moment equation:

$$
\mathcal{L}\left(\bar{v}_{k}\right)=\sum_{n=n_{0}+1}^{N} \Upsilon_{k}(n) \hat{\rho}_{n}+f_{n_{0}} \hat{\rho}_{n_{0}}\left(n_{0}+1\right)^{k}-b_{n_{0}+1} \hat{\rho}_{n_{0}+1} n_{0}^{k}
$$

In case the size distribution is dominated by large droplets, then $n_{0} \gg 1$, allowing the function $\Upsilon_{k}(n)$ to be approximated by applying a first-order Taylor series expansion to Eq. (4.6), so that: $(n+1)^{k}-n^{k} \approx k n^{k-1}$, and: $(n-1)^{k}-n^{k} \approx-k n^{k-1}$. The first term on the right-hand side of Eq. (4.9) is then approximated as:

$$
\sum_{n=n_{0}+1}^{N} \Upsilon_{k}(n) \hat{\rho}_{n} \approx k \sum_{n=n_{0}+1}^{N} \dot{n} n^{k-1} \hat{\rho}_{n},
$$

where $\dot{n}(n)=f_{n}-b_{n}$ is the droplet growth rate in $n$-space, previously introduced in chapter 3. By applying the additional approximation:

$$
f_{n_{0}} \hat{\rho}_{n_{0}}\left(n_{0}+1\right)^{k}-b_{n_{0}+1} \hat{\rho}_{n_{0}+1} n_{0}^{k} \approx\left(f_{n_{0}} \hat{\rho}_{n_{0}}-b_{n_{0}+1} \hat{\rho}_{n_{0}+1}\right) n_{0}^{k}=J_{n_{0}} n_{0}^{k}
$$

to the last terms on the right-hand side of Eq. (4.9), the following approximation to Eq. (4.9) is obtained:

$$
\mathcal{L}\left(\bar{v}_{k}\right) \approx k \sum_{n=n_{0}+1}^{N} \dot{n} n^{k-1} \hat{\rho}_{n}+J_{n_{0}}\left(n_{0}\right)^{k}, n_{0} \gg 1,
$$

where the condensation flux $J_{n_{0}}$ at the lower boundary is an unknown for which closure is needed. 


\subsubsection{Moment equations based on the general dynamic equation}

If the dispersion predominantly consists of large droplets, then the KE can be approximated by the GDE, as previously shown in chapter 3. By incorporating the condensation flux at the lower boundary $n_{0}=\varepsilon n^{*}, \varepsilon>1$, into the GDE, the latter can be written as:

$$
\mathcal{L}(\hat{\rho})=-\frac{\partial}{\partial n}(\dot{n} \hat{\rho})+\hat{J}\left(n_{0}\right), \quad n \in\left[n_{0}^{-}, \infty\right\rangle,
$$

where $\hat{\rho}=\hat{\rho}(n, \mathbf{x}, t)$ is the continuous size distribution, $\hat{J}\left(n_{0}\right)$ is the condensation flux at the lower boundary, and $n_{0}^{-}<n_{0}$, with $n_{0}-n_{0}^{-} \ll 1$. The relevant boundary conditions are given by:

$$
\hat{\rho}\left(n_{0}^{-}\right)=0, \lim _{n \rightarrow \infty} \hat{\rho}(n)=0 .
$$

Assuming quasisteady-state condensation for supersaturated vapor, and instantaneous evaporation of droplets with $n<n_{0}$ (so-called denucleation), the condensation flux is given by:

$$
\hat{J}\left(n_{0}\right)=\delta\left(n-n_{0}\right)\left[J H\left(\dot{n}-\frac{D n_{0}}{D t}\right)+\hat{\rho}\left(\dot{n}-\frac{D n_{0}}{D t}\right) H\left(-\dot{n}+\frac{D n_{0}}{D t}\right)\right],
$$

with $\delta$ denoting the Dirac delta-function, $H$ the Heaviside step function,

$$
H(\xi)=1 \text { if } \xi>0 \text {, else } H(\xi)=0,
$$

and $J$ the steady state nucleation rate (see appendix B). Defining the moment $\hat{v}_{k}$ by:

$$
\hat{v}(\mathbf{x}, t)_{k} \equiv \int_{n_{0}^{-}}^{\infty} \hat{\rho}(n, \mathbf{x}, t) n^{k} d n,
$$

the corresponding moment equation becomes:

$$
\mathcal{L}\left(\hat{v}_{k}\right)=\hat{S}_{G, k}+\hat{S}_{N, k}+\hat{S}_{D, k},
$$

where:

$$
\begin{aligned}
& \hat{S}_{G, k}=\int_{n_{0}^{-}}^{\infty} \hat{\rho}(n) \dot{n}(n) n^{k} d n=k \int_{n_{0}^{-}}^{\infty} \hat{\rho}(n) \dot{n}(n) n^{k-1} d n, \\
& \hat{S}_{N, k}=J\left(n_{0}\right)^{k} H\left(\dot{n}\left(n_{0}\right)-\frac{D n_{0}}{D t}\right), \\
& \hat{S}_{D, k}=\hat{\rho}\left(n_{0}\right) n_{0}^{k}\left(\dot{n}\left(n_{0}\right)-\frac{D n_{0}}{D t}\right) H\left(-\dot{n}\left(n_{0}\right)+\frac{D n_{0}}{D t}\right) .
\end{aligned}
$$

The source terms on the right-hand side of Eq. (4.16) are related to droplet growth $\left(\hat{S}_{G, k}\right)$, nucleation $\left(\hat{S}_{N, k}\right)$, and denucleation $\left(\hat{S}_{D, k}\right)$, respectively. 
It is noted that for dispersions containing sufficiently large droplets, the value assigned to $n_{0}$ can be much larger than unity, so that the integral on the right-hand side of Eq. (4.17) differs only slightly from the discrete sum in Eq. (4.11). If additionally, the special case of quasisteady nucleation is considered, then:

$$
J_{n_{0}}=\hat{J}\left(n_{0}\right)=J
$$

and:

$$
\dot{n}\left(n_{0}\right)>0, D n_{0} / D t=0,
$$

which means that under such conditions, the difference between Eq. (4.11) and (4.16) vanishes.

In Hill's MOM, the moment equations are formulated in terms of the moments $\mu_{k}$ of the droplet radius distribution function $f(r, \mathbf{x}, t)$,

$$
\mu_{k}(\mathbf{x}, t)=\int_{r_{0}^{-}}^{\infty} f(r, \mathbf{x}, t) r^{k} d r
$$

with $r_{0}^{-}$denoting the droplet radius corresponding with $n_{0}^{-}$. Application of the moment transformation Eq. (4.20) to the GDE for the droplet radius distribution function (see chapter 3 ) yields the following moment equation:

$$
\mathcal{L}\left(\mu_{k}\right)=S_{G, k}+S_{L, k}+S_{N, k}+S_{D, k}
$$

where:

$$
\begin{aligned}
& S_{G, k}=k \int_{r_{0}^{-}}^{\infty} f(r) r^{k-1} \dot{r}_{m} d r, \\
& S_{N, k}=r_{0}^{k} J H\left(\dot{r}\left(r_{0}\right)-\frac{D r_{0}}{D t}\right), \\
& S_{D, k}=r_{0}^{k} f\left(r_{0}\right)\left(\dot{r}\left(r_{0}\right)-\frac{D r_{0}}{D t}\right) H\left(-\dot{r}\left(r_{0}\right)+\frac{D r_{0}}{D t}\right), \\
& S_{L, k}=-\frac{k}{3 \rho_{b}} \frac{D \rho_{b}}{D t} \mu_{k} .
\end{aligned}
$$

Similar to the source terms given in Eqs. (4.17) - (4.19), the source terms on the right-hand side of Eq. (4.21) are related to droplet growth $\left(S_{G, k}\right)$, nucleation $\left(S_{N, k}\right)$, and denucleation $\left(S_{D, k}\right)$. The additional term $S_{L, k}$ on the right-hand side of Eq. (4.21) accounts for the change in droplet radius due to liquid expansion. The droplet growth rate $\dot{r}$ contains contributions from mass accumulation $\left(\dot{r}_{m}\right)$ and liquid expansion $\left(\dot{r}_{e}\right)$, and is given by the expression:

$$
\dot{r}=\dot{r}_{m}+\dot{r}_{e}
$$


where:

$$
\dot{r}_{m}=\frac{r}{3 n} \dot{n} \quad, \quad \dot{r}_{e}=-\frac{r}{3 \rho_{b}} \frac{D \rho_{b}}{D t},
$$

and $\rho_{b}$ denotes the temperature dependent bulk liquid density (a material property). It is noted that Eq. (4.21) can also be derived from Eq. (4.16), by applying the transformation $n \rightarrow r$, where:

$$
r=r\left(n, \rho_{b}\right)=\left(\frac{n m_{1}}{\frac{4}{3} \pi \rho_{b}}\right)^{1 / 3} .
$$

Similar to the moment equation derived from the KE, there are two terms which require closure in Eq. (4.21), viz. $S_{G, k}$ and $S_{D, k}$. In many investigations employing the MOM, the closure problem has exclusively been associated with the integral in Eq. (4.22), whereas the denucleation term $S_{D, k}$ has been neglected [45], [1], [91], [5]. In the present investigation the same approach will be adopted, i.e., $S_{D, k}=0$.

The crucial information extracted from the MOM is the liquid mass fraction $g$, which is used in the equations of state for the two-phase mixture (see chapter 2 ). In Hill's MOM the first four moments $(k=0,1,2,3)$ of the radius distribution function are used to form a closed system of equations, together with the fluid dynamics equations (FDE) for the two-phase mixture. The third moment is of special significance, since it relates to the liquid mass fraction via the expression:

$$
g=\frac{4 \pi}{3} \frac{\rho_{b}}{\rho} \mu_{3}
$$

where $\rho$ is the mixture density. When the moments of the discrete droplet size distribution are used instead, a closed system is already obtained by employing the first two moments $(k=0,1)$, as now $g$ follows from the first moment,

$$
g=m_{1} v_{1} / \rho
$$

\subsection{Closure methods for the moment equations}

\subsubsection{Fourier reconstruction}

In principle, it is possible to reconstruct the droplet size distribution from moment data when a complete set of moments (i.e., for $k=0$ to $\infty$ ) is available. Formally, reconstruction proceeds via the characteristic function $\Psi(\omega)$, which is the Fourier transform of the size distribution [83]:

$$
\Psi(\omega)=\mathfrak{F}\{f(r)\}_{\omega}=\int_{-\infty}^{\infty} f(r) \exp (i \omega r) d r,
$$


with $f(r) \equiv 0$ for $r<r_{0}$. Replacing the integrand by its Taylor series expansion around $r=0$, i.e.,

$$
\exp (i \omega r)=\sum_{k=0}^{\infty} \frac{(i \omega)^{k}}{k !} r^{k}
$$

the characteristic function becomes:

$$
\Psi(\omega)=\sum_{k=0}^{\infty} \frac{(i \omega)^{k}}{k !} \mu_{k}
$$

Application of the inverse Fourier transform then yields:

$$
f(r)=\mathfrak{F}^{-1}\{\Psi(\omega)\}_{r}=\frac{1}{2 \pi} \int_{-\infty}^{\infty} \Psi(\omega) \exp (-i \omega r) d \omega=\frac{1}{2 \pi} \sum_{k=0}^{\infty} \frac{\mu_{k}}{k !} \int_{-\infty}^{\infty}(i \omega)^{k} \exp (-i \omega r) d \omega .
$$

By using a finite set of moments in Eq. (4.33), it is possible to determine the size distribution approximately. However, the problem is that a rather large number of moments $(k>100)$ is required to obtain reasonable accuracy, especially when the size distribution is characterized by strong gradients. As the required computational effort is of the same order as a direct solution of the master equation, one usually employs computationally less demanding closure methods in the MOM. Two variants of such methods are evaluated in this chapter.

\subsubsection{Closure Requirements}

Ideally, the MOM should provide the same moments as one would obtain from the KE. The moment associated with the liquid mass fraction is of particular importance, as it controls the coupling between the condensation process and the flow dynamics.

To facilitate this discussion, the Lagrangian form of the KE is employed,

$$
\frac{D \check{\rho}_{n}}{D t}=\check{J}_{n-1}-\check{J}_{n}, \quad n=2,3, . ., N-1
$$

with $\check{\rho}_{n}=\hat{\rho}_{n} / \rho$, and $\check{J}_{n}=J_{n} / \rho$. The boundary conditions for Eq. (4.34) are taken as $\check{\rho}_{1}=m_{1} \rho_{v} / \rho$ and $\check{\rho}_{N}=0$.

Eq. (4.34) can be compactly written as:

$$
\frac{D \check{\boldsymbol{\rho}}}{D t}=\mathbf{A}\left(\check{\boldsymbol{\rho}}-\check{\boldsymbol{\rho}}^{s t}\right),
$$


where the matrix $\mathbf{A}$ is given by:

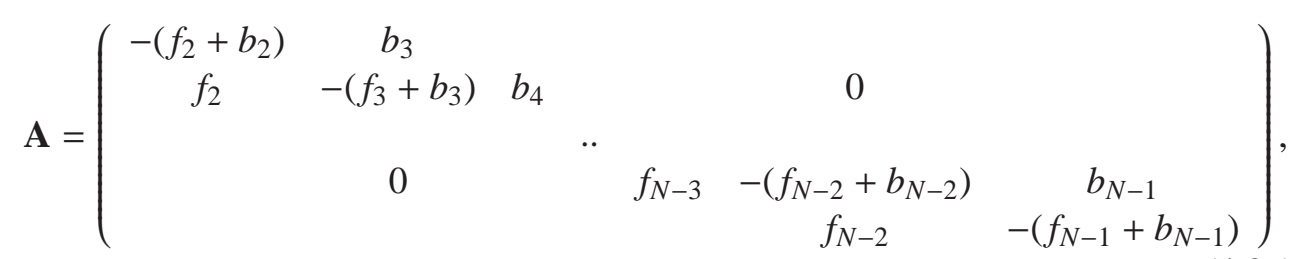

$\check{\boldsymbol{\rho}}=\left(\check{\rho}_{2}, \check{\rho}_{3}, . ., \check{\rho}_{N-1}\right)^{T}$, and $\check{\boldsymbol{\rho}}^{s t}=\mathbf{A}^{-1} \mathbf{b}$ is the steady state solution of the KE, with $\mathbf{b}=\left(f_{1} \check{\rho}_{1}, 0, \ldots, 0, b_{N} \check{\rho}_{N}\right)^{T}$.

For subsaturated vapor $(S<1), \check{\rho}_{N}^{e q}$ is practically zero if $N$ is chosen to be sufficiently large, meaning that the steady state KE-solution coincides with the equilibrium distribution, i.e., $\check{\boldsymbol{\rho}}^{\text {st }} \rightarrow \breve{\boldsymbol{\rho}}^{e q}$.

For supersaturated vapor $\check{\rho}_{N}^{e q}>0$, and thus $\check{\boldsymbol{\rho}}^{s t} \neq \check{\boldsymbol{\rho}}^{e q}$. As shown in chapter 3 , the KE can effectively be replaced by the GDE for large droplets, which means that:

$$
\frac{\partial}{\partial n}(\hat{\rho} \dot{n})=0 \Longrightarrow \hat{\rho} \sim \frac{1}{\dot{n}}
$$

for $n \gg 1$. As can be verified from Eqs. (3.4), (3.6), and (3.19), $\dot{n} \sim n^{2 / 3}$ for $n \gg 1$, meaning that for such droplet sizes $\hat{\rho}_{n} \sim n^{-2 / 3}$. If the size domain is not finite, however, i.e., $\Omega_{n}=[2, \infty\rangle$, then none of the moments $v_{k}$ are finite, since in the large droplet limit

$$
\sum_{n \in \Omega_{n}} \hat{\rho}_{n} n^{k} \approx \int_{\Omega_{n}} \hat{\rho}(n) n^{k} d n \sim \int_{\Omega_{n}} n^{-2 / 3} n^{k} d n=\left[\frac{n^{k+\frac{1}{3}}}{k+\frac{1}{3}}\right]_{2}^{\infty} \rightarrow \infty .
$$

This is clearly an unphysical situation which originates from the fact that for $S>1$ the DSD can only be in an actual steady state on a finite sized domain. If $\Omega_{n}$ is not finite, then there is a steady flux of droplets moving towards arbitrarily large droplets, which means that there is actually a quasisteady state solution for the DSD.

In conclusion:

(i) the KE has a finite and non-zero steady state-solution $\check{\boldsymbol{\rho}}^{s t}$ for $S<1$, which coincides with the equilibrium distribution;

(ii) for $S>1$, there exists a finite, non-zero quasisteady state solution, which yields finite moments on a finite domain.

The transient solution of the KE can be characterized by means of the eigenvalues $\lambda_{m}^{A}(t)$ and right eigenvectors $\mathbf{r}_{m}^{A}(t)$ of matrix $\mathbf{A}$. As the dimension of the quasilinear system in Eq. (4.35) can be very large (e.g., $N>10^{7}$ for condensing nozzle flows), calculation of the eigenproperties of $\mathbf{A}$ is not possible with present computational 
capabilities, and one would only be able to compute the eigenvalues of the system matrix corresponding with the sectional approximation of the KE (see Eq. (3.56) in chapter 3). Despite this difficulty, the sign of the eigenvalues can still be determined by using a similarity transformation to construct the symmetric tri-diagonal matrix Q:

$$
\begin{aligned}
& \mathbf{Q}=\mathbf{D}^{-1} \mathbf{A D}=
\end{aligned}
$$

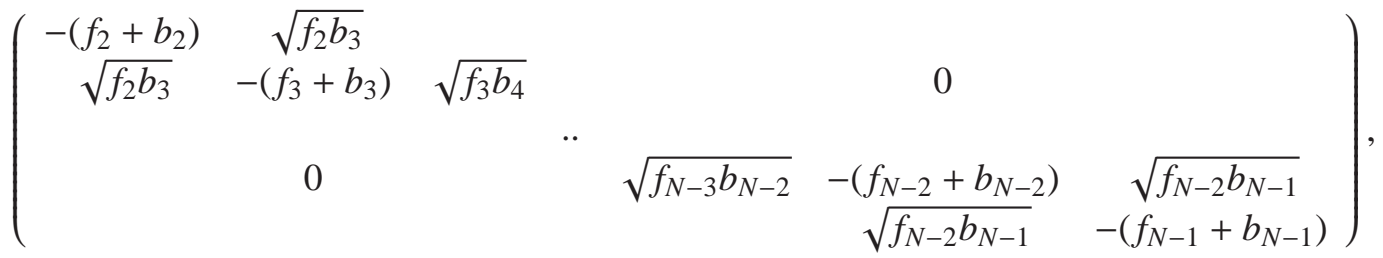

where $\mathbf{D}$ is a diagonal matrix with diagonal entries:

$$
d_{1}=1, d_{m}=d_{m-1} \sqrt{\frac{f_{m}}{b_{m+1}}} .
$$

The eigenvalues of $\mathbf{Q}$ and $\mathbf{A}$ are equal, but their eigenvectors are generally different. Calculation of the determinants of the principal sub-matrices of $-\mathbf{Q}$ reveals that these are all positive, e.g.,

$$
\begin{gathered}
\left|-\mathbf{Q}^{1}\right|=f_{2}+b_{2}>0 ;\left|-\mathbf{Q}^{2}\right|=\left|\begin{array}{cc}
f_{2}+b_{2} & -\sqrt{f_{2} b_{3}} \\
-\sqrt{f_{2} b_{3}} & f_{3}+b_{3}
\end{array}\right|=f_{2} f_{3}+f_{3} b_{2}+b_{2} b_{3}>0 ; \\
\left|-\mathbf{Q}^{m}\right|=\left(f_{m+1}+b_{m+1}\right)\left|-\mathbf{Q}^{m-1}\right|-f_{m} b_{m+1}\left|-\mathbf{Q}^{m-2}\right|>0, m \geq 3 .
\end{gathered}
$$

From this it follows that $\mathbf{- Q}$ is a positive definite matrix, which means that all eigenvalues are strictly positive [80]: $\lambda_{m}^{-Q}>0$, and consequently: $\lambda_{m}^{A}=\lambda_{m}^{Q}=-\lambda_{m}^{-Q}<0$. Thus it is established that KE drives the DSD towards the steady state solution that corresponds with $\check{\rho}^{\text {st }}$.

In view of this, it is desired that the reduced models satisfy the following requirements:

1. the reduced models should allow for a steady state solution for the moments;

2. the steady state solution should be stable, i.e., any departure from the steady state should result in a relaxation back towards it.

In addition, closure accuracy needs to be addressed. Let the closure error $\Delta_{k}$ be defined as the difference between the $k^{\text {th }}$-moment obtained from the KE and that obtained from the MOM. Then:

3. the reduced model should allow for control of the closure error, i.e., $\Delta_{k}=$ $\Delta_{k}(M)$, where $M$ is the size of the reduced model, viz. the number of moment equations; 
4. the reduced model should be consistent with the KE, i.e., the closure error should reduce to zero when the size of the reduced system is equal to that of the KE: $\Delta_{k}(M) \rightarrow 0$ for $M \rightarrow N$, with $N$ denoting the maximum droplet size.

In addition to the previous requirements, it is also desired that the reduced model be sufficiently robust for application in numerical codes. Although it is difficult to quantify this mathematically, the term robustness refers to the extent in which the reduced models can be solved, without the occurrence of physically impossible solutions, that would necessarily terminate a computation. Such a situation might occur during the solution of the moment equations when, for instance, a negative value for one of the moments is calculated. The usual remedy to this problem is to apply an adhoc correction method, which allows the computation to be continued. Robustness is a very important property, which is essential for the successful application of the reduced models in numerical codes.

Finally, it is also desired that the closure error is acceptably small for a reduced model of moderate size. This qualitative requirement can be assessed by comparing the MOM-solutions with those obtained with the KE. This can only be done for relatively simple geometries because accurate solutions of the KE are computationally very expensive.

\subsubsection{Hill's Method of Moments}

\section{Description of the method}

In Hill's MOM [45], the moment equations given by Eq. (4.21) are solved for $k=$ 0,1,2,3, with the integral in Eq. (4.22) approximated by:

$$
\int_{r_{0}^{-}}^{\infty} f(r) \dot{r}_{m} r^{k-1} d r \approx \dot{r}_{m}\left(\bar{r}_{H}\right) \int_{r_{0}^{-}}^{\infty} f(r) r^{k-1} d r=\dot{r}_{m}\left(\bar{r}_{H}\right) \mu_{k-1},
$$

where the Hill radius $r_{H}$ is defined by:

$$
r_{H} \equiv \sqrt{\frac{2 \mu_{2}}{\mu_{0}}}
$$

This closure relies on using the Hertz-Knudsen growth law, for which $\dot{r}_{m}$ tends to a size independent value for sufficiently large droplets. If the size distribution is dominated by such droplets, the approximation in Eq. (4.39) will obviously yield an accurate estimate of the droplet growth contribution $S_{G, k}$ in the MOM.

If the moments $v_{k}$ of the discrete droplet size distribution are used, a similar type of closure as in Hill's MOM can be devised. Based on the zero-th and first moments, 
the following moment equations need to be solved in conjunction with the FDE:

$$
\begin{aligned}
& \mathcal{L}\left(v_{0}\right)=J_{1} \\
& \mathcal{L}\left(v_{1}\right)=\sum_{n=2}^{N} \dot{n}(n) \hat{\rho}_{n}+f_{1} \hat{\rho}_{1}+J_{1}
\end{aligned}
$$

Using the average droplet size $\langle n\rangle \equiv v_{1} / v_{0}$, the sum over $n$ may be estimated by:

$$
\sum_{n=2}^{N} \dot{n}(n) \hat{\rho}_{n} \approx \dot{n}(\langle n\rangle) v_{0} .
$$

It is noted that the use of mean droplet sizes does not provide closure for the problem associated with the unknown value of $\hat{\rho}_{2}$ in Eq. (4.5), nor for the denucleation term in Eq. (4.24). In Hill's MOM denucleation is neglected entirely, and only nucleation at $r=r_{0}$ is taken into account by setting the local condensation flux equal to the steady state nucleation rate. For the moments of the discrete droplet size distribution, a similar approach is to replace the condensation flux $J_{1}$ with the steady state nucleation rate $J$. The corresponding approximation to the moment equation is then given by:

$$
\mathcal{L}\left(v_{k}\right)=\Upsilon_{k}(\langle n\rangle) v_{k-1}+f_{1} \hat{\rho}_{1}\left(2^{k}-1\right)+J .
$$

For the moments $\bar{v}_{k}$, defined by Eq. (4.8), a similar approximation is given by:

$$
\mathcal{L}\left(\bar{v}_{k}\right)=\Upsilon_{k}(\langle n\rangle) \bar{v}_{k-1}+J\left(n_{0}\right)^{k}
$$

The nucleation flux $J_{n_{0}}$ in Eq. (4.11) can similarly be approximated by the steady state nucleation rate.

\section{Properties of the method}

Assuming a frozen state of the vapor temperature and saturation, an evaluation of Hill's MOM for the moments $\mu_{k}$, with respect to the requirements that have been set forth previously, yields the following observations:

1. for $S<1: J=0$ and $\dot{r}\left(\bar{r}_{H}\right)<0$, so that in the steady state $\mu_{k}=0$ for $k>0$, whereas the zero ${ }^{\text {th }}$-moment $\mu_{0}$ remains equal to its value at the initial condition;

2. for $S>1: J>0$, and $\dot{r}\left(\bar{r}_{H}\right) \leq 0$ for $\bar{r}_{H} \leq r^{*}$, whereas $\dot{r}\left(\bar{r}_{H}\right)>0$ for $\bar{r}_{H}>$ $r^{*}$. Thus subcritical droplets will evaporate completely, whereas supercritical droplets continue growing, so that a steady state is not achieved;

3. the closure error cannot be made arbitrarily small, therefore, the method is inconsistent with the KE. 
The first two observations regarding the steady state solution for Hill's MOM are actually consistent with that of the GDE. As the latter does not allow for the existence of a non-zero steady state solution (see chapter 3 ), its corresponding moment equations should not be expected to do so.

Evaluation of the system of moment equations given by Eqs. (4.41) and (4.42), and augmented with Eq. (4.43), reveals the same deficiencies as observed above. Despite these shortcomings, Hill's MOM is frequently used in engineering applications, particularly because it is a very robust method. Usually, the only computational problem is associated with numerical overflow in the calculation of the Hill radius, when $\mu_{0}$ is very small. This is especially the case at the onset of condensation, where the number densities of droplets are very low. However, this problem can be easily remedied by delaying the calculation of the growth-term $S_{G, k}$ until the nucleation term $S_{N, k}$ yields sufficiently large values for $\mu_{0}$.

\subsubsection{The quadrature method of moments (QMOM)}

\section{Description of the method}

Although the QMOM has been documented in several articles, e.g., in [75] and [69], it has always been presented in mere algorithmic form, with relevant details of the underlying mathematics regarding the quadrature approximation being excluded. Unfortunately, this has led to a number of misconceptions on the QMOM, and furthermore, fundamental problems associated with the application of the QMOM have rarely been addressed [140]. Therefore, some of the relevant mathematical details on the QMOM will be discussed in this section.

The QMOM employs a numerical quadrature rule to estimate the moment source terms which contain integrals of the size distribution function over droplet size space. For a generic continuous distribution function $\omega(\xi)$, the approximation is:

$$
\int_{\Omega_{\xi}} \omega(\xi) \beta(\xi) d \xi=\sum_{m=1}^{M} w_{m} \beta\left(\xi_{m}\right)+\varepsilon_{M},
$$

where $w_{m}$ and $\xi_{m}$ denote the $m^{\text {th }}$ weight and abscissa, respectively, and $\varepsilon_{M}$ is the quadrature error. Here, the distribution function fulfills the role of a quadrature weight function (also known as a measure) over the domain $\Omega_{\xi}$ [89].

The quadrature rule also holds for a discrete sum,

$$
\sum_{\xi \in \Omega_{\xi}} \omega_{\xi} \beta(\xi)=\sum_{m=1}^{M} w_{m} \beta\left(\xi_{m}\right)+\varepsilon_{M},
$$

where $\omega_{\xi}$ represents a discrete measure. Eq. (4.47) follows from Eq. (4.46) because the discrete sum over $\Omega_{\xi}$ can be written as an integral of a function consisting of a sum of Dirac-delta functions. 
The $M$ weights and abscissas are determined from the requirement that the quadrature error be zero when $\beta(\xi)$ is replaced by any arbitrary polynomial with maximal order of $2 M-1$ [115]. Based on this requirement it can be derived that the abscissas $\xi_{m}$ are the roots of the $M^{\text {th }}$ orthogonal polynomial $P_{M}(\xi)$, whereas the weights follow from the following system of equations:

$$
\sum_{m=1}^{M} w_{m} P_{k}\left(\xi_{m}\right)=\left\{\begin{array}{rll}
\theta_{0} & : & \text { if } k=0 \\
0 & : & \text { otherwise }
\end{array},\right.
$$

where $\theta_{k}$ is the $k^{\text {th }}$-moment of the distribution function, defined as

$$
\theta_{k} \equiv \int_{\Omega_{\xi}} \omega(\xi) \xi^{k} d \xi, \text { or } \theta_{k} \equiv \sum_{\xi \in \Omega_{\xi}} \omega_{\xi} \xi^{k}
$$

for a continuous measure $\omega(\xi)$, and a discrete measure $\omega_{\xi}$, respectively. In principle, the weights and abscissas can be found by solving the following non-linear system of equations:

$$
\sum_{m=1}^{M} w_{m} \xi_{m}^{k}=\theta_{k}, \quad k=0,1, . ., 2 M-1,
$$

were it not that this is numerically a rather cumbersome task for large $M$. The weights and abscissas are therefore calculated by means of an alternative method which is numerically more convenient.

To describe this method, it is convenient to introduce the inner product of two functions $\eta(\xi)$ and $\zeta(\xi)$ with respect to the measure $\omega(\xi)$, or $\omega_{\xi}$, on the domain $\Omega_{\xi}$, with:

$$
\langle\eta(\xi), \zeta(\xi)\rangle \equiv \int_{\Omega_{\xi}} \eta(\xi) \zeta(\xi) \omega(\xi) d \xi,
$$

for a continuous measure $\omega(\xi)$, and

$$
\langle\eta(\xi), \zeta(\xi)\rangle \equiv \sum_{\xi \in \Omega_{\xi}} \eta(\xi) \zeta(\xi) \omega_{\xi}
$$

for a discrete measure $\omega_{\xi}$. The coefficients of the $k^{\text {th }}$ orthogonal polynomial $P_{k}(\xi)$ are obtained from the orthogonality requirement:

$$
\left\langle P_{m}(\xi), P_{k}(\xi)\right\rangle=0 \text { for } m=0,1, . ., k-1 .
$$

Using this property, the orthogonal polynomials can be recursively expressed as:

$$
P_{k+1}(\xi)=\left(\xi-a_{k}\right) P_{k}(\xi)-c_{k} P_{k-1}(\xi)
$$


with $P_{-1}(\xi) \equiv 0, P_{0}(\xi) \equiv 1$, and where the coefficients $a_{k}$ and $c_{k}$ are given by [89]:

$$
\begin{gathered}
a_{k}=\frac{\left\langle\xi P_{k}(\xi), P_{k}(\xi)\right\rangle}{\left\langle P_{k}(\xi), P_{k}(\xi)\right\rangle}, k=0,1, . . \\
c_{k}=\frac{\left\langle P_{k}(\xi), P_{k}(\xi)\right\rangle}{\left\langle P_{k-1}(\xi), P_{k-1}(\xi)\right\rangle}, k=1,2, . .
\end{gathered}
$$

Since $\omega(\xi), \omega_{\xi} \geq 0$ on $\Omega_{\xi}$, and not identically zero, it follows from these expressions and the inner-product definition in Eq. (4.51), and (4.52), that both $a_{k}$ and $c_{k}$ are strictly positive real numbers. As the abscissas $\xi_{k}$ are the roots of the $M^{\text {th }}$ orthogonal polynomial, it can also be shown that $\xi_{k} \in \Omega_{\xi}$, for all roots $k$ [115].

From the preceding expressions and the definition of the moments (Eq. (4.49)) it follows that it suffices to know the moments of the size distribution in order to calculate the coefficients $a_{k}$ and $c_{k}$. In actual implementation, these coefficients are conveniently calculated from the moment data by means of the product difference algorithm (PD), which was originally devised by Gordon [38].

Using the recursive expression in Eq. (4.54), the set of orthogonal polynomials $\mathbf{P}(\xi)=\left(P_{0}(\xi), P_{1}(\xi), . ., P_{M-1}(\xi)\right)^{T}$ can be compactly represented as:

$$
\xi \mathbf{P}(\xi)=\mathbf{T P}(\xi)+P_{M}(\xi) \mathbf{e}_{M-1},
$$

where the $M \times M$-matrix $\mathbf{T}$ is given by:

$$
\mathbf{T}=\left(\begin{array}{ccccccc}
a_{0} & 1 & & & & & \\
c_{1} & a_{1} & 1 & & & 0 & \\
& & & . . & & & \\
& 0 & & & c_{M-2} & a_{M-2} & 1 \\
& & & & & c_{M-1} & a_{M-1}
\end{array}\right),
$$

and where $\mathbf{e}_{M-1}$ is the $M$-dimensional unit vector: $\mathbf{e}_{M-1} \equiv(0,0, . ., 0,1)^{T}$. If $\xi$ is now replaced by $\xi_{k}$, which is both the $k^{\text {th }}$ abscissa as well as the $k^{\text {th }}$ root of $P_{M}(\xi)$, then Eq. (4.57) reduces to:

$$
\xi_{k} \mathbf{P}\left(\xi_{k}\right)=\mathbf{T P}\left(\xi_{k}\right)
$$

which resembles an eigenvalue problem with $\xi_{k}$ fulfilling the role of eigenvalue of matrix $\mathbf{T}$. To actually compute the eigenvalues, it is numerically more convenient to replace $\mathbf{T}$ with a symmetric tri-diagonal matrix $\mathbf{T}_{s}$ [89], since there are several established methods available to compute the eigenvalues of a tri-diagonal matrix in a robust manner [89]. The matrix $\mathbf{T}_{s}$ is obtained by the similarity transformation:

$$
\mathbf{T}_{s}=\mathbf{D T D}^{-1},
$$

where $\mathbf{D}$ is a diagonal matrix, with diagonal entries

$$
d_{1}=1, d_{m}=d_{m-1} \sqrt{c_{m-1}} .
$$


As a result of Eq. (4.60), matrix $\mathbf{T}_{s}$ is obtained as:

$$
\mathbf{T}_{s}=\left(\begin{array}{ccccccc}
a_{0} & \sqrt{c_{1}} & & & & & \\
\sqrt{c_{1}} & a_{1} & \sqrt{c_{2}} & & & 0 & \\
& & & . . & & & \\
& 0 & & & \sqrt{c_{M-2}} & a_{M-2} & \sqrt{c_{M-1}} \\
& & & & & \sqrt{c_{M-1}} & a_{M-1}
\end{array}\right) .
$$

Once the abscissas are known, the weights can be calculated by solving the linear system in Eq. (4.48). Alternatively, they can be obtained from the eigenvectors of $\mathbf{T}_{s}$. If $\mathbf{r}_{k}^{T_{s}}$ denotes the $k^{\text {th }}$ eigenvector of $\mathbf{T}_{s}$, which is scaled such that:

$$
\left\|\mathbf{r}_{k}^{T_{s}}\right\|=\theta_{0}
$$

then:

$$
w_{k}=\left(r_{k, 1}^{T_{s}}\right)^{2}
$$

where $r_{k, 1}^{T_{s}}$ is the first component of $\mathbf{r}_{k}^{T_{s}}$. A proof of this can be found in [115].

Implementation of the QMOM for the specific case of the moments $\mu_{k}$ of the radius distribution function (see Eq. (4.21)) is illustrated in Figure 4.1. For the moments $v_{k}$ corresponding with the KE, the implementation proceeds in similar fashion.

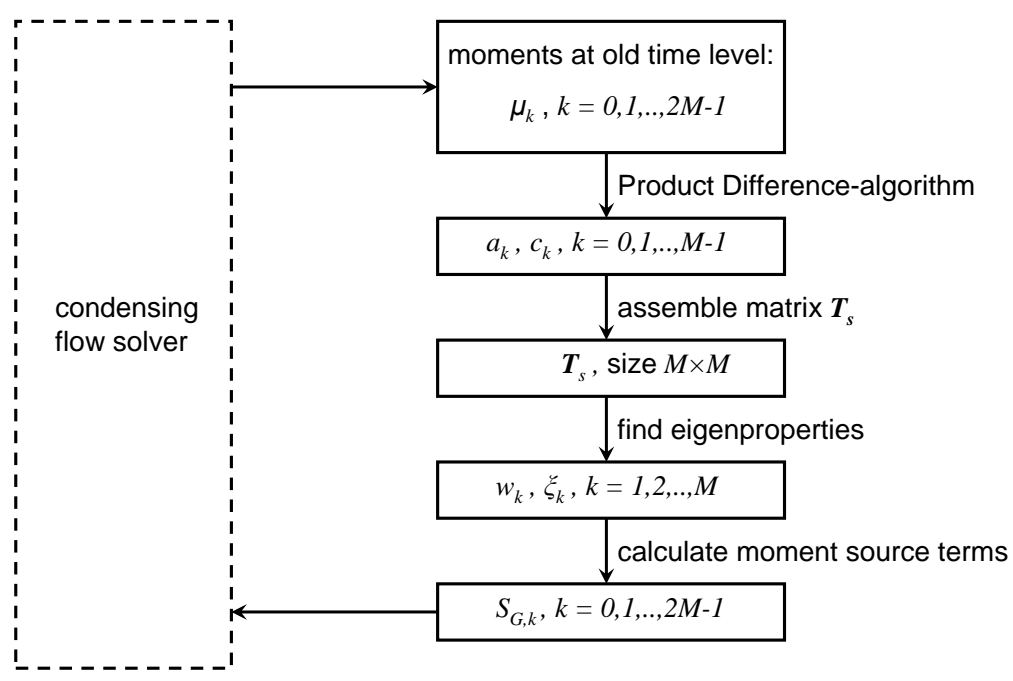

FIGURE 4.1: QMOM-implementation in a condensing flow solver. 


\section{Properties of the method}

\section{Realizability of the moment data}

It is well known that inversion of the moment data to compute the weights and abscissas is an ill-conditioned problem, and usually its application is not recommended in the literature [89]. The main problem concerns the calculation of the coefficients $a_{k}$ and $c_{k}$ from the moment data. For $c_{k}$ specifically, it appears that small perturbations in the moment set can result in negative values for this coefficient, which conflicts with Eq. (4.56), from which $c_{k}>0$. Negative $c_{k}$ results in non-physical solutions for the abscissas $\xi_{k}$, i.e., $\xi_{k} \notin \Omega_{\xi}$. This problem is directly linked to the so-called realizability of the moment set, which dates back to the work by Stieltjes [105]. The original moment problem conceived by Stieltjes consists of a number of queries with which a given moment set can be probed to determine specific information about the underlying distribution function. The query concerning the realizability of the moment set is:

For a given finite sequence of moments $\theta_{k}(k=0,1, . ., 2 M-1)$, does there exist a positive measure $\omega(\xi)$ on the domain $\Omega_{\xi}=\left[\xi_{0}, \infty\right\rangle$, of which the moments coincide with the given moment set?

The mathematical answer to this query is given by the following theorem, originally derived by Stieltjes*, and for which the proof is given in [105]:

\section{THEOREM:}

I. A necessary condition for the existence of a measure $\omega(\xi)$ on the domain $\Omega_{\xi}=$ $\left[\xi_{0}, \infty\right\rangle$, corresponding with the moment set $\theta_{k}(k=0,1, . ., 2 M-1)$ is that the determinants of the Hankel matrices $\mathbf{H}_{1}^{k}, \mathbf{H}_{2}^{k}$, defined by:

$$
H_{1, m n}^{k} \equiv \tilde{\theta}_{m+n}, H_{2, m n}^{k} \equiv \tilde{\theta}_{m+n+1}, \quad k=1,2, . ., M,
$$

and with:

$$
\tilde{\theta}_{k}=\tilde{\theta}_{k}\left(\theta_{0}, \theta_{1}, . ., \theta_{2 M-1}\right) \equiv \int_{\xi_{0}}^{\infty} \omega(\xi)\left(\xi-\xi_{0}\right)^{k} d \xi,
$$

be non-negative for all $k \in[1, M]$, i.e., $\left|\mathbf{H}_{1}^{k}\right| \geq 0$ and $\left|\mathbf{H}_{2}^{k}\right| \geq 0 \forall k \in[1, M]$.

II. In order for a measure $\omega(\xi)$ to exist whose spectrum is not reducible to a finite set of points, it is necessary and sufficient that $\left|\mathbf{H}_{1}^{k}\right|>0$ and $\left|\mathbf{H}_{2}^{k}\right|>0 \forall k \in$ $[1, M]$.

*Extensions of Stieltjes' moment problem to the domains $\mathbb{R}$ and $[0,1]$ are given by the Hamburger and Haussdorf moment problems, respectively [105] 
The modified moments $\tilde{\theta}_{k}$ can be calculated from the moment set $\theta_{k}$, and evidently, $\tilde{\theta}_{k}=\theta_{k}$ when $\xi_{0}=0$. Although a continuous measure has been used in the previous theorem, it can be readily demonstrated that its validity also holds for discrete measures, but with the modified moments now given by:

$$
\tilde{\theta}_{k}=\tilde{\theta}_{k}\left(\theta_{0}, \theta_{1}, . ., \theta_{2 M-1}\right)=\sum_{\xi=\xi_{0}+1} \omega_{\xi}\left(\xi-\xi_{0}\right)^{k}
$$

If the conditions set forth in the above theorem are not satisfied, then $\xi_{k} \notin \Omega_{\xi}$, which often means that one or more negative abscissas are calculated. This jeopardizes the calculation process, and is usually remedied in an adhoc fashion. One of the few investigations in which this, and other problems associated with the QMOM were addressed, was conducted by Wright in [140]. He discovered that the seemingly small errors introduced by so-called flux-limited interpolation methods which are used to calculate the state variables at cell-vertices in computational fluid dynamics (CFD) codes (see, e.g., [59] for an extensive exposition on interpolation schemes), result in non-realizable states of the moment sets. This can be illustrated by means of the following one-dimensional advection problem for the moments $\theta_{k}$ :

$$
\frac{\partial \theta_{k}}{\partial t}+u \frac{\partial \theta_{k}}{\partial x}=0
$$

with $u$ representing a constant advection velocity. Adopting a finite-volume spatial discretization and forward Euler time integration, the numerical solution for Eq. (4.68) is given by:

$$
\bar{\theta}_{k, i}^{n+1}=\bar{\theta}_{k, i}^{n}-\Gamma_{i}^{n}\left[\bar{\theta}_{k, i+1 / 2}^{n}-\bar{\theta}_{k, i-1 / 2}^{n}\right],
$$

where $\bar{\theta}_{k, i}^{n}$ is the numerical approximation of $\theta_{k}$ at position $x_{i}$ and time $t^{n}$, and where $\Gamma_{i}^{n}$ is the so-called CFL-number (from Courant, Friedrichs and Lewy, [23]), given by:

$$
\Gamma_{i}^{n}=\frac{u\left(t^{n+1}-t^{n}\right)}{x_{i+1 / 2}-x_{i-1 / 2}}
$$

Eq. (4.69) reveals that the solution for the moment set at the new time level $t^{n+1}$ is a linear combination of the moment sets $\bar{\theta}_{k, i}^{n}, \bar{\theta}_{k, i+1 / 2}^{n}$, and $\bar{\theta}_{k, i-1 / 2}^{n}$ at the old time level $t^{n}$. If each of these moment sets are realizable, and $\bar{\theta}_{k, i}^{n+1}>0$, then the moment set at the new time level will also be realizable. This can be deduced by noting that each realizable moment set $\bar{\theta}_{k, i}^{n}$ corresponds to an underlying distribution function, i.e., there exists a distribution $\omega_{i}^{n}(\xi)$ on a domain $\Omega_{\xi}$ for which

$$
\int_{\Omega_{\xi}} \omega_{i}^{n}(\xi) \xi^{k} d \xi=\bar{\theta}_{k, i}^{n}, \forall k .
$$


A new distribution $\omega_{i}^{n+1}(\xi)$ can be introduced, which is given by:

$$
\omega_{i}^{n+1}(\xi)=\omega_{i}^{n}(\xi)-\Gamma_{i}^{n}\left[\omega_{i+1 / 2}^{n}(\xi)-\omega_{i-1 / 2}^{n}(\xi)\right] .
$$

If $\omega_{i}^{n+1}(\xi)>0 \forall \xi \in \Omega_{\xi}$, then each $k^{t h}$ moment of $\omega_{i}^{n+1}(\xi)$ exactly coincides with $\bar{\theta}_{k, i}^{n+1}$, which means that the updated moments on the left-hand side of Eq. (4.69) indeed constitute a realizable set. If $\bar{\theta}_{k, i}^{n}>0$ for all times $t^{n}$, it is concluded that the updated moment sets will remain realizable during the entire simulation process.

If at least one of the moment sets on the right-hand side of Eq. (4.69) is not realizable, then the possibility exists that the updated solution $\bar{\theta}_{k, i}^{n+1}$ is also not realizable, thus causing complications in further computations with this moment set. In numerical codes, each moment $\bar{\theta}_{k, i \pm 1 / 2}^{n}$ associated with the cell-vertex $x_{i \pm 1 / 2}$ is usually obtained by interpolation from the solutions $\bar{\theta}_{k, i}^{n}$ stored at the neighboring cell-centers, i.e.,

$$
\bar{\theta}_{k, i \pm 1 / 2}^{n}=F\left(\bar{\theta}_{k, i}^{n}, \bar{\theta}_{k, i \pm 1}^{n}, . .\right)
$$

with $F$ resembling the interpolation operator. Since $F$ operates separately on each moment, the ratio $\psi_{k}=\bar{\theta}_{k, i+1 / 2}^{n} / \bar{\theta}_{k, i+j}^{n}$ between the vertex-solutions and the centersolutions does not need to be the same for all $k$, specifically if the interpolation method is non-linear. As a consequence, it is generally not possible to express the moment set at the vertex as a linear combination of the moment sets at neighboring cells, with uniform weighting factors $\psi_{k}$ for all $k$. Therefore, there is no guarantee that the interpolated vertex-moments constitute realizable sets, which initiates corruption of the moment data, with the eventual consequence of premature termination of the computation process.

To circumvent this particular problem, one can simply restrict the numerics to firstorder upwind interpolation, which guarantees the realizability of the vertex-moments, but has the penalty that the advection scheme becomes very diffusive. Wright has proposed a number of alternative solution strategies in [140], which includes a.o. the application of so-called augmented advection schemes that are based on uniform weighting of the moments at the cell-centers. Specifically, this means that a reference moment $\theta_{i, r e f}$ is first chosen for which the interpolation is carried out in the usual way. Next, the weighting factors $\psi_{i, j}$ are calculated, which represent the ratio between the updated reference moment $\bar{\theta}_{i, r e f}^{n+1}$ and the neighboring cell-center solutions $\bar{\theta}_{i+j, r e f}^{n}$ at the old time level, i.e.,

$$
\psi_{i, j}=\frac{\bar{\theta}_{i, r e f}^{n+1}}{\bar{\theta}_{i+j, r e f}^{n}} .
$$

The remaining moments at the new time level are then obtained by linear weighting of neighboring solution data, according to:

$$
\bar{\theta}_{i, k}^{n+1}=\sum_{j} \psi_{i, j} \bar{\theta}_{i+j, k}^{n}
$$


Given the typically strong variations in the moments such as observed in condensing flow [131], [107], the preceding augmented advection scheme will not handle the advection of the moments properly.

\section{Accuracy of the method}

Besides the realizability problem, it is also true that the weights and abscissas depend very sensitively on the moment data. This means that, even though the predicted moment sets may retain the realizability property, it still may be the case that, due to the ill-conditioned nature of the inversion operation, small errors in the moments may lead to unacceptably large errors in the weights and abscissas [89]. Furthermore, the loss of accuracy becomes more pronounced as the quadrature order $M$ is increased, and in practice, an almost complete loss of accuracy is experienced beyond $M=12$ [89]. This observation apparently conflicts with the outcome of various previous investigations in which QMOM was found to be a very accurate means for closure of the moment problem, e.g., in [36], [69]. However, it is noted that in those investigations, either advection was not included, simple integrand functions were used in the moment source terms, or that validation was carried out by using measurement data from experiments as the benchmark for comparison. Moreover, a rigorous mathematical analysis on the accuracy of the QMOM is still lacking.

The accuracy-properties of quadrature methods in numerical integration are well documented in the literature (see, e.g., [115]), but this usually regards cases for which the measure $\omega(\xi)$ is explicitly given. For such type of problems it can be demonstrated that the quadrature error $\varepsilon_{M}$ in Eq. (4.46) decreases with increasing $M$, if the Taylor series expansion of the integrand function $\beta(\xi)$ converges. If now the $\mathrm{QMOM}$ is considered in the situation when a moment source term of the form:

$$
S_{k}=\int_{\Omega_{\xi}} \beta(\xi) \xi^{k} \omega(\xi) d \xi, \quad k=0,1, . ., 2 M-1
$$

is to be calculated for a set of $2 M-1$ moment equations, then obviously, the quadrature error would be zero if the function $\beta(\xi) \xi^{k}$ represents a polynomial of maximum order $2 M-1$. If, hypothetically, $\beta(\xi)$ is given by a polynomial of order $Q$, then a zerovalued quadrature error for the $k^{\text {th }}$ moment source term requires that $k+Q \leq 2 M-1$. This can only be achieved for the moments with $k=0,1, . ., 2 M-1-Q$, whereas generally, the error is not zero for $k=2 M-Q, . ., 2 M-1$. The latter set of moments is thus corrupted by the quadrature error introduced in their corresponding source terms. Since the complete set of moments $(k=0,1, . ., 2 M-1)$ is used to compute the weights and abscissas, the low order moments will, eventually, also be affected by the quadrature error in the higher-order moments. Currently, the extent to which this affects the total accuracy of the QMOM can only be revealed by means of rigorous comparison with moment data from detailed solutions of the KE. 


\section{Consistency with the $\mathrm{KE}$}

As the QMOM does not provide closure for the dimer concentration $\hat{\rho}_{2}$ in Eq. (4.5), the QMOM is not consistent with the KE. To achieve consistency, the QMOM needs to be augmented by some other type of closure method that allows $\hat{\rho}_{2}$ to be estimated. Besides this inconsistency, the QMOM cannot be applied to the moments of a zero-distribution, whereas such distributions can be described without any difficulty by the master equations (KE and GDE). The computation of weights and abscissas cannot be performed for zero-distributions, because the coefficients $a_{k}$ and $c_{k}$ in Eqs. (4.55) and (4.56) cannot be determined. This is a serious deficiency in the QMOM, which has only been solved by adhoc methods, such as, e.g., by assigning random small values to the initial weights and abscissas, as suggested in [70]. It is noted that due to this problem, the onset of condensation cannot be handled properly when the QMOM corresponding with the GDE is used, as the distribution function is initially zero everywhere. In contrast, the QMOM based on the KE has the advantage that an equilibrium distribution can be specified at condensation-onset, which allows the proper initialization of the QMOM. For this reason, the QMOM will only be applied to the moment equations derived from the KE.

\section{Steady state and transient characteristics}

With respect to the moment equations corresponding with the KE (see Eq. (4.4)), the steady state and transient behavior can best be illustrated by considering the relatively simple case of $M=1$ (i.e., one abscissa), with the vapor temperature and mass density fixed at constant values. This yields the following set of moment equations:

$$
\begin{aligned}
& \frac{D \check{v}_{0}}{D t}=f_{1} \check{\rho}_{1}-b_{2} \check{\rho}_{2} \\
& \frac{D \check{v}_{1}}{D t}=2 f_{1} \check{\rho}_{1}-b_{2} \check{\rho}_{2}+w \dot{n}(\xi),
\end{aligned}
$$

where $D / D t$ is the material derivative, $\breve{v}_{k}=v_{k} / \rho$, and the weight and abscissa are given by $w=\breve{v}_{0}$, and $\xi=\check{v}_{1} / \check{v}_{0}$, respectively. Here, the indices of the weight and abscissa have been dropped for the sake of convenience.

It is observed that these moment equations are nonlinear, in contrast to the $\mathrm{KE}$ (see Eq. (4.35)). Eqs. (4.74) and (4.75) can be replaced with the equivalent expressions:

$$
\begin{aligned}
& \frac{D w}{D t}=f_{1} \check{\rho}_{1}-b_{2} \check{\rho}_{2} \\
& \frac{D \xi}{D t}=2 \frac{f_{1}}{w} \check{\rho}_{1}-\frac{f_{2}}{w}+\dot{n}(\xi) .
\end{aligned}
$$

When the vapor is subsaturated $(S<1)$, with the initial droplet size distribution equal to the equilibrium size distribution (see Eq. (3.7)), then the weight $w^{e q}$ and abscissa 
$\xi^{e q}$ can be calculated from the initial moments, using the QMOM-algorithm depicted in Figure 4.1. The equilibrium distribution remains invariant when it is marched in time using the KE. If instead, the weight and abscissa are marched in time using Eqs. (4.76) and (4.77) respectively, then by virtue of equilibrium:

$$
f_{1} \check{\rho}_{1}-b_{2} \check{\rho}_{2}^{e q}=0
$$

which leads to:

$$
\begin{aligned}
& \frac{D w}{D t}=0 \\
& \frac{D \xi}{D t}=\frac{f_{1}}{w} \check{\rho}_{1}+\dot{n}(\xi) .
\end{aligned}
$$

Thus $w(t)=w^{e q}$, but $\xi(t)$ changes in time, as the right-hand side of Eq. (4.79) is not necessarily zero.

The right-hand side $F(\xi)$ of Eq. (4.79),

$$
F(\xi) \equiv \frac{f_{1}}{w} \check{\rho}_{1}+\dot{n}(\xi),
$$

contains the growth rate $\dot{n}(n)$, evaluated at the abscissa $n=\xi$. For $S<1$, it follows from Eq. (3.19) that $\dot{n}(\xi)<0$, and that $\dot{n}(\xi)$ has a global maximum at, say, $\xi=\xi_{\max }$. Therefore, $F^{\prime}(\xi)>0$ for $\xi<\xi_{\text {max }}, F^{\prime}(\xi)<0$ for $\xi>\xi_{\max }$, and $F^{\prime \prime}(\xi)<0$ for all $\xi$. This means that at most two equilibrium points exist. Let $\xi_{e}$ be an equilibrium point, i.e., $F\left(\xi_{e}\right)=0$. If $\Delta \xi$ denotes the deviation from equilibrium, i.e.,

$$
\Delta \xi \equiv \xi-\xi_{e},
$$

then:

$$
\frac{D \Delta \xi}{D t}=F^{\prime}\left(\xi_{e}\right) \Delta \xi+\frac{1}{2} F^{\prime \prime}\left(\xi_{e}\right) \Delta \xi^{2}+O\left(\Delta \xi^{3}\right) .
$$

Hence, when two equilibrium points exist, one of them is unstable. Also, when one equilibrium point exist, it is unstable.

When $S>1$, then $\dot{n}^{\prime}(\xi)>0$, with $\dot{n}\left(n^{*}\right)=0$. Therefore $F^{\prime}(\xi)>0$ for all $\xi$, which means that there exists one equilibrium point which is unstable.

Thus in conclusion, the QMOM does not guarantee the existence of an equilibrium solution for the abscissa when $M=1$.

\section{Revised QMOM}

For $S<1$, a steady state solution for the moments is not guaranteed, even when the initial condition corresponds with the moment data for an equilibrium size distribution. In order to remove this deficiency, it is proposed to modify the moment equations in the following way. Firstly, it is recognized that for an equilibrium, or steady 
state distribution, the right-hand side of Eq. (4.1) is identically zero: $J_{n-1}^{0}-J_{n}^{0}=$ $0 \forall n \in \Omega_{n}$, where the superscript ' 0 ' refers to either equilibrium or steady state conditions. This means that the terms $J_{n-1}^{0}-J_{n}^{0}$ can be added to the right-hand side of Eq. (4.1):

$$
\mathcal{L}\left(\hat{\rho}_{n}\right)=J_{n-1}-J_{n}-\left(J_{n-1}^{0}-J_{n}^{0}\right),
$$

where $J_{n}^{0}=f_{n} \hat{\rho}_{n}^{0}-b_{n+1} \hat{\rho}_{n+1}^{0}$. The corresponding moment equation thus becomes:

$$
\mathcal{L}\left(v_{k}\right)=\sum_{n=2}^{N} \Upsilon_{k}(n) \hat{\rho}_{n}-\sum_{n=2}^{N} \Upsilon_{k}(n) \hat{\rho}_{n}^{0}-b_{2}\left(\hat{\rho}_{2}-\hat{\rho}_{2}^{0}\right),
$$

or equivalently,

$$
\mathcal{L}\left(v_{k}\right)=\sum_{n=2}^{N} \Upsilon_{k}(n) \hat{\rho}_{n}+J_{1}-\left(\sum_{n=2}^{N} \Upsilon_{k}(n) \hat{\rho}_{n}^{0}+J_{1}^{0}\right),
$$

where $J_{1}^{0}=0$ for equilibrium conditions, and $J_{1}^{0}=J$ for steady state conditions. Applying the quadrature approximation to Eq. (4.82) thus leads to:

$$
\mathcal{L}\left(v_{k}\right)=\sum_{m=1}^{M} w_{m} \Upsilon_{k}\left(\xi_{m}\right)+J_{1}-\left(\sum_{m=1}^{M} w_{m}^{0} \Upsilon_{k}\left(\xi_{m}^{0}\right)+J_{1}^{0}\right),
$$

which will be referred to as the revised QMOM. It is noted that the revised form of the moment equation given by Eq. (4.82) can also be used for other closure methods besides the QMOM.

It is noted that although the sum of the last terms within brackets on the right-hand side of Eq. (4.82) is zero, the corresponding term in Eq. (4.83) is not. It is easily verified from Eq. (4.83) though, that the moments and abscissas will remain invariant when the initial conditions correspond with an equilibrium distribution, which is in agreement with the transient behavior of the KE. However, it is noted that, in general, the transient solution for the weights and abscissas corresponding with the revised QMOM behaves similarly to those of the QMOM.

\subsubsection{The direct quadrature method of moments (DQMOM)}

The DQMOM utilizes the same quadrature rule as the QMOM to approximate the moment source terms. However, instead of solving the moment equations, a transformation is applied to convert these into balance equations for the weights $w_{k}$ and abscissas $\xi_{k}$. The latter set of equations is derived by substituting the quadrature expression in Eq. (4.50) for $v_{k}$ in Eq. (4.5), followed by expanding its left-hand side. To obtain a conservative transport equation for both the weights and abscissas it is 
convenient to replace the abscissas by the so-called weighted abscissas $\bar{\xi}_{k}$, defined by:

$$
\bar{\xi}_{k} \equiv w_{k} \xi_{k}
$$

Elaboration of the moment equation then yields:

$$
(1-k) \sum_{m=1}^{M} \xi_{m}^{k} \mathcal{L}\left(w_{m}\right)+k \sum_{m=1}^{M} \xi_{m}^{k-1} \mathcal{L}\left(\bar{\xi}_{m}\right)=S_{k},
$$

where $S_{k}$ is the right-hand side of either Eq. (4.5), or Eq. (4.82). By taking a set of $2 M$ moment equations, a unique solution for $\mathcal{L}\left(w_{m}\right)$ and $\mathcal{L}\left(\bar{\xi}_{m}\right)$ can be obtained. It is noted that to obtain a closed set of equations, it is not necessary to take the first $2 M$ moment equations, i.e., from $k=0$ to $k=2 M-1$. To maintain consistency with the QMOM, however, the moment set is restricted to the first $2 M$ moments. As a result, the system of balance equations for the weights and weighted abscissas is compactly given by:

$$
\mathbf{A}(\mathcal{L}(\mathbf{w}), \mathcal{L}(\overline{\boldsymbol{\xi}}))^{T}=\mathbf{S},
$$

where: $\mathbf{w}=\left(w_{1}, w_{2}, . ., w_{M}\right)^{T}, \bar{\xi}=\left(\bar{\xi}_{1}, \bar{\xi}_{2}, . ., \bar{\xi}_{M}\right)^{T}, \mathbf{S}=\left(S_{0}, S_{1}, . ., S_{2 M-1}\right)^{T}$, and the $2 M \times 2 M$-matrix $\mathbf{A}$ is given by:

$\mathbf{A}=\left(\begin{array}{cccccc}1 & . . & 1 & 0 & . . & 0 \\ 0 & . . & 0 & 1 & . . & 1 \\ -\xi_{1}^{2} & . . & -\xi_{M}^{2} & 2 \xi_{1} & . . & 2 \xi_{M} \\ : & . . & : & : & . . & : \\ 2(1-M) \xi_{1}^{2 M-1} & . . & 2(1-M) \xi_{M}^{2 M-1} & (2 M-1) \xi_{1}^{2(M-1)} & . . & (2 M-1) \xi_{M}^{2(M-1)}\end{array}\right)$

Having chosen $M$, the system of equations given by Eq. (4.86) can be solved for $\mathcal{L}(\mathbf{w}), \mathcal{L}(\bar{\xi})$, and subsequently, the weights and abscissas can be determined by solving these balance equations.

\section{Properties of the method}

The DQMOM inherits the same properties of the QMOM, as discussed previously. The only difference between these two methods is that realizability of the moment set is no longer a problem in the DQMOM, as the weights and abscissas are directly determined, without using the moments. All other problems associated with accuracy, steady state and transient behavior, as well as the failure to deal with zerodistributions, are identical to that of the QMOM. 


\subsection{Numerical evaluation of closure methods}

\subsubsection{Global procedure}

In contrast to what is claimed in much quoted references (see, e.g., [75], [70]), it was not possible to apply the QMOM nor the DQMOM successfully in the present investigation, due to the various problems that have been discussed in the preceding sections. For the two test cases that were presented in chapter 3 (as well as several others), the DQMOM consistently generated non-physical abscissas which prematurely terminated the simulations. As a consequence, an evaluation of the accuracy of the DQMOM for condensing flows cannot be given here, and thus, only Hill's MOM for $\mu_{k}$, or the MOM for $\bar{v}_{k}$ will be considered.

To quantify the accuracy of these moment methods, the condensing nozzle flow considered in chapter 3 (test case 2), will be employed. For the sake of clarity, the solutions predicted with the various methods are indexed as follows:

- index ' $K E$ ' refers to variables predicted by simultaneously solving the FDE with the KE;

- index 'HillMOM' refers to variables predicted by simultaneously solving the FDE with Eq. (4.21) for the moments $\mu_{0}, \mu_{1}, \mu_{2}$, and $\mu_{3}$, using the approximation given in Eq. (4.39), and by setting $S_{D, k}=0$ (i.e., denucleation is neglected);

- index 'MOMKE' refers to variables predicted by simultaneously solving the FDE with Eq. (4.45) for the moments $\bar{v}_{0}$ and $\bar{v}$. The lower boundary is set to either $n_{0}=3$ or $n_{0}=5$.

The relative difference $\Delta q^{\prime}$ between solution variable $q^{\prime}$ obtained with the FDE/MOM and $q^{K E}$ predicted with the $\mathrm{FDE} / \mathrm{KE}$, is defined as follows:

$$
\Delta q^{\prime} \equiv 100 \% \times \frac{q^{\prime}-q^{K E}}{q^{K E}},
$$

where the prime can be replaced by either 'HillMOM' or 'MOMKE'.

\subsubsection{Results and discussion}

The flow field variables predicted with the MOM are shown in Figures 4.2.a-d, along with the benchmark solution obtained by solving the KE and FDE simultaneously. For the KE, the sticking probability is set to unity $\left(\alpha_{n}=1\right)$, and the backward rates are calculated by using the Courtney equilibrium distribution (see chapter 3 ). The relative differences shown in Figures 4.3.a-d correspond with the solutions shown in Figures 4.2.a-d. Both sets of figures clearly show that the largest differences between 
the MOM-predictions and the benchmark solution occur within the nucleation zone, located between $x \approx 0.016 \mathrm{~m}$ and $x \approx 0.042 \mathrm{~m}$. The large differences in $g$ occur for $x<0.016 m$, i.e., upstream of the nucleation zone, but this region can be neglected because condensation effects are of minor importance in this region. Downstream of the nucleation zone $(x>0.042 m)$ the relative difference in each variable is consistently much smaller, with $\Delta T<0.2 \%$ and $\Delta g<0.5 \%$ at the nozzle exit. These observations are a consequence of the two-way coupling between the FDE and the $\mathrm{KE}$ on one hand, and the FDE and moment equations on the other hand. The two-way coupling occurs due to the effects of latent heat release and vapor depletion, and their influence can be described as follows. At the onset of condensation, the liquid mass fraction rises steeply, which simultaneously increases the temperature and lowers the vapor density. As a consequence, the saturation ratio $S$ decreases rapidly, which in turn slows down the rate of liquid production, as can be observed in Figures 4.2.c and $\mathrm{d}$. The approximations associated with the MOM will likely result in either too low or too high rates of liquid production, compared with the benchmark solution. Initially, this will lead to relatively large differences between both sets of solutions, but as the vapor saturation adjusts itself to the variation in the liquid mass fraction, the closure errors in the MOM will, to a certain extent, be compensated for, which explains why the liquid mass fraction profiles almost coincide near the nozzle exit.

The differences between the FDE/MOM- and FDE/KE-solutions are a consequence of the closure errors in the MOM, and the large-droplet approximation in Hill's MOM. Despite these approximations, the MOM gives reasonably accurate predictions, especially when noting that the differences between results obtained with various master equations (see, e.g., the temperature- and liquid mass fraction profiles in Figure 3.23) can be significantly larger. Given that the number of equations to be solved for the MOM is about a factor of 100 smaller than the number of equations to be solved for the KE, the MOM constitutes a very good trade-off between accuracy and computational expense.

Of course, the apparent drawback associated with the MOM is that the droplet size distribution remains unknown. However, the size distribution can be approximately reconstructed by solving the KE in a postprocessing step, using the flow field data generated by the MOM. This has been demonstrated in [42], where the GDE was used instead of the KE. Application of this reconstruction is demonstrated by computing the droplet number densities at various nozzle positions, as shown in Figures 4.4.a-d where the corresponding droplet radius distribution functions are plotted. Here, the index 'HillMOM' refers to the radius distribution which has been reconstructed by solving the KE, using the velocity, temperature and saturation profiles computed with Hill's MOM. Similarly, 'MOMKE' refers to the radius distribution reconstructed by means of the KE, using the flow field data predicted with the MOMKE. The agreement of the various size distributions is fairly good, considering that the saturation 


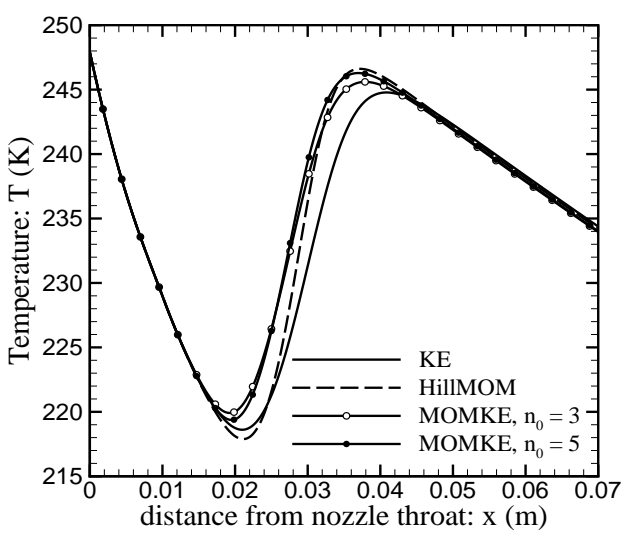

(a)

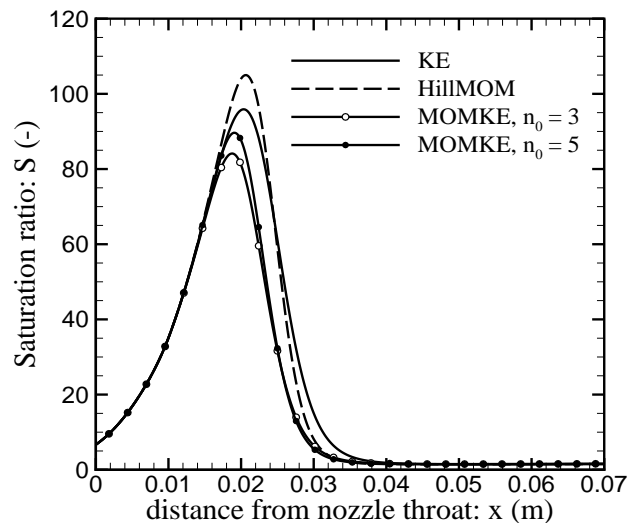

(c)

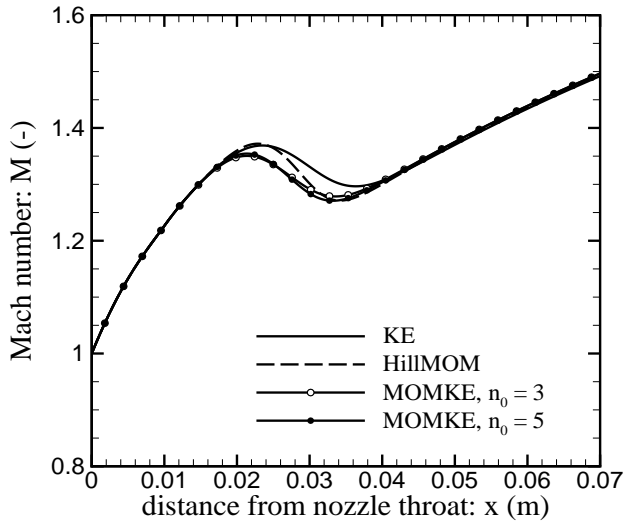

(b)

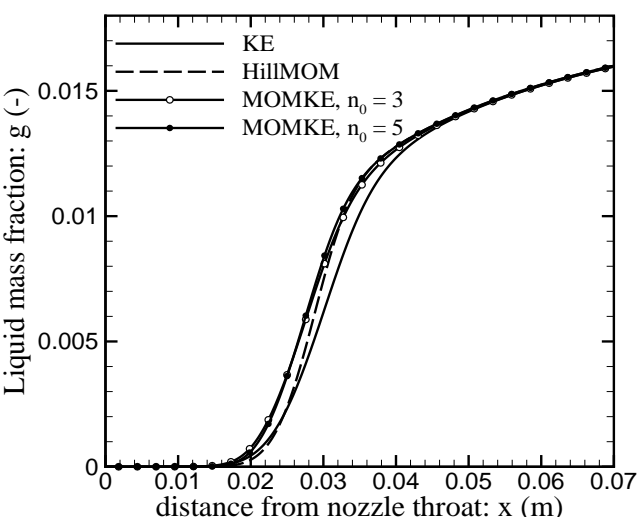

(d)

FIGURE 4.2: Flow field and thermodynamic variables for test case 2, predicted with Hill's MOM for $\mu_{k}$ (denoted as 'HillMOM, GDE' in the figure, see also Eqs. (4.21) and (4.39)), or Hill's MOM for $\bar{v}_{k}$ (denoted as 'MOMKE' in the figure, see also Eq. (4.11)), along with the benchmark solution from a FDE/KE-simulation. $n_{0}$ denotes the lower boundary for the moments $\bar{v}_{k}$.

ratio shows differences of up to $60 \%$ in the nucleation zone.

\subsection{Conclusions}

Reduced models based on the moments of the droplet size distribution, have been evaluated for application to condensing flow. In addition to Hill's method of moments derived from the general dynamic equation (GDE), a novel set of moment equations, derived from the kinetic equation (KE) has been presented. 


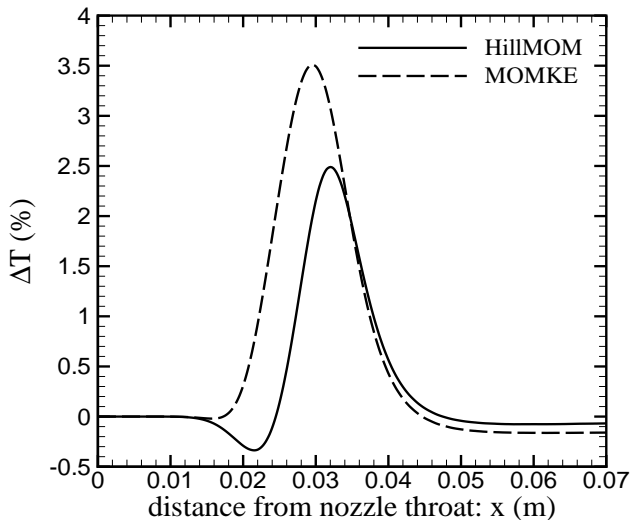

(a)

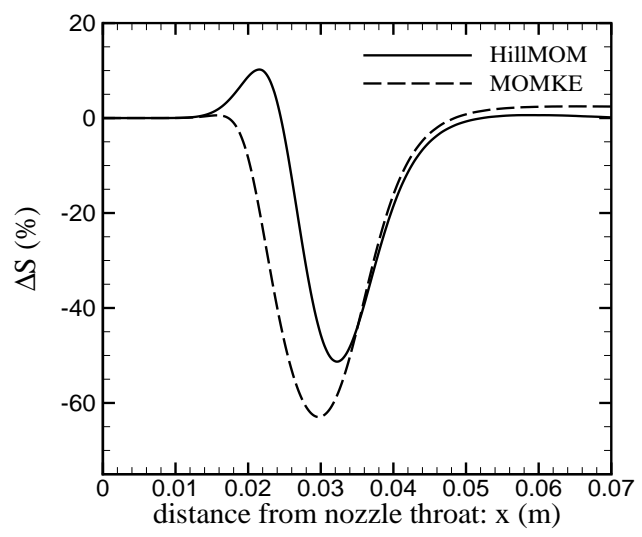

(c)

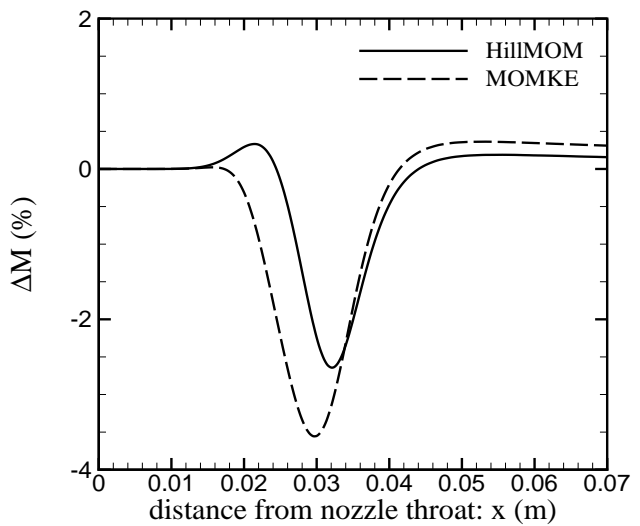

(b)

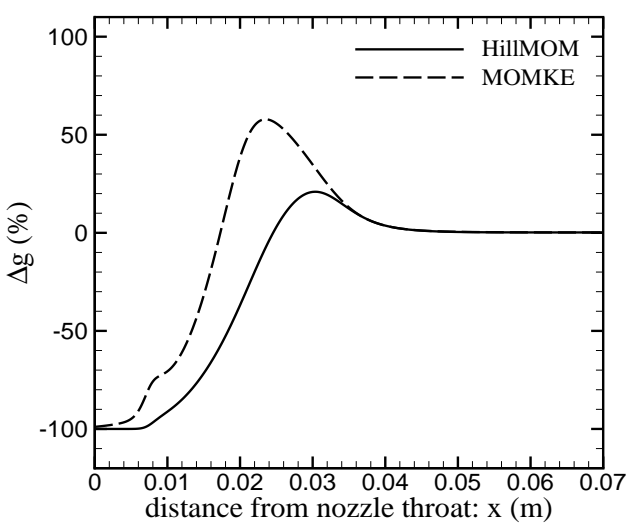

(d)

FIGURE 4.3: Relative differences between flow field and thermodynamic variables for test case 2, corresponding with the data in Figures 4.4.a-d. 'HillMOM' is associated with the MOM for $\mu_{k}$, wheras 'MOMKE' is associated with the MOM for $\bar{v}_{k}$, with $n_{0}=5$.

Each variant of the method of moments (MOM) has two associated closure problems: (i) one representing a sum, or an integral over droplet size space, and (ii) another one which requires the local solution of the droplet size distribution (DSD) at a single droplet size. Two quadrature based methods (QMOM and DQMOM) are evaluated for closure of the first problem, whereas the second one remains unresolved. Analysis reveals that both QMOM and DQMOM fail to reproduce the steady state and equilibrium behavior of the KE, with both additionally suffering from a lack of robustness.

The accuracy of the MOM is finally quantified by using a condensing nozzle flow 


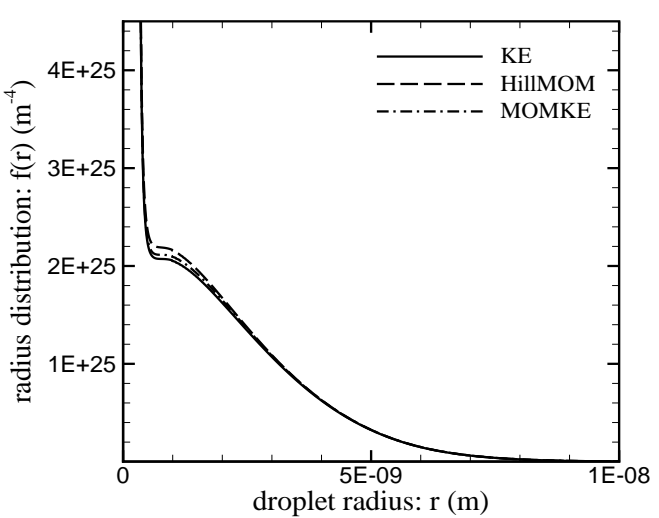

(a)

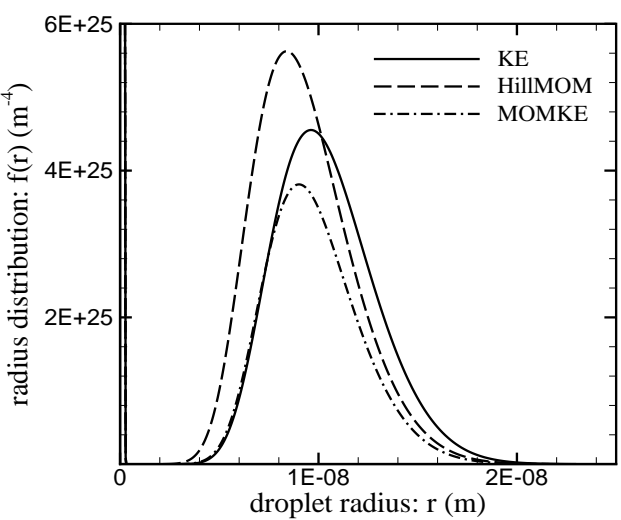

(c)

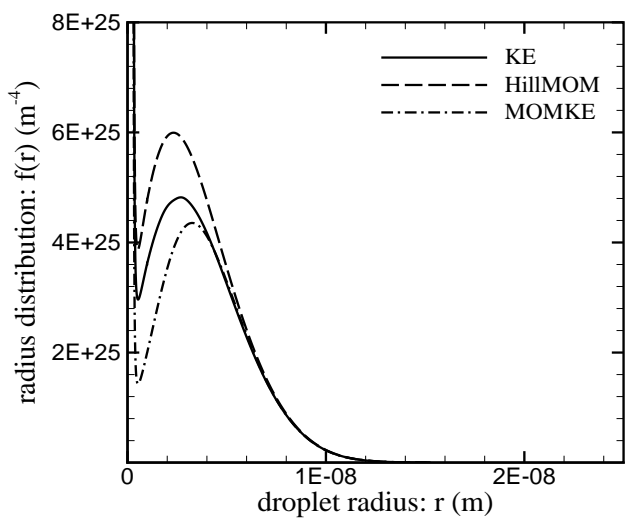

(b)

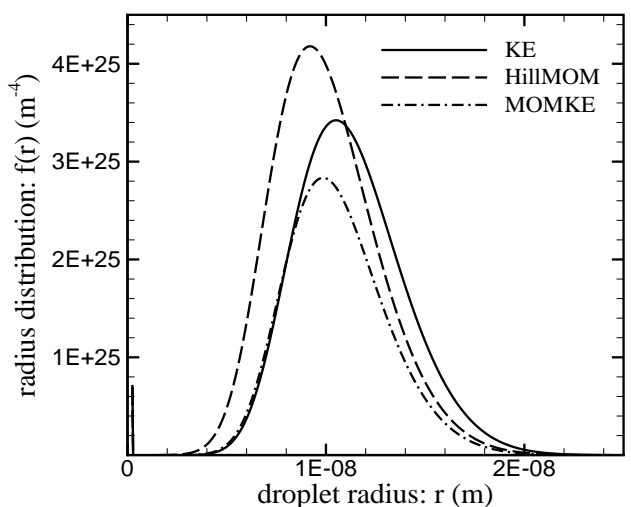

(d)

FIGURE 4.4: Droplet radius distribution function $f(r)$ at four different positions along the nozzle: (a) $x=0.016 m$ (start of nucleation zone), (b) $x=0.022 m$, (c) $x=$ $0.042 \mathrm{~m}$ (end of nucleation zone), and (d) $x=0.070 \mathrm{~m}$ (nozzle exit). ' $K E$ ' denotes the $D S D$ from a KE/FDE-solution, 'HillMOM' refers to the DSD that is reconstructed by postprocessing flow field data predicted with Hill's MOM, and 'MOMKE' refers to the DSD that is reconstructed by postprocessing flow field data predicted with the MOM for $\bar{v}_{k}$, with $n_{0}=5$.

experiment for which benchmark KE-solutions have been computed. Comparison of predicted flow field variables reveals that the MOM is capable of encompassing condensation effects with reasonable accuracy. Moreover, the DSD reconstructed from flow field data predicted with the MOM compare favorably with solutions computed directly from the KE and FDE. 


\section{EVALUATION OF A FLOW MODEL WITH BINARY CONDENSATION}

In this chapter, an evaluation is made of a condensing flow model, which utilizes a kinetic equation to predict the evolution of a two-component (binary) dispersion, generated by homogeneous nucleation. The evaluation is based on a simulation of a nozzle flow experiment, for which measurement data are reported in the literature. The application of a full two-way coupling between the thermodynamic variables and the binary droplet size distribution has not been done before. Comparison of predicted and measured temperatures and partial pressures at the onset of condensation shows a fair agreement between theory and experiment. The full resolution of the binary droplet size distribution is exploited to verify whether or not a quasisteady treatment of the nucleation process is warranted. Finally, the sensitivity of the condensation model with respect to the binary equilibrium distribution is examined.

\subsection{Introduction}

Multi-component condensation is an area of research which is of fundamental interest to applications in nature and industry, e.g., in the formation of cloud condensation nuclei [101], or the conditioning of natural gas [91], [92]. Throughout the years, various condensation models have been developed, which focus on specific applications. The modelling approach in this investigation is tailored to condensation in rapid expansions, where the kinetically driven process of homogeneous nucleation is responsible for the conception of condensation nuclei.

The success of the kinetic model pioneered by a.o. Becker and Döring [13] for unary (single component) condensation, logically prompted the extension towards multi-component systems. Having reviewed the uncertainties associated with the unary kinetic equation (KE) in chapter 3 , it can be stated that these also carry over to the multi-component case. The composition of multi-component droplets leads to additional uncertainties in physical modelling, therefore, most investigations have been limited to binary (two-component) condensation.

The theoretical treatment of binary condensation is primarily focused on steady- 
state nucleation, where the aim is to predict the steady-state nucleation rate in twodimensional component space. The first treatise on binary (or heteromolecular) nucleation was conceived by Flood [32], who predicted an exponential dependence of the binary nucleation rate $(J)$ on the critical Gibbs free energy of droplet formation $\left(\Delta G^{*}\right)$, i.e., $J \sim \exp \left(-\Delta G^{*} / k_{B} T\right)$, with $T$ the temperature and $k_{B}$ the Boltzmann constant. This is similar to the generic expression for the nucleation rate for the unary case. The first exposition on the actual kinetics of binary condensation was given by Reiss in [97]. In this seminal work, the binary kinetic equation (BKE) for the two-component droplet size distribution is presented, and subsequently, its steadystate form is utilized to derive expressions for both the magnitude and orientation of the steady-state nucleation rate vector. The theory developed by Reiss suffers mainly from inconsistencies associated with the equilibrium binary droplet size distribution, which is used to calculate the evaporation (or backward) rate of droplets. These inconsistencies have been examined in various previous investigations, and a number of attempts have been made to derive more sound expressions, notably in [56], and [135].

Besides the inconsistencies associated with the equilibrium distribution, there are also a number of inaccuracies related to the derivation of the expressions for the steady-state nucleation rate in [97]. The cardinal assumption in this theory is that the maximum nucleation flux follows the path corresponding with the smallest energy barrier through the saddle-point of the binary Gibbs free energy-plane. An extensive analysis by Stauffer in [114] has revealed that this steepest descent approach is generally not valid, although for a number of systems, it does appear to be a good approximation. Both Reiss and Stauffer utilize the continuum approximation of the BKE in their derivation of the steady-state nucleation rate. The inherent assumption made is that droplets are so large that a single monomer difference between successive droplet sizes can be regarded as an infinitesimal variation, which allows the discrete BKE to be replaced by a continuous Fokker-Planck type of partial differential equation in binary size space [30].

The latest trend in binary nucleation theory is to use numerical techniques to solve the steady-state form of the BKE directly, without resorting to continuum approximations, such as demonstrated in [74], and [126]. This allows for a more comprehensive comparison with experiments, as the steady-state droplet size distribution is resolved in full detail. With the advent of new measurement techniques that utilize condensing nozzle flows [144], [44], it is very likely that the rapid variations in vapor supersaturation and temperature do not allow for application of quasisteady-state nucleation theory. Under such conditions, it is necessary to incorporate the effects of vapor depletion and latent heat release on the flow variables, which necessitates the simultaneous solution of the fluid dynamics equations (FDE) and the BKE.

In this chapter a fully two-way coupled model is presented for inviscid compress- 
ible flow with binary condensation. The accuracy of the model is evaluated by comparing the predicted temperatures and pressures at condensation onset with measurement data taken from literature [144]. By using a sectional method, the BKE is fully resolved beyond the nanometer size scale, in order to quantify the latent heat release and vapor depletion effect with sufficient accuracy. The numerical method is first tested utilizing one-way coupled test cases reported in literature, whereafter the results obtained with a two-way coupled simulation of a nozzle flow experiment are analyzed. Subsequently, the fully resolved solution of the BKE is analyzed to determine the validity of the quasisteady-state approximation. Finally, a sensitivity analysis is carried out to quantify the influence of the binary equilibrium size distribution on the flow field variables.

\subsection{The kinetic equation for the binary size distribution}

Similar to the unary KE, the BKE is a mass conservation law for droplets in binary size space. Assuming the Szilard model for condensation, i.e., droplets may only capture or expel a single monomer of either type at any instant, the BKE for an advected dispersion can be written as:

$$
\frac{\partial \hat{\rho}_{\mathbf{n}}}{\partial t}+\frac{\partial}{\partial x_{j}}\left(\hat{\rho}_{\mathbf{n}} u_{j}\right)=J_{\mathbf{n}-\mathbf{e}_{1}, 1}-J_{\mathbf{n}, 1}+J_{\mathbf{n}-\mathbf{e}_{2}, 2}-J_{\mathbf{n}, 2},
$$

where $\mathbf{n}=\left(n_{1}, n_{2}\right)^{T}$ is the coordinate in binary space, $\mathbf{e}_{1}=(1,0)^{T}, \mathbf{e}_{2}=(0,1)^{T}, \hat{\rho}_{\mathbf{n}}$ is the volumetric concentration of droplets consisting of $n_{1}$ monomers of component 1 , and $n_{2}$ monomers of component 2 , and where $J_{\mathbf{n}, k}$ denotes the nett rate at which clusters of size $\mathbf{n}$ grow to size $\mathbf{n}+\mathbf{e}_{k}$. Schematically, the BKE can be represented as a series of chemical reactions, such as depicted in Figure 5.1. It is noted that creation of the binary dimer $\mathbf{n}=(1,1)^{T}$ from two monomers $(1,0)^{T}$ and $(0,1)^{T}$ constitutes a single reaction, which means that (i) the monomer fluxes $J_{(1,0), 2}$ and $J_{(0,1), 1}$ are equal, and (ii) that only one of these should be used to compute the residuals for $\hat{\rho}_{(1,1)}$.

The condensation flux $J_{\mathbf{n}, k}$ comprises the nett effect of condensation and evaporation, via corresponding forward rates $f_{\mathbf{n}, k}$ and backward rates $b_{\mathbf{n}, k}$, respectively:

$$
J_{\mathbf{n}, k}=f_{\mathbf{n}, k} \hat{\rho}_{\mathbf{n}}-b_{\mathbf{n}+\mathbf{e}_{k}, k} \hat{\rho}_{\mathbf{n}+\mathbf{e}_{k}} .
$$

\section{Forward and backward rates}

Assuming Maxwellian velocity distributions for the monomers and the droplets, the forward rate $f_{\mathbf{n}, k}$ for the $k^{\text {th }}$-component is given by [127]:

$$
f_{\mathbf{n}, k}=\alpha_{\mathbf{n}} c_{\mathbf{n}} \frac{\rho_{v, k}}{m_{v, k}} \sqrt{6 k_{B} T} \sqrt{\frac{1}{m_{\mathbf{n}}}+\frac{1}{m_{v, k}}}\left(\frac{3}{4 \pi}\right)^{1 / 6}\left(v_{\mathbf{n}}^{1 / 3}+v_{v, k}^{1 / 3}\right)^{2},
$$




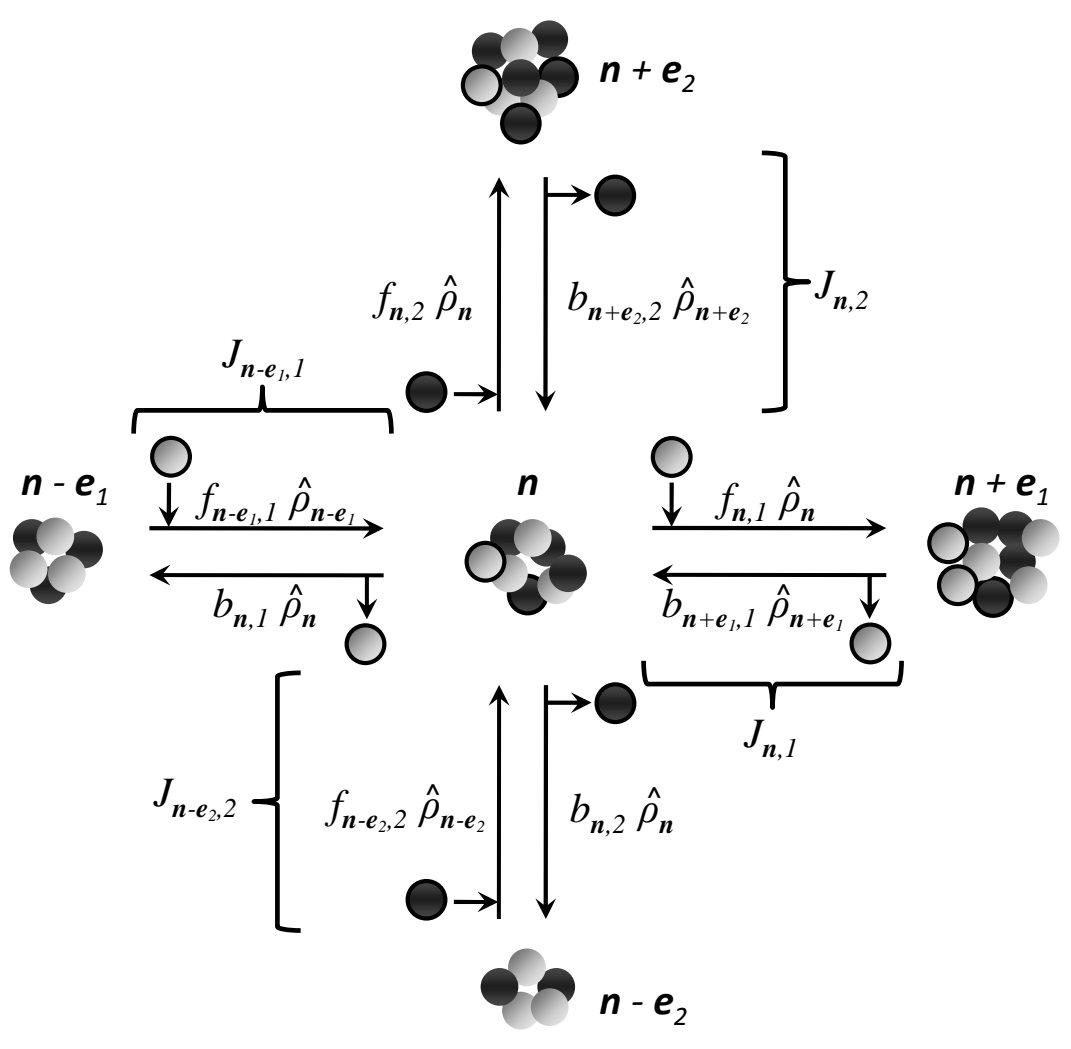

FIGURE 5.1: Schematic representation of the Szilard model for binary condensation.

where $\alpha_{\mathbf{n}}, c_{\mathbf{n}}, m_{\mathbf{n}}$, and $v_{\mathbf{n}}$ are the sticking probability, shape factor, mass, and volume of a cluster of size $\mathbf{n}$, respectively. The mass and volume associated with a monomer of component $k$ are denoted by $m_{v, k}$ and $v_{v, k}$, respectively. The same uncertainties regarding the sticking probability and the shape factor for the unary case (see chapter 3 ) are present in the binary case, and therefore, these will be exempted from further discussion in this chapter. The sticking probability will be set to constant value of $\alpha_{\mathbf{n}}=1$, and a spherical droplet shape will be assumed $\left(c_{\mathbf{n}}=1\right)$, as is common practice in the nucleation literature.

The backward rate $b_{\mathbf{n}, k}$ for component $k$ is calculated from the corresponding forward rate and the equilibrium droplet size distribution $\hat{\rho}_{\mathbf{n}}^{e q}$, by virtue of the principle of detailed balance, [127]:

$$
b_{\mathbf{n}, k}=f_{\mathbf{n}-\mathbf{e}_{k}, k} \frac{\hat{\rho}_{\mathbf{n}-\mathbf{e}_{k}}^{e q}}{\hat{\rho}_{\mathbf{n}}^{e q}} .
$$




\section{Binary equilibrium size distributions}

The binary equilibrium distribution $\hat{\rho}_{\mathbf{n}}^{e q}$ has been the subject of many discussions in the nucleation literature, e.g., in [135], [56], and [31]. The generic expression for $\hat{\rho}_{\mathbf{n}}^{e q}$ is given as [127]:

$$
\hat{\rho}_{\mathbf{n}}^{e q}=\hat{\rho}_{0} \exp \left(-\frac{\Delta G_{\mathbf{n}}}{k_{B} T}\right),
$$

where $\Delta G_{\mathbf{n}}$ is the Gibbs free energy associated with a droplet of size $\mathbf{n}$, and $\hat{\rho}_{0}$ is a pre-factor which determines the magnitude of the equilibrium number densities.

Most models for $\Delta G_{\mathbf{n}}$ reported in the literature, utilize the following expression [135], [127], [71]:

$$
\Delta G_{\mathbf{n}}=-k_{B} T\left(n_{1} \ln \frac{a_{v, 1}}{a_{\mathbf{n}, 1}}+n_{2} \ln \frac{a_{v, 2}}{a_{\mathbf{n}, 2}}\right)+A_{\mathbf{n}} \sigma_{\mathbf{n}},
$$

where $a_{v, k}$ is the vapor activity of component $k$ in the vapor phase, $a_{\mathbf{n}, k}$ is the liquid activity of vapor component $k$ in an $\mathbf{n}$-droplet, and where $A_{\mathbf{n}}$ and $\sigma_{\mathbf{n}}$ denote the surface area and surface tension of an $\mathbf{n}$-droplet, respectively. The activities of the vapor and liquid phases are defined as:

$$
a_{v, k} \equiv \frac{p_{v, k}}{p_{v, k}^{s, p}}, \text { and }: \quad a_{\mathbf{n}, k} \equiv \frac{p_{v, k}^{s}}{p_{v, k}^{s, p}},
$$

respectively, where $p_{v, k}$ is the partial vapor pressure of component $k, p_{v, k}^{s, p}=p_{v, k}^{s, p}(T)$ is the pure-component saturation pressure of vapor component $k$ over a flat liquid surface, and where $p_{v, k}^{s}=p_{v, k}^{s}(\mathbf{n}, T)$ is the partial saturation pressure for vapor component $k$ over the surface of an $\mathbf{n}$-droplet. The liquid activity is usually expressed as

$$
a_{\mathbf{n}, k}=\gamma_{\mathbf{n}, k} x_{\mathbf{n}, k}
$$

where $\gamma_{\mathbf{n}, k}=\gamma_{\mathbf{n}, k}(T)$ is the so-called activity coefficient, and $x_{\mathbf{n}, k}$ denotes the molar fraction of vapor component $k$ in the droplet,

$$
x_{\mathbf{n}, k}=\frac{n_{k}}{n_{1}+n_{2}} .
$$

In analogy with unary condensation, a component saturation ratio $S_{v, k}$ can also be introduced,

$$
S_{v, k}=S_{v, k}\left(\mathbf{n}, T, p_{v, k}\right) \equiv \frac{p_{v, k}}{p_{v, k}^{s}(\mathbf{n}, T)}=\frac{a_{v, k}}{a_{\mathbf{n}, k}},
$$

so that $\Delta G_{\mathbf{n}}$ can be expressed in more familiar form as:

$$
\Delta G_{\mathbf{n}}=-k_{B} T\left(n_{1} \ln S_{v, 1}+n_{2} \ln S_{v, 2}\right)+A_{\mathbf{n}} \sigma_{\mathbf{n}} .
$$


For the pre-factor $\hat{\rho}_{0}$ in Eq. (5.5), the variation in available models is more diverse. Since the pre-factor can also be a function of the droplet size, it may exert a profound influence on the evolution of the droplet size distribution via the backward rate expression, viz. Eq. (5.4). In view of its anticipated significance, the following variants for $\hat{\rho}_{0}$ are considered in the present investigation:

1. $\hat{\rho}_{0}$ for the classical equilibrium distribution by Reiss, [97]:

$$
\hat{\rho}_{0}^{R}=\hat{\rho}_{(1,0)}+\hat{\rho}_{(0,1)},
$$

2. $\hat{\rho}_{0}$ for the equilibrium distribution with limited self-consistency, by Wilemski and Wyslouzil , [134]:

$$
\hat{\rho}_{0}^{W W 1}=\left(\frac{\rho_{v, 1}^{s, p}}{m_{v, 1}}\right)^{x_{\mathbf{n}, 1}}\left(\frac{\rho_{v, 2}^{s, p}}{m_{v, 2}}\right)^{x_{\mathbf{n}, 2}},
$$

3. $\hat{\rho}_{0}$ for the equilibrium distribution with full consistency, by Wilemski and Wyslouzil, [134]:

$$
\hat{\rho}_{0}^{W W 2}=\hat{\rho}_{0}^{W W 1} \exp \left(x_{\mathbf{n}, 1} \theta_{v, 1}+x_{\mathbf{n}, 2} \theta_{v, 2}\right),
$$

where $\rho_{v, k}^{s, p}$ is the mass density of pure vapor component $k$ at saturation, and where the dimensionless surface energy $\theta_{v, k}$ is given by:

$$
\theta_{v, k}=\frac{A_{v, k} \sigma_{v, k}}{k_{B} T}
$$

with $A_{v, k}$ the effective surface area of a monomer of component $k$, and $\sigma_{v, k}$ the surface tension of a flat liquid interface of pure component $k$.

It is noted that each of the three equilibrium distribution models is based on the capillarity approach, which treats a droplet as a macroscopic entity. As noted by Wilemski and Wyslouzil [135], the use of such models constitutes a pragmatic approach, which is necessarily maintained until a sound molecular theory becomes available.

In calculating $\Delta G_{\mathbf{n}}$, the effect of both droplet curvature and composition should ideally be taken into account when specifying the liquid phase activities and surface tension for an $\mathbf{n}$-droplet. It is important to note that the droplet composition is generally not uniformly distributed within the droplet. The consensus within the nucleation community is that a droplet can be modelled as consisting of a bulk internal phase and a surface phase, for which the respective compositions $x_{b, k}$ and $x_{s, k}$ can be introduced [127]. Due to the phenomenon of surface enrichment, the surface composition is usually different from the bulk composition, especially for non-ideal systems such as aqueous alcohols [71]. Moreover, the bulk composition $x_{b, k}$ can also differ significantly from the total composition $x_{\mathbf{n}, k}$ (see Eq. (5.9)), which means that 
when calculating the liquid phase activities and surface tension based on empirical expressions, one should use the bulk composition and not the total composition. Fortunately, the surface tension of the binary systems studied in this investigation do not vary appreciably with molar composition, and consequently only a small error is made when $x_{\mathbf{n}, k}$ (Eq. (5.9)) is used instead of the bulk composition $x_{b, k}$. In principle, it is possible to incorporate surface enrichment effects into the model, but this will not be attempted here because available models which take surface enrichment into account are not void of unphysical behavior, as illustrated in [57] and [127]. Furthermore, incorporation of surface enrichment is not desired from a computational point of view, as determination of the surface composition usually proceeds in an iterative fashion (see, e.g., [127]), which can significantly increase computational expenses.

The equilibrium size distributions given by Eq. (5.5), Eq. (5.11), with the pre-factor $\hat{\rho}_{0}$ given by either Eq. (5.12), (5.13), or (5.14), should satisfy the following consistency conditions:

1. the law of mass action needs to be satisfied, i.e., substitution of $\hat{\rho}_{\mathbf{n}}^{e q}$ in Eq. (5.4) should yield a backward rate that is independent of the monomer concentrations.

2. the binary equilibrium distribution should reduce to the appropriate unary equilibrium distribution when only one component is present (i.e., either $n_{1}=0$, or $\left.n_{2}=0\right)$.

3. the monomer concentrations of each component should be retrieved from the equilibrium distributions upon substitution of the unary size $\mathbf{n}=\mathbf{e}_{k}$.

If all three conditions are satisfied, the equilibrium size distribution is said to be fully self-consistent, otherwise it is said to have limited self-consistency. The following observations can be made regarding the preceding equilibrium size distributions:

- the Reiss distribution does not satisfy any of the three conditions;

- both distributions by Wilemski and Wyslouzil satisfy the first and last condition, whereas the second condition is only satisfied by $\hat{\rho}_{\mathbf{n}}^{e q, W W 2}$.

Given that $\hat{\rho}_{\mathbf{n}}^{e q, R}$ does not satisfy the law of mass action, this equilibrium distribution will not be considered any further in the present investigation. Even though the WW1-equilibrium distribution has limited self-consistency, it will be employed here along with the WW2-equilibrium distribution, in order to quantify the influence of the equilibrium size distribution on the flow field solution. 


\subsection{Mixture properties and fluid dynamics equations}

\section{Mixture properties}

The binary mixtures employed in this investigation can be approximated as ideal systems, both in the gaseous and liquid states. Therefore, the thermal and caloric equations of state as derived in chapter 2 are valid for the test cases considered here.

The thermodynamic model needs to be augmented with suitable expressions for the saturation vapor densities $\rho_{v, k}^{s}$, and the surface tension $\sigma_{\mathbf{n}}$. Both the liquid mixture in the droplets and the vapor mixture in the gas phase are assumed to be ideal, which means that the liquid phase activity coefficients are unity: $\gamma_{\mathbf{n}, k}=1$. Neglecting droplet curvature, the partial saturation pressure $p_{v, k}^{s}(\mathbf{n}, T)$ of vapor component $k$ over the surface of an $\mathbf{n}$-droplet is given by [103]:

$$
p_{v, k}^{s}(\mathbf{n}, T)=x_{\mathbf{n}, k} p_{v, k}^{s, p}(T) .
$$

Using the thermal equation of state, the saturation density then follows as:

$$
\rho_{v, k}^{s}\left(x_{\mathbf{n}, k}\right)=\frac{p_{v, k}^{s}}{R_{v, k} T}
$$

For the surface tension of the liquid mixture, a linear weighing of the pure-component surface tensions in terms of the molar fractions will be employed, i.e.,

$$
\sigma_{\mathbf{n}}=x_{\mathbf{n}, 1} \sigma_{v, 1}+\left(1-x_{\mathbf{n}, 1}\right) \sigma_{v, 2}
$$

with $x_{\mathbf{n}, k}$ given by Eq. (5.9).

The latent heat of condensation $\left(L_{\mathbf{n}}\right.$, see, e.g., Eq. (2.130)) for a binary droplet, is calculated in similar fashion, i.e.,

$$
L_{\mathbf{n}}=w_{\mathbf{n}, 1} L_{b, 1}+\left(1-w_{\mathbf{n}, 2}\right) L_{b, 2},
$$

where $L_{b, k}$ is the bulk latent heat of pure vapor component $k$, and $w_{\mathbf{n}, k}$ is the mass fraction of component $k$ in the droplet (see Eq. (2.118)).

\section{Fluid dynamics equations}

The conservation form of the FDE for binary condensation has been presented in chapter 2. Due to the relatively large number of degrees of freedom associated with the BKE, it is computationally convenient to employ a so-called space marching solution method here, instead of the standard finite-volume discretization used in chapter 3. Furthermore, a quasi-one dimensional approximation is employed for the nozzle flow, since the area variation depicted in Figure 5.2 implicates a slender nozzle geometry. 
The unknown variables at each point along the nozzle include the temperature and vapor densities, as well as the binary number densities $\hat{\rho}_{\mathbf{n}}$. Instead of $\hat{\rho}_{\mathbf{n}}$, the specific number density $\check{\rho}_{\mathbf{n}} \equiv \hat{\rho}_{\mathbf{n}} / \rho$ will be employed. By combining the continuity equation for the mixture (Eq. (2.84)) with the BKE (Eq. (5.1)), the steady-state Lagrangian form of the BKE is obtained:

$$
\frac{d \check{\rho}_{\mathbf{n}}}{d x}=\frac{\check{S}_{\mathbf{n}}}{u},
$$

where $x$ is the co-ordinate along the nozzle axis, $u$ the axial velocity, and where $\breve{S}_{\mathbf{n}}=S_{\mathbf{n}} / \rho$, with $S_{\mathbf{n}}$ the right-hand side of Eq. (5.1).

Due to the assumed steady-state character of the nozzle flow, the mass flow rate $\phi_{m}$ of the mixture is constant, which means that at each position $x$ :

$$
\rho u A=\phi_{m},
$$

where $A=A(x)$ is the cross-sectional area of the nozzle at position $x$. By assuming choked flow, and that the onset of condensation takes place downstream of the throat, the mass flow rate can be obtained from isentropic flow theory, thus:

$$
\phi_{m}=\rho_{0} \sqrt{\gamma_{m, 0} R_{m, 0} T_{0}}\left[1+\frac{1}{2}\left(\gamma_{m, 0}-1\right)\right]^{\frac{1+\gamma_{m, 0}}{2\left(1-\gamma_{m, 0}\right)}} A_{t},
$$

where $A_{t}$ is the flow area at the nozzle throat, $T_{0}$ and $\rho_{0}$ are the total temperature and density of the dry mixture, respectively, and where:

$$
\gamma_{m, 0}=\frac{C_{p, 0}}{C_{v, 0}}, R_{m, 0}=C_{p, 0}-C_{v, 0},
$$

are the Poisson constant, and specific gas constant of the dry mixture, respectively. Differentiation of Eq. (5.21) with respect to $x$ yields the following quasi-one-dimensional and steady-state differential form of the continuity equation for the mixture:

$$
\frac{1}{u} \frac{d u}{d x}+\frac{1}{\rho} \frac{d \rho}{d x}+\frac{1}{A} \frac{d A}{d x}=0 .
$$

In the absence of viscous dissipation and heat conduction, the total enthalpy $h_{t}$ of the mixture remains invariant along streamlines. Using Eqs. (2.128) and (5.19), the total mixture enthalpy is expressed as:

$$
h_{t}=C_{p, 0} T-\left[g_{l, 1} L_{b, 1}(T)+g_{l, 2} L_{b, 2}(T)\right]+\frac{1}{2} u^{2},
$$

where the liquid mass fraction $g_{l, k}$ of vapor component $k$ is given by Eq. (2.119). It is noted that $g_{l, k}$ is completely determined by the number densities $\check{\boldsymbol{\rho}}=\left(\check{\rho}_{(2,0)}, \check{\rho}_{(0,2)}, \ldots, \check{\rho}_{(N, N)}\right)^{T}$. Differentiation of Eq. (5.24) with respect to $x$ thus yields:

$$
\left[C_{p, 0}-\left(g_{l, 1} \frac{d L_{b, 1}}{d T}+g_{l, 2} \frac{L_{b, 2}}{d T}\right)\right] \frac{d T}{d x}-\frac{d g_{l, 1}}{d x} L_{b, 1}-\frac{d g_{l, 2}}{d x} L_{b, 2}+u \frac{d u}{d x}=0 .
$$


Due to the inviscid modelling approach, the momentum equation for the mixture is reduced to:

$$
u \frac{d u}{d x}+\frac{1}{\rho} \frac{d p}{d x}=0
$$

for a quasi-one-dimensional system.

Eqs. (5.20), (5.23), (5.25), and (5.26) constitute the governing system of equations which describe the condensing flow in a quasi-one-dimensional geometry, in terms of the state variables $\rho, \check{\rho}, T, u$, and $p$. To complete the system, the thermal equation of state:

$$
p=\rho R T
$$

is also included, with the specific mixture gas constant $R=R\left(g_{l, 1}, g_{l, 2}\right)$ given by Eq. (2.122). Differentiation with respect to $x$ yields:

$$
\frac{1}{p} \frac{d p}{d x}=\frac{1}{\rho} \frac{d \rho}{d x}+\frac{1}{R} \frac{d R}{d x}+\frac{1}{T} \frac{d T}{d x}
$$

where:

$$
\frac{d R}{d x}=\frac{\partial R}{\partial g_{l, 1}} \frac{d g_{l, 1}}{d x}+\frac{\partial R}{\partial g_{l, 2}} \frac{d g_{l, 2}}{d x} .
$$

By combining Eqs. (5.23), (5.25), (5.26), and (5.27), the following expression for the spatial derivative of the temperature can be derived:

$$
\frac{d T}{d x}=\frac{\frac{d g_{l, 1}}{d x} L_{b, 1}+\frac{d g_{l, 2}}{d x} L_{b, 2}-\frac{u^{2}}{1-\frac{u^{2}}{R T}}\left[\frac{1}{R} \frac{d R}{d x}-\frac{1}{A} \frac{d A}{d x}\right]}{C_{p, 0}-\left[g_{l, 1} \frac{d L_{b, 1}}{d T}+g_{l, 2} \frac{L_{b, 2}}{d T}\right]+\frac{u^{2} / T}{1-\frac{u^{2}}{R T}}} .
$$

The governing system of equations can now be reduced to Eq. (5.20) for $\check{\rho}$, and Eq. (5.29) for $T$. Knowing $\check{\boldsymbol{\rho}}$, the liquid mass fractions $g_{l, 1}$ and $g_{l, 2}$ can be calculated by means of Eq. (2.119). As the total enthalpy $h_{t}$ is invariant, the fluid velocity can be calculated from the temperature and the liquid mass fractions, via Eq. (5.24). Finally, the fluid velocity and flow area $A(x)$ can be used to compute the mixture density via Eq. (5.21).

It is noted that the calculation strategy of using Eq. (5.20) and Eq. (5.29) in a space marching method can only be applied when the flow remains supersonic in the divergent part of the nozzle. This restricts the applicability of the condensing flow model to gradually expanding (i.e., shock-free) nozzle flows. 


\subsection{Evaluation procedure}

\subsubsection{Description of test cases}

Two test cases will be considered in this investigation: the first one is a simulated nucleation experiment, and the second one a condensing nozzle flow.

\section{Test case 1: nucleation simulation}

The first test case is utilized for verification of the numerical algorithm which solves the BKE. A one-way coupled simulation is carried out, i.e., $u=0$ and the mixture state variables $\rho, T$, and $\rho_{v, k}$ are frozen. The initial condition for the binary size distribution is a zero-distribution, i.e., $\hat{\rho}_{\mathbf{n}}=0$ for $\|\mathbf{n}\|>1$. The binary system considered is a mixture of ethanol and hexanol vapor, which are assigned the component indices 1 and 2, respectively. The relevant material properties are given in appendix A. Verification of the numerical code is carried out by comparing the predicted transient and quasisteady-state binary size distributions with the results published in [142] and [143]. For each of the simulations related to the first test case, the temperature is maintained at a constant value of $T=260 \mathrm{~K}$, whereas three different pairs of vapor activities are considered: (i) $a_{v, 1}=0.5$ and $a_{v, 2}=14.0$ (case 1.1), $a_{v, 1}=1.5$ and $a_{v, 2}=9.0$ (case 1.2), (iii) $a_{v, 1}=2.5$ and $a_{v, 2}=6.0$ (case 1.3). By default, the backward rates in test cases 1.1-1.3 are computed by using the WW2-equilibrium distribution.

\section{Test case 2: condensing nozzle flow}

In the second test case, a condensing nozzle flow is simulated for which measurement data is reported in [144]. The mixture contains ethanol- and propanol vapor as the condensable components, with nitrogen as the carrier gas. The nozzle area variation, depicted in Figure 5.2, is reconstructed from the dry-flow pressure profiles given in [144] by means of the isentropic relationships for quasi-one-dimensional compressible flow. This approach is adopted, because the measured pressure profiles account for the presence of boundary-layers in the experiments. The total conditions at the nozzle inlet are maintained at $T_{0}=286.15 \mathrm{~K}$, and $p_{0}=59.1 \mathrm{kPa}$, and the mixture compositions are given in table 5.1. The relevant fluid properties for the various components are given in appendix A.

In the simulation for each test case, a full two-way coupling is employed, meaning that Eq. (5.20) for $\check{\rho}$, and Eq. (5.29) for $T$ are simultaneously solved throughout the nozzle. Unless specified otherwise, the backward rate is computed by using the WW2-equilibrium distribution. 


\begin{tabular}{lcccc}
\hline \hline case no.: & $y_{v, 1}(-)$ & $y_{v, 2}(-)$ & $g_{v, 1}\left(\times 10^{-3}\right)$ & $g_{v, 2}\left(\times 10^{-3}\right)$ \\
\hline $2.1(100 \%$ ethanol $)$ & 1.00 & 0.00 & 4.379 & 0.0 \\
$2.2(75 \%$ ethanol $)$ & 0.75 & 0.25 & 2.592 & 1.127 \\
$2.3(50 \%$ ethanol $)$ & 0.50 & 0.50 & 1.524 & 1.992 \\
$2.4(25 \%$ ethanol $)$ & 0.25 & 0.75 & 7.366 & 2.886 \\
$2.5(0 \%$ ethanol $)$ & 0.00 & 1.00 & 0.0 & 2.931 \\
\hline \hline
\end{tabular}

TABLE 5.1: Molar composition of the vapor phase and mixture mass fractions for the ethanol-propanol mixtures studied in the second test case. Note that unary condensation takes place in test cases 2.1 and 2.5.

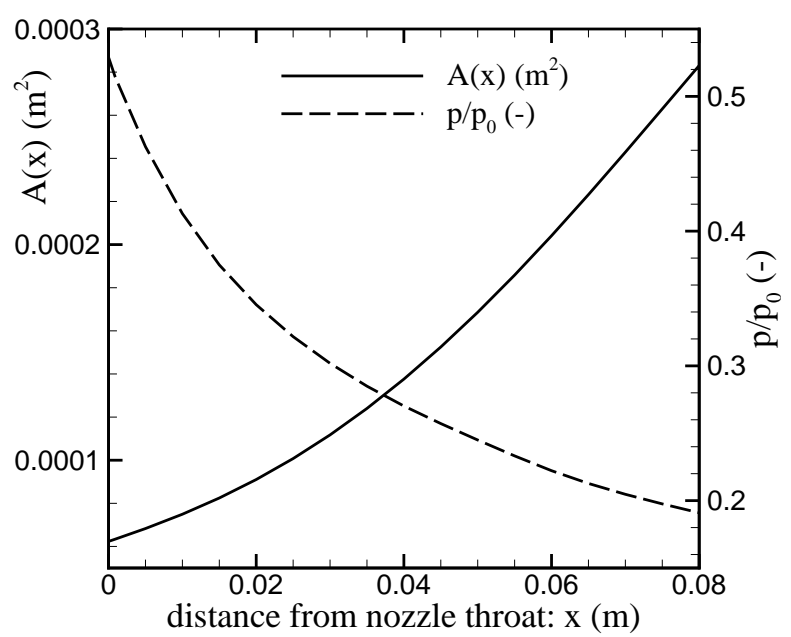

FIGURE 5.2: Reconstructed nozzle profile $A(x)$ and dimensionless pressure profile for a dry flow of $\mathrm{N}_{2}$ from [144], used for test cases 2.1 - 2.5.

\subsubsection{Numerical methods}

For the stagnant systems in the first test case, Heun's predictor-corrector method is used to march the BKE in time. The maximum droplet size is set to $N_{1} \times N_{2}=$ $100 \times 100$, whereas the lower boundary is taken at the unary droplets consisting of either vapor component. Furthermore, the smallest clusters are the unary dimers $\left(\mathbf{n}=(2,0)^{T}\right.$, or $\left.(0,2)^{T}\right)$, and the binary dimer $\left(\mathbf{n}=(1,1)^{T}\right)$, as depicted in Figure 5.3. The boundary conditions for the BKE are prescribed via the vapor monomer concentrations $\hat{\rho}_{\mathbf{e}_{k}}=\rho_{v, k} / m_{v, k}$ at the lower boundary, whereas the number densities for the largest droplets are extrapolated from the interior of the computational domain. The method described here is actually similar to the one used by Wyslouzil and Wilemski in [142] and [143]. 


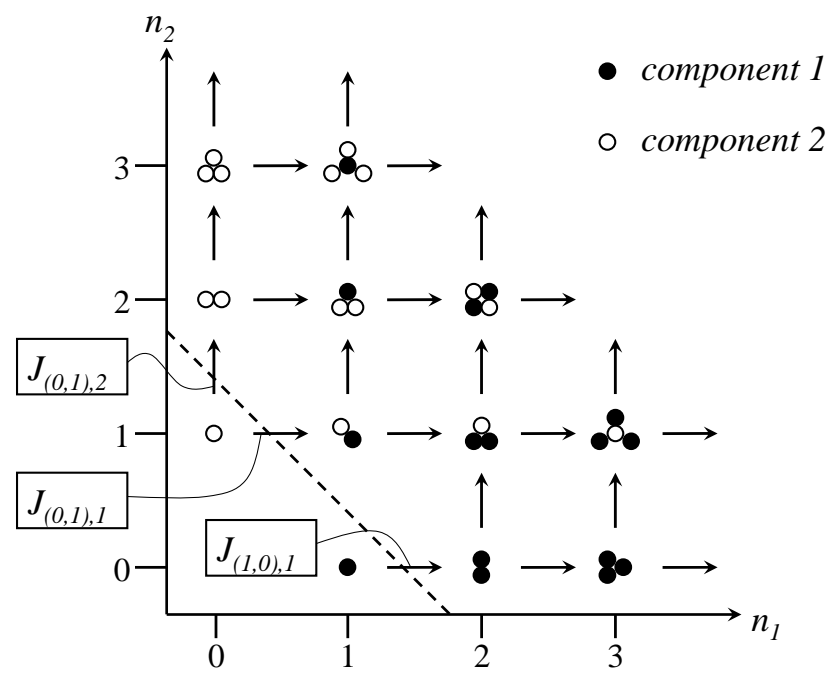

FIGURE 5.3: Close-up of the binary computational domain at the lower boundary (indicated by the dashed line); the arrows represent the fluxes between the various clusters, and the boundary fluxes $J_{(1,0), 1}, J_{(0,1), 1}$, and $J_{(0,1), 2}$ are indicated.

To solve Eqs. (5.20) and (5.29) for the second test case, a space marching algorithm is used, which also employs Heun's predictor-corrector method to perform the numerical integration. The calculation starts at a position which is located slightly downstream of the throat, and proceeds until the nozzle exit is reached. In contrast with the first test case, it is necessary here to extend the binary computational domain to include droplets with radii of over $\sim 10^{-8} \mathrm{~m}$, in order to adequately capture the effects of vapor depletion and latent heat release. Therefore, the BKE is solved on the domain $0 \leq n_{1} \leq 10^{6}, 0 \leq n_{2} \leq 10^{6}$, with $\|\mathbf{n}\|>1$, which allows the binary DSD to be captured in almost full extent. To reduce the computational effort, a sectional approximation is used, which is very similar to the one previously presented in chapter 3 for the single component KE. As illustrated in Figure 5.4, a set of neighboring droplets is grouped into a bin, and each bin exchanges fluxes with its neighbors. The $(k, l)$-th bin is defined as:

$$
\Omega_{k, l}=\left\{\left(n_{1}, n_{2}\right)^{T} \in \mathbb{N}^{2} \mid n_{(k, l)}^{L} \leq n_{1} \leq n_{(k, l)}^{R}, n_{(k, l)}^{B} \leq n_{2} \leq n_{(k, l)}^{T}\right\},
$$

where $n_{(k, l)}^{L}, n_{(k, l)}^{R}, n_{(k, l)}^{B}$, and $n_{(k, l)}^{T}$ denote the limiting values of $n_{1}$ and $n_{2}$ at the left (L), right (R), bottom (B), and top (T) boundaries of $\Omega_{k, l}$, respectively. The left, right, bottom and top boundaries are defined by:

$$
\partial \Omega_{k, l}^{L}=\left\{\left(n_{1}, n_{2}\right)^{T} \in \Omega_{k, l} \mid n_{1}=n_{(k, l)}^{L}\right\},
$$




$$
\begin{aligned}
& \partial \Omega_{k, l}^{R}=\left\{\left(n_{1}, n_{2}\right)^{T} \in \Omega_{k, l} \mid n_{1}=n_{(k, l)}^{R}\right\}, \\
& \partial \Omega_{k, l}^{B}=\left\{\left(n_{1}, n_{2}\right)^{T} \in \Omega_{k, l} \mid n_{2}=n_{(k, l)}^{B}\right\}, \\
& \partial \Omega_{k, l}^{T}=\left\{\left(n_{1}, n_{2}\right)^{T} \in \Omega_{k, l} \mid n_{2}=n_{(k, l)}^{T}\right\},
\end{aligned}
$$

respectively.

For each bin the following sectional approximation of the BKE can be written:

$$
\frac{D \bar{\rho}_{(k, l)}}{D t}=\frac{1}{w_{(k, l), 2}}\left(\bar{J}_{(k-1, l), 1}-\bar{J}_{(k, l), 1}\right)+\frac{1}{w_{(k, l), 1}}\left(\bar{J}_{(k, l-1), 2}-\bar{J}_{(k, l), 2}\right) .
$$

where, $\bar{\rho}_{(k, l)}$ denotes the bin-averaged number density for bin $(k, l)$,

$$
\bar{\rho}_{(k, l)}=\frac{1}{w_{(k, l), 1} w_{(k, l), 2}} \sum_{\mathbf{n} \in \Omega_{k, l}} \check{\rho}_{\mathbf{n}},
$$

with:

$$
w_{(k, l), 1}=n_{(k, l)}^{R}-n_{(k, l)}^{L}+1, w_{(k, l), 2}=n_{(k, l)}^{T}-n_{(k, l)}^{B}+1,
$$

and with:

$$
\bar{J}_{(k, l), 1}=\sum_{\mathbf{n} \in \partial \Omega_{k, l}^{R}} J_{\mathbf{n}, 1}
$$

and similarly:

$$
\bar{J}_{2(k, l)}=\sum_{\mathbf{n} \in \partial \Omega_{k, l}^{T}} J_{\mathbf{n}, 2} .
$$

To compute the fluxes $J_{\mathbf{n}, 1}$ and $J_{\mathbf{n}, 2}$ on the boundaries of $\Omega_{k, l}$, the local number density is interpolated from the bin-averaged number densities of neighboring bins.

The reconstruction of the number densities at the bin-edges has to be carried out at every time step and for every bin within the computational domain. For bins that comprise a large number of different droplet sizes (i.e., when $w_{1(k, l)} \gg 1$ and/or $w_{2(k, l)} \gg 1$ ), the summation in Eqs. (5.38) and (5.39) constitutes a computationally expensive task. To reduce the amount of work, the large sum can be approximated by a smaller sum as follows. Suppose the sum $\sum_{n=0}^{N} f(n)$ has to be calculated for some given function $f(n)$. When $f(n)$ is approximated by a polynomial $P_{M}(n)$ (where $M$ denotes the degree of the polynomial), then the sum is decomposed into sums over powers of $n$, e.g., $\sum_{n=0}^{N} n^{k}$, which can be evaluated using Bernoulli numbers $B_{l}$ :

$$
\sum_{n=0}^{N} n^{k}=\frac{1}{k+1} \sum_{l=0}^{k} B_{l}\left(\begin{array}{c}
k+1 \\
l
\end{array}\right) n^{k-l+1}
$$




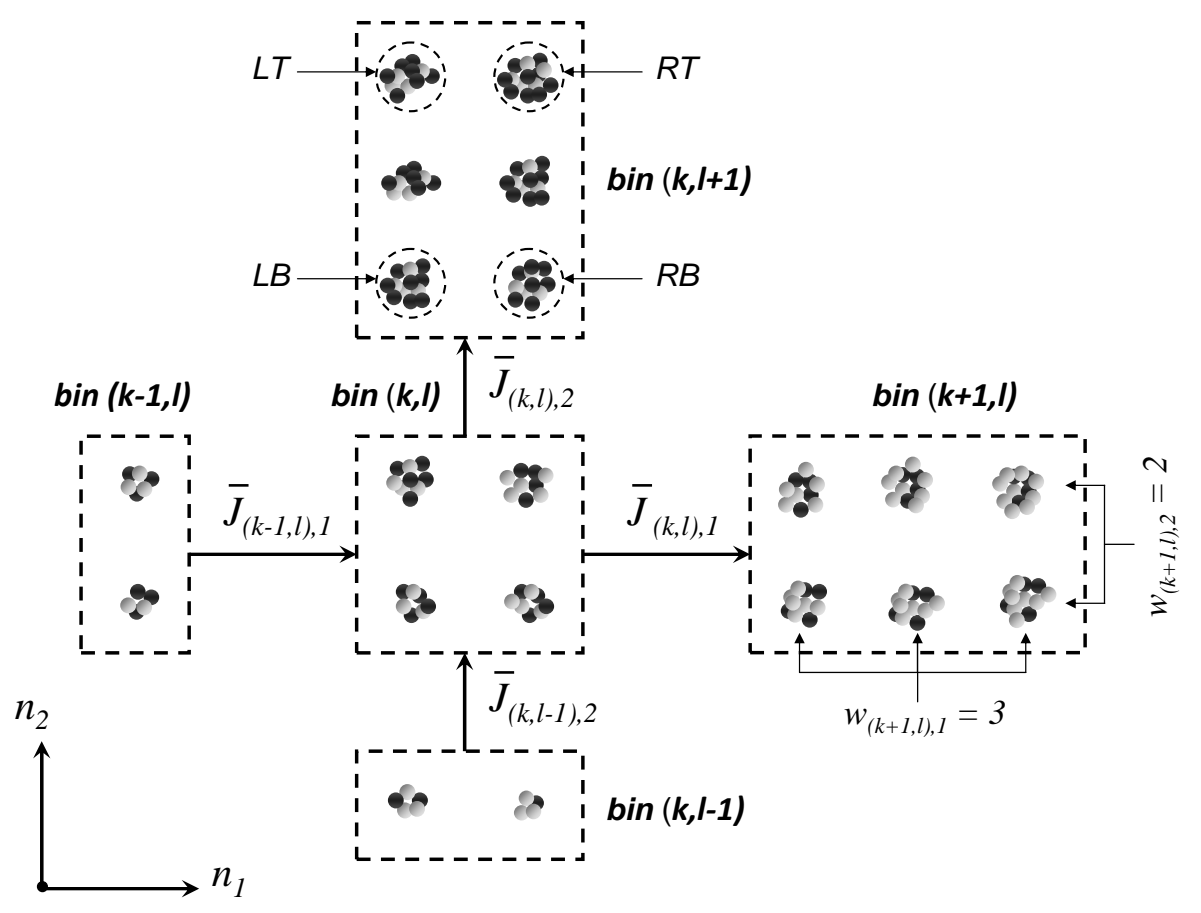

FIGURE 5.4: Schematic representation of the sectional approximation of the BKE in binary space.

The Bernoulli numbers are given by [93]:

$$
B_{0}=1, B_{2 l+1}=0, B_{2 l}=2(-1)^{l+1} \frac{(2 l) !}{\pi^{2 l}\left(2^{2 l}-1\right)} \sum_{j=0}^{\infty}(2 j+1)^{-2 l}, l \geq 1 .
$$

Using Eq. (5.40), the original sum comprising of $N+1$ terms is replaced by a sum comprising of $\frac{1}{2}(M+1)(M+2)$ terms. Hence, if $M^{2}$ is sufficiently small compared to $N$ this implies a significant reduction of work. The Bernoulli numbers can be pre-computed and conveniently stored in computer memory for later use during the simulation.

Once the bin-averaged number densities have been updated, the liquid mass fractions are calculated:

$$
g_{l, m}=m_{v, m} \sum_{\mathbf{n} \in \Omega_{\mathbf{n}}} \check{\rho}_{\mathbf{n}} n_{m} .
$$

Using the sectional approach, this can be written as

$$
g_{l, m}=m_{v, m} \sum_{k} \sum_{l} \sum_{\mathbf{n} \in \Omega_{k, l}} \check{\rho}_{\mathbf{n}} n_{m}
$$


and upon assuming that $\check{\rho}_{\mathbf{n}}$ is sufficiently smooth and small for larger droplets, the second summation can be approximated by:

$$
\sum_{\mathbf{n} \in \Omega_{k, l}} \check{\rho}_{\mathbf{n}} n_{m} \approx \bar{\rho}_{(k, l)} \sum_{\mathbf{n} \in \Omega_{k, l}} n_{m}
$$

The remaining sum can be evaluated for $m=1$ and $m=2$ as:

$$
\begin{aligned}
& \sum_{\mathbf{n} \in \Omega_{k, l}} n_{1}=\frac{1}{2} w_{(k, l), 2}\left\{n_{(k, l)}^{R}\left(n_{(k, l)}^{R}+1\right)-n_{(k, l)}^{L}\left(n_{(k, l)}^{L}-1\right)\right\}, \\
& \sum_{\mathbf{n} \in \Omega_{k, l}} n_{2}=\frac{1}{2} w_{(k, l), 1}\left\{n_{(k, l)}^{T}\left(n_{(k, l)}^{T}+1\right)-n_{(k, l)}^{B}\left(n_{(k, l)}^{B}-1\right)\right\} .
\end{aligned}
$$

In each of the simulations carried out in the first test case, convergence of the numerical integration is verified by performing the solution using successively smaller integration time steps $\Delta t$. Typically, a value of $\Delta t=10^{-10} s$ was found to yield sufficiently converged results. For the simulations in the second test case, a spatial resolution of $\Delta x=10^{-8} \mathrm{~m}$ on a total nozzle length of $0.08 \mathrm{~m}$ was found to yield sufficiently converged solutions for both the binary DSD and the flow variables. With respect to the sectional approximation of the BKE, it was found that a number of $N_{1} \times N_{2}=400 \times 400$ bins yielded sufficiently converged values for the liquid mass fractions. By using a parallel implementation of the current numerical method, the solution for the binary DSD and flow variables is obtained in typical run times of 12 hours.

\subsection{Results and discussion}

\subsubsection{Test case 1: verification of quasisteady-state and transient solu- tions}

\section{Quasisteady-state solution of the BKE}

Before turning to the actual verification of the solutions generated by the numerical method, it is instructive to examine certain salient features of the quasisteady-state solution for the binary DSD. The results obtained for test cases 1.1, 1.2, and 1.3, obtained by using the WW2-equilibrium distribution for calculation of the backward rates, are depicted in Figures 5.5.a, c and e, respectively, whereas the corresponding equilibrium distributions $\hat{\rho}_{\mathbf{n}}^{e q, W W 2}$ are depicted in Figures 5.5.b, d, and f, respectively. The equilibrium distributions show the typical saddle-shaped form, with the position of the saddle point approximately coinciding with the critical composition $\mathbf{n}^{*}$. The departure from equilibrium is shown in Figures 5.6.a, b, c, where the ratio $\hat{\rho}_{\mathbf{n}} / \hat{\rho}_{\mathbf{n}}^{e q, W W 2}$ 
is plotted. Clearly, the quasisteady-state number densities associated with the small clusters are very close to the equilibrium distribution, whereas the larger ones deviate away from it.

For actual verification of the predicted solution, the flux-vectors $\left(J_{\mathbf{n}, 1}, J_{\mathbf{n}, 2}\right)^{T}$ have been computed and plotted in Figures 5.7.a, b, and c, along with the iso-lines for the Gibbs free energy $\Delta G_{\mathbf{n}}$. As can be verified by comparison with figures 2.a, b, and c in [142], the agreement between both sets of predictions appears to be very good, albeit that a comparison between actual numerical data instead of graphs would constitute a more credible verification method.

The data shown in Figures 5.7.a, b, and c also reveals the influence of the vapor activities on the quasisteady state condensation fluxes. For each of the reported activity pairs, the highest magnitude of condensation fluxes passes through the critical composition $\mathbf{n}^{*}$, as expected. The major distinguishing feature between Figures 5.7.a, b, and $\mathrm{c}$ is that the orientation of the condensation flux vectors progressively deviates from the path of minimum energy, as the ratio between the ethanol- and hexanol vapor activities is increased. This behavior was extensively analyzed by Stauffer [114], who correctly noted that in addition to the thermodynamic notion that systems tend to follow the path of steepest descent, it is also important to consider the kinetic effects of monomer impingement. Simply stated, this means that the concentration and mobility of monomers plays a major factor in the path traversed during the nucleation process. This is clearly illustrated when Figure 5.7.a is compared with Figure 5.7.c, where the ratio between the ethanol and hexanol monomers is equal to $\rho_{v, 1} / \rho_{v, 2}=3.7$, and $\rho_{v, 1} / \rho_{v, 2}=42.5$, respectively. The relatively higher ethanol monomer concentration in Figure 5.7.c results in a flux of droplets that is almost parallel to the ethanol-axis, which implies that the majority of large droplets will consist almost entirely of ethanol.

\section{Transient solution of the BKE}

The transient solution of the binary DSD for the ethanol-hexanol system of test case 1.2 is depicted in Figures 5.8.a, b, c, and d, where snapshots are taken at the times $t=2.40 \cdot 10^{-8} \mathrm{~s}$, (b) $t=1.29 \cdot 10^{-7} \mathrm{~s}$, (c) $t=6.93 \cdot 10^{-7} \mathrm{~s}$, and (d) $t=2.00 \cdot 10^{-5} \mathrm{~s}$, respectively. These are the same times for which solution data are reported in [143]. Starting from a zero-distribution at $t=0.0 \mathrm{~s}$, the stream of droplets passes through the saddle region surrounding $\mathbf{n}=\mathbf{n}^{*}$, until a quasisteady state is achieved in Figure 5.8.d.

In the quasisteady state $d \hat{\rho}_{\mathbf{n}} / d t=0$, which consequently leads to

$$
\sum_{\mathbf{n} \in \Omega_{\zeta}} \frac{d \hat{\rho}_{\mathbf{n}}}{d t}=0
$$




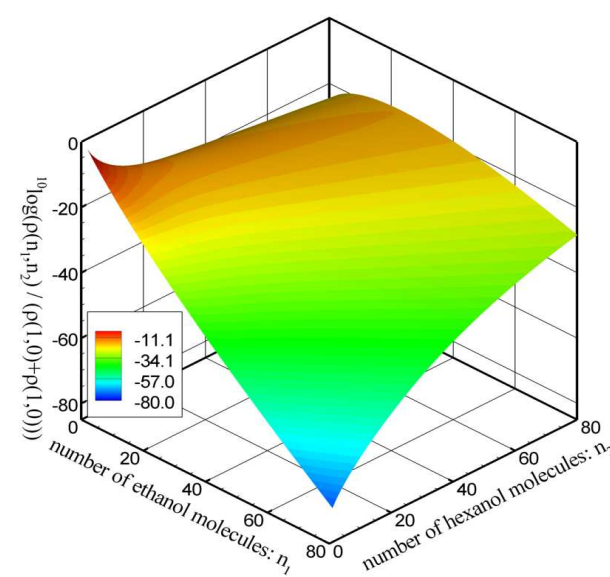

(a)

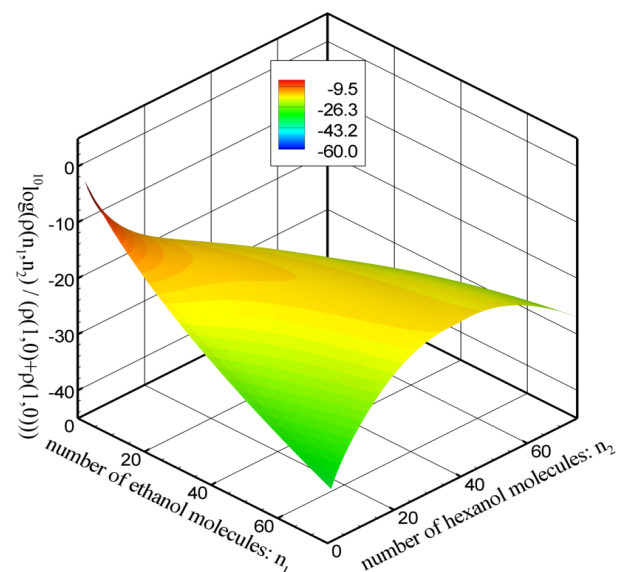

(c)

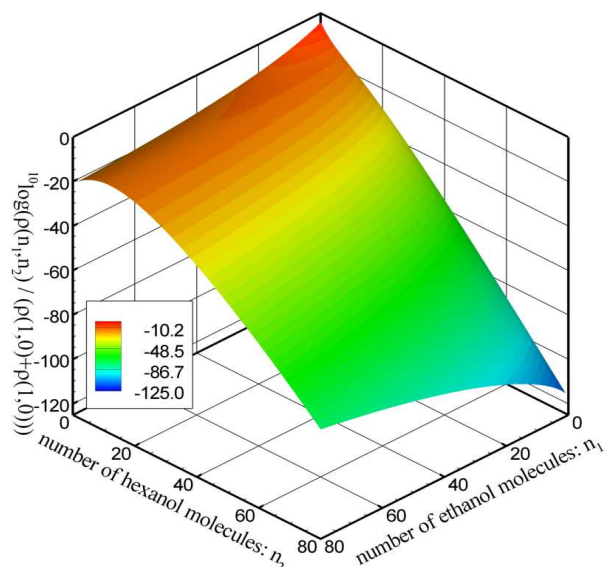

(e)

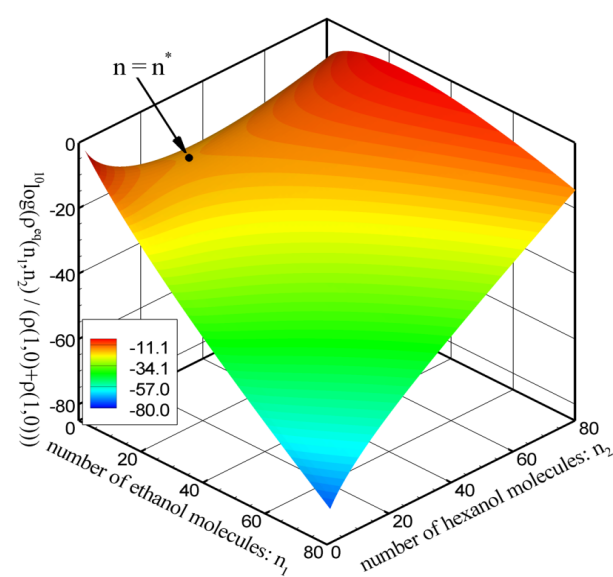

(b)

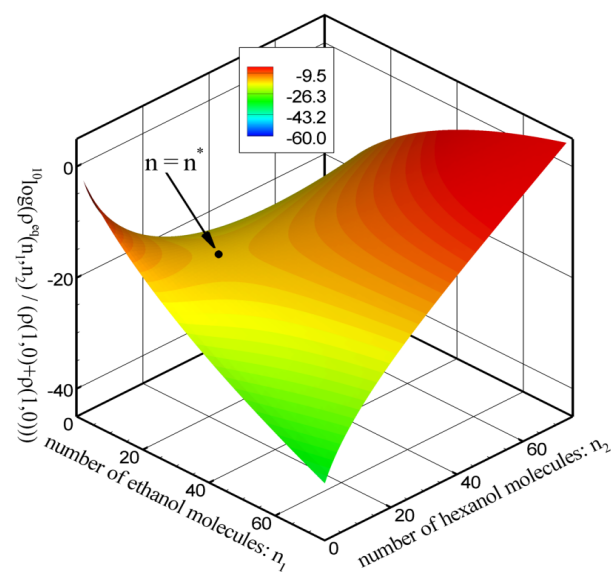

(d)

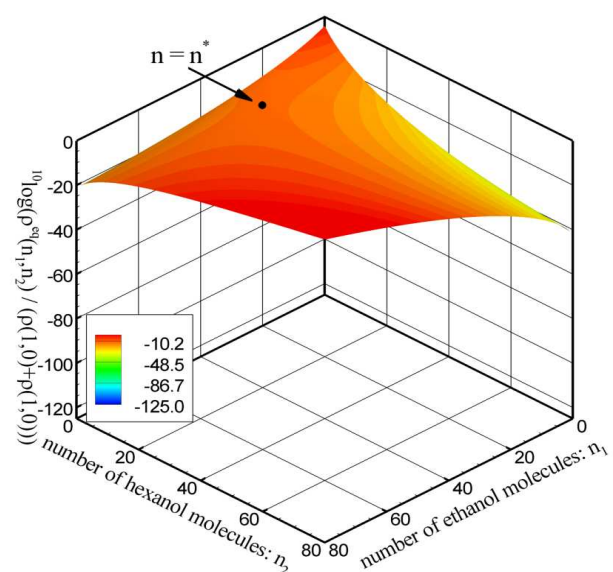

(f)

FIGURE 5.5: Quasisteady-state solutions and corresponding WW2-equilibrium distributions for test cases $1.1(a, b), 1.2(c, d)$, and $1.3(e, f)$. 


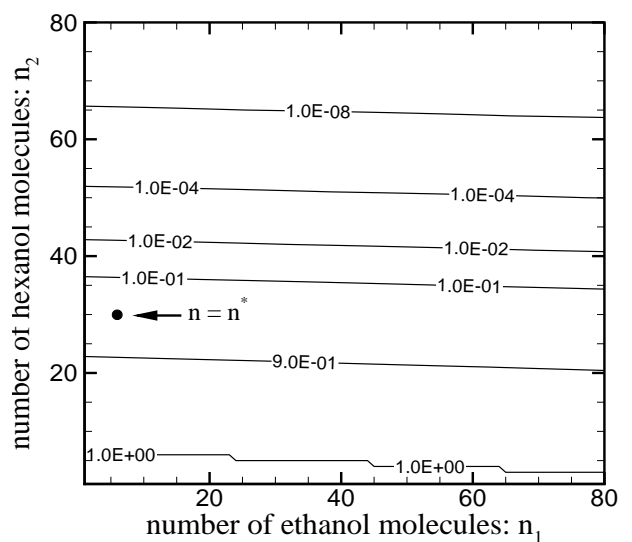

(a)

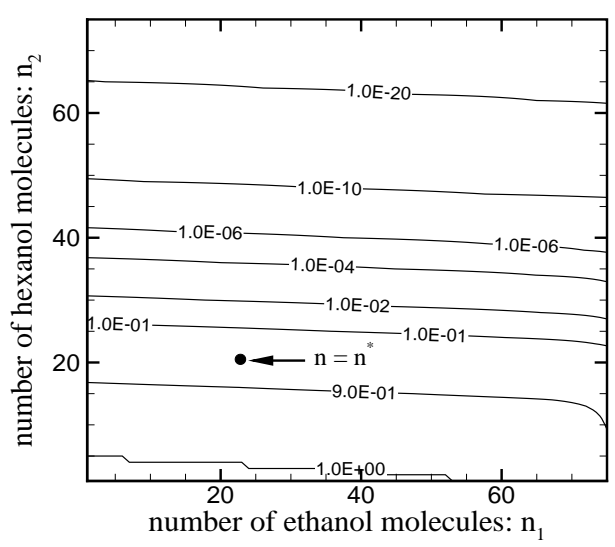

(b)

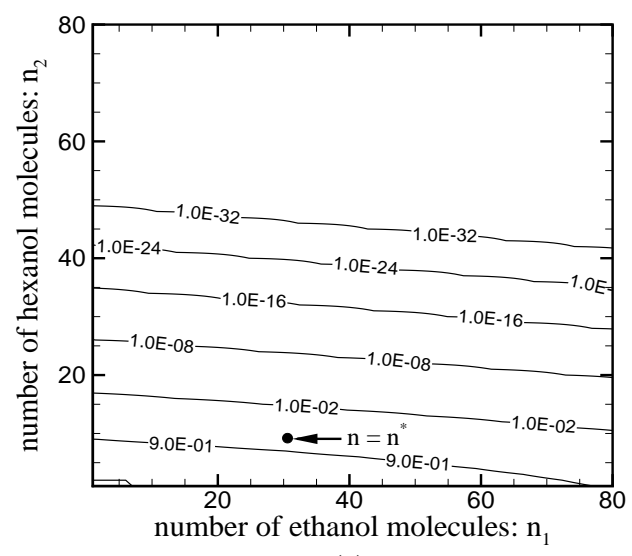

(c)

FIGURE 5.6: Departure from equilibrium, quantified by the ratio $\hat{\rho}_{\mathbf{n}} / \hat{\rho}_{\mathbf{n}}^{e q, W W 2}$, for test cases $1.1(a), 1.2(b)$, and $1.3(c)$.

where $\Omega_{\zeta}$ is the region stretching from the lower boundary (see Figure 5.3) up to and including a curve $\partial \Omega_{\zeta}$ which connects the $n_{2}$-axis with the $n_{1}$-axis. Since

$$
\frac{d \hat{\rho}_{\mathbf{n}}}{d t}=J_{\mathbf{n}_{1}-\mathbf{e}_{1}, 1}-J_{\mathbf{n}, 1}+J_{\mathbf{n}-\mathbf{e}_{2}, 2}-J_{\mathbf{n}, 2},
$$

it follows from Eq. (5.42) that:

$$
\sum_{\mathbf{n} \in \Omega_{\zeta}} J_{\mathbf{n}_{1}-\mathbf{e}_{1}, 1}-J_{\mathbf{n}, 1}+J_{\mathbf{n}-\mathbf{e}_{2}, 2}-J_{\mathbf{n}, 2}=0 .
$$




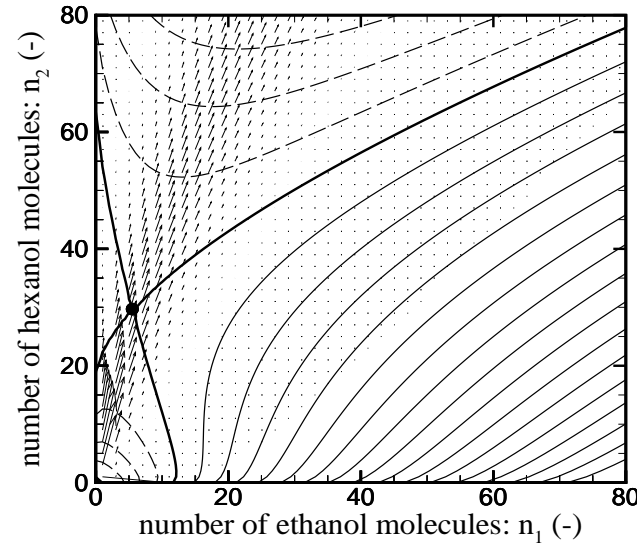

(a)

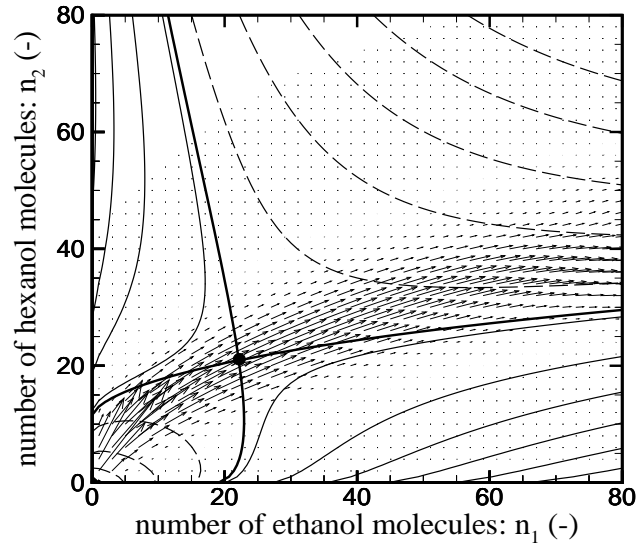

(b)

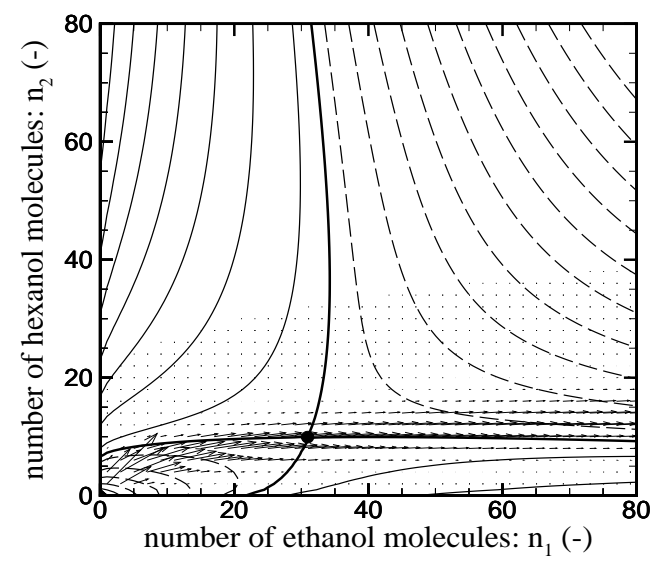

(c)

FIGURE 5.7: Condensation fluxes $\left(J_{\mathbf{n}, 1}, J_{\mathbf{n}, 2}\right)^{T}$ corresponding with the quasisteadystate solution of the BKE for the ethanol-hexanol systems corresponding with the test cases $1.1(a), 1.2(b)$, and $1.3(c)$. The solid circle denotes the saddle point location $\left(\mathbf{n}=\mathbf{n}^{*}\right)$, and the iso-lines correspond with the binary Gibbs free energy $\Delta G_{\mathbf{n}}$ (dashed iso-lines indicate $\Delta G_{\mathbf{n}}$-valleys). These figures correspond with figures 2.a-c of [142].

Due to mutual cancellation of fluxes for neighboring cells, the sum in Eq. (5.43) reduces to a sum of fluxes over the boundaries of $\Omega_{\zeta}$,

$$
\sum_{\mathbf{n} \in \Omega_{\zeta}} J_{\mathbf{n}_{1}-\mathbf{e}_{1}, 1}-J_{\mathbf{n}, 1}+J_{\mathbf{n}-\mathbf{e}_{2}, 2}-J_{\mathbf{n}, 2}=J_{(1,0), 2}+J_{(0,1), 1}+J_{(0,1), 2}-J_{t o t}\left(\partial \Omega_{\zeta}\right),
$$


where:

$$
J_{t o t}\left(\partial \Omega_{\zeta}\right)=\sum_{\mathbf{n} \in \partial \Omega_{\zeta}} J_{\mathbf{n}, 1}+J_{\mathbf{n}, 2}
$$

Combining Eq. (5.43) with Eq. (5.44) thus yields

$$
J_{t o t}\left(\partial \Omega_{\zeta}\right)=J_{(1,0), 2}+J_{(0,1), 1}+J_{(0,1), 2},
$$

which means that $J_{\text {tot }}$ is independent of the curve $\partial \Omega_{\zeta}$ in the quasisteady state.

To verify that the binary DSD shown in Figure 5.8.d indeed corresponds with the quasisteady-state solution, the total flux $J_{t o t}$ is calculated along the line

$$
n_{1}+n_{2}=\zeta,
$$

where the parameter $\zeta$ allows the line to be moved in binary space. Using the numerical solution data obtained for test case 1.2, $J_{t o t}(\zeta)$ has been calculated and plotted in Figure 5.9, for the same times corresponding with Figure 5.8. Here, it is clearly observed that the nucleation rate $J_{t o t}$ evolves in time until it becomes independent of $\zeta$ for $t=2 \cdot 10^{-5} \mathrm{~s}$, which consequently shows that the quasisteady state has indeed been achieved.

For verification of the transient solution, Figures 5.10.a, b, c, and d show the temporal evolution of the sum $J=J_{\mathbf{n}, 1}+J_{\mathbf{n}, 2}$ of condensation fluxes in binary space. As can be verified by comparison with figures 2.a, b and c in [143], the agreement between both numerical predictions is very good. Thus in conclusion, the results obtained for test cases 1.2, 1.2 and 1.3 have demonstrated that the current numerical code is able to accurately predict both the quasisteady-state and transient solution of the BKE.

\subsubsection{Test case 2: nozzle flow with binary condensation}

\section{Validation of flow model with binary condensation}

The solution for the flow field- and thermodynamic variables for test cases 2.1 to 2.5, are depicted in Figures 5.11.a-f. These solutions have been obtained by solving Eq. (5.29) simultaneously with Eq. (5.20), with the backward rates calculated from the WW2-equilibrium distribution. Qualitatively, the depicted trends are similar to the ones observed for single-component condensation (see chapter 3 ). In each case, the influence of condensation becomes noticeable at a certain onset point, where the condensational release of latent heat causes a slight rise in temperature, and a very slight reduction in the Mach number, as shown in Figures 5.11.a and b, respectively. Furthermore, the presence of nucleation zones is observed in the regions where the vapor activities attain extremal values in Figures 5.11.c and d. Downstream of the nucleation zone the vapor activities are significantly smaller, which leads to a reduced rate 


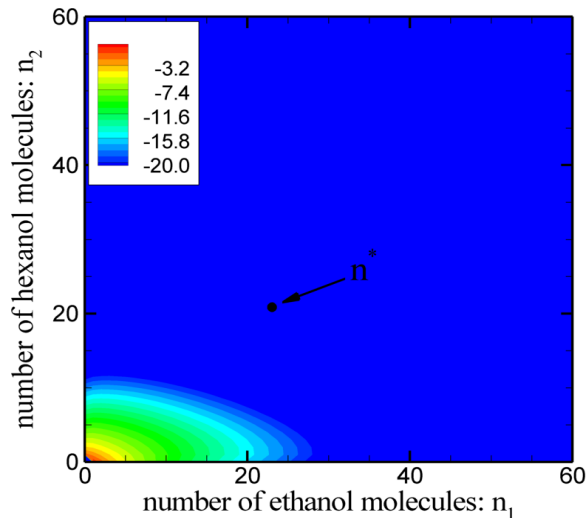

(a)

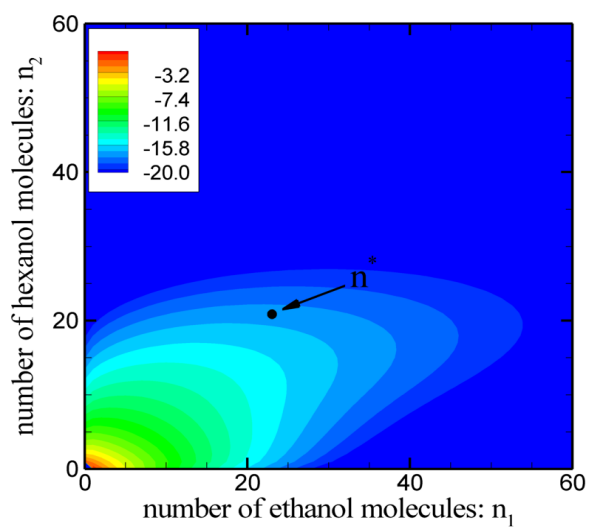

(c)

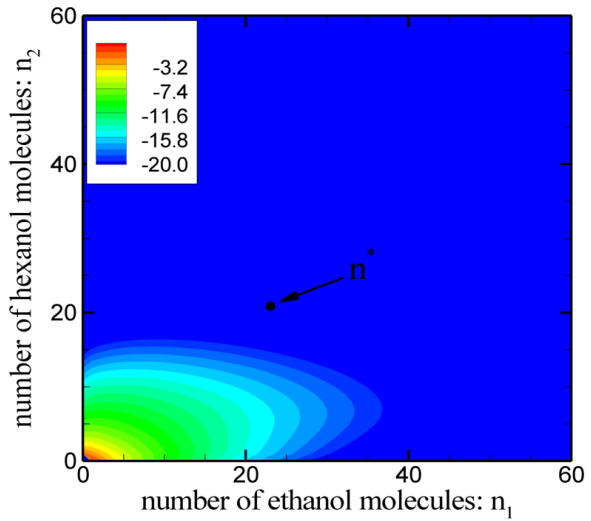

(b)

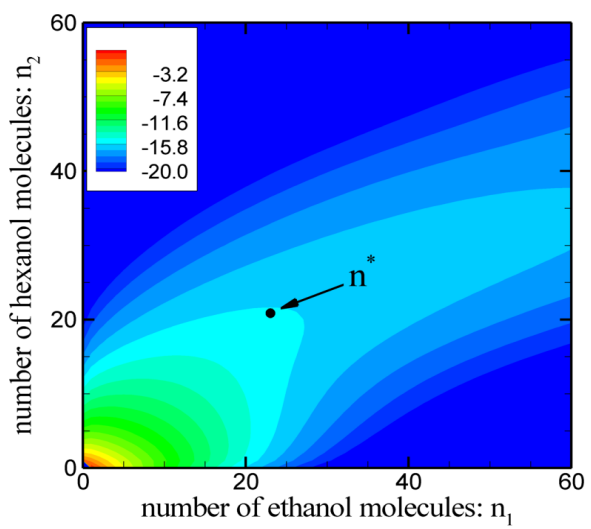

(d)

FIGURE 5.8: Temporal evolution of the binary DSD for an ethanol-hexanol mixture at $T=260.0 \mathrm{~K}$, with respective activities of $a_{v, 1}=1.5$ and $a_{v, 2}=9$ [143]: (a) $t=2.40 \cdot 10^{-8} s$; (b) $t=1.29 \cdot 10^{-7} s$; (c) $t=6.93 \cdot 10^{-7} s ;$ (d) $t=2.00 \cdot 10^{-5} s$ (steady state solution). The contours correspond with ${ }^{10} \log \frac{\hat{\rho}_{\mathbf{n}}}{\hat{\rho}_{(1,0)}+\hat{\rho}_{(0,1)}}$; the solid circle indicates the position of the saddle-point $\left(\mathbf{n}=\mathbf{n}^{*}\right)$.

of liquid production, as can be observed from the component liquid mass fractions depicted in Figures 5.11.e and $\mathrm{f}$.

The solution for the binary DSD at the nozzle exit is shown in Figures 5.12.a, b, and $\mathrm{c}$ for the test cases 2.2, 2.3 and 2.4, respectively. The influence of the initial vapor composition is qualitatively similar to what has been observed for the ethanolhexanol systems studied in test cases 1.1-1.3. As the inlet vapor composition becomes richer in ethanol, the flux of droplets draws increasingly more parallel to the ethanol axis, so that large droplets consist of ethanol almost exclusively. 


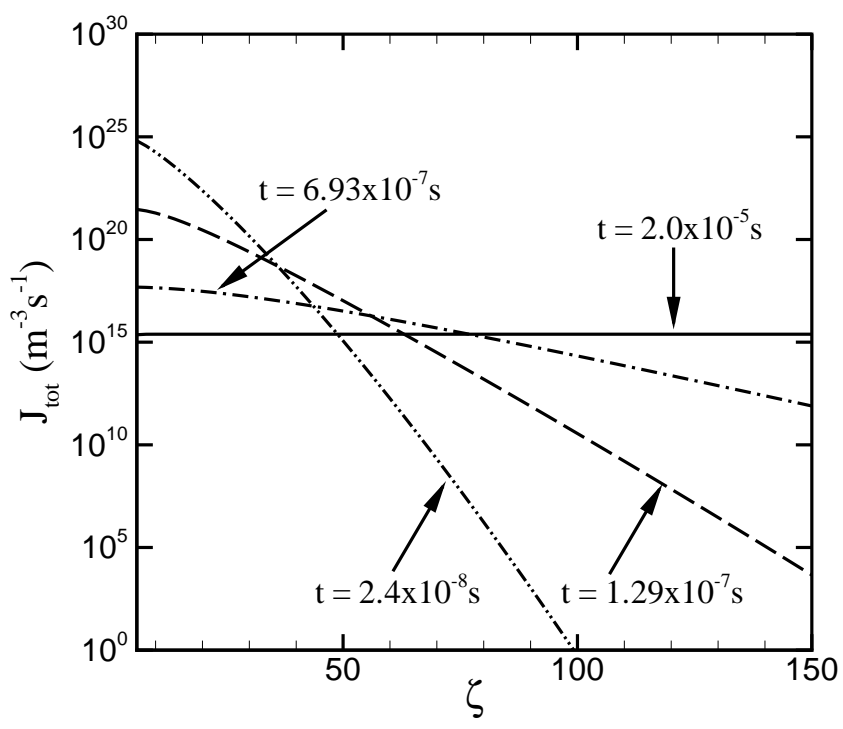

FIGURE 5.9: Temporal evolution of the total flux $J_{\text {tot }}(\zeta)$ measured along the line $n_{1}+$ $n_{2}=\zeta$, for test case 1.2.

To validate the condensation model, the temperatures and partial vapor pressures at condensation-onset are compared with the measurement data reported in [144]. Here, the onset-point of condensation is defined as the position along the nozzle axis where the temperature of the condensing flow deviates $0.5 \mathrm{~K}$ from that of a dry flow under choked conditions. The predicted and measured data are listed in table 5.2, with corresponding plots given in Figures 5.13.a, b and c. The agreement between both data sets is fairly good, given that the flow model is inviscid and only quasi-onedimensional, and that the condensation model is based on macroscopic theory. The latter is especially remarkable, since at condensation onset, the liquid dispersion consists predominantly of droplets that typically contain only a few tens of molecules, so that one would expect the macroscopic theory to be invalid. The predictions obtained with the current model seem to suggest, however, that the WW2-equilibrium distribution, which was derived from pragmatic arguments in [135], may still give a reliable description of the average cluster dynamics at the microscopic level. It is noted, however, that comparison of condensation onset data is not sufficient to validate the current model, as one would rather use a complete pressure, temperature and/or vapor saturation profile measured over the entire nozzle length to make a more thorough analysis. Contrary to unary condensation, reliable data for binary condensation in nozzles is hardly available; to the author's knowledge, the data given by Tanimura et al. in [120] are the only reported measurements on binary condens- 


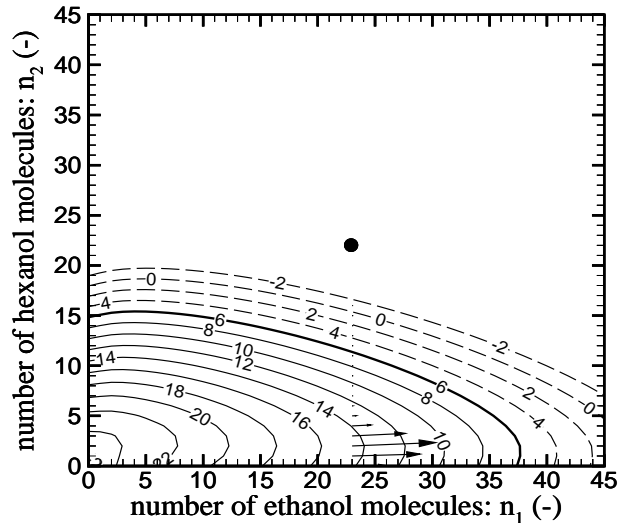

(a)

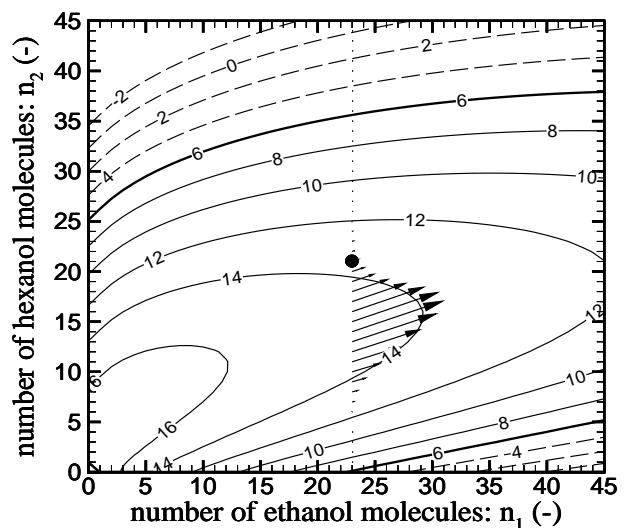

(c)

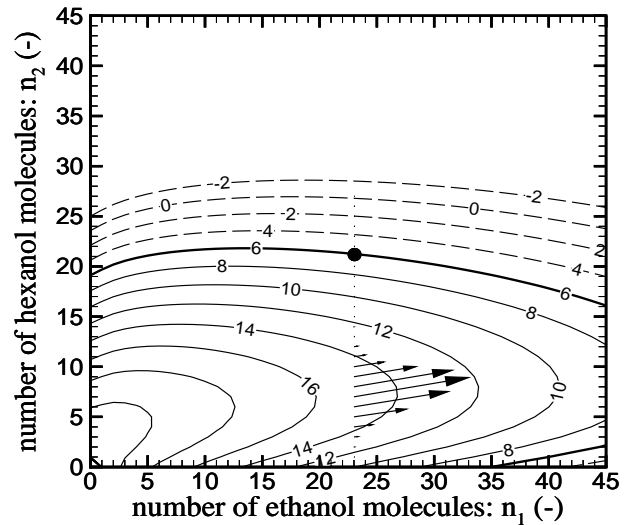

(b)

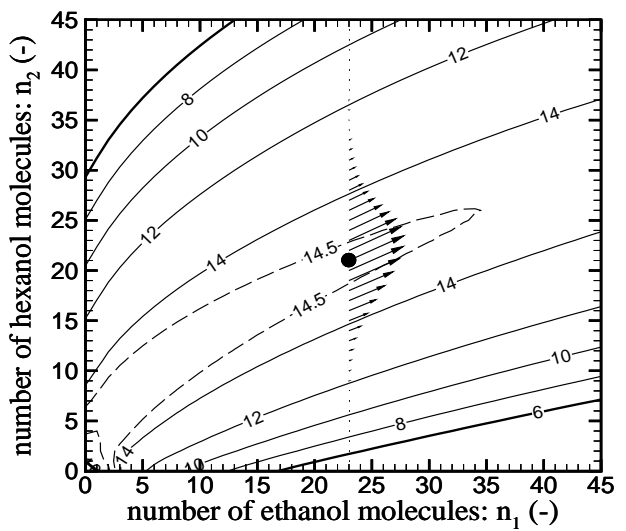

(d)

FIGURE 5.10: Temporal evolution of the sum $J=J_{\mathbf{n}, 1}+J_{\mathbf{n}, 2}$ of binary fluxes for test case 1.2 (ethanol-hexanol mixture at $T=260.0 \mathrm{~K}$, with respective activities of $a_{v, 1}=1.5$ and $a_{v, 2}=9$ ), such as reported in figures $2 . a-d$ of [143]: (a) $t=2.40 \cdot 10^{-8} s ;(b)$ $t=1.29 \cdot 10^{-7} s ;(c) t=6.93 \cdot 10^{-7} s ;(d) t=2.00 \cdot 10^{-5} s$ (steady state solution). The iso-lines correspond with ${ }^{10} \log J$, with $J$ expressed in $m^{-3} s^{-1}$. The solid circle indicates the position of the saddle-point $\left(\mathbf{n}=\mathbf{n}^{*}\right)$, and the arrows represent the condensation flux vectors $\left(J_{\mathbf{n}, 1}, J_{\mathbf{n}, 2}\right)^{T}$ at a vertical line passing through $\mathbf{n}^{*}$.

ing nozzle flow. Because they have used a strongly non-ideal mixture of ethanol and heavy water $\left(\mathrm{D}_{2} \mathrm{O}\right)$ as the condensing vapors in [120], the current model is not suited for application to their experiments. It is anticipated that surface enrichment effects need to be incorporated in the current condensation model, in order to use the measurements reported in [120] for validation purposes. 


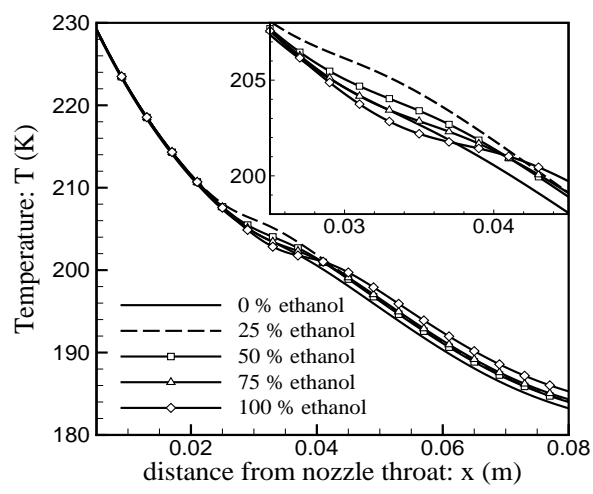

(a)

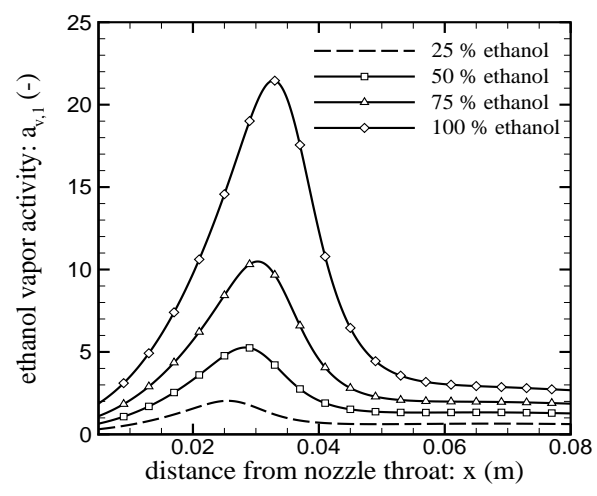

(c)

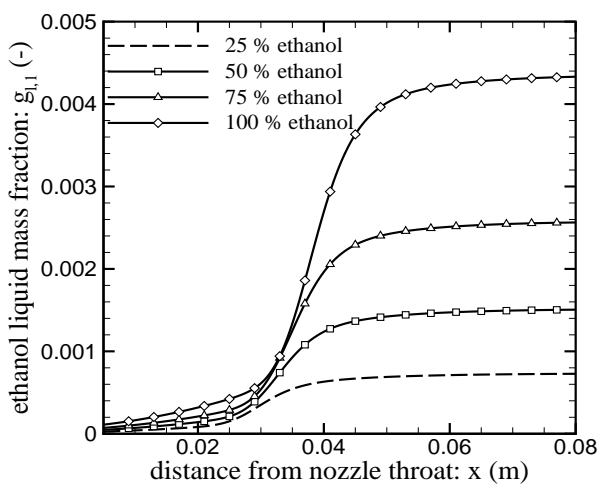

(e)

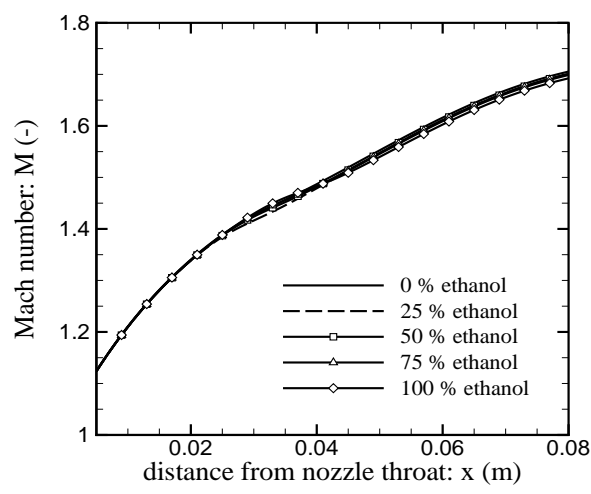

(b)

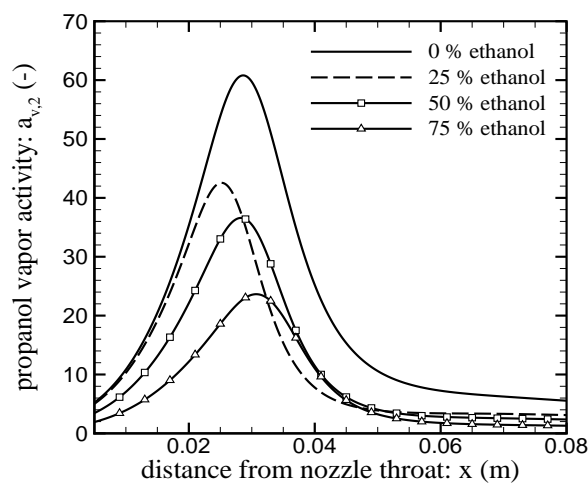

(d)

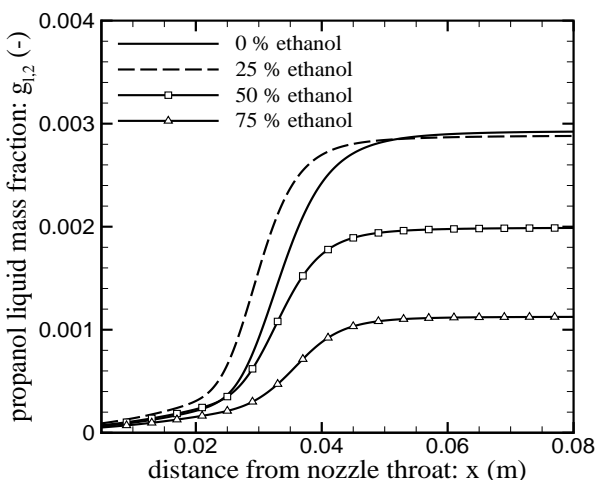

(f)

Figure 5.11: Solution of flow field and thermodynamic variables for test cases 2.1 to 2.5 (ethanol percentages are molar fractions, see table 5.1 for details): (a) temperature (with insert for the nucleation zone), (b) Mach number, (c) ethanol and (d) propanol vapor activities, $(e)$ ethanol and $(f)$ propanol liquid mass fractions. 


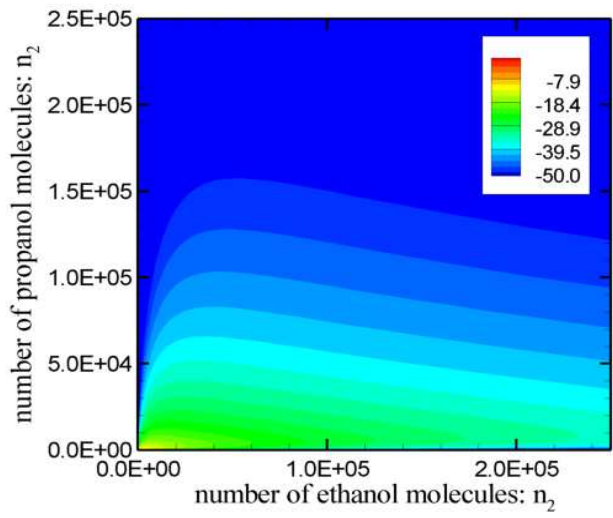

(a)

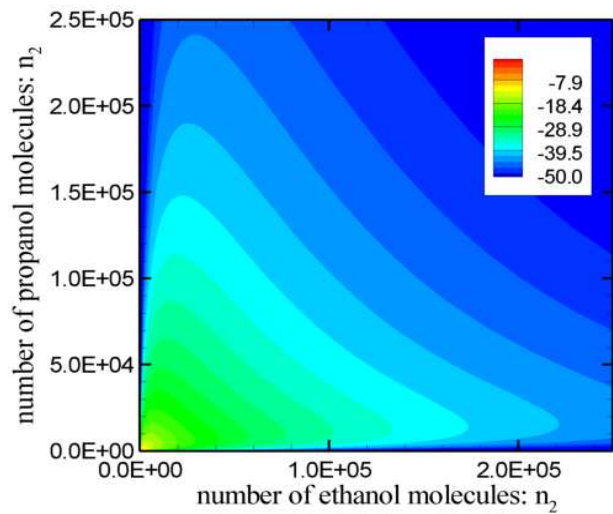

(b)

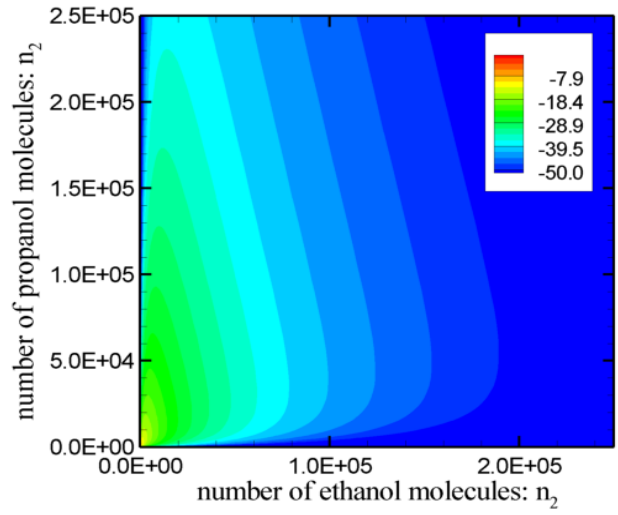

(c)

FIGURE 5.12: Binary DSD at the nozzle exit $(x=0.08 m)$, for test cases $(a) 2.2,(b)$ 2.3, and (c) 2.4. The contours correspond with ${ }^{10} \log \frac{\hat{\rho}_{\mathbf{n}}}{\hat{\rho}_{(1,0)}+\hat{\rho}_{(0,1)}}$. 


\begin{tabular}{ccccccc}
\hline \hline case no.: & $p_{v, 1}^{\text {exp }}(\mathrm{Pa})$ & $p_{v, 1}^{\text {num }}(\mathrm{Pa})$ & $p_{v, 2}^{\text {exp }}(\mathrm{Pa})$ & $p_{v, 2}^{\text {num }}(\mathrm{Pa})$ & $T^{\text {exp }}(\mathrm{K})$ & $T^{\text {num }}(\mathrm{K})$ \\
\hline 2.1 & 52.6 & 49.2 & $\ldots$ & $\ldots$ & 210.2 & 210.9 \\
2.2 & 30.9 & 30.1 & 10.3 & 9.5 & 209.5 & 212.8 \\
2.3 & 18.5 & 17.5 & 18.5 & 17.2 & 210.7 & 212.8 \\
2.4 & 9.3 & 8.4 & 27.8 & 25.4 & 212.9 & 213.2 \\
2.5 & $\ldots$ & $\ldots$ & 27.6 & 22.9 & 211.4 & 207.6 \\
\hline \hline
\end{tabular}

TABLE 5.2: Comparison of experimental (index 'exp', from [144]) and simulation data (index 'num') for vapor pressures $p_{v, k}$ and temperature $T$ at condensation onset. The simulation employs the WW2-equilibrium distribution to compute the backward rates in the BKE; the uncertainty in the measured onset temperature is $\pm 1 K$, and the relative uncertainty in the measured pressure is $5 \%$.

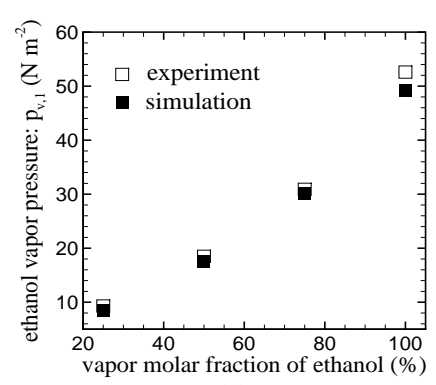

(a)

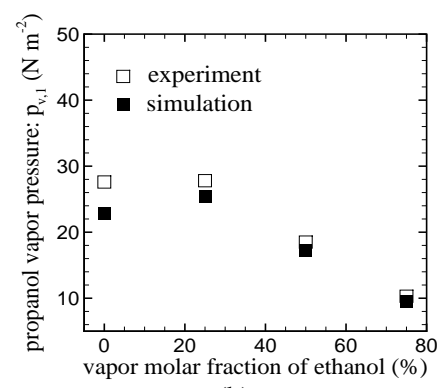

(b)

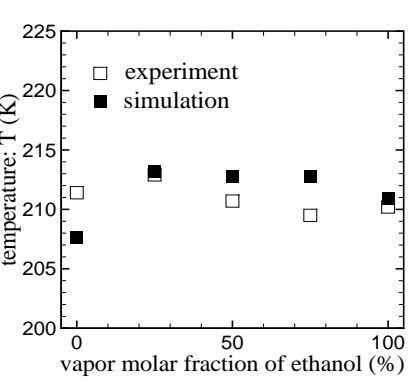

(c)

FIGURE 5.13: Comparison of condensation-onset data for test case 2 (the figures correspond with the data of table 5.2): onset vapor pressures for ethanol (a) and propanol (b); (c) onset temperatures .

\section{Analysis of the spatial variation of the binary DSD}

In this section it is examined whether or not condensing nozzle flows are suitable for extracting data on quasisteady-state nucleation. For nozzle flows with unary condensation, it has been demonstrated in chapter 3 that rapid expansion can result in significant deviations from results predicted with quasisteady-state nucleation theory. In this section, the changes in the binary DSD along a Lagrangian trajectory are analyzed. Along such a path, the Lagrangian variation in $\check{\rho}_{\mathbf{n}}$ can be compared to the transient behavior of $\hat{\rho}_{\mathbf{n}}$ observed in the stagnant systems of test cases $1.1-1.3$.

Using the simulation of test case 2.3 (50\% ethanol), the contours of the dimensionless number density $\left.\check{\rho}_{\mathbf{n}} / \check{\rho}_{(1,0)}+\check{\rho}_{(0,1)}\right)$ have been plotted in Figures 5.14.a, b, c and d for the positions $x=0.019 m, x=0.029 m, x=0.036 m$, and $x=0.060 m$, respectively. To actually verify whether or not the solution is locally in a quasisteady state, the total flux $J_{t o t}(\zeta)$ is again calculated, similar to what was done in the transient analysis 
for test case 1.2. The difference here, however, is that a different line is chosen along which $J_{t o t}$ is measured. Due to the sectional approach of solving the BKE, it is more convenient to measure $J_{\text {tot }}$ along the lines $n_{1}=\zeta$, and $n_{2}=\zeta$, from the point at which they intersect the coordinate axes, up to their mutual intersection point (see Figure 5.15). The results for $J_{t o t}(\zeta)$, calculated at the five positions $x=0.019 m, x=0.025 m$, $x=0.029 m, x=0.036 m$, and $x=0.060 m$, are shown in Figure 5.16.a, with a separate plot for $x=0.060 \mathrm{~m}$ in Figure 5.16.b. The first four positions lie within the nucleation zone, as can be seen from the vapor activities shown in Figures 5.11.c and d. Considering the curves for $J_{t o t}$ for these positions, it is clear that the binary DSD does not achieve quasisteady-state behavior within the nucleation zone. Near the end of this region $(x=0.036 \mathrm{~m})$, it is even observed that the total flux is negative for small droplets, whereas large droplets $(\zeta>11)$ show a positive total flux. For the position $x=0.060 \mathrm{~m}$ located far downstream of the nucleation zone, the total flux is much smaller compared to its value in the nucleation zone. Figure 5.16.b shows that $J_{t o t}$ is negative for small clusters, whereas $J_{t o t}>0$ for large clusters $(\zeta>100)$. This relatively small positive flux is also noticeable as the low rate of liquid production in Figures 5.11.e and $\mathrm{f}$.

These results clearly illustrate that quasisteady-state nucleation is not always achieved during supersonic expansions. The conclusion drawn from this is that caution should be exercised when analyzing the measurement data obtained from condensing nozzle flow experiments based on quasisteady-state nucleation theory.

\section{Influence of equilibrium distribution}

In this section, the influence of the binary equilibrium distribution on the flow field variables is investigated. The simulations are carried out for test cases 2.2, 2.3 and 2.4, with either the WW1- or WW2-equilibrium distribution used for calculating the backward rates in the BKE. The results are shown in Figures 5.17.a - f, where the temperature and component liquid mass fractions have been plotted for each of the cases mentioned. Clearly, both the temperature and liquid mass fractions based on $\hat{\rho}_{\mathbf{n}}^{e q, W W 1}$ are very much different from the ones corresponding with $\hat{\rho}_{\mathbf{n}}^{e q, W W 2}$. The use of $\hat{\rho}_{\mathbf{n}}^{e q, W W 1}$ consistently delays the onset of condensation until halfway the divergent section of the nozzle, where the liquid mass fractions start to rise. This difference between the results predicted with $\hat{\rho}_{\mathbf{n}}^{e q, W W 1}$ and $\hat{\rho}_{\mathbf{n}}^{e q, W W 2}$ is actually comparable to what has been observed in the unary case, when the results predicted with the Courtney equilibrium distribution, are compared to those of the SCC-equilibrium distribution (see chapter 3). In essence, the fully consistent character of the WW2-equilibrium distribution causes $\hat{\rho}_{\mathbf{n}}^{e q, W W 2}>\hat{\rho}_{\mathbf{n}}^{e q, W W 2}$ for small clusters. As the number densities associated with the small clusters are very near the equilibrium value, and because the liquid dispersion is dominated by these small droplets at condensation-onset, it is

concluded that using $\hat{\rho}_{\mathbf{n}}^{e q, W W 1}$ to calculate the backward rate should indeed delay the 


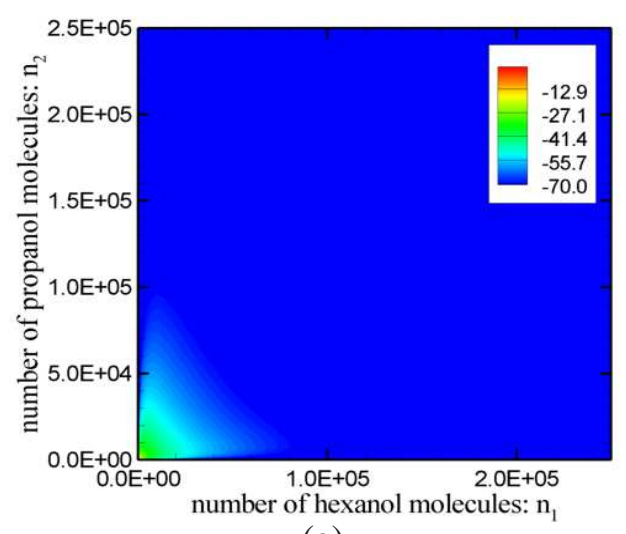

(a)

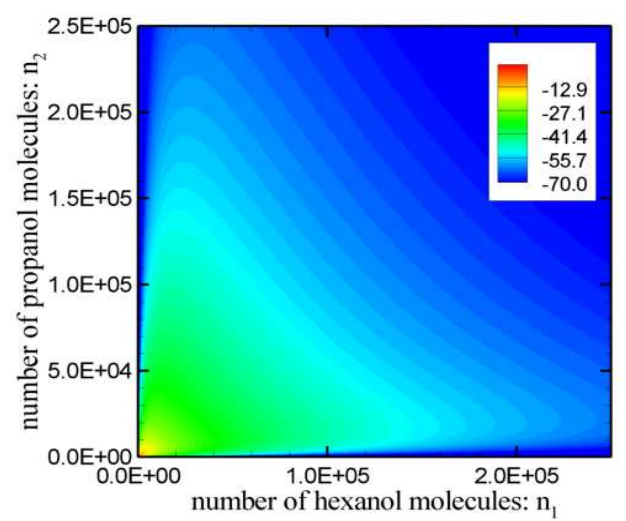

(c)

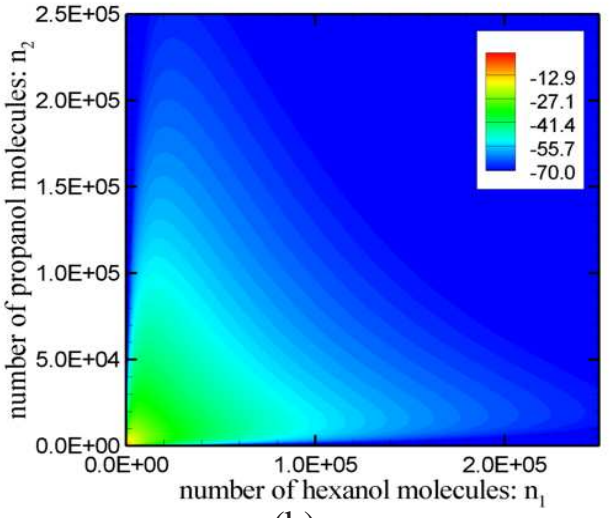

(b)

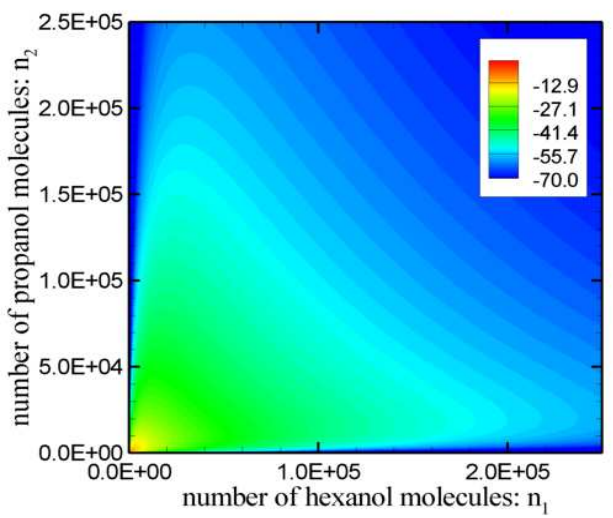

(d)

FIGURE 5.14: Spatial evolution of the binary DSD corresponding with test case 2.3 (50\% ethanol): (a) $x=0.019 m$, (b) $x=0.029 m$, (c) $x=0.036 m$, and (d) $x=0.060 m$. The contours correspond with ${ }^{10} \log \frac{\check{\rho}_{\mathbf{n}}}{\check{\rho}_{(1,0)}+\check{\rho}_{(0,1)}}$.

onset of condensation.

Given the reasonable agreement found between the onset temperatures and vapor pressures from experiment and simulation with the WW2-equilibrium distribution (see table 5.2), it is thus concluded that the WW1-equilibrium distribution yields a rather poor description of the average cluster dynamics at the microscopic scale. This is apparently in contrast with the case of unary condensation, for which the Courtneyequilibrium distribution was found to perform much better than the fully consistent SCC-equilibrium distribution. 


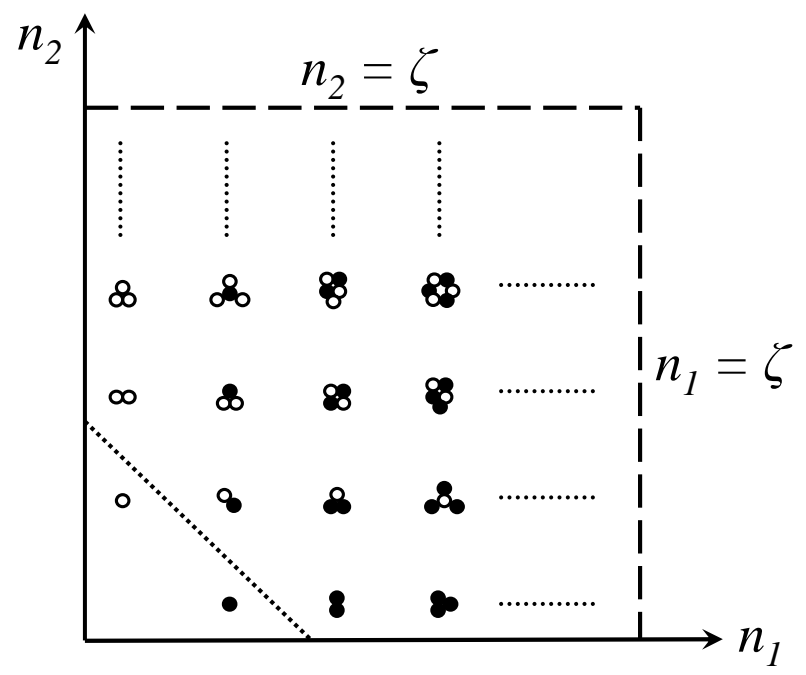

FIGURE 5.15: Measurement line (dashed) for the total flux $J_{\text {tot }}(\zeta)$ in test case 2. The dotted line denotes the lower size boundary.

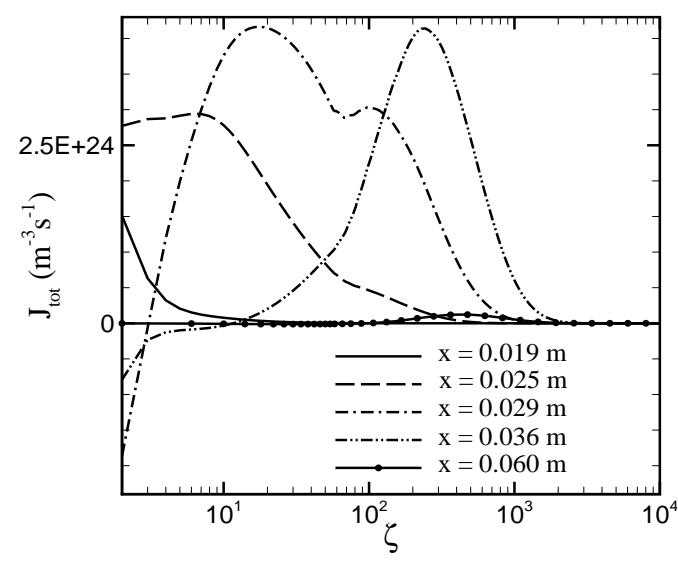

(a)

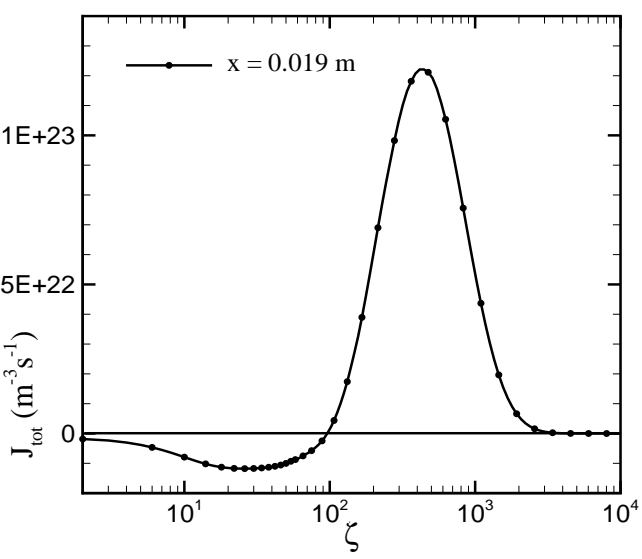

(b)

FIGURE 5.16: Total flux $J_{\text {tot }}(\zeta)$ plotted at various positions along the nozzle axis $(a)$, with a separate plot for $J_{\text {tot }}$ at $x=0.060 \mathrm{~m}(b)$. 


\subsection{Conclusions}

The kinetic equation for the binary droplet size distribution (DSD) has been solved numerically for a simulated nucleation experiment and condensing flow in a nozzle.

The first test case has been used to verify that the numerical method is able to accurately solve the binary kinetic equation (BKE), whereas the second one is used for validation of the condensing flow model by means of comparison with actual measurement data from [144].

The verification has proven successful for each simulated nucleation experiment. Furthermore, the influence of the vapor composition on the resulting binary DSD has been examined, which is found to be in agreement with the analysis by Stauffer [114].

The validation of the condensation model has been carried out for five test cases, with different inlet compositions of the vapor phase. The predicted temperatures and pressures at condensation-onset are found to be in good agreement with measurement data, when the fully self-consistent WW2-equilibrium distribution is used to calculate the backward rates in the BKE.

By analyzing the total condensation flux in binary space, it has been found that the binary DSD does not approach a Lagrangian quasisteady-state solution during the rapid expansions considered here. The conclusion drawn from this is that caution should be exercised when analyzing the measurement data obtained from condensing nozzle flow experiments based on quasisteady-state nucleation theory.

Finally, the sensitivity of the flow field solution to the equilibrium distribution has been quantified. The results obtained by calculating the backward rates in the BKE using either the partially self-consistent WW1-equilibrium distribution, or the fully self-consistent WW2-equilibrium distribution, show profound differences in terms of predicted temperatures and component liquid mass fractions. Furthermore, the condensation-onset data predicted by using the WW1-equilibrium distribution are shown to be much different from the reported measurements. 


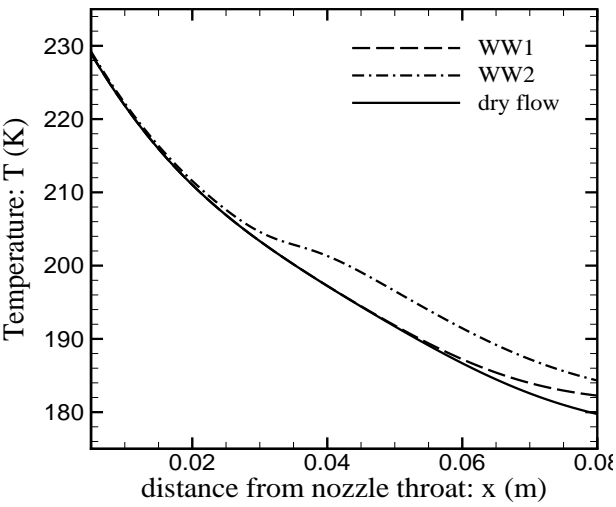

(a)

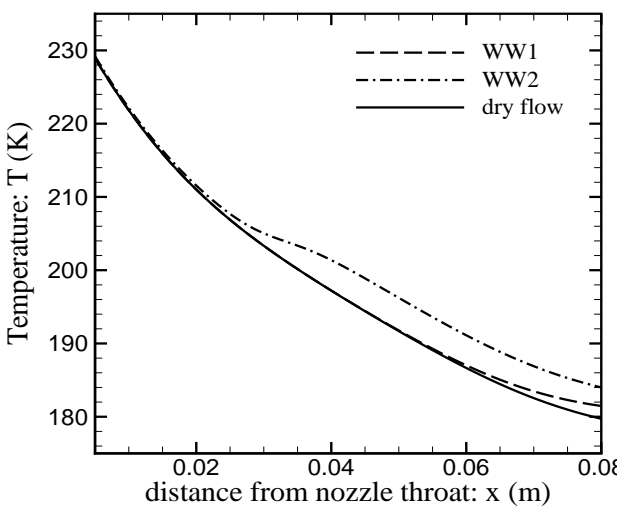

(c)

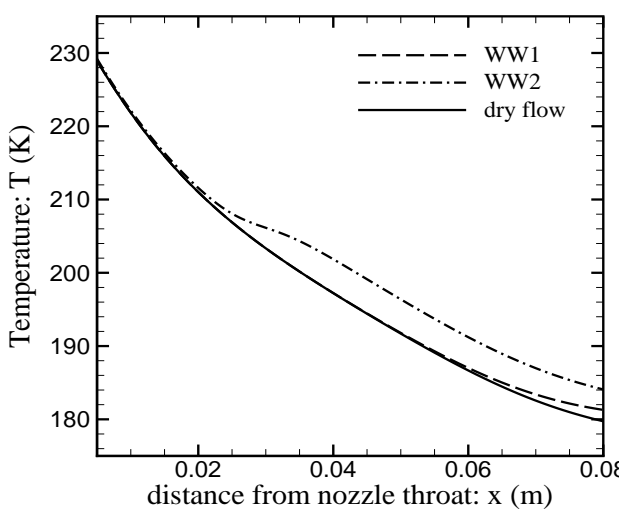

(e)

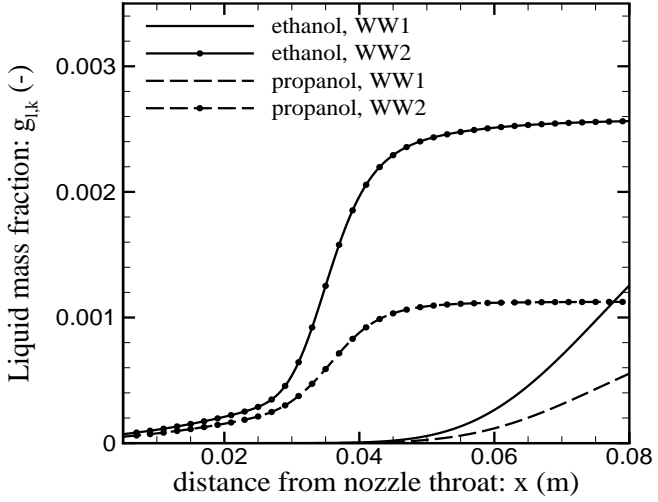

(b)

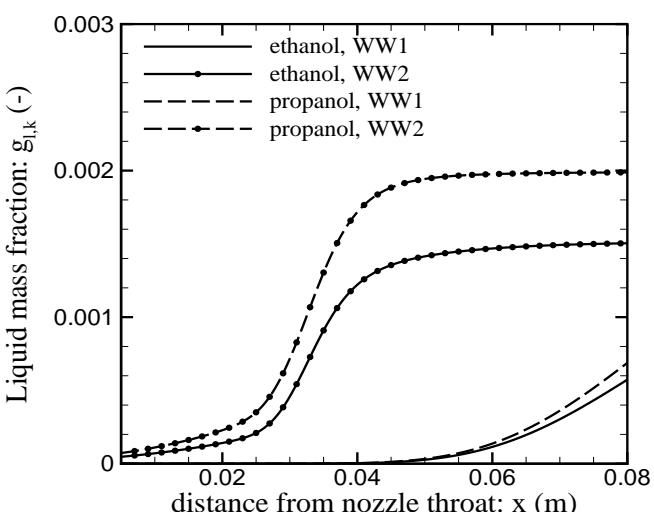

(d)

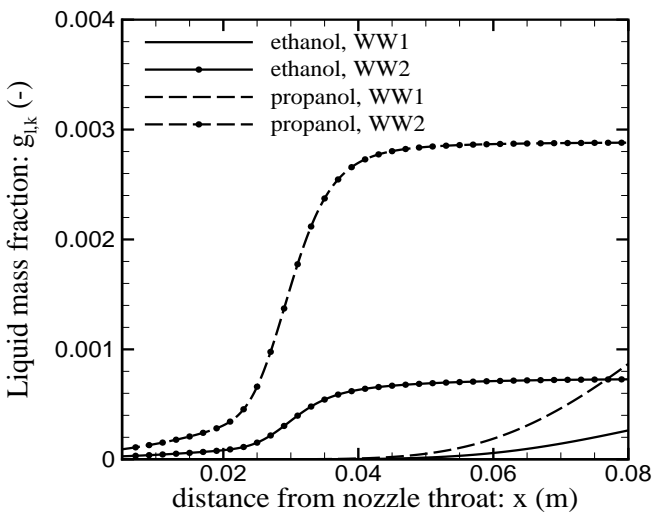

(f)

FIGURE 5.17: Comparison of temperature and liquid mass fraction profiles for simulations in which the backward rates in the BKE are computed by using either $\hat{\rho}_{\mathbf{n}}^{e q, W W 1}$ or $\hat{\rho}_{\mathbf{n}}^{e q, W W 2}$ for test cases $2.2(a, b), 2.3(c, d)$, and $2.4(e, f)$. 


\section{DROPLET CONDENSATION IN SYNTHETIC TURBULENCE}

The condensation of micro-droplets in a synthetic turbulent flow, reminiscent of atmospheric clouds, is investigated numerically and analytically. Droplets have been followed through a synthetic turbulent flow field composed of 200 random Fourier modes, with wave numbers ranging from the integral scales $\left(O\left(10^{2} \mathrm{~m}\right)\right)$ to the Kolmogorov scales $\left(O\left(10^{-3} \mathrm{~m}\right)\right.$ ). As the influence of all turbulence scales is investigated, direct numerical simulation is not practicable, making kinematic simulation the only viable alternative. Two fully Lagrangian droplet growth models are proposed: a one-way coupled model in which only adiabatic cooling of a rising air parcel is considered, and a two-way coupled model which also accounts for the effects of local vapor depletion and latent heat release. The simulations with the simplified model show that the droplet size distribution becomes broader in the course of time and resembles a Gaussian distribution. This result is supported by a theoretical analysis which relates the droplet surface area distribution to the dispersion of droplets in the turbulent flow. Although the droplet growth is stabilized by vapor depletion and latent heat release in the two-way coupled model, the calculated droplet size distributions are still very broad.

The work in this chapter has been submitted in revised form as: R.S.R. Sidin, R.H.A. IJzermans, and M.W. Reeks, "A Lagrangian approach to droplet condensation in atmospheric clouds" to Physics of Fluids (2009).

\subsection{Introduction}

Despite being a familiar phenomenon, the development of rain showers is not yet completely understood and therefore subject to many studies (see, e.g., [104] and references therein). In general terms, three stages can be distinguished in the process of rain drop formation. First, droplets are generated by heterogeneous nucleation of water vapor on sub-micron sized aerosols which act as cloud condensation nuclei 
(CCNs). Although it has been speculated that very large condensation nuclei may exist in a cloud [19], [20], it is generally believed that CCNs have a size of the order of $r_{n}=10^{-7} m$ [90], [101], so that droplets start off at sub-micron sizes. If the conditions allow, the droplets grow to a diameter of millimeters in a subsequent stage, in which droplet inertia plays a dominant role. Finally, the droplets are large enough to fall, under the influence of gravity, through the cloud and reach the ground.

The growth process of droplets from sub-micron scale to millimeter scale is due to condensation (i.e., impingement of vapor molecules onto a droplet) and due to coalescence of droplets. While the condensation process depends mainly on local thermodynamic quantities such as temperature and supersaturation, the coalescence process is related to the inertia of droplets. In order to determine for which droplet sizes coalescence is important, it is instructive to look at the Stokes number St, a measure for the droplet inertia compared to the momentum of the smallest scales of turbulence [24]. Here, it is conveniently expressed as:

$$
\mathrm{St}=\frac{2}{9}\left(\frac{\rho_{b}}{\rho}\right)\left(\frac{r_{n}}{\eta_{k}}\right)^{2}
$$

where $\rho_{b}$ denotes the bulk density of the water in the droplet, $r_{n}$ is the radius of the $n^{\text {th }}$ droplet in a cloud, $\rho$ is the density of the surrounding mixture of vapor and air and $\eta_{k}$ is the Kolmogorov length scale. In systems like atmospheric clouds, typical values of the Kolmogorov length, velocity and time scales are respectively: $\eta_{k}=10^{-3} \mathrm{~m}, v_{k}=$ $0.025 \mathrm{~m} / \mathrm{s}$, and $\tau_{k}=0.04 \mathrm{~s}$ [101]. Hence, for droplets of size $10 \mu \mathrm{m}$ and a ratio between the bulk density of the water droplet and the density of the surrounding moist air of $\rho_{b} / \rho=10^{3}$, the Stokes number is approximately 0.02 . The settling velocity $v_{s}$ of these droplets in still air under the action of gravity (with acceleration $g=9.81 \mathrm{~m} / \mathrm{s}^{2}$ ) and drag forces is: $v_{s}=\mathrm{St} \tau_{k} g=0.008 \mathrm{~m} / \mathrm{s}$, which is considerably smaller than the Kolmogorov velocity scale. Therefore, droplets with radius smaller than $\sim 10 \mu \mathrm{m}$ can be expected to closely follow the turbulent flow in an atmospheric cloud, whereas inertia effects become important only for larger droplets. In conclusion, it is clear that condensation must be the dominant mechanism for the initial growth of droplets of radii between $10^{-7} \mathrm{~m}$ and $10^{-5} \mathrm{~m}$, whereas coalescence becomes important for the growth of droplets with radii larger than $10^{-5} \mathrm{~m}$.

To predict the variation in droplet size in the coalescence stage of growth, a reliable and accurate description of the droplet size distribution at the onset of this stage is necessary. In particular, the broadness of the size distribution may have a profound influence on the coalescence rate of droplets, and thus on the time scales involved in the initiation of rain. Indeed, Bec et al. [12] have found that the collision rates in a turbulent flow between polydisperse droplets can be considerably higher than between monodisperse droplets. For the current investigation, this is the main motivation to study the evolution of the droplet size distribution during the process of condensation in a model-system that simulates atmospheric clouds. 
One of the first theoretical studies on droplet condensation in clouds was published by Twomey in 1959 [123], [104]. His theory is based upon the assumption that a group of droplets may rise through a cloud in a parcel of air and remain together for a long time. As the Knudsen numbers associated with droplets in atmospheric clouds are relatively large $(K n>1)$, the growth of droplets takes place in the diffusion controlled regime [39], so that the approximation $\mathrm{d} r_{n} / \mathrm{d} t=\tilde{G} s / r_{n}$ is valid. Here, $\tilde{G}$ is a constant and $s$ is the local supersaturation [104], defined as:

$$
s \equiv S-1,
$$

with $S$ denoting the saturation ratio (see Eq. (1.1)). As the growth rate is inversely proportional to $r_{n}$, small droplets grow faster than large droplets, and therefore, the droplet size distribution is expected to become narrower as the mean droplet radius increases. This prediction is, however, not in agreement with experimental evidence, which suggests that the droplet size distributions in atmospheric clouds are broad, even when the droplets are of micrometer sizes (see, e.g., [11], [19]).

It is noted that some numerical and analytical studies on condensation in clouds have been published in recent years, also. Vaillancourt et al. [124], [125] carried out a direct numerical simulation (DNS) of condensation in a turbulent flow, thereby including a remarkable number of physical phenomena relevant for atmospheric clouds. Celani et al. ([17], [18]) recognized that, in addition to a turbulent velocity field, the supersaturation and temperature fields are unsteady and spatially inhomogeneous. Using a two-dimensional DNS, they solved an energy balance for the temperature field and a liquid-vapor mass balance for the supersaturation field simultaneously with the Navier-Stokes equations. By employing this Eulerian-Lagrangian model they were able to show that the droplet size in a cumulus cloud may be very much varied, with the largest droplets situated in regions where the supersaturation is the highest.

All of these numerical simulations are understandably restricted by computational limitations, since real atmospheric clouds are associated with excessively large Reynolds numbers and large separations of length scales. For example, performing a DNS of the turbulent flow in a cloud with an integral length scale $L_{0}=100 \mathrm{~m}$ and $\eta_{k}=10^{-3} \mathrm{~m}$ would require at least $\left(L_{0} / \eta_{k}\right)^{3}=10^{15}$ grid cells, with billions of droplets that need to be traced. Evidently, such requirements are beyond the capabilities of modern computers. In order to circumvent this problem, Vaillancourt et al. [125] focussed on the smallest scales of turbulence and chose a calculation domain of $(0.1 \mathrm{~m})^{3}$. Celani et al. [18], on the other hand, did include the largest scales in the their DNS of turbulence, but they were not able to resolve the smallest scales, despite the two-dimensionality of their model. Nonetheless it is important to note that the droplet size distribution on millimeter scales is affected by both the large and small scales of the turbulence.

As Celani et al. [17] point out, large scale motions can transport individual droplets over large variations in altitude, which results in different droplet sizes for different 
droplet trajectories. Small scale turbulent fluctuations can mix droplets of different sizes. Both phenomena together result in a broad droplet size distribution locally, which is essential for the coalescence mechanism to be enhanced.

The condensation of droplets in atmospheric clouds is a very complex process. Condensing droplets release latent heat, thereby increasing the temperature of the surrounding mixture of dry air and water vapor. Simultaneously, the droplets are transported by a turbulent flow field over several hundreds of meters, which itself is affected by the condensation processes taking place on micro scales. A further complication involves the expansion of an air parcel as it rises, thereby inducing non-zero gradients in the density, pressure and temperature fields of the mixture. Furthermore, many other phenomena take place in a cloud, such as additional temperature differences due to solar radiation (the higher parts of a cloud receive more light from the sun than the lower parts) and due to various chemical processes [90], [124], [11]. Given this rich interaction between various processes, the development of a complete physical model, which takes into account all chemical, and thermo-hydraulic phenomena occurring in actual atmospheric clouds, is an extremely challenging task. It is noted, however, that a complete model is less appropriate if one desires to isolate the influence of a specific phenomenon, which, in this specific investigation, is the role of turbulence in the cloud condensation process. In this regard, the present investigation assumes that the velocity field of the air/vapor mixture in an atmospheric cloud corresponds to a statistically stationary homogeneous isotropic turbulent flow in three dimensions, independent of the presence of the condensing droplets. This is the main assumption here, which conveniently allows the turbulent flow field to be simulated by means of a kinematic simulation (KS) employing random Fourier modes [55]. This approach has the major advantage that the flow model encompasses all turbulent length scales, from the integral scales to the Kolmogorov scales. Of course, the presence of condensing droplets in actual clouds is bound to influence the fluid dynamics within the system, e.g., by increasing the ascent rate of cloud parcels due to increased buoyancy stemming from latent heat release. Incorporation of such effects in the current model is, however, not easily achieved, nor is it desired to do so in view of the specific research questions addressed here.

In the present chapter, a fully Lagrangian numerical approach is proposed to investigate the condensation of droplets in model-systems, representing atmospheric clouds. Using the KS-flow field, the trajectories of a limited number of individual droplets, contained inside a small sampling space, are calculated backward in time in order to determine their past trajectories. Subsequently, the size of each droplet along its trajectory is then calculated forward in time. This approach efficiently produces a detailed local size distribution without the necessity of integrating the trajectories of billions of droplets individually, which would have been the case if the usual strategy of following droplets which are uniformly distributed over the entire domain had 
been adopted.

The predictions obtained with the proposed Lagrangian condensation model reveal that the droplet size distribution becomes broader in the course of time due to turbulent dispersion of droplets, both on large scales and on small scales. Moreover, it is shown that it is essential to take this entire range of scales into account: excluding the largest scales, for instance, does not result in a very broad spectrum of droplet sizes, since all droplets in the simulation experience approximately the same supersaturation (a result found by [124], [125] and [40]). Not taking into account the smallest scales does not show turbulent mixing of droplets of different sizes on the smallest scales ([18]), an effect which is vital for the enhanced coalescence mechanism. Finally, it is demonstrated that although the effects of vapor depletion and the release of latent heat slow down the droplet growth, they do not qualitatively alter the broadening of the droplet size distribution at the smallest scales.

This chapter is organized as follows. In the following section, the physical and mathematical models describing the turbulent velocity field and the condensation process are discussed. Two approaches are proposed for modelling the condensation process: a two-way coupled model in which the droplet growth exerts a feed-back on the temperature and supersaturation fields, and a simplified model in which this feed-back is neglected. Section 6.3 is devoted to the results of the statistics of droplet dispersion in the turbulent flow field. Subsequently, results are presented for the evolution of droplet size distributions, using either the simplified condensation model, or the two-way coupled model. Finally, the conclusions are formulated in the last section.

\subsection{Physical-mathematical model}

\subsubsection{Flow field composed of random Fourier modes and related droplet trajectories}

The flow of the air/vapor mixture is prescribed by a synthetic velocity field $\mathbf{u}(\mathbf{x}, t)$ composed of $M$ random Fourier modes [55]:

$$
\mathbf{u}(\mathbf{x}, t)=\sum_{m=1}^{M}\left[\mathbf{A}_{m} \cos \left(\mathbf{k}_{m} \cdot \mathbf{x}+\omega_{m} t\right)+\mathbf{B}_{m} \sin \left(\mathbf{k}_{m} \cdot \mathbf{x}+\omega_{m} t\right)\right],
$$

where $\mathbf{A}_{m}$ and $\mathbf{B}_{m}$ are orthogonal to $\mathbf{k}_{m}$, so that an incompressible flow field is obtained, which varies smoothly in both space and time. Such synthetic turbulent flow fields are frequently used to study the dispersion of particles in turbulence, e.g., [81], [113].

The wave numbers $\mathbf{k}_{m}$, amplitude coefficients $\mathbf{A}_{m}, \mathbf{B}_{m}$ and frequencies $\omega_{m}$ are all random numbers, generated in similar fashion as described in [81]. The first step is 
to adopt an energy spectrum, which characterizes the energy distribution amongst the various scales of turbulence. In this investigation, the wave vectors $\mathbf{k}_{m}$ are chosen from a model energy spectrum given by [87]:

$$
\begin{aligned}
& E(k)=\alpha k^{-5 / 3} f_{L}\left(k L_{0}\right) f_{\eta}\left(k \eta_{k}\right), k_{1} \leq k \leq k_{M} \\
& E(k)=0, \text { otherwise, }
\end{aligned}
$$

where $k \equiv\|\mathbf{k}\|$, and $L_{0}$ denotes the integral length scale. The function $f_{L}\left(k L_{0}\right)$ is a correction to the standard $-5 / 3$-spectrum that incorporates the contribution of the largest scales of motion, and is given by:

$$
f_{L}\left(k L_{0}\right)=\left[\frac{k L_{0}}{\sqrt{\left(k L_{0}\right)^{2}+C_{L}}}\right]^{5 / 3+p_{0}},
$$

with: $C_{L}=6.78$, and: $p_{0}=2$. Similarly, the function $f_{\eta}\left(k \eta_{k}\right)$ incorporates the contribution of the eddies in the dissipative range, and is given by:

$$
f_{\eta}\left(k \eta_{k}\right)=\exp \left(-\beta\left\{\left[\left(k \eta_{k}\right)^{4}+C_{\eta}^{4}\right]^{1 / 4}-C_{\eta}\right\}\right),
$$

with $C_{\eta}=0.40$ and $\beta=5$.2. The factor $\alpha$ contained in the energy spectrum is related to the energy dissipation rate $\epsilon$ by $\alpha \propto \epsilon^{2 / 3}$, and is obtained from the condition:

$$
\int_{0}^{\infty} E(k) d k=\frac{3}{2} U_{0}^{2},
$$

where $U_{0}$ is a specified reference velocity, taken as $U_{0}=1 \mathrm{~m} / \mathrm{s}$. Figure 6.1 shows the resulting energy spectrum which is used to generate the velocity field in the present investigation.

The norms $k_{m} \equiv\left\|\mathbf{k}_{m}\right\|$ are distributed in logarithmic fashion between the wave numbers associated with the maximum length scale $L_{\max }$ and the Kolmogorov length scale $\eta_{k}$ :

$$
k_{1}=\frac{2 \pi}{L_{\max }}, \quad k_{M}=\frac{2 \pi}{\eta_{k}} ; \quad k_{m}=k_{1}\left(\frac{k_{M}}{k_{1}}\right)^{\frac{m-1}{M-1}} \text { for } 1<m<M .
$$

The maximum length scale $L_{\max }$ must be larger than $L_{0}$, and its value is set equal to $5 L_{0}$ in this study. The wave numbers $\mathbf{k}_{m}$ are determined by setting: $\mathbf{k}_{m}=k_{m} \mathbf{e}_{m}$, where $\mathbf{e}_{m}$ is a unit vector whose direction is chosen randomly. This is achieved by specifying:

$$
e_{m, 1}=\sqrt{1-h^{2}} \cos \theta, e_{m, 2}=\sqrt{1-h^{2}} \cos \theta, e_{m, 3}=h
$$




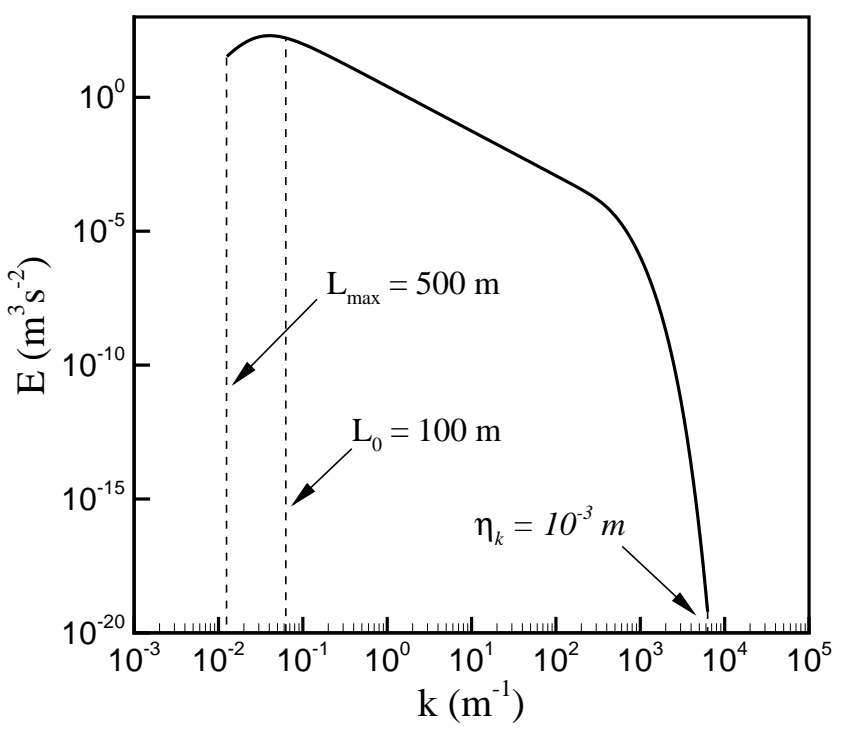

FIGURE 6.1: Turbulent energy spectrum used in the kinematically simulated flow field. The integral length scale is $L_{0}=10^{2} \mathrm{~m}$ and the Kolmogorov length scale is $\eta_{k}=$ $10^{-3} \mathrm{~m}$

for the components of $\mathbf{e}_{m}$, where the variables $h$ and $\theta$ are randomly sampled from the respective domains $\Omega_{h}=[-1,1]$, and $\Omega_{\theta}=[0,2 \pi]$, based on uniform probability distributions. The probability densities for $h$ and $\theta$ are thus given by $\operatorname{PDF}(h)=1 / 2$, and $\operatorname{PDF}(\theta)=1 / 2 \pi$, respectively.

To show that Eq. (6.9) indeed results in a uniform distribution for $\mathbf{e}_{m}$ on the unit sphere, the following analysis is carried out. First, it is recognized that Eq. (6.9) maps each region $\Omega_{h, \theta} \in \Omega_{h} \times \Omega_{\theta}$ uniquely to a region $\Omega_{\mathrm{e}}$ on the surface of the unit sphere. The probability density $\operatorname{PDF}\left(\mathbf{e}_{m}\right)$ associated with $\mathbf{e}_{m}$ satisfies the following relationship on $\Omega_{\mathrm{e}}$ :

$$
\int_{\Omega_{\mathrm{e}}} P D F\left(\mathbf{e}_{m}\right) d e_{m, 1} d e_{m, 2} d e_{m, 3}=\int_{\Omega_{h, \theta}} P D F(h) P D F(\theta) d h d \theta, \forall \Omega_{\mathbf{e}} .
$$

From vector-calculus it follows that also:

$$
\int_{\Omega_{\mathrm{e}}} P D F\left(\mathbf{e}_{m}\right) d e_{m, 1} d e_{m, 2} d e_{m, 3}=\int_{\Omega_{h, \theta}} P D F\left(\mathbf{e}_{m}(h, \theta)\right)\left\|\frac{\partial \mathbf{e}_{m}}{\partial \theta} \times \frac{\partial \mathbf{e}_{m}}{\partial h}\right\| d h d \theta, \forall \Omega_{\mathbf{e}} .
$$

Using Eq. (6.9), it is derived that

$$
\left\|\frac{\partial \mathbf{e}_{m}}{\partial \theta} \times \frac{\partial \mathbf{e}_{m}}{\partial h}\right\|=1
$$


which means that:

$$
\operatorname{PDF}\left(\mathbf{e}_{m}\right)=\operatorname{PDF}(h) P D F(\theta)=1 / 4 \pi,
$$

because Eq. (6.10) and (6.11) must be satisfied for arbitrary $\Omega_{\mathrm{e}}$. Thus it is established that the mapping in Eq. (6.9) indeed results in a uniform distribution of $\mathbf{e}_{m}$ on the unit sphere.

In order to ensure that the velocity field satisfies the energy spectrum, the norms of the amplitude coefficients $\mathbf{A}_{m}$ and $\mathbf{B}_{m}$ are determined from:

$$
\left\|\mathbf{A}_{m}\right\|^{2}=\left\|\mathbf{B}_{m}\right\|^{2}=2 \int_{k_{m-1 / 2}}^{k_{m+1 / 2}} E(k) d k
$$

where: $k_{m \pm 1 / 2}=\left(k_{m}+k_{m \pm 1}\right) / 2$. The direction of $\mathbf{A}_{m}$ is specified by setting: $\mathbf{A}_{m}=$ $\left\|\mathbf{A}_{m}\right\| \mathbf{a}_{m}$; the unit vector $\mathbf{a}_{m}$ is given by:

$$
\mathbf{a}_{m}=\frac{\mathbf{t}_{m} \times \mathbf{k}_{m}}{\left\|\mathbf{t}_{m} \times \mathbf{k}_{m}\right\|}
$$

where $\mathbf{t}_{m}$ is a randomly directed unit vector. Determination of the amplitude coefficients $\mathbf{B}_{m}$ proceeds in a similar way as outlined for $\mathbf{A}_{m}$.

The velocity field is completed by specifying the angular frequencies $\omega_{m}$, which are chosen to be proportional to the eddy-turnover time associated with the $m$-th Fourier mode [35]:

$$
\omega_{m}=\lambda \sqrt{k_{m}^{3} E\left(k_{m}\right)},
$$

where $\lambda$ is the so-called unsteadiness parameter which is generally taken to be $0 \leq$ $\lambda \leq 1$. In this investigation its value is set to $\lambda=1$.

Droplets are assumed to be advected by the turbulent flow as passive tracers. This is a valid approximation in the present research, since the focus is primarily on droplets with sizes $10^{-7} m<r_{n}<10^{-5} \mathrm{~m}$, for which the Stokes number based on the Kolmogorov scales is $0.2 \times 10^{-5}<\mathrm{St}<0.02$, and the ratio between the settling velocity and the Kolmogorov velocity is $0.3 \times 10^{-4}<v_{s} / v_{k}<0.3$. Also, Brownian motion is neglected, since Brownian diffusion is much smaller than the turbulent diffusion for the flow fields that are simulated in this investigation. Thus, the position $\mathbf{x}_{n}(t)$ of a droplet in the course of time $t$ is given by:

$$
\frac{\mathrm{d} \mathbf{x}_{n}}{\mathrm{~d} t}=\mathbf{u}\left(\mathbf{x}_{n}(t), t\right)
$$

with $\mathbf{u}\left(\mathbf{x}_{n}(t), t\right)$ specified by Eq. (6.3). 


\subsubsection{Two-way coupled droplet condensation model}

The average number density of droplets in atmospheric clouds is much lower than the number densities observed in condensing nozzle flows. In condensing nozzle flows the inter-droplet separation distances are typically $O\left(10^{-6} \mathrm{~m}\right)$, whereas in atmospheric clouds, the average inter-droplet separation distance is $O\left(10^{-3} \mathrm{~m}\right)$, which is comparable to the typical Kolmogorov length scales of atmospheric turbulence. It is therefore not possible to use a continuum modelling approach to describe the evolution of the droplet size distribution in systems where the details on such small scales are of interest. As a consequence, a discrete particle approach is adopted, in which the condensation of droplets is tracked in Lagrangian fashion along its trajectory. In contrast to the usual practice in discrete particle models, the present method is fully Lagrangian, because the fluid velocity does not need to be solved on a fixed Eulerian grid, since it can be computed by means of Eq. (6.3) at any point in time, at the position of each individual droplet.

To derive the Lagrangian condensation model, a single droplet of mass $m_{n}$ is considered, which moves in a turbulent velocity field $\mathbf{u}(\mathbf{x}, t)$. The droplet is contained within an air/vapor parcel of volume $V_{c}$, which is assumed to be much larger than the droplet's volume, but small enough so that all molecules in the parcel remain together for a long time, i.e., the diffusive mass and energy transport at the outer surface of the parcel is neglected. First, an expression is introduced for the supersaturation in the parcel based on a mass balance, and subsequently, the equation for the temperature variation following from an energy balance is derived. Furthermore, the temperature and vapor mass density are assumed to remain uniform over the entire parcel.

The volume of the parcel $V_{c}$ can be considered as the volume of air and vapor available per droplet. It is therefore related to the droplet number density $N_{l}$ as:

$$
V_{c}=N_{l}^{-1}
$$

If the mass of air inside the parcel is denoted by $m_{a}$, the mass of vapor by $m_{v}$, and the mass of the droplet by $m_{n}$, a mass balance yields:

$$
\frac{\mathrm{d} m_{v}}{\mathrm{~d} t}=-\frac{\mathrm{d} m_{n}}{\mathrm{~d} t}
$$

as only vapor can be transformed into liquid and vice versa. Upon neglecting the droplet's volume compared to $V_{c}$, the partial vapor density $\rho_{v}$ in the air/vapor mixture can be determined by:

$$
\rho_{v}=\frac{m_{v}}{V_{c}}
$$

and its time derivative along the droplet's trajectory is:

$$
\frac{\mathrm{d} \rho_{v}}{\mathrm{~d} t}=\frac{1}{V_{c}} \frac{\mathrm{d} m_{v}}{\mathrm{~d} t}-\frac{m_{v}}{V_{c}^{2}} \frac{\mathrm{d} V_{c}}{\mathrm{~d} t}=-\frac{1}{V_{c}} \frac{\mathrm{d} m_{n}}{\mathrm{~d} t}-\frac{m_{v}}{V_{c}^{2}} \frac{\mathrm{d} V_{c}}{\mathrm{~d} t} .
$$


The rate-of-change of $V_{c}$ is not necessarily zero because the volume $V_{c}$ may expand (shrink) when it is transported to higher (lower) altitudes. Since in atmospheric clouds $m_{a} \gg m_{v}+m_{n}$ [101], the evolution of $V_{c}$ in time can in principle be determined from $V_{c}=m_{a} / \rho_{a}$, where $\rho_{a}$ denotes the partial density of air. It can be shown that the variation of $\rho_{a}$ is so weak in atmospheric clouds that the influence of the second term on the right-hand side of Eq. (6.21) is negligible compared to the other thermodynamical effects. Therefore,

$$
\frac{\mathrm{d} V_{c}}{\mathrm{~d} t}=0
$$

and, as a consequence of Eq. (6.18), $N_{l}$ can be considered constant as well.

The law describing the condensational growth of a droplet varies considerably with the droplet's size. For very small droplets (i.e., for large Knudsen number) the growth is described by the Hertz-Knudsen law which includes the so-called Kelvin effect [90], whereas the growth rate of large droplets (i.e., for small Knudsen number) is governed by diffusion. For the sake of simplicity, the diffusional growth law is employed for all droplet sizes in the present study. Although it overestimates the droplet growth rate for the smallest droplets of size $r_{n} \lesssim 10^{-7} \mathrm{~m}$, it is accurate for the majority of the droplets in the present simulations, whose radius is generally much larger than the capillary length scale.

The diffusional growth law for a spherical droplet is [39]:

$$
\frac{\mathrm{d} m_{n}}{\mathrm{~d} t}=4 \pi r_{n}^{2} \rho_{b} \frac{\mathrm{d} r_{n}}{\mathrm{~d} t}=\dot{m}_{d}=4 \pi r_{n} D_{v, a}\left(\rho_{v}-\rho_{v}^{s}\right),
$$

where $D_{v, a}$ is the binary diffusion coefficient of water vapor in air, $\rho_{b}$ is the bulk density of water $\left(\rho_{b}=10^{3} \mathrm{~kg} / \mathrm{m}^{3}\right)$ and $\rho_{v}^{s}$ denotes the saturation vapor density, which depends on the temperature: $\rho_{v}^{s}=\rho_{v}^{s}(T)$ (see appendix A).

By writing the supersaturation $s$ of the moist air surrounding the droplet in the form:

$$
s=\frac{\rho_{v}}{\rho_{v}^{s}}-1,
$$

Eq. (6.23) can be cast as:

$$
\dot{m}_{n}=4 \pi r_{n} D_{v, a} s \rho_{v}^{s} .
$$

The rate-of-change of the supersaturation can be obtained by differentiating Eq. (6.24) with respect to time, and making use of Eq. (6.21), Eq. (6.22) and Eq. (6.25) in order to obtain:

$$
\frac{\mathrm{d} s}{\mathrm{~d} t}=-\frac{s}{\tau_{s}}-(s+1) \frac{\mathrm{d} T}{\mathrm{~d} t} \frac{1}{\rho_{v}^{s}} \frac{d \rho_{v}^{s}}{d T},
$$

where the vapor depletion time scale $\tau_{s}$ is given by:

$$
\tau_{s}=\frac{V_{c}}{4 \pi r_{n} D_{v, a}}=\frac{1}{4 \pi r_{n} D_{v, a} N_{l}} .
$$


An equation for the temperature of the moist air surrounding the droplet can be obtained by considering the conservation of energy in the volume $V_{c}$. For a system of this size, the variation of the enthalpy within the volume $V_{c}$ is negligible, and therefore the conservation of energy is given by:

$$
\frac{\mathrm{d}}{\mathrm{d} t}\left(m_{a} h_{a}+m_{v} h_{v}+m_{n} h_{n}\right)=-\left(m_{a}+m_{v}+m_{n}\right) \mathbf{u} \cdot \mathbf{e}_{z} g,
$$

where $h_{a}, h_{v}$ and $h_{n}$ denote the specific enthalpy of the air, the vapor and the droplet, respectively. Eq. (6.28) expresses the balance between the change in the energy of the parcel of moist air and the work done by gravity, as viscous dissipation and thermal heat conduction are neglected. Since the supersaturation is close to zero [101], the liquid enthalpy $h_{n}$ may be approximated by:

$$
h_{n}=h_{v}-L_{b},
$$

where $L_{b}=L_{b}(T)$ is the bulk latent heat of vaporization. By employing a caloric equation of state for a perfect gas: $h_{a}=C_{p, a} T$ and: $h_{v}=C_{p, v} T$ (with $C_{p, i}$ denoting the isobaric specific heat of substance $i$ ), in combination with Eq. (6.19) and Eq. (6.29), Eq. (6.28) may be cast into the following form:

$$
\frac{\mathrm{d} T}{\mathrm{~d} t}=\frac{\dot{m}_{d} L_{b}-\left(m_{a}+m_{v}+m_{n}\right) \mathbf{u} \cdot \mathbf{e}_{z} g}{m_{a} C_{p, a}+\left(m_{v}+m_{n}\right) C_{p, v}-m_{n} \mathrm{~d} L_{b} / \mathrm{d} T} .
$$

Since in atmospheric clouds it holds that $m_{a} C_{p, a} \gg\left(m_{v}+m_{n}\right) C_{p, v}$ and $m_{a} C_{p, a} \gg$ $m_{n} \mathrm{~d} L_{b} / \mathrm{d} T$, Eq. (6.30) may be further simplified to:

$$
\frac{\mathrm{d} T}{\mathrm{~d} t}=\frac{\dot{m}_{d} L_{b} N_{l}}{\rho_{a} C_{p, a}}-\Gamma \mathbf{u} \cdot \mathbf{e}_{z}
$$

where the factor $\Gamma$ is the so-called adiabatic lapse rate [101], given by:

$$
\Gamma=\frac{g}{C_{p, a}}
$$

The two-way coupled system is now closed, and it can be solved along the trajectory of a droplet in the course of time. Specifically, Eq. (6.26) and Eq. (6.31) for the supersaturation and temperature of the moist air surrounding the droplet, respectively, and Eq. (6.25) for the rate-of-change of the droplet mass, need to be solved, using some initial conditions for $s(t=0), T(t=0)$ and $m_{n}(t=0)$. This system of ODE's is augmented by Eq. (6.27) for the saturation relaxation time $\tau_{s}$, as well as the expressions for the quantities $D_{v, a}(p, T), L_{b}(T)$ and $\rho_{v}^{s}(T)$ and $C_{p, i}$, which are all given in Appendix A. Finally, the parameters $\rho_{a}$ and $N_{l}$ can be chosen freely depending on the problem at hand. 
The model just presented is referred to as the "Two-way coupled condensation model'. It should be noted that Eq. (6.26) is actually very similar to the expression used by Twomey to calculate the variation in the supersaturation in [123]. Although the effect of latent heat release has been excluded in that particular investigation, recent work by Celani et al. [17] does take it into account by means of an appropriate sink term in the balance equation for the supersaturation. The main difference between the current 'Two-way coupled condensation model' and the one in [17] is that the latter uses an Eulerian formulation to describe the evolution in $s$, whereas here, a fully Lagrangian approach is employed.

\subsubsection{Simplified droplet condensation model}

As can be noted from Eq. (6.26) and Eq. (6.31), the supersaturation changes due to three effects: adiabatic cooling, vapor depletion (i.e., the finiteness of $V_{c}$ ) and the release of latent heat by the condensing droplet. The latent heat release and the vapor depletion term tend to slow down the growth of droplets and thus have a stabilizing effect on the development of the droplet size distribution in $r_{n}$-space. Adiabatic cooling, however, is the only mechanism capable of increasing the supersaturation when a droplet is growing. It can therefore be seen as the only stimulating effect on the condensation process. Indeed, adiabatic cooling is widely believed to be the main source of supersaturation, and therefore of the condensational growth of droplets in a cloud [104], [17].

In order to isolate the effect of adiabatic cooling, a simplified condensation model is proposed, in which the effects of vapor depletion and latent heat release are neglected. This approach has two advantages: firstly, it allows one to determine the influence of adiabatic cooling on the development of the droplet size distribution, without the results being obscured by secondary effects. Secondly, in a subsequent stage, the results obtained with the simplified condensation model can be compared with the results from the two-way coupled condensation model. Any differences between the results can unambiguously be related to the feed-back of the droplet growth on the temperature and supersaturation fields.

The simplified condensation model follows readily from the two-way coupled condensation model presented in section 6.2.2. If latent heat release is neglected, Eq. (6.31) reduces to:

$$
\frac{\mathrm{d} T}{\mathrm{~d} t}=-\Gamma \mathbf{u} \cdot \mathbf{e}_{z}
$$

Similarly, if the effect of vapor depletion is neglected, all terms involving the finiteness of the volume $V_{c}$ are zero and Eq. (6.26) becomes:

$$
\frac{\mathrm{d} s}{\mathrm{~d} t}=-(s+1) \frac{\mathrm{d} T}{\mathrm{~d} t} \frac{1}{\rho_{v}^{s}} \frac{d \rho_{v}^{s}}{d T}=(s+1) \Gamma \mathbf{u} \cdot \mathbf{e}_{z} \frac{1}{\rho_{v}^{s}} \frac{d \rho_{v}^{s}}{d T} .
$$

Finally, the droplet growth law is still given by Eq. (6.23). 
This model, which is similar to the model employed by [17], is referred to as the 'Simplified condensation model', and accounts for condensation in a prescribed temperature field resulting from adiabatic expansion cooling.

\subsection{Results}

\subsubsection{Statistics of droplet dispersion}

It is instructive to discuss some statistics of the one-droplet and two-droplet dispersion in the turbulent flow described by Eq. (6.3). Here and in the following, the integral length scale of the flow is set equal to $L_{0}=10^{2} \mathrm{~m}$, and the Kolmogorov length scale to $\eta_{k}=10^{-3} \mathrm{~m}$.

In this flow field, the trajectories of droplets in the course of time are determined by integrating Eq. (6.17) numerically, using a fourth-order Runge-Kutta scheme. The numerical time step in all simulations presented here is fixed at $\Delta t=0.1 / \omega_{N}$, so that the motion of a droplet through the smallest turbulent eddies is resolved with sufficient accuracy. Convergence tests have been carried out with smaller values of $\Delta t$, and the results for the dispersion or the condensation of droplets were not significantly different.

First, the one-droplet dispersion is investigated by calculating the trajectories of 500 droplets in five different realizations of the flow field. Each droplet is released at a random point $\mathbf{x}_{n}(0)$ and traced for a time $0 \leq t \leq 10^{4} \mathrm{~s}$, during which the distance to the point of injection $\left\|\mathbf{x}_{n}(t)-\mathbf{x}_{n}(0)\right\|$ is calculated. The square root of the squared distance averaged over all droplets $\left\langle\left\|\mathbf{x}_{n}(t)-\mathbf{x}_{n}(0)\right\|^{2}\right\rangle^{1 / 2}$ is plotted in Figure 6.2.a as a function of time. The result shows that the droplets in an atmospheric cloud can be transported over distances of the order of $100 \mathrm{~m}$ within a time span of $100 \mathrm{~s}$. In addition, it is clear that $\left\langle\left\|\mathbf{x}_{n}(t)-\mathbf{x}_{n}(0)\right\|^{2}\right\rangle^{1 / 2} \propto t$ for small values of $t$, and $\left\langle\| \mathbf{x}_{n}(t)-\right.$ $\left.\mathbf{x}_{n}(0) \|^{2}\right\rangle^{1 / 2} \propto t^{1 / 2}$ for large values of $t$, which is perfectly in agreement with Taylor's famous prediction of one-particle dispersion in a turbulent flow [121].

The statistics of the two-droplet dispersion are determined by releasing 500 pairs of droplets at an initial separation $d_{0}$ in five different realizations of the flow. The initial distance is chosen as the Kolmogorov length scale, $d_{0}=\eta_{k}$, and the direction of the inter-particle separation vector is selected randomly in three dimensions. The trajectory of each droplet in a droplet pair is calculated from Eq. (6.17), and at each time step the distance between the droplets $d(t)$ is measured. Averaging over all droplet pairs then results in $\left\langle d^{2}(t)\right\rangle^{1 / 2}$, which is plotted in Figure 6.2.b as a function of time. The separation distance is apparently proportional to $t$ for small values of $t$, and proportional to $t^{1 / 2}$ for very large values of $t$. In the intermediate time range, the separation distance in a turbulent flow should be proportional to $t^{3 / 2}$ when the separation distance is of the order of the size of the eddies in the turbulent inertial range, as the classical theory by Richardson predicts [94]. Due to the limited statistics, it is not 


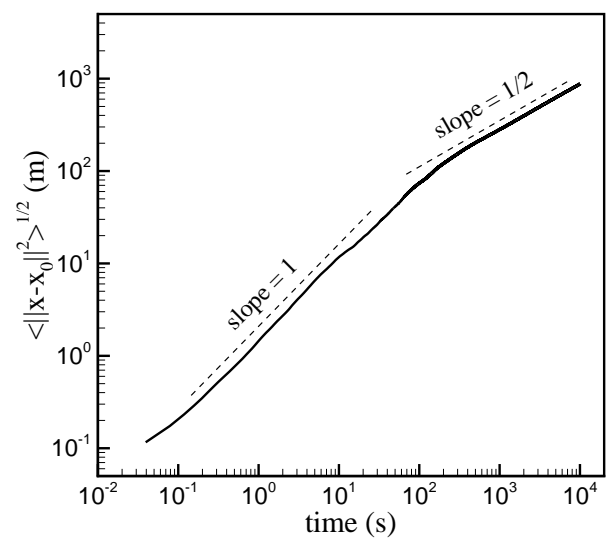

(a)

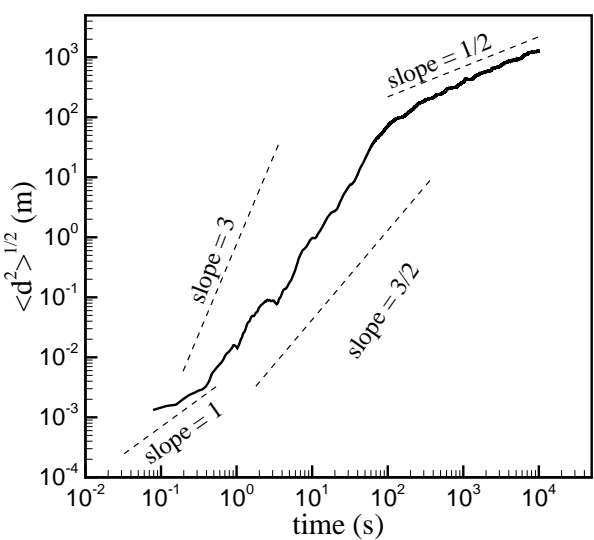

(b)

FIGURE 6.2: (a) Lagrangian one-particle statistics, based on 500 droplets in 5 different realizations of the flow field composed of 200 random Fourier modes; (b) Lagrangian two-particle statistics, based on 500 droplet pairs in 5 different realizations of the flow field composed of 200 random Fourier modes. The initial inter-particle separation distance is equal to the Kolmogorov length scale: $d_{0}=\eta_{K}=10^{-3} \mathrm{~m}$.

immediately clear whether or not the current kinematic simulation produces exactly that behavior, but in spite of this, a range is observed in which $\left\langle d^{2}(t)\right\rangle^{1 / 2} \propto t^{p}$, with $p$ somewhere between $3 / 2<p<3$, in general agreement with Thomson \& Devenish [122] who found $p \simeq 2.3$. It is well known that KS are not able to fully reproduce all details of a real turbulent flow field, such as the sweeping effect of large eddies on smaller ones. Regardless of the in itself interesting question whether a kinematic simulation is able to produce Richardson's law (see, e.g., [4], however, [81]), it is clear from Figure 6.2.b that two droplets which are initially separated by a small distance may end up in completely different regions of the flow field after a sufficiently long time. Vice versa, since the equation of motion Eq. (6.17) is reversible, it also holds that two droplets which are nucleated at two distantly separated positions, may end up very close to one another at some instant of time. This concept turns out to be essential for the explanation of the results obtained with the current condensation models.

\subsubsection{Results for simplified condensation model}

In the present section the results obtained for the simplified condensation model are discussed. The size and position of a droplet at a final time $t_{e}$ are obtained in three steps. First, the final position $\mathbf{x}_{n, e}=\left(x_{e}, y_{e}, z_{e}\right)^{T}$ is specified by choosing an altitude $z_{e}$, whereas the horizontal coordinate $\left(x_{e}, y_{e}\right)$ is chosen randomly in a square with 
sides of length $L$.

Second, the trajectory of the droplet is calculated backward in time (i.e., from $t=t_{e}$ to $t=0$ ) from the final position. In this step, the position of the droplet follows from:

$$
\frac{\mathrm{d} \mathbf{x}_{n}}{\mathrm{~d} \tau}=-\mathbf{u}\left(\mathbf{x}_{n}(\tau), \tau\right) \quad ; \quad \mathbf{x}_{n}(\tau=0)=\mathbf{x}_{n}\left(t=t_{e}\right)=\mathbf{x}_{n, e},
$$

with $\tau=t_{e}-t$ running from 0 to $t_{e}$, and $\mathbf{u}\left(\mathbf{x}_{n}(\tau), \tau\right)$ prescribed by Eq. (6.3).

Third and finally, the droplet growth is determined forward in time along its trajectory. Eq. (6.23) for the droplet mass $m_{n}(t)$ is solved forward in time, starting from the initial condition $m_{n}(0)$. All droplets are supposed to have the same size $r_{0}=10^{-7} \mathrm{~m}$ initially, so that $m_{n}(0)=(4 \pi / 3) \rho_{n} r_{0}^{3}$.

Using this procedure, a total of $N_{l, t o t}=16,000$ droplets is traced through the flow field in each simulation. This procedure efficiently produces droplet size statistics within the sampling area $x \in[0, L], y \in[0, L]$ and $z=z_{e}$ at time $t=t_{e}$, whilst it avoids the necessity to follow a huge number of particles uniformly distributed over the entire domain, like in traditional Eulerian-Lagrangian methods.

It is noted that for the simplified model, the local temperature and supersaturation values are obtained from a prescribed profile (see Figure 6.3), for which the data is obtained from the adiabatic cooling of a parcel of rising air. The profiles $T(z)$ and $s(z)$ are given by:

$$
T(z)=T(z=0)-\Gamma z, \quad \text { and } \quad s(z)=\frac{\rho_{v}}{\rho_{v}^{s}(T(z))}-1,
$$

where the vapor density $\rho_{v}$ is constant because its variations are much smaller than the variations in $\rho_{v}^{s}$. The vapor density is therefore set equal to its value at the earth's surface: $\rho_{v}=\rho_{v}(z=0)$. For all the simulations presented in this paper, the conditions at the earth's surface $(z=0)$ are taken as: $T(z=0)=20^{\circ} \mathrm{C}=293 \mathrm{~K}$, with a relative humidity of $50 \%(s=-0.5)$.

Given Eq. (6.23), it is obvious that a droplet may shrink to a mass of zero, if it experiences negative supersaturation for a sufficiently long time. Physically, this means that it is completely evaporated. If such happens, the droplet is eliminated from the calculation and makes no contribution to the size distribution function.

Figure 6.4 shows the droplet size distribution for $z_{e}=1350 \mathrm{~m}$ and a sampling area $L^{2}=(500 m)^{2}$, for seven different values of $t_{e}$. The distribution function is a Dirac delta function at $t_{e}=0$, and becomes broader for larger $t_{e}$. Already after $t_{e}=20 \mathrm{~s}$, some droplets have reached a size of tens of microns, which is at first glance remarkable, because the supersaturation at $z_{e}=1350 \mathrm{~m}$ is very close to zero (see Figure 6.3). The explanation of the spectral broadening lies in turbulent dispersion [17]: droplets with different histories are located in the sampling space situated at altitude $z_{e}$ at time $t_{e}$. Droplets which have been at higher altitudes have experienced higher supersaturations and thus have grown more than droplets which have been at lower altitudes. 


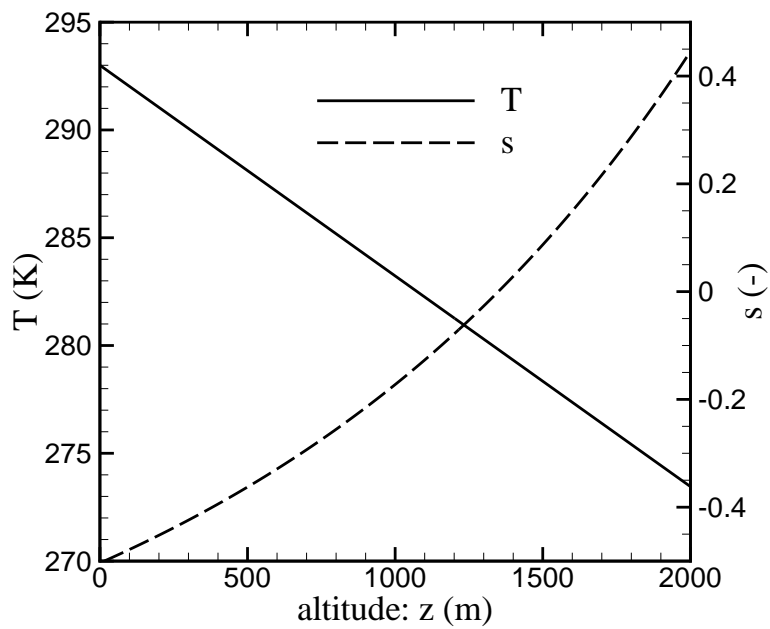

FIGURE 6.3: Temperature and supersaturation profiles as a function of altitude $z$. At the earth's surface $z=0, T=293 \mathrm{~K}$ and $s=-0.5$.

Comparing these results with those of Twomey [123], [104], it is evident that it is of fundamental importance to include turbulent dispersion in the model.

In Figure 6.5, a comparison is made of the droplet size distributions after time $t_{e}=100 \mathrm{~s}$, at five different altitudes $z_{e}$. Sampling spaces at high altitudes $z_{e}$ are predominantly populated by droplets which (on average) have experienced higher supersaturations than droplets at low altitudes (see Figure 6.3). Therefore, the mean size of droplets at high $z_{e}$ is larger than at low $z_{e}$, and this trend is clearly visible in Figure 6.5. The variance of $f\left(r_{n}\right)$, however, does not seem to be affected much by the altitude, as the droplet size distributions are relatively broad for all altitudes considered.

The fraction of evaporated droplets is determined by simply counting the number of evaporated droplets $N_{\text {l,evap }}$ in the simulations presented in Figure 6.5 and comparing them to the total number of droplets $N_{l, t o t}$ initially released in the flow. The ratio $N_{l, \text { evap }} / N_{l, t o t}$ is plotted in Figure 6.6. It is clear that more droplets evaporate at lower values of $z_{e}$, which is a consequence of the fact that the supersaturation is less at lower altitudes, and therefore, the fraction of droplets experiencing negative supersaturation is higher. The high initial evaporation rate is due to the fact that some of the droplet trajectories have a starting point $z_{d}(0)$ in a region where $s<0$ : these droplets evaporate almost immediately.

It is important to note that droplet nucleation after $t=0$ is not taken into account in the current model. In reality, however, a new droplet may grow along the trajectory of a previously evaporated droplet. The regeneration of droplets has been neglected 


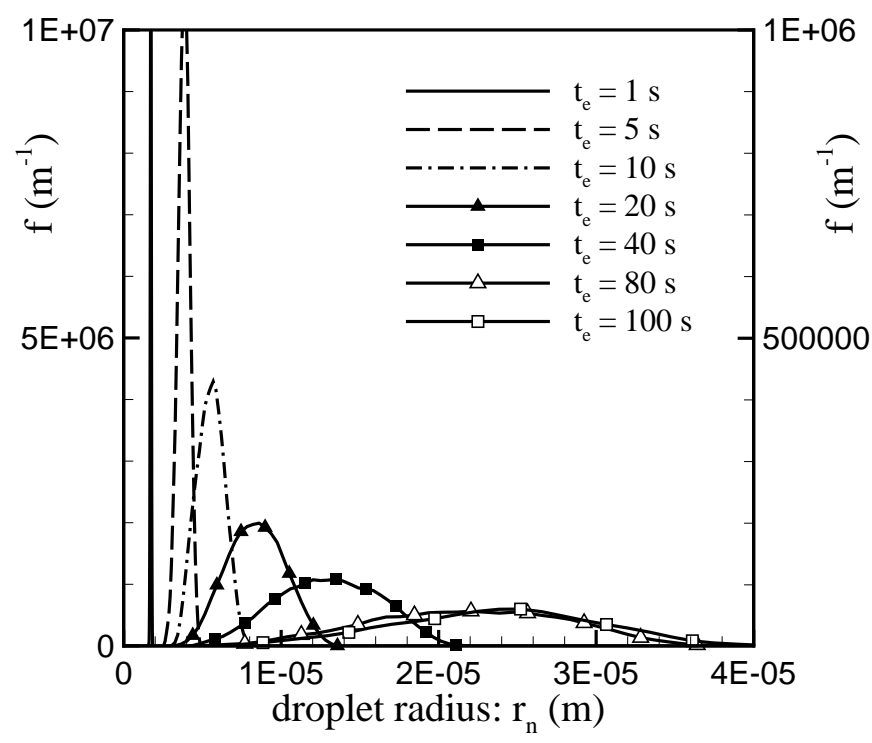

Figure 6.4: Droplet radius distribution function $f\left(r_{n}\right)$ for seven different moments in time $t_{c}$. The final altitude of droplets is $z_{e}=1350 \mathrm{~m}$, and the sampling area $L^{2}=$ $(500 m)^{2}$. The results have been obtained with the simplified condensation model.

for the sake of simplicity, but this phenomenon is likely to be present in atmospheric clouds and could eventually result in a multi-modal droplet size distribution function.

At this point it is useful to examine the relationship between the results and the relevant parameters which determine the temperature and supersaturation profiles. It follows from Eq. (6.23) that the surface of the droplet $A_{n}(t) \equiv 4 \pi r_{n}^{2}(t)$ changes as:

$$
\dot{A}_{n}=G s, \quad \text { with: } \quad A_{n}(0)=4 \pi r_{0}^{2},
$$

where $G=8 \pi D_{v, a} \rho_{v}^{s} / \rho_{n}$. Most droplets in the current simulations experience temperature variations that are so small that $G$ remains approximately constant. In addition, in the simplified condensation model, the equation for the supersaturation, Eq. (6.26), can be integrated to obtain:

$$
s(t)=s\left(z_{d}(t)\right)=A\left(z_{d}(t)-z_{r e f}\right),
$$

for a constant value of the parameter $A=\Gamma \mathrm{d}\left(\ln \rho_{v}^{s}\right) / \mathrm{d} T$, which is a reasonable approximation in the present case where the temperature variations are relatively weak. In fact, Eq. (6.38) stems from a local linearization of the adiabatic supersaturation profile shown in Figure 6.3. The value $z_{\text {ref }}$ denotes the reference altitude for which the supersaturation is zero. Eq. (6.37) can be integrated along the trajectory of a droplet 


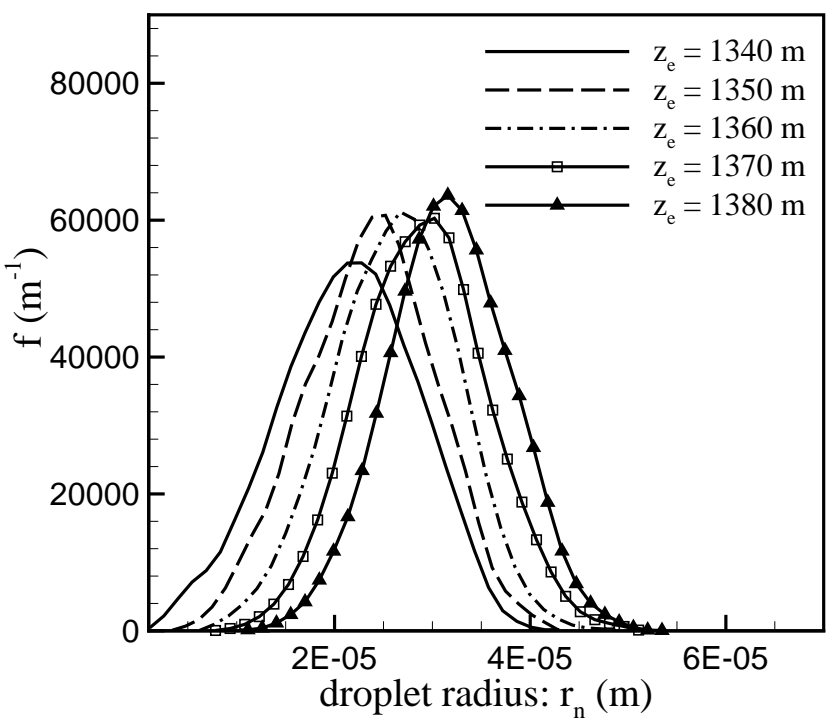

FIGURE 6.5: Droplet radius distribution function $f\left(r_{n}\right)$ for five different final altitudes of droplets $z_{e}$. The sampling time $t_{e}=100 \mathrm{~s}$, and the sampling area $L^{2}=(500 \mathrm{~m})^{2}$. The results have been obtained with the simplified condensation model.

using Eq. (6.38), and the result is:

$$
A_{n}(t)=4 \pi r_{0}^{2}+G A \zeta(t)
$$

with:

$$
\zeta(t) \equiv \int_{0}^{t}\left(z_{d}\left(t^{\prime}\right)-z_{r e f}\right) \mathrm{d} t^{\prime} .
$$

Thus, $A_{n}(t)$ and $\zeta(t)$ are proportional, and their statistics, determined by averaging over a large number of droplets, are similar:

$$
\operatorname{PDF}(\zeta)=\operatorname{PDF}\left(\frac{A_{n}-4 \pi r_{0}^{2}}{G A}\right) .
$$

In other words, one could, in principle, estimate the droplet size distribution at a given altitude $z_{e}$ by using only the statistics for the dispersion.

The PDF of $\zeta$ at $t_{e}=100 \mathrm{~s}$ is shown in Figure 6.7 for droplets which have a final position in a sampling area of size $L^{2}=(500 \mathrm{~m})^{2}$ at altitude $z_{e}=1350 \mathrm{~m}$. The same graph also includes the PDF of the mean supersaturation $s_{m}\left(t_{e}\right)$ along the trajectory 


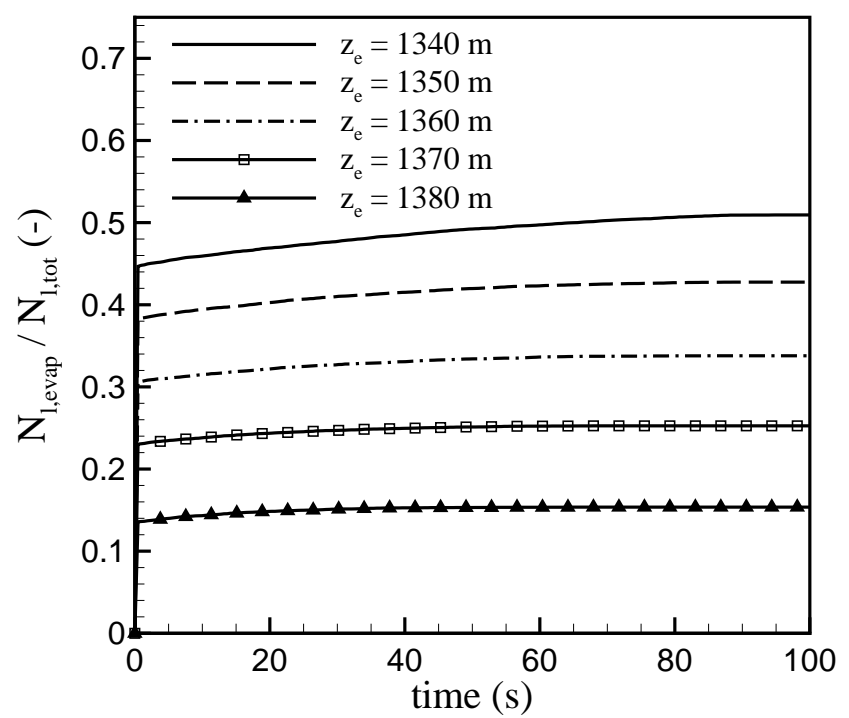

FIGURE 6.6: Number of evaporated droplets relative to the total number of droplets in the simulation as a function of time, for five different final altitudes $z_{e}$ after time $t_{e}=100 \mathrm{~s}$. The results have been obtained with the simplified condensation model with $L^{2}=(500 m)^{2}$.

of a droplet, which is defined as:

$$
s_{m}(t) \equiv \frac{1}{t} \int_{0}^{t} s\left(t^{\prime}\right) \mathrm{d} t .
$$

It is noted that all droplet trajectories have been included in generating these PDF's, i.e., including those for which droplets evaporate completely. In order to allow direct comparison, the PDFs are shown as a function of the scaled standardized variables:

$$
x_{\zeta}^{\prime} \equiv \frac{\zeta-\langle\zeta\rangle}{\sqrt{\operatorname{var}(\zeta)}}, \quad x_{s_{m}}^{\prime} \equiv \frac{s_{m}-\left\langle s_{m}\right\rangle}{\sqrt{\operatorname{var}\left(s_{m}\right)}}
$$

where $\operatorname{var}(\cdot)$ stands for the variance obtained from averaging over all droplets. A perfect agreement is observed, as is expected from the linearity of Eq. (6.38). In addition, it is noteworthy that the PDFs are symmetric, which follows from the isotropy of the flow field, and furthermore, they are almost perfectly Gaussian.

Next, the correlation between the parameter $\zeta$ and $A_{n}$ is investigated. Figures 6.8. $\mathrm{a}$ and $\mathrm{b}$ show the PDF of the normalized quantities $x_{\zeta}^{\prime}$ and $x_{A_{n}}^{\prime}$ (with $x_{A_{n}}^{\prime}$ obtained 


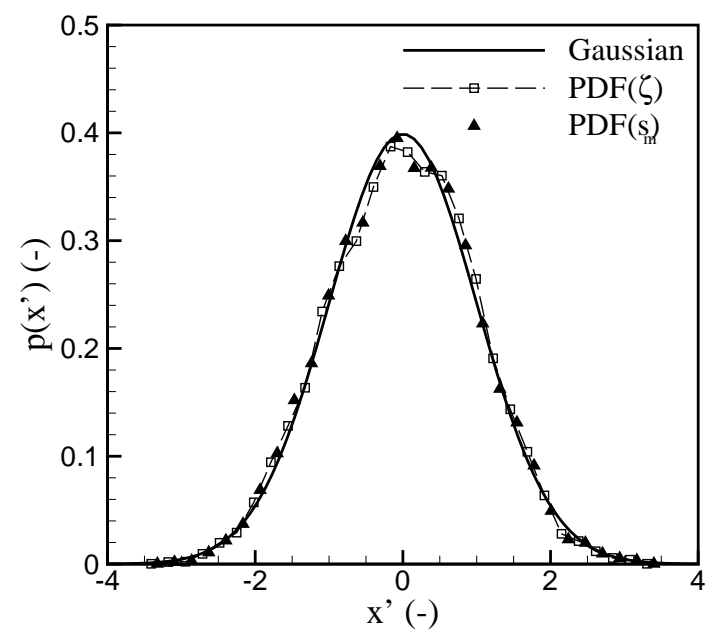

FIGURE 6.7: Standardized PDFs of $\zeta$ (see Eq. (6.40)) in the simulation (evaporated or not), and the mean supersaturation $s_{m}$ along their trajectories. For the sake of comparison, a Gaussian distribution with zero mean and unity variance is plotted as well. The sampling time $t_{e}=100 \mathrm{~s}$, the final altitude of droplets $z_{e}=1350 \mathrm{~m}$, and the sampling area $L^{2}=(500 m)^{2}$. The results have been obtained with the simplified condensation model.

analogously to $x_{\zeta}^{\prime}$ ) only for droplets that have not evaporated, for sampling altitudes $z_{e}=1350 \mathrm{~m}$ and $z_{e}=1380 \mathrm{~m}$, respectively. Both figures show a perfect agreement between $P D F\left(x_{\zeta}^{\prime}\right)$ and $P D F\left(x_{A_{n}}^{\prime}\right)$, which confirms the previous analysis presented in Eq. (6.41). From comparing Figure 6.8.a with Figure 6.8.b, it is clear that the PDF of $x_{A_{n}}^{\prime}$ is more symmetric for higher $z_{e}$. This can be explained as follows. Any asymmetry in the graphs is purely due to the evaporation of droplets because the PDF of $\zeta$ measured for all droplets (evaporated and non-evaporated) should be symmetric due to the isotropy of the turbulent flow. If $z_{e}$ increases, the number of evaporated droplets decreases (see Figure 6.6) and therefore, the standardized PDF of $A_{n}$ becomes more symmetric. Figure 6.8.a and Figure 6.8.b also show the PDF of the standardized droplet radius $x_{r_{n}}^{\prime}$, which is remarkably close to Gaussian. This is probably a coincidence since there is no evidence that the PDF of $r_{n}$ is directly governed by a Gaussian process.

The evolution of the mean and variance of the PDF of $A_{n}$ in the course of time can be estimated on the basis of the mean and the variance of the PDF of $\zeta$. Since:

$$
z_{d}(t)=z_{e}-\int_{t}^{t_{e}} w\left(t^{\prime}\right) \mathrm{d} t^{\prime}
$$




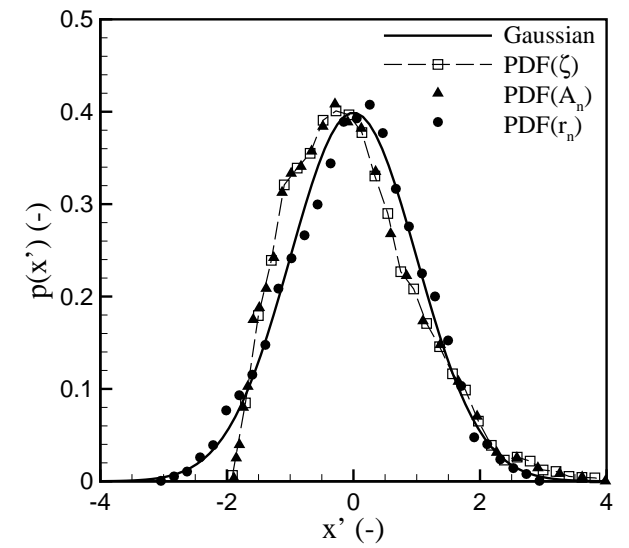

(a)

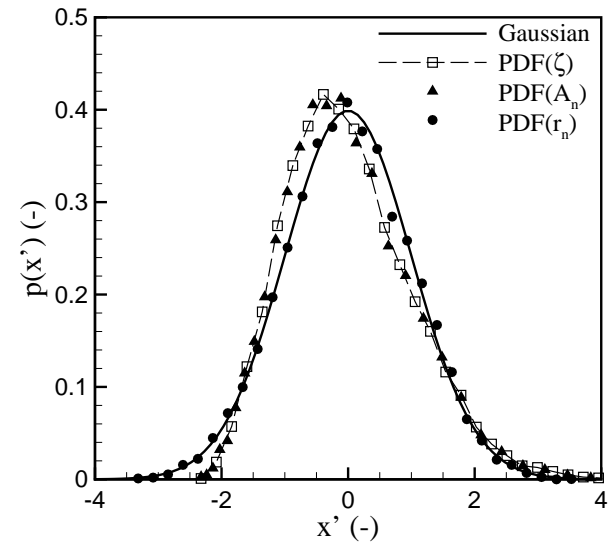

(b)

FiguRE 6.8: Standardized PDFs of $\zeta$ (see Eq. (6.40)), the droplet radius $r_{n}$, and the droplet surface area $A_{n}$ for non-evaporated droplets. For comparison, a Gaussian distribution with zero mean and unity variance is plotted as well. The results have been obtained with the simplified condensation model for sampling time $t_{e}=100 \mathrm{~s}$, and the sampling area $L^{2}=(500 \mathrm{~m})^{2}$. (a) The final altitude of droplets $z_{e}=1350 \mathrm{~m}$. (b) The final altitude of droplets $z_{e}=1380 \mathrm{~m}$.

where $w$ is the vertical velocity component of the flow field, and due to symmetry, it is clear that $\langle\zeta\rangle=\left(z_{e}-z_{\text {ref }}\right) t$. The variance $\left\langle\zeta^{2}\right\rangle-\langle\zeta\rangle^{2}$ then follows from:

$$
\left\langle\zeta^{2}\right\rangle-\langle\zeta\rangle^{2}=2\left\langle w^{2}\right\rangle \int_{0}^{t} \int_{0}^{t^{\prime}} \xi\left(t^{\prime}, t^{\prime \prime} ; t_{e}\right) \mathrm{d} t^{\prime \prime} \mathrm{d} t^{\prime}
$$

where:

$$
\xi\left(t^{\prime}, t^{\prime \prime} ; t_{e}\right)=\int_{t^{\prime}}^{t_{e}} \int_{t^{\prime \prime}}^{t_{e}} R(|\alpha-\beta|) \mathrm{d} \alpha \mathrm{d} \beta .
$$

Here, $R(|x|)$ is the Lagrangian correlation coefficient for which: $R(0)=1$, and:

$$
\int_{0}^{\infty} R(x) d x=\tau_{w}
$$

where $\tau_{w}$ denotes the integral time scale. Due to statistical stationarity of the flow field, the term $\left\langle w^{2}\right\rangle$ is a constant. Differentiation of Eq. (6.45) with respect to time 
then yields:

$$
\frac{\mathrm{d}}{\mathrm{d} t}\left(\left\langle\zeta^{2}\right\rangle-\langle\zeta\rangle^{2}\right)=2\left\langle w^{2}\right\rangle \int_{0}^{t} \xi\left(t, t^{\prime \prime} ; t_{e}\right) \mathrm{d} t^{\prime \prime} .
$$

Since: $R(x)>0$, it follows that: $\xi\left(t, t^{\prime \prime} ; t_{e}\right)>0$, and therefore: $\frac{d}{d t}\left(\left\langle\zeta^{2}\right\rangle-\langle\zeta\rangle^{2}\right)>0$, which implies that the variance of $\zeta(t)$ increases with time. The mean and the variance of the droplet surface distribution $\operatorname{PDF}\left(A_{n}\right)$ at altitude $z_{e}$ can be estimated in the course of time as:

$$
\begin{array}{r}
\left\langle A_{n}\right\rangle=4 \pi r_{0}^{2}+G A\langle\zeta\rangle(t)=4 \pi r_{0}^{2}+G A\left(z_{e}-z_{r e f}\right) t, \\
\frac{\mathrm{d}}{\mathrm{d} t}\left(\operatorname{var}\left(A_{n}\right)\right)=2 G^{2} A^{2}\left\langle w^{2}\right\rangle \int_{0}^{t} \xi\left(t, t^{\prime \prime} ; t_{e}\right) \mathrm{d} t^{\prime \prime},
\end{array}
$$

with $\mathrm{d} / \mathrm{d} t(\operatorname{var}(\zeta))$ given by Eq. (6.48). This shows that the average droplet size increases in size when $z_{e}>z_{\text {ref }}$, and that the PDF of $A_{n}$ becomes broader in the course of time. It is noted that this analysis is only valid when droplet evaporation can be neglected.

Finally, the sensitivity of the droplet size distribution with respect to the sample area size $L$ is investigated. For this purpose the droplet distributions in sampling areas of five different sizes $L^{2}$ are calculated in one realization of the flow field, and the results are shown in Figure 6.9. It appears that the droplet size distribution is broad in all sampling areas, also within sampling areas of the size of $L^{2}=(1 \mathrm{~cm})^{2}$. It is thus demonstrated that the condensation process leads to a broad size distribution within volumes comparable to the smallest scales of turbulence.

Like Celani et al. [17] explain, both large scales and small scales are responsible for the spectral broadening at small scales: large scales are necessary to create sufficiently large differences in supersaturation among droplets, whereas the small-scale fluctuations can mix droplets of different sizes. This process can be illustrated in the current model by calculating the droplet size distribution in a small sampling area of size $L^{2}=(1 \mathrm{~cm})^{2}$ in a flow field in which only the large-scale wavemodes $1 \leq n \leq 10$ are accounted for in Eq. (6.3), and in a simulation in which only the small-scale wavemodes $191 \leq n \leq 200$ are considered. In Figure 6.10.a both results are shown, along with the predictions in which all 200 wave modes have been taken into account. Apparently, neither the model in which the small scales have been neglected, nor the simulation in which the large scales have been neglected are able to predict a broad size distribution in a small sampling space. Furthermore, Figure 6.10.b shows that the distribution is nearly uniform in case only the large scales of motion are included, whereas the distribution tends to be Gaussian-like when only the smallest scales are considered. Since both size distributions are very narrow, it is clear that a realistic model for droplet condensation in clouds should include both the large scales and the 


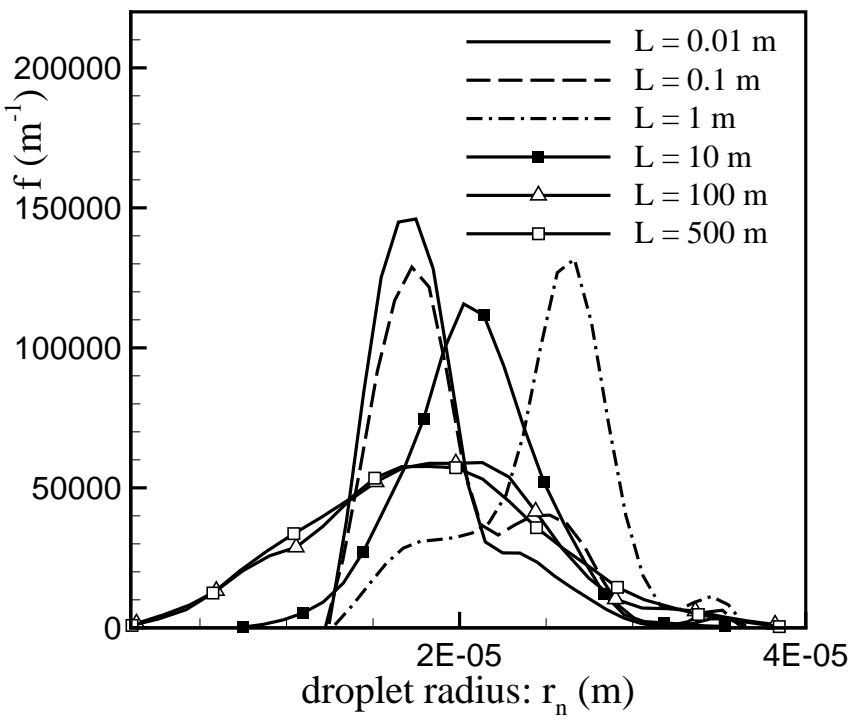

FIGURE 6.9: Droplet radius distribution function $f\left(r_{n}\right)$ as a function of the droplet radius $r_{n}$, for six different sampling areas $L^{2}$ in one realization of the flow field. The sampling time $t_{e}=100 \mathrm{~s}$, and the final altitude of droplets $z_{e}=1350 \mathrm{~m}$. The results have been obtained with the simplified condensation model.

small scales of turbulence, as both of them are essential in the process of spectral broadening.

\subsubsection{Results for two-way coupled condensation model}

All results in the preceding section have been obtained with the simplified condensation model, which does not take into account the feed-back of the droplet condensation on the carrier flow. In this section, the extent to which these phenomena affect the growth of droplets, is quantified. For the two-way coupled condensation model, the initial conditions for the droplet radius, the temperature, and the supersaturation are the same as for the simplified condensation model, and the same holds for the numerical methods used. In the two-way coupled model, two additional parameters have to be chosen that are not needed for the simplified model: the partial air density, which has only minor variations in clouds, is assigned a constant value of $\rho_{a}=1.2 \mathrm{~kg} \mathrm{~m}^{-3}$ in all simulations, whereas the droplet number density $N_{l}$, which may differ from cloud to cloud, is varied.

First, the evolution of the droplet size distribution is shown in Figures 6.11.a and $\mathrm{b}$ for an altitude of $z_{e}=1350 \mathrm{~m}$, and in Figures 6.11.c and $\mathrm{d}$ for an altitude of $z_{e}=1380 \mathrm{~m}$. The sampling area is taken as $L^{2}=(500 \mathrm{~m})^{2}$, whereas the droplet num- 


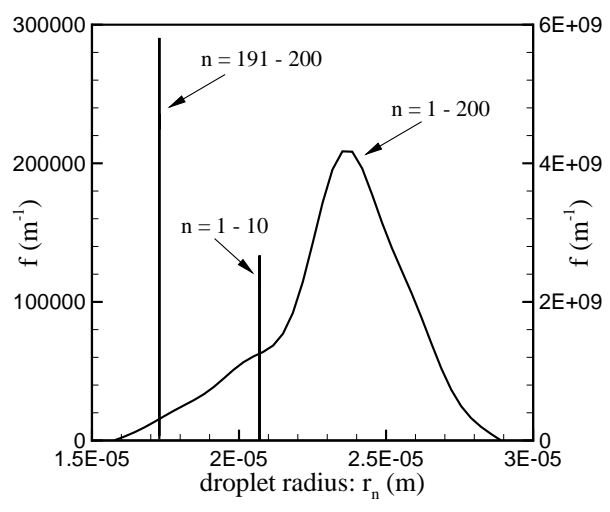

(a)

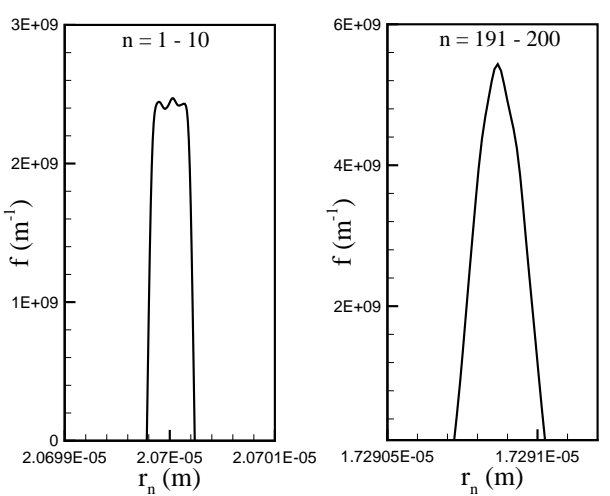

(b)

FIGURE 6.10: Droplet radius distribution function $f\left(r_{n}\right)$ for three different flow fields: one with only the largest scales taken into account $(1 \leq n \leq 10$, correspondingly: $\left.1,26 \cdot 10^{-2} \leq k \leq 2,74 \cdot 10^{-2} \mathrm{~m}^{-1}\right)$, one with only the smallest scales taken into account $\left(191 \leq n \leq 200\right.$, correspondingly: $\left.3,47 \cdot 10^{3} \leq k \leq 6,28 \cdot 10^{3} \mathrm{~m}^{-1}\right)$, and one with all scales taken into account $(1 \leq n \leq 200)$. (a) shows all three results in one figure, where the droplet size distribution function obtained with the full spectrum $(1 \leq n \leq 200)$ should be read from the left scale, the other two from the right scale; (b) shows close-ups of the droplet size distribution function for the large and small scales. The sampling time $t_{e}=100 \mathrm{~s}$, the final altitude of droplets $z_{e}=1350 \mathrm{~m}$ and the sampling area $L^{2}=(0.01 \mathrm{~m})^{2}$. The results have been obtained with the simplified condensation model.

ber density is chosen as $N_{l}=\left(5 \eta_{k}\right)^{-3}$. The data obtained with the simplified coupled model (using the same parameter settings) are also shown (indicated by the lines with filled symbols, also see Figure 6.4) for comparison. From these figures it is clearly observed that vapor depletion and latent heat slow down the droplet growth considerably, so that the mean droplet size predicted with the two-way coupled model is consistently smaller than that predicted with the simplified coupled model. The differences between the two models are especially manifest for $t_{e}>10 \mathrm{~s}$, as the variance of the droplet size distribution for the two-way coupled model is significantly smaller. The initial growth of droplets from sizes of $0.1 \mu \mathrm{m}$ to $5 \mu \mathrm{m}$ does not seem to be significantly affected by vapor depletion and latent heat.

The results plotted in Figure 6.11.c and $\mathrm{d}$ for the higher altitude $z_{e}=1380 \mathrm{~m}$ show the same trends as those corresponding with $z_{e}=1350 \mathrm{~m}$. For both altitudes, the droplet size distributions tend to a steady state, as the results corresponding with $t_{e}=80 \mathrm{~s}$ and $t_{e}=100 \mathrm{~s}$ are almost identical. As can be expected from the analysis presented in the previous section, the mean droplet radius is higher for $z_{e}=1380 \mathrm{~m}$ than for $z_{e}=1350 \mathrm{~m}$. The size distribution predicted with the two-way coupled model 


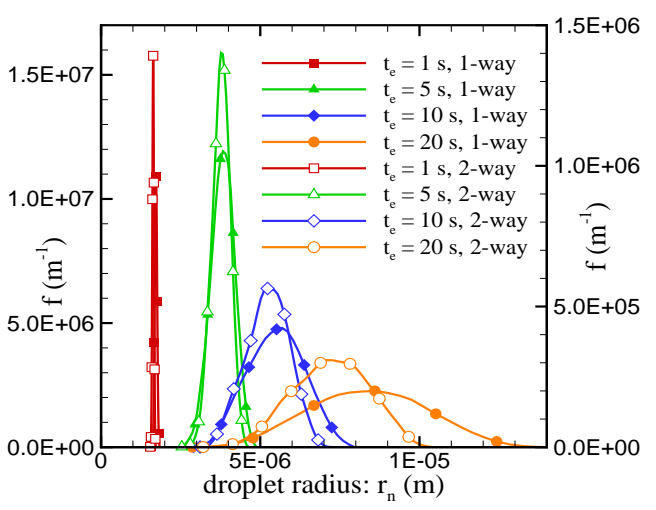

(a)

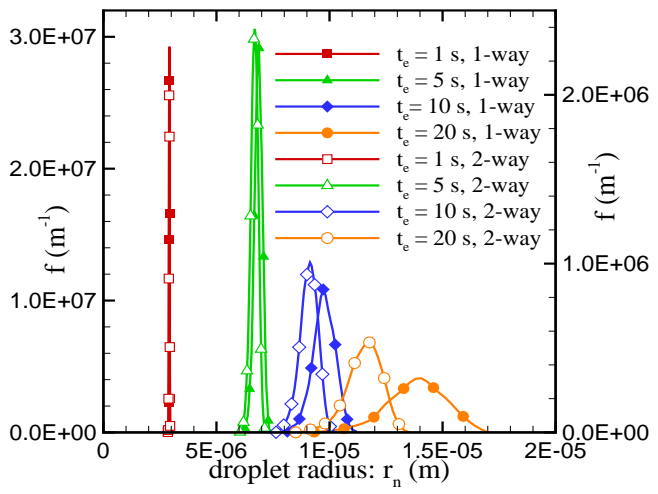

(c)

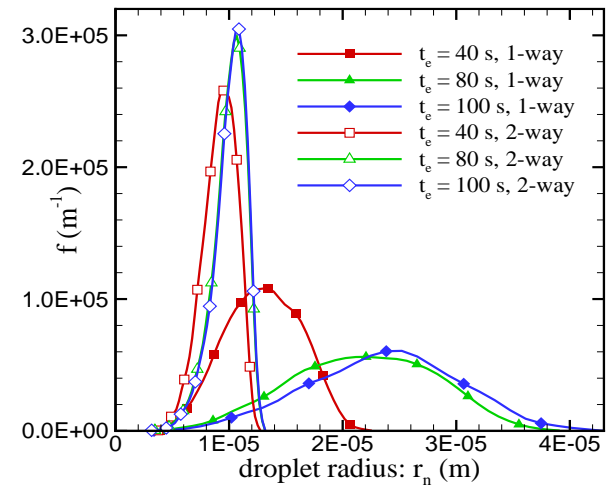

(b)

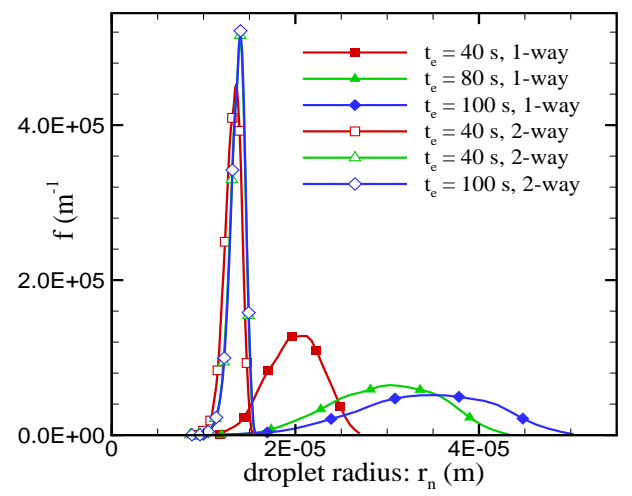

(d)

FIGURE 6.11: Droplet radius distribution function $f\left(r_{n}\right)$ for seven different instants of time $t_{e}:(a)$ and (b) correspond with $z_{e}=1350 \mathrm{~m},(c)$ and $(d)$ correspond with $z_{e}=1380 \mathrm{~m}$. In (a) and (c), the size distributions for $t_{e} \geq 5 \mathrm{~s}$ are plotted on the right vertical scale. The lines with open symbols have been obtained with the two-way coupled condensation model, with $L^{2}=(500 \mathrm{~m})^{2}$ and $N_{l}=\left(5 \eta_{k}\right)^{-3}=0.8 \times 10^{7} \mathrm{~m}^{-3}$. The data for the one-way coupled simulation obtained with the same parameter settings (filled symbols, also see Figure 6.4) are shown for comparison.

is still broad for both altitudes, so it can be concluded that the phenomena of latent heat release and vapor depletion do not qualitatively affect the spectral broadening. They mainly seem to affect the average size and variance of the droplet size distribution, but not the shape of the distribution function itself.

These results can be explained in more detail as follows. If the supersaturation is 
positive, but small $(0<s \ll 1)$, Eq. (6.26) can be rewritten using Eq. (6.31),

$$
\frac{\mathrm{d} s}{\mathrm{~d} t} \simeq \Gamma \mathbf{u} \cdot \mathbf{e}_{z} \frac{1}{\rho_{v}^{s}} \frac{d \rho_{v}^{s}}{d T}-s\left(\frac{1}{\tau_{s}}+\frac{1}{\tau_{L}}\right),
$$

where the time scale $\tau_{L}$ associated with the latent heat release is equal to:

$$
\tau_{L}=\frac{\rho_{a} C_{p, a}}{4 \pi r_{n} N_{l} D_{v, a} L_{b}}\left(\frac{\mathrm{d} \rho_{v}^{s}}{\mathrm{~d} T}\right)^{-1} .
$$

Thus, vapor depletion and latent heat release have very similar effects on the supersaturation. Comparison between Eq. (6.27) and Eq. (6.51) shows that $\tau_{s}$ and $\tau_{L}$ are related by the expression:

$$
\frac{\tau_{s}}{\tau_{L}}=\frac{L_{b}}{\rho_{a} C_{p, a}} \frac{\mathrm{d} \rho_{v}^{s}}{\mathrm{~d} T},
$$

which ratio is plotted in Figure 6.12.a as a function of the temperature surrounding the droplet. Apparently, the effect of latent heat is dominant if $T \gtrsim 280 \mathrm{~K}$, whereas the effect of vapor depletion is more important if $T \lesssim 280 \mathrm{~K}$.

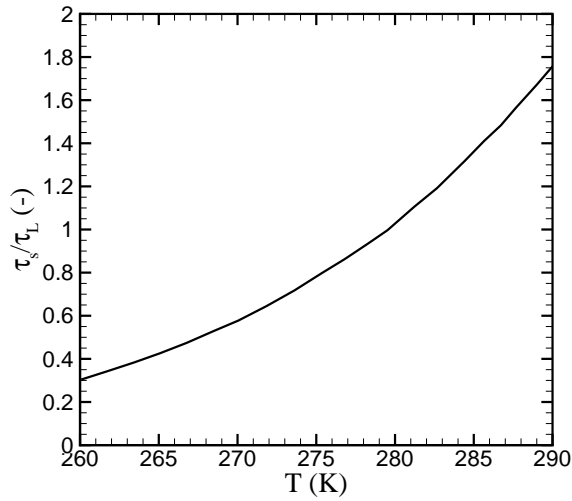

(a)

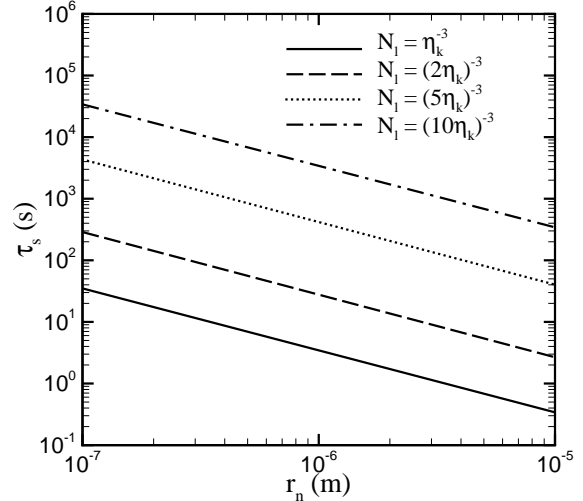

(b)

FIGURE 6.12: (a) Ratio between the vapor depletion time scale and the latent heat time scale $\tau_{L}$, as a function of the temperature $T$. The partial air density is taken as $\rho_{v}=1.2 \mathrm{kgm}^{-3}$ and the pressure is $10^{5} \mathrm{~Pa}$. (b) Vapor depletion time scale $\tau_{s}$ as a function of the droplet radius, for four different values of the droplet number density $N_{l}$. The temperature is taken as $T=280 \mathrm{~K}$ and the pressure as $10^{5} \mathrm{~Pa}$.

The actual value of $\tau_{s}$ depends only weakly on the pressure and the temperature through $D_{v, a}$. Using Eq. (6.27), $\tau_{s}$ has been calculated for several number densities $N_{l}$ and droplet radii $r_{n}$; the result is plotted in Figure 6.12.b. 
The time scale $\tau_{s}$ is inversely proportional to $r_{n}$, and hence the effect of vapor depletion (and thus also the effects of latent heat release at a given temperature, see Figure 6.12.a ) becomes more important as the droplet grows. For instance, at a number density $N_{l}=\left(5 \eta_{k}\right)^{-3}$ and a droplet radius smaller than $5 \mu m, \tau_{s}$ is much larger than the time scale in which the condensation process takes place $\left(L_{0} / U_{0} \sim 100 s\right)$. Therefore, the condensation process of droplets with $r_{n} \lesssim 5 \mu \mathrm{m}$ is hardly affected by vapor depletion and latent heat release, which is confirmed by Figures 6.11.a-d.

Figure 6.12.b also illustrates the importance of the droplet number density: the higher the number density, the less volume of air/water mixture is available per droplet and the less the droplets are able to grow. This is confirmed by Figure 6.13, where the droplet distribution function at $z_{e}=1350 \mathrm{~m}$ after $t_{e}=100 \mathrm{~s}$ has been plotted. Indeed, the droplet growth is most impeded for the highest number density considered $\left(N_{l}=\eta_{k}^{-3}\right)$, whereas the graph for $N_{l}=\left(10 \eta_{k}\right)^{-3}$ is only slightly different from the droplet size distribution at $t_{e}=100 \mathrm{~s}$ shown in Figure 6.4.

Finally, the sensitivity of the resulting droplet size distributions to the size of the sampling area $L$ is examined. The droplet size distributions obtained for the two-way coupled model with $N_{l}=\left(2 \eta_{k}\right)^{-3}$ are plotted in Figure 6.14 for six different values of $L$ for one realization of the flow field. A broad droplet size distribution is observed for $L \geq 10 \mathrm{~m}$, whereas the variance is much smaller for $L \leq 1 \mathrm{~m}$. This is different from the results obtained with the simplified model (see Figure 6.9), for which the variance was observed to be almost equal for all sampling sizes. This difference in behavior between the two-way coupled and the simplified model can be explained when one compares the typical time scale for dispersive motion of droplets to set in (denoted by $\tau_{d}$ ) to the condensation time scale $\tau_{s}$. For droplets which are released in a small sampling space and traced backward in time, dispersive motion sets in much later compared to droplets which are released in larger sampling spaces, meaning that for the latter $\tau_{d}$ is smaller. As all droplets end up at the same altitude $z_{e}$ at time $t=t_{e}$, the dispersive motion takes place during $0<t<t_{e}-\tau_{d}$, whereas the influence of vapor depletion effectively sets in for $t>\tau_{s}$. If $t_{e}-\tau_{d}<\tau_{s}$, the variation in droplet size stems from the dispersive motion through different supersaturation fields. If $t_{e}-\tau_{d}>\tau_{s}$, however, the effect of vapor depletion causes a locally equilibrated size of each droplet regardless of the droplet's origin, which results in a narrower droplet size distribution. This explains why the droplet size distribution is much broader for the larger sampling spaces. Despite this difference in broadening of the droplet size distribution, it may still be concluded that limited spectral broadening does take place, even when the effects of latent heat release and vapor depletion are taken into account (see Figure 6.9).

From the results presented in this section it becomes clear that the droplet size distribution within a fixed sampling area becomes statistically stationary after a sufficiently long time. An estimation of the average droplet size $\left(\left\langle r_{n}\right\rangle_{e q}\right)$ in this equilib- 


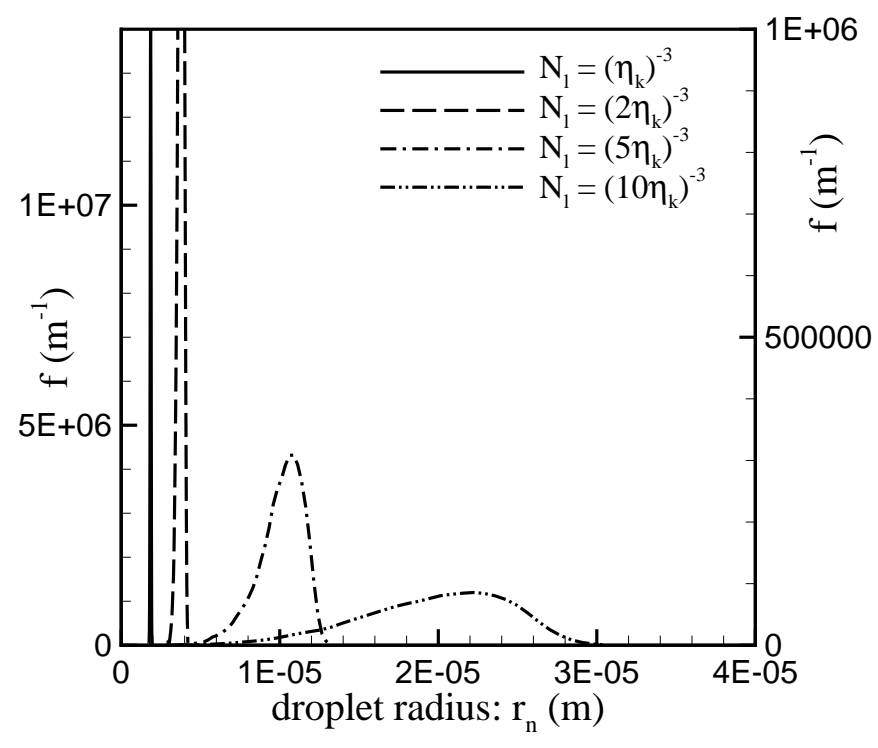

FIGURE 6.13: Droplet radius distribution function $f\left(r_{n}\right)$ at an altitude $z_{e}=1350 \mathrm{~m}$ after a time $t_{e} 100 \mathrm{~s}$, for four different droplet number densities. The results have been obtained with the two-way coupled condensation model, with $L^{2}=(500 m)^{2}$.

rium situation can be made by the following heuristic approach. First, Eq. (6.50) is reformulated in an Eulerian frame of reference as:

$$
\frac{\partial s}{\partial t}+\mathbf{u} \cdot \nabla s=\Gamma \mathbf{u} \cdot \mathbf{e}_{z} \frac{1}{\rho_{v}^{s}} \frac{d \rho_{v}^{s}}{d T}-s\left(\frac{1}{\tau_{s}}+\frac{1}{\tau_{L}}\right) .
$$

To facilitate further analysis, it is assumed that $\|\nabla s\| \ll \Gamma \mathrm{d}\left(\ln \rho_{v}^{s}\right) / \mathrm{d} T$, so that this term can be excluded from Eq. (6.53). By averaging over all droplets in the sampling area, bearing in mind that both $\tau_{s}$ and $\tau_{L}$ depend on the droplet size (see Eqs. (6.27) and (6.51)), and assuming that the variation in $s$ and $T$ over the area is much smaller than the corresponding average values, the following expression is obtained:

$$
\frac{\partial s}{\partial t}=\Gamma \mathbf{u} \cdot \mathbf{e}_{z} \frac{1}{\rho_{v}^{s}} \frac{d \rho_{v}^{s}}{d T}-s\left(\left\langle\frac{1}{\tau_{s}}\right\rangle+\left\langle\frac{1}{\tau_{L}}\right\rangle\right)
$$

If the turbulent velocity fluctuations are accurately described by a Gaussian random noise, then Eq. (6.53) can be written in the form of a stochastic differential equation (SDE) [51]:

$$
\mathrm{d} s \simeq-s\left(\left\langle\frac{1}{\tau_{s}}\right\rangle+\left\langle\frac{1}{\tau_{L}}\right\rangle\right) \mathrm{d} t+\frac{\Gamma}{\rho_{v}^{s}} \frac{d \rho_{v}^{s}}{d T} \sqrt{2 D_{z}} \mathrm{~d} W
$$




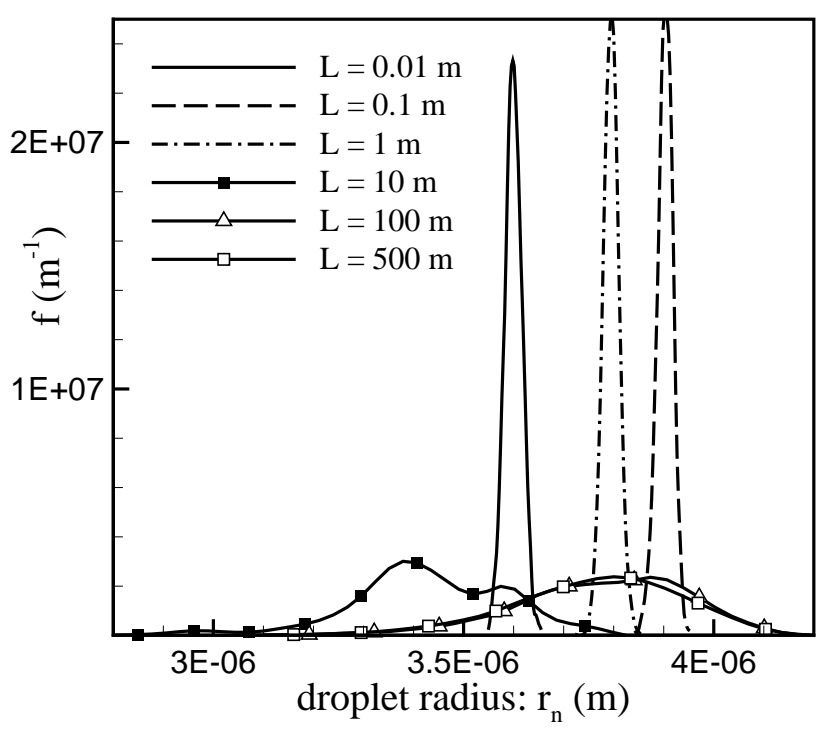

FIGURE 6.14: Droplet radius distribution function $f\left(r_{n}\right)$ at an altitude $z_{e}=1350 \mathrm{~m}$ after a time $t_{e}=100 \mathrm{~s}$, for six different sizes of the sampling area $L^{2}$. The results have been obtained with the two-way coupled condensation model, with $N_{l}=\left(2 \eta_{k}\right)^{-3}=$ $1.25 \times 10^{8} \mathrm{~m}^{3}$.

where $\mathrm{d} W$ denotes an increment of a Wiener process, and $D_{z} \equiv \lim _{t \rightarrow \infty} t^{-1} \frac{1}{2}\left\langle|z(t)-z(0)|^{2}\right\rangle$ is the diffusion coefficient of droplets in the vertical direction. Since the flow is statistically isotropic, $D_{z}=\frac{1}{3} D_{\text {turb }}$, where $D_{\text {turb }}$ is the three-dimensional diffusion coefficient which can be determined from Figure 6.2.a. Eq. (6.55) describes an OrnsteinUhlenbeck process [51], for which the steady-state solution of the PDF is a Gaussian distribution with a mean $\bar{s}=0$ and a variance equal to:

$$
\operatorname{var}(s)=\overline{s^{2}}=\left(\frac{\Gamma}{\rho_{v}^{s}} \frac{d \rho_{v}^{s}}{d T}\right)^{2} D_{z}\left(\left\langle\frac{1}{\tau_{s}}\right\rangle+\left\langle\frac{1}{\tau_{L}}\right\rangle\right)^{-1}
$$

It is noted from Eq. (6.27) and Eq. (6.51) that $\tau_{L}$ and $\tau_{s}$ are both inversely proportional to the droplet radius, so that $\left\langle\frac{1}{\tau_{s}}\right\rangle+\left\langle\frac{1}{\tau_{L}}\right\rangle=\beta\left\langle r_{n}\right\rangle_{e q}$, where $\left\langle r_{n}\right\rangle_{e q}$ is the droplet radius averaged over all droplets situated in the small volume around $\mathbf{x}$, and where the factor $\beta$ is given by:

$$
\beta=4 \pi D_{v, a} N_{l}\left(1+\frac{d\left(\rho_{v}^{S}\right) / d T}{\rho_{a} C_{p a}}\right)
$$


Therefore, Eq. (6.56) can be rewritten as:

$$
\left\langle r_{n}\right\rangle_{e q}=\left(\overline{s^{2}}\right)^{-1} \frac{\left(\frac{\Gamma}{\rho_{v}^{s}} \frac{d \rho_{v}^{s}}{d T}\right)^{2}}{4 \pi N_{l}} \frac{D_{z}}{D_{v, a}}\left(1+\frac{\frac{d \rho_{v}^{s}}{d T}}{\rho_{a} C_{p a}}\right)^{-1} .
$$

Thus, an explicit expression has been obtained for the average droplet radius in an air parcel located at position $\mathbf{x}$, which is valid at statistical equilibrium. Eq. (6.58) depends only on the droplet number density $N_{l}$, the turbulent diffusion coefficient $D_{z}$, the mean-square supersaturation fluctuations $\overline{s^{2}}$ and the temperature (through $D_{v, a}, \Gamma$, $\left(\rho_{v}^{s}\right)^{-1} \mathrm{~d}\left(\rho_{v}^{s}\right) / \mathrm{d} T$ and $\left.\tau_{s} / \tau_{L}\right)$. In the flow field employed in the present study, the turbulent diffusion coefficient $D_{\text {turb }} \simeq 46 \mathrm{~m}^{2} / \mathrm{s}$, as can be deduced from the one-particle statistics in Figure 6.2.a. Therefore, $D_{z}$ is estimated to be $D_{z} \simeq 15 \mathrm{~m}^{2} / \mathrm{s}$. Furthermore, the r.m.s. supersaturation fluctuations are approximately 1\%. Using these values, $\left\langle r_{n}\right\rangle_{e q}$ have been calculated as a function of temperature for four different values of $N_{l}$, and the results are plotted in Figure 6.15.

It is observed that the order of magnitude of $\left\langle r_{n}\right\rangle_{e q}$ corresponds reasonably well to the order of magnitude observed in Figure 6.11.b and Figure 6.11.d, as indicated by the symbols in Figure 6.15. Since the value of $\left\langle r_{n}\right\rangle_{e q}$ decreases moderately with temperature, it is clear that the eventual droplet radius in Figure 6.11.b (see the curve for $t_{e}=100 \mathrm{~s}$ ) is somewhat larger than in Figure 6.11.a. More generally, the present analysis suggests that the largest droplets are found in the coolest regions of a cloud.

\subsection{Conclusions}

In this chapter the condensation of microdroplets in model systems representing atmospheric clouds has been investigated numerically. Droplets have been followed through a synthetic turbulent flow field composed of 200 random Fourier modes, with wave numbers ranging from the integral scale $\left(O\left(10^{2} m\right)\right.$ ) to the Kolmogorov scale $\left(O\left(10^{-3} \mathrm{~m}\right)\right)$. Two fully Lagrangian droplet growth models have been developed: a two-way coupled model which includes adiabatic cooling, vapor depletion and latent heat, and a simplified model in which the latter two are neglected.

The simulations with the simplified model demonstrate that the droplet size distribution becomes broader in the course of time. At higher altitudes, the mean radius of droplets is larger than at lower altitudes and the number of evaporating droplets decreases. It has been shown analytically that the PDF of the droplet surface $A_{n}$ is related to the turbulent dispersion of droplets, and becomes broader in the course of time. At altitudes where the supersaturation is close to zero, the PDF of $A_{n}$ is positively skewed due to the effect of droplet evaporation, whereas the PDF of the droplet radius is very similar to a Gaussian distribution. 


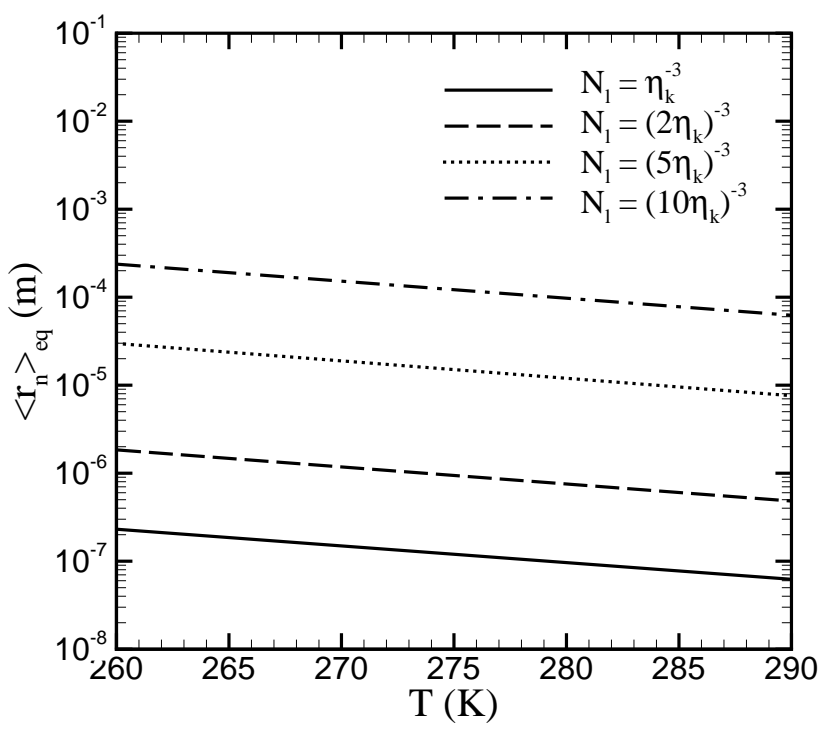

FIGURE 6.15: Long-time value of the average size of droplets in an air parcel $\left\langle r_{n}\right\rangle_{e q}$ as a function of temperature calculated from Eq. (6.58), for four different values of the droplet number density $N_{l}$. The turbulent diffusion coefficient is $D_{z}=15 \mathrm{~m}^{2} / \mathrm{s}$ and the r.m.s. of the supersaturation $\left(\overline{s^{2}}\right)^{1 / 2}=1 \%$. The open circle corresponds with $\left\langle r_{n}\right\rangle_{e q}$ extracted from the data in Figure 6.11.b $\left(z_{e}=1350 \mathrm{~m}, N_{l}=\left(5 \eta_{k}\right)^{-3}\right.$, and $\left.t_{e}=100 \mathrm{~s}\right)$, and the filled circle similarly corresponds with the data in Figure $6.11 . d\left(z_{e}=1380 \mathrm{~m}\right.$, $N_{l}=\left(5 \eta_{k}\right)^{-3}$, and $\left.t_{e}=100 s\right)$.

By testing different ranges of wave modes, it is illustrated that the spectral broadening on centimeter scales is caused by both large scales of turbulence and small scales: large scales transport droplets through regions of different supersaturations, whereas small scales mix droplets of different sizes.

Whilst the simplified condensation model neglects vapor depletion and latent heat release, their effects are slightly overestimated in the two-way coupled condensation model, as mechanisms like diffusion, which allow the parcel to exchange water vapor and thermal energy with its surroundings, have been neglected. Nonetheless the results for the droplet size distributions obtained with the two-way coupled model are qualitatively similar to the results from the simplified model: broad droplet size distributions are found for droplets with radius $r_{n} \simeq 10 \mu \mathrm{m}$. Quantitatively, the droplet sizes obtained are smaller than predicted with the simplified model, and the droplet size distribution in the two-way coupled case reaches a more narrow equilibrium shape after a sufficiently long time. The results have been explained by determining typical time scales for the vapor depletion $\tau_{s}$ and for the latent heat release $\tau_{L}$. It is 
shown that $\tau_{L}$ is smaller than $\tau_{s}$ for high temperatures $T \gtrsim 280 \mathrm{~K}$, so that latent heat release is dominant over vapor depletion in this regime. Vice versa, vapor depletion is more important than latent heat for $T \lesssim 280 \mathrm{~K}$. Since $\tau_{s}$ and $\tau_{L}$ are inversely proportional to the droplet radius $r_{n}$, both effects become more important as the droplet radius grows. This is the reason why an equilibrium is reached when the droplets have become sufficiently large. The average droplet size in the equilibrium situation is of the order of $10 \mu \mathrm{m}$ for a realistic value of the droplet number density $N_{l}$ such as $\left(2 \eta_{k}\right)^{-3}[125]$.

To conclude, the present results demonstrate that the condensation process in a synthetic turbulent flow field leads to a broad droplet size distribution, even within volumes comparable to the smallest scales of the turbulent flow. Although the physics in the model have been significantly simplified, this result does offer a possible explanation for the broadening of the droplet size distribution in actual atmospheric clouds.

Although the approach in this study is tailored to droplet condensation in atmospheric clouds, it could be of importance in other turbulent flows with condensation as well, especially in case the condensation takes place non-homogeneously and in case the time scales of the condensation process are comparable to the time scales of turbulent mixing. 


\section{CONCLUSIONS AND PERSPECTIVE}

This chapter summarizes the main conclusions derived from the investigations in this work. The main challenges related to condensation modelling are briefly reviewed, and suggestions are given for future research.

\subsection{Conclusions}

The thermodynamic model and transport equations for a two-phase liquid/vapor mixture in transonic condensing flow have been presented in chapter 2. The models are specifically tailored to mixtures where the droplet number densities are sufficiently large to employ a continuum modelling approach. Both non-isothermal and isothermal unary condensation models have been discussed, in which the complexities associated with the former have been exposed. Furthermore, the thermodynamics and governing transport equations for systems displaying multi-component condensation have been derived for the case of isothermal condensation.

In chapter 3 the kinetic equation (KE), and its first- and second-order approximations, the general dynamic equation (GDE) and the Fokker-Planck equation (FPE), respectively, have been evaluated, based on (a) a numerical simulation of a nucleation pulse experiment, and (b) an expanding nozzle flow. The former is an illustrative case of quasi-steady nucleation, for which good agreement is found between the size distributions predicted using the various models. In the second test case, the differences between the obtained results are more pronounced, both in terms of the thermodynamic variables and in the predicted droplet size distributions. This was shown to be a consequence of the deviation of the droplet size distribution from its quasi-steady state solution. The solutions of the KE and GDE have been validated by comparing the predicted solutions to measurement data obtained from literature. For the KE specifically, a sensitivity analysis has been carried out in order to explore the extent to which key physical uncertainties affect the predicted solutions. The strongest influence pertains to the equilibrium size distribution, with the semi-empirical Wölk and Strey-corrected Courtney distribution giving the best agreement with reported mea- 
surements. Given this result, the incorporation of empirical data in the condensation models constitutes a potential improvement compared to existing purely theoretical models. For each of the methods used to simulate the nozzle flow, it has been consistently found that the liquid production rate and the corresponding temperature rise are overestimated. Apparently, this is a consequence of using an isothermal condensation model, for which the droplet temperature during condensation is underestimated.

In chapter 4 the method of moments (MOM) has been evaluated, in which two variants of the moment equations (ME) are considered: the ME derived from the kinetic equation (KE), and those derived from the general dynamic equation (GDE). Two closure methods for the MOM, viz. closure based on mean droplet size-approximations, and closure based on quadrature approximations (QMOM and DQMOM), have been qualitatively evaluated, based on key requirements that have been identified from steady state and transient characteristics of the KE. From this it is concluded that neither of the closure methods performs satisfactorily, and that the quadrature approximations suffer severely from a lack of robustness. From a simulation of a condensing nozzle flow, the influence of the closure error in the MOM is quantified. For the particular test case considered, the agreement between results predicted with Hill's MOM and detailed calculations with the KE appears to be reasonable, both for the flow field variables as well as the droplet size distributions. These results suggest that the MOM offers an acceptable trade-off between accuracy and computational expense, which is especially relevant for engineering applications.

In chapter 5 a condensing flow model has been presented, which utilizes a kinetic equation to predict the evolution of a two-component (binary) dispersion, generated by homogeneous nucleation. The model has been implemented in a numerical method which has been verified by means of two test cases reported in the literature. Subsequently, the model has been validated using measurement data from a nozzle flow experiment. Comparison of predicted and measured condensation-onset data has shown a good agreement between theory and experiment. Furthermore, it has been verified that the binary droplet size distribution does not approach its quasisteady state solution as is usually assumed in condensation experiments. Similarly to the case of unary condensation, it is found that the equilibrium size distribution has a profound influence on the predicted results.

Finally, in Chapter 6 an excursion is made into the field of turbulent condensing flow, for a model-system reminiscent of atmospheric clouds. Droplets have been followed through a synthetic turbulent flow field composed of random Fourier modes, with wave numbers ranging from the integral scales to the Kolmogorov scales. Two fully Lagrangian droplet growth models have been proposed: (a) a one-way coupled model in which only adiabatic cooling of a rising air parcel is considered, and (b) a two-way coupled model which also accounts for the effects of local vapor depletion and latent heat release. The simulations with both models have shown that the droplet 
size distribution becomes broader in the course of time, even though droplet growth is stabilized by vapor depletion and latent heat release in the two-way coupled model.

\subsection{Perspective}

Although condensation modelling started early in the twentieth century, there still remains a number of challenges that needs to be addressed, both in theoretical modelling, as well as in numerical simulation. The following list, which attempts to give an overview of specific issues which remain unanswered, is by no means complete:

1. With respect to theoretical modelling there is first and foremost the uncertainty associated with the physics of small droplets (clusters), which are presently still treated by means of macroscopic theory. With the increase in computational power, a number of detailed molecular dynamics simulations (MDS) have been carried out for the condensation of water vapor (e.g. in [145]), but these results have not (yet) been used to derive more complete models for the condensation kinetics and thermodynamics at the microscopic level. Of particular importance is the Gibbs free energy $\Delta G_{n}$ of droplet formation, which has shown to exert a profound influence on both unary and binary condensation processes. In this respect, it would be very instructive to use $\Delta G_{n}$-data predicted with detailed molecular theory, to improve the condensation model that has been employed in the present investigation.

2. In theoretical modelling, there is the issue of properly accounting for nonisothermal effects during homogeneous nucleation. The usual practice in condensation modelling is that a distinction is made between nucleation and droplet growth (e.g. in the GDE or Hill's MOM), with non-isothermal effects being included in the growth-stage only (see e.g. [110]). As the evaporation rate of droplets is strongly dependent on droplet temperature, and because this temperature can be significantly different from the gas/vapor-phase temperature, it is expected that incorporation of non-isothermal effects will significantly alter the predicted nucleation rates. This effect has been considered in a number of previous investigations, but these have either resorted to simplified treatment of the droplet energy balance [141], or they did not include validation with measurement data [7], [10]. The proper way to account for non-isothermal effects is to employ a bi-variate distribution function, which includes both droplet size and temperature as the independent variables (see chapter 2). Such an approach has already been reported in [10], and it is expected that its incorporation into numerical methods, such as the ones that have been used to simulate the test cases of chapter 3, can proceed without significant difficulties. 
3. With respect to multi-component condensation, the uncertainty associated with the compositional structure of small droplets represents a major challenge in condensation modelling. This is especially important for applications involving fluids with limited miscibility. A number of models have been devised to take the effects of surface enrichment into account, but these are not entirely void of unphysical behavior [127]. Recently, the emphasis has shifted towards the use of molecular dynamics simulations to gain more insight into the problem, notably in [21] and [64]. The latter is of special relevance because it attempts to generate a phase-diagram for the composition of aqueous nanodroplets based on MDS-data. By using this data in the condensation model as presented in chapter 5 , it should be possible to obtain reliable predictions of binary condensation for systems in which surface enrichment effects are important.

4. Computationally, the challenge lies in devising reduced models that satisfy the key requirements which have been identified in chapter 4. By way of example, it has been demonstrated that moment methods can yield reasonably accurate predictions for condensing nozzle flows. However, the important issue is whether or not this will still hold for unsteady and three-dimensional flows, such as typically encountered in industrial applications. In this regard it is necessary to devise both robust, consistent and accurate closure methods for application to condensing flow.

5. Finally, there remains the problem of condensation in turbulent flows, which has been addressed in chapter 6 . For the specific case of condensation in atmospheric clouds, a number of improvements can be incorporated in the Lagrangian model of chapter 6. Specifically, the activation (or deactivation) of condensation nuclei can be added, and also the turbulent diffusion of air and vapor at the boundaries of the air/droplet parcels can be simulated. There is, however, a limit to the realism of the physical behavior simulated by the present model, as it utilizes a synthetic turbulent flow field. Nevertheless, such a synthetic field could also be used to perform a preliminary investigation of condensation in systems more akin to rapidly expanding flows. This would be an important step in gaining more insight into the role of turbulence in such condensing flows, and could possibly result in improvements of the Reynolds Averaged Navier-Stokes methods that are currently employed for flows with condensation [108]. 


\section{REFERENCES}

[1] S. Adam and G.H. Schnerr, "Instabilities and bifurcation of non-equilibrium two-phase flows", J. Fluid Mech. 348, 1 (1997).

[2] G.D. van Albada, B. van Leer, and W.W. Roberts, "A comparative study of computational methods in cosmic gas dynamics", Astron. Astrophys. 108, 76 (1982).

[3] V. Alopaeus, M. Laakkonen and J. Aittamaa, "Numerical solution of momenttransformed population balance equation with fixed quadrature points", Chem. Engng. Sci. 61, 4919-4929 (2006).

[4] A. Aly and F. Nicolleau, "Dispersion of heavy particle sets in isotropic turbulence using kinematic simulation", Phys. Rev. E 78, 1 (2008).

[5] A.R. Avetissian, G.A. Phillipov and L.I. Zaichik, "Effects of turbulence and inlet moisture on two-phase spontaneously condensing flows in transonic nozzles", Int. J. heat and Mass Transfer 51, 4195-4203 (2008).

[6] F. Bakhtar and M.T. Mohamaddi Tochai, "An investigation of two-dimensional flows of nucleating and wet steam by the time-marching method", Int. J. Heat Fluid Flow 2 (1980).

[7] J.C. Barrett, C.F. Clement and I.J. Ford, "Energy Fluctuations in homogeneous nucleation theory for aerosols", J. Phys. A.: Math. Gen. 26, 529-548 (1993).

[8] J.C. Barrett and J.S. Jheeta, "Improving the accuracy of the moments method for solving the aerosol general dynamic equation", J. Aerosol. Sci. 27, 1135 (1996).

[9] J.C. Barrett and N.A. Webb, "A comparison of some approximate methods for solving the aerosol general dynamic equation”, J. Aerosol. Sci. 29, 31 (1998).

[10] J.C. Barrett, "A stochastic simulation of nonisothermal nucleation", J. Chem. Phys. 128, 164519 (2008). 
[11] K.V. Beard and H.T.J. Ochs, "Warm-Rain Initiation: An Overview of Microphysical Mechanisms", J. Applied Meteorology, 32, 608-625 (1993).

[12] J. Bec, A. Celani, M. Cencini and S. Musacchio, "Clustering and collisions of heavy particles in random smooth flows", Phys. Fluids 17, 073301 (2005).

[13] R. Becker and W. Döring, "Kinetische behandlung der keimbildung in übersättigten Dämpfern”, Ann. Phys. 416, 719-752 (1935).

[14] R.B. Bird, W.E. Stewart, and E.N. Lightfoot, Transport Phenomena, (John Wiley \& Sons, Inc, New York, 2002).

[15] C.E. Brennen, Fundamentals of Multiphase Flow, (Cambridge University press, Cambridge, 2005).

[16] J.R. Brock and J. Oates, "Moment simulation of aerosol evaporation", J. Aerosol. Sci. 18, 59, (1987).

[17] A. Celani, G. Falkovich, A. Mazzino and A. Seminara, "Droplet condensation in turbulent flows", Europhysics Letters 70, 6, 775-781 (2005).

[18] A. Celani, A. Mazzino, A. Seminara and M. Tizzi, "Droplet condensation in two-dimensional Bolgiano turbulence", Journal of Turbulence 8, 17, 1-9 (2007).

[19] L. Chaumat, J.L. Brenguier, "Droplet Spectra Broadening in Cumulus Clouds. Part I: Broadening in Adiabatic Cores", J. Atm. Sci. 58, 628-641 (2001).

[20] L. Chaumat, J.L. Brenguier, "Droplet Spectra Broadening in Cumulus Clouds. Part II: Microscale Droplet Concentration Heterogeneities", J. Atm. Sci. 58, 642-654 (2001).

[21] B. Chen, I.J. Siepmann and M.L. Klein, "Simulating the Nucleation of Water/Ethanol and Water/n-Nonane Mixtures: Mutual Enhancement and TwoPathway Mechanism", J. Am. Chem. Soc. 125, 3113-3118 (2003).

[22] R. Clift, J.R. Grace, and M.E. Weber, Bubbles, Drops, and Particles, (Academic Press Inc., New York, 1978).

[23] R. Courant, K. Friedrichs, and H. Lewy, "Über die partiellen Differenzengleichungen der mathematischen Physik", Math. Ann., 100, 1, 3274 (1928).

[24] C. T. Crowe, J. N. Chung and T. R. Troutt, Particulate Two-Phase Flow, Heinemann, Oxford (1993).

[25] C.T. Crowe (ed.), Multiphase flow handbook, (CRC Press, New York, 2005). 
[26] C.T. Crowe, M. Sommerfeld, and Y. Tsuji, Multiphase Flow with Droplets and Particles, (CRC Press, Boca Raton, 1998).

[27] C.F. Delale, G.H. Schnerr, and J. Zierep, "The mathematical theory of thermal choking in nozzle flows", Z. angew. Math. Phys. 44, 943-976 (1993).

[28] F. Duan and C.A. Ward, "Surface-thermal capacity of $D_{2} O$ from measurements made during steady-state evaporation", Phys. Rev. E 72, 056304 (2005).

[29] J.R. Fessler, J.D. Kulick and J.K. Eaton, "Preferential concentration of heavy particles in a turbulent channel flow", Physics of Fluids 6(11), 37423749 (1994).

[30] S.P. Fisenko and G. Wilemski, "Kinetics of binary nucleation of vapors in size and composition space", Phys. Rev. E 70, 056119 (2004).

[31] R.C. Flagan, "A thermodynamically consistent kinetic framework for binary nucleation", J. Chem. Phys. 113, 17, 7317-7329 (2007).

[32] H. Flood, "Tröpfchenbildung in Öbersättigten ÄthylalkoholWasserdampfgemischen, Z. phys. Chem. A170,286294 (1934).

[33] M. Frenklach, "Method of moments with interpolative closure", Chem. Engng. Sci. 57, 2229 (2002).

[34] S.K. Friedlander, Smoke, Dust and Haze, (John Wiley \& Sons, New York, 1977).

[35] J.C.H. Fung and J.C. Vassilicos, "Two-particle dispersion in turbulentlike flows", Phys. Rev. E 57(2), 1677-1690 (1998).

[36] A.G. Gerber and A. Mousavi, "Application of quadrature method of moments to the polydispersed droplet spectrum in transonic steam flows with primary and secondary nucleation”, Appl. Math. Modelling 31, 1518-1533 (2007).

[37] M. Gharibeh, Y. Kim, U. Dieregsweiler, B.E. Wyslouyzil, D. Ghosh and R. Strey, "Homogeneous nucleation of n-propanol, n-butanol, and n-pentanol in a supersonic nozzle", J. Chem. Phys. 122, 094512 (2005).

[38] R.G. Gordon, "Error Bounds in Equilibrium Statistical Mechanics", J. Math. Phys. 9, 655-663 (1968).

[39] G. Gyarmathy, "The spherical droplet in gaseous carrier streams: review and synthesis", in Multiphase Science and Technology, vol. 1, 99-279 (Springer, Berlin, 1982). 
[40] B.A. van Haarlem, The dynamics of particles and droplets in atmospheric turbulence, $\mathrm{PhD}$ thesis, Delft University of Technology (2000).

[41] R. Hagmeijer, "Equivalence of two different integral representations of droplet distribution moments in condensing flow", Phys. Fluids 16, 176, (2004).

[42] R. Hagmeijer, R.H.A. IJzermans, F. Put, "Solution of the general dynamic equation along approximate fluid trajectories generated by the method of moments", Phys. Fluids 17, 056101 (2005).

[43] J. Halama and J. Forť, "Numerical simulation of 2D and 3D transonic flow of wet steam in turbine cascades", $6^{\text {th }}$ International Conference on Multiphase Flow 2007, Leipzig-Germany, paper no. S3_Fri_B_65 (2007).

[44] C.H. Heath, K.A. Streletzky, B.E. Wyslouzil, J. Wölk and R. Strey, "Small angle neutron scattering from $\mathrm{D}_{2} \mathrm{O}-\mathrm{H}_{2} \mathrm{O}$ nanodroplets and binary nucleation rates in a supersonic nozzle", J. Chem. Phys. 118, 12, 5465-5473 (2003).

[45] P.G. Hill, "Condensation of water vapor during supersonic expansion in nozzles", J. Fluid Mech. 25, 593 (1966).

[46] V. Holten and M.E.H. van Dongen, "Comparison between solutions of the general dynamic equation and the kinetic equation for nucleation and droplet growth", J. Chem. Phys. 130, 1, 014102-1/8 (2009).

[47] M. Ishii, T. Hibiki, Thermo-fluid dynamics of two-phase flow, (Springer, New York, 2006).

[48] R.H.A. IJzermans, Dynamics of dispersed heavy particles in swirling flow, $\mathrm{Ph} . \mathrm{D}$. Thesis, University of Twente (2007).

[49] V. John, I. Angelov, A.A. Öncül and D. Thévenin, 'Techniques for the reconstruction of a distribution from a finite number of its moments", Chem. Engng. Sci. 62, 2890-2904 (2007).

[50] C.H. Jung, S.H. Park and Y.P. Kim, "Size distribution of polydispersed aerosols during condensation in the continuum regime: Aanalytic approach using the lognormal moment method”, J. Aerosol. Sci. 37, 10, 1400-1406 (2006).

[51] N.G. van Kampen, "Stochastic Processes in Chemistry and Physics", Elsevier, Noord-Holland (1992).

[52] D. Kashchiev, Nucleation: basic theory with applications, (ButterworthHeineman, Oxford, 2000). 
[53] P.H. Kelleners, An edge-based finite volume method for inviscid compressible flow with condensation, Ph.D. Thesis, University of Twente (2007).

[54] M. Kielkiewicz, "Accuracy of the moments method", Ann. Nucl. Energy 21, 189, (1994).

[55] R.H. Kraichnan, "Diffusion by a random velocity field", Physics of Fluids 13(1), 22-31 (1970).

[56] M. Kulmala, A. Laaksonen, and S. Gerschick, "The self-consistency correction to homogeneous nucleation: extension to binary systems", J. Aerosol Sci. 23, 3, 309-312 (1992).

[57] A. Laaksonen, R. McGraw, and H. Vehkamäki, "Liquid-drop formalism and free-energy surfaces in binary homogeneous nucleation theory", J. Chem. Phys. 111, 5, 2019-2027 (1999).

[58] G. Lamanna, On nucleation and droplet growth in condensing nozzle flows, $\mathrm{PhD}$ thesis, Eindhoven University of Technology (2000).

[59] C.B. Laney, Computational Gasdynamics, (Cambridge University Press, New York, 1998).

[60] A.S. Lanotte, A. Seminara, and F. Toschi, "Cloud droplet growth by condensation in homogeneous isotropic turbulence", J. Atmosph. Sc. (2008).

[61] K.W. Lee, "Change of particle size distribution during Brownian coagulation", J. Colloid Interface Sci. 92, 315, (1983).

[62] B. van Leer, "Flux-vector splitting for the Euler equations". In Eighth International Conference on Numerical Methods in Fluid Dynamics, Lecture Notes in Physics (1982).

[63] R.J. LeVeque, "Balancing source terms and gradients in high resolution Godunov methods: the quasi-steady wave propagation algorithm", J. Comp. Phys. 146, 346-365 (1998).

[64] J. Li and G. Wilemski, "A structural phase diagram for model aqueous organic nanodroplets", Phys. Chem. Chem. Phys. 8, 1266-1270, (2006).

[65] H.W. Liepmann and A. Roshko, Elements of Gasdynamics, (John Wiley and Sons, New York, 1957).

[66] C.C.M. Luijten, Nucleation and droplet growth at high pressure, Ph.D. Thesis, Eindhoven University of Technology (1998). 
[67] X. Luo, Unsteady flows with phase transition, Ph.D. Thesis, Eindhoven University of Technology (2004).

[68] X. Luo, B. Prast, M.E.H. van Dongen, H.W.M. Hoeijmakers and J. Yang, "On phase transition in compressible flows: modelling and validation", J. Fluid Mech. 548, pp.403-430 (2006).

[69] D.L. Marchisio, J.T. Pikturna, R.O. Fox, R.D. Vigil, and A.A. Barresi "Quadrature Method of Moments for Population-Balance Equations", AIChE Journal 49, 5 (2003).

[70] D.L. Marchisio, R.O. Fox, "Solution of population balance equations using the direct quadrature method of moments", J. Aerosol. Sci. 36, 43 (2005).

[71] F. Maršík, T. Němec, J. Hrubý, P. Demo, Z. Kožíšek, V. Petr, and M. Kolovratník, "Binary Homogeneous Nucleation in Selected Aqueous Vapor Mixtures", J. Solution Chem. 37, 1671-1708 (2008).

[72] M.R. Maxey and J.J. Riley, "Equation of motion for a small rigid sphere in a non-uniform flow", Phys. Fluids 26, 4, pp.883-889 (1983).

[73] M. McCallum and R. Hunt, "The flow of wet steam in a one-dimensional nozzle", Int. J. Numer. Meth. Engng. 44, 1807, (1999).

[74] R. McGraw, "Two-dimensional kinetics of binary nucleation in sulfuric acidwater mixtures", J. Phys. Chem. 102, 5, 2098-2108 (1995).

[75] R. McGraw, "Description of the aerosol dynamics by the quadrature method of moments", Aerosol Sci. Technol. 27, 255 (1997).

[76] R. McGraw, S. Nemesure, and S.E. Schwartz, "Properties and evolution of aerosols with size distributions having identical moments", J. Aerosol. Sci. 29, 761 (1998).

[77] J. Merikanto, E. Zapadinsky, A. Lauri, and H. Vehkamäki, "Origin of the Failure of Classical Nucleation Theory: Incorrect Description of the Smallest Clusters", Phys. Rev. Lett. 98, 145702, (2007).

[78] M. Moheban and J.B. Young, "A study of thermal non-equilibrium effects in low-pressure wet-steam turbines using a blade-to-blade time-marching technique”, Int. J. Heat Fluid Flow 6, 269 (1985).

[79] M. Muitjens, Homogeneous condensation in a vapor/gas mixture at high pressures in an expansion cloud chamber, Ph.D. Thesis, Eindhoven University of Technology (1996). 
[80] W.K. Nicholson, Linear Algebra with Applications, (third edition, PWS Publishing Company, Boston, 1986).

[81] F. Nicolleau, G. Yu, "Two-particle diffusion and locality assumption", Physics of Fluids 16, 7, 2309-2321 (2004).

[82] Y. Okada and Y. Hara, "Calculation of the sticking probability of a water molecule to a water cluster", Earozory Kenkyu (Journal of Aerosol Research, ISSN: 1881-543X), 22, no. 2, p.147-151 (2007).

[83] A. Papoulis, Probability, Random variables and Stochastic Processes, (McGraw-Hill, New York, 1991).

[84] P. Peeters, J.J.H. Gielis and M.E.H. van Dongen, "The nucleation behaviour of supercooled water vapor in helium", J. Chem. Phys. 117, 5647, (2002).

[85] P. Peeters, Nucleation and Condensation in Gas-Vapor Mixtures of Alkanes and Water, Ph.D. Thesis, Eindhoven University of Technology (2002).

[86] B.E. Poling, J.M. Prausnitz, and J.P. O'Connell, The Properties of Gases and Liquids, (fifth edition, McGraw-Hill, Singapore, 2001).

[87] S.B. Pope, Turbulent Flows, Cambridge University Press (2000).

[88] S.E. Pratsinis, "Simultaneous nucleation, condensation and coagulation in aerosol reactors", J. Colloid Interface Sci. 124, 416, (1988).

[89] W.A. Press, S.A. Teukolsky, W.T. Vetterling and B.P. Flannery, Numerical Recipes in Fortran 77, (Cambridge University Press, Cambridge, 1992).

[90] H.R. Pruppacher and J.D. Klett, Microphysics of clouds and precipitation, (D. Reidel Publishing Company, Dordrecht Holland, 1978).

[91] F. Put, Numerical simulation of condensation in transonic flows, Ph.D. Thesis, University of Twente (2003).

[92] D.S. van Putten, Reconstruction of the Multi-Component Droplet Size Distribution in Three-Dimensional Condensing Flow, Master's thesis, Department of Mechanical Engineering, University of Twente (2007).

[93] L. Råde, and B. Westergren, Mathematics Handbook for Science and Engineering, ( $4^{\text {th }}$ edition, Springer-Verlag, New York, 1999).

[94] L.F. Richardson, "Atmospheric diffusion shown on a distance-neighbour graph", Proc. Royal Soc. London. Ser. A., 110(756), 709737 (1926). 
[95] H. Risken, The Fokker-Planck Equation: Methods of Solution and Applications, (Springer, Berlin, 1989).

[96] Z. Rusak and J. Lee, "Transonic flow of moist air around a thin airfoil with nonequilibrium and homogeneous condensation”, J. Fluid Mech. 403, pp.173-199 (2000).

[97] H. Reiss, "The Kinetics of Phase Transitions in Binary Systems", J. Chem. Phys. 18, 6, 840-848 (1950).

[98] U. Sachs, Evaluation of three Condensation Models for Condensing Flow, Master's thesis, Department of Mechanical Engineering, University of Twente (2009).

[99] G.K. Schenter, S.M. Kathmann and B.C. Garrett, "Dynamical benchmarks of the nucleation kinetics of water", J. Chem. Phys. 116, 10, 4275-4280 (2002).

[100] G.H. Schnerr, "2D-transonic flow with energy supply by homogeneous condensation: Onset condition and 2D-structure of steady Laval nozzle flow", Exp. Fluids 7, 145-156 (1989).

[101] J.H. Seinfeld, Atmospheric chemistry and physics of air pollution, (Wiley, New York, 1986).

[102] Y. Seo and J.R. Brock, "Distributions for moment simulation of aerosol evaporation", J. Aerosol. Sci. 21, 511, (1990).

[103] A. Shavitt and Ch. Gutfinger, Thermodynamics. From concepts to applications, (Prentice Hall Europe, Hertfordshire, 1995).

[104] R.A. Shaw, "Particle-turbulence interactions in atmospheric clouds", Ann. Review of Fluid Mechanics 35, 183-227 (2003).

[105] J.A. Shohat and J.D. Tamarkin, The problem of moments, (American Mathematical Society, 1963).

[106] C. Shu, "High order finite difference and finite volume schemes and discontinuous Galerkin methods for CFD”, Int. J. Comp. Fluid Dyn. 17, 2 (2003).

[107] R. Sidin and R. Hagmeijer, "Evaluation of the phase path analysis method in condensing flows", in Proceedings of the $6^{\text {th }}$ International Conference on Multiphase Flow, edited by M. Sommerfeld, paper no. S4_Thu_D_61 (ISBN 9783-86010-913-7, Martin-Luther-Universität Halle-Wittenberg, Halle-Germany, 2007). 
[108] D.A. Simpson and A.J. White, "Viscous and unsteady flow calculations of condensing steam in nozzles", Int. J. Heat and Fluid Flow 26, pp.71-79 (2005).

[109] J.A. Simpson and E.S.C. Weiner, The Oxford English Dictionary, (Clarendon Press, Oxford, 1989).

[110] S. Sinha, B.E. Wyslozuil, and G. Wilemski, "Modeling of $\mathrm{H}_{2} \mathrm{O} / \mathrm{D}_{2} \mathrm{O}$ condensation in supersonic nozzles", Aer. Sci. Techn. 43, 9-24 (2009).

[111] W.A. Sirignano, Fluid Dynamics and Transport of Droplets and Sprays , (first edition, Cambridge University Press, Cambridge, 1999).

[112] S.A. Skillings and R. Jackson, "A robust time-marching solver for onedimensional nucleating steam flows", Int. J. Heat Fluid Flow 8, 139 (1987).

[113] P. D. M. Spelt and A. Biesheuvel,'On the motion of gas bubbles in homogeneous isotropic turbulence", J. Fluid Mech. 336, 221-244 (1997).

[114] D. Stauffer, "Kinetic theory of two-component ("heteromolecular") nucleation and condensation", J. Aerosol Sci. 7, 319-333 (1976).

[115] J. Stoer and R. Bulirsch, Introduction to Numerical Analysis , (second edition, Springer-Verlag, New York, 1963).

[116] G. Strang, "On the construction and comparison of different schemes", SIAM J. Numer. Anal. 5, pp.506-517 (1968).

[117] L.F. Sukhodub, Y.V. Telezhenko, and V.S. Shelkovskii, ”Thermodynamical characteristics of small water clusters", Zhurnal Strukturnoi Khimii 29, 5, pp. 89-94 (1988).

[118] P. Tamamidis and D. Assanis, "Evaluation of various high-order accuracy schemes with and without flux limiters", Int. J. Num. Methods Fluids 16, 931948 (1993).

[119] S. Tanimura, Y. Zvinevich, B.E. Wyslouzil, M.S. Zahniser, J.H. Shorter, D.D. Nelson, and J.B. McManus "Temperature and gas-phase composition measurements in supersonic flows using tunable laser diode absorption spectroscopy: The effect of condensation on the boundary-layer thickness", J. Chem. Phys. 122, 194304 (2005).

[120] S. Tanimura, B.E. Wyslouzil, M.S. Zahniser, J.H. Shorter, D.D. Nelson, and J.B. McManus, "Tunable diode laser absorption spectroscopy study of $\mathrm{CH}_{3} \mathrm{CH}_{2} \mathrm{OD} / \mathrm{D}_{2} \mathrm{O}$ binary condensation in a supersonic Laval nozzle", J. Chem. Phys. 127, 034305 (2007). 
[121] G.I. Taylor, "Diffusion by continuous movements", Ann. Review of Fluid Mechanics 20, 196-212 (1921).

[122] D.J. Thomson, B.J. Devenish, "Particle pair separation in kinematic simulations", J. Fluid Mech. 526, 277-302 (2005).

[123] S. Twomey, "The nuclei of natural cloud formation; Part II:The supersaturation in natural clouds and the variation of cloud droplet concentration", Geofis. Pura Appl. 43, 243-249 (1959).

[124] P.A. Vaillancourt, M.K. Yau, and W.W. Grabowski, "Microscopic Approach to Cloud Droplet Growth by Condensation. Part I: Model Description and Results without Turbulence", J. Atm. Sci. 58, 1945-1964 (2001).

[125] P.A. Vaillancourt, M.K. Yau, P. Bartello, and W.W. Grabowski,'Microscopic Approach to Cloud Droplet Growth by Condensation. Part II: Turbulence, Clustering, and Condensational Growth", J. Atm. Sci. 59, 24, 3421-3435 (2002).

[126] H. Vehkamäki, P. Paatero, M. Kulmala, and A. Laaksonen, "Binary nucleation kinetics: A matrix method", J. Chem. Phys. 101, 11, 9997-10002 (1994).

[127] H. Vehkamäki, Classical Nucleation Theory in Multicomponent Systems, (Springer-Verlag, Berlin, 2006).

[128] P.P. Wegener, J.A. Clumpner, and B.J.C. Wu, "Homogeneous Nucleation and Growth of Ethanol Drops in Supersonic Flow", Phys. Fluids 15, 11 (1972).

[129] W.H. White, "Particle size distributions that cannot be distinguished by their integral moments", J. Colloid Interface Sci. 135, 297 (1990).

[130] A.H. White and J.B. Young, "Time marching method for the prediction of two-dimensional unsteady flows of condensing steam", AIAA J. Propulsion and Power 9, 579 (1993).

[131] A.H. White, "A comparison of modelling methods for polydispersed wetsteam flow", Int. J. Numer. Meth. Engng 57, 819 (2003)

[132] A.J. White and M.J. Hounslow, "Modelling droplet size distributions in polydispersed wet-steam flows", Int. J. Heat Mass Transfer 43, 1873, (2000).

[133] A.H. White and J.B. Young "Transient calculations of nucleation and droplet growth for wet-steam expansions", ICPWS XV, Berlin, September 8-11 (2008).

[134] G. Wilemski, "The Kelvin-equation and self-consistent nucleation theory", J. Chem. Phys. 103, 3, 1119-1126 (1995). 
[135] G. Wilemski and B.E. wyslouzil "Binary nucleation kinetics. I. Self-consistent size distribution", J. Chem. Phys. 103, 3, 1127-1136 (1995).

[136] M. Wilkinson, B. Mehlig, S Östlund and K.P. Duncan, ”Unmixing in random flows", Physics of Fluids 19, 113303 (2007).

[137] M.M.R. Williams, "Some topics in nuclear aerosol dynamics", Prog. Nucl. Energy 17, 1, (1986).

[138] M.M.R. Williams and S.K. Loyalka, Aerosol Science Theory and Practise, (Pergamon Press, Oxford, 1991).

[139] J. Wölk and R. Strey, "Homogeneous nucleation of $\mathrm{H}_{2} \mathrm{O}$ and $\mathrm{D}_{2} \mathrm{O}$ in comparison: the isotope effect", J. Phys. Chem. B 105, 11683-11701 (2001).

[140] D.L. Wright Jr, "Numerical advection of moments of the particle size distribution in Eulerian models", J. Aerosol. Sci. 38, 352-369 (2006).

[141] B.E. Wyslouzil and J.H. Seinfeld, "Nonisothermal homogeneous nucleation", J. Chem. Phys. 97, 4, 2661-2670 (1992).

[142] B.E. Wyslouzil and G. Wilemski, "Binary nucleation kinetics. II. Numerical solution of the birth-death equations", J. Chem. Phys. 103, 3, 1137-1151 (1995).

[143] B.E. Wyslouzil and G. Wilemski, "Binary nucleation kinetics. III. Transient behavior and time lags", J. Chem. Phys. 105, 3, 1090-1100 (1996).

[144] B.E. Wyslouzil, Ch.H. Heath, and J.L. Cheung, "Binary condensation in a supersonic nozzle", J. Chem. Phys. 113, 17, 7317-7329 (2000).

[145] K. Yasuoka and M. Matsumoto, "Molecular dynamics of homogeneous nucleation in the vapor phase. II. Water", J. Chem. Phys. 109, 19, 8463-8470 (1998).

[146] J.B. Young, "The condensation and evaporation of liquid droplets in a pure vapour at arbitrary Knudsen number", Int. J. heat Mass Transfer 34, 7, pp. 16491661 (1992).

[147] J.B. Young, "Two-dimensional, non-equilibrium, wet-steam calculations for nozzles and turbine cascades", J. Turbomachinery 114, 569 (1992).

[148] J.B. Young, "The condensation and evaporation of liquid droplets at arbitrary Knudsen number in the presence of an inert gas", Int. J. heat Mass Transfer 36, 11, pp. 2941-2956 (1993).

[149] J.B. Young, "The fundamental equations of gas-droplet multiphase flow", Int. J. Multiphase Flow 21, 2, pp. 175-191 (1994). 



\section{FLUID PROPERTIES}

\section{Note:}

- All variables are expressed in S.I. units

- Under ambient conditions: $T=298.15 \mathrm{~K}$ and $p=1.013 \cdot 10^{5} \mathrm{Nm}^{-2}$.

- Universal gas constant: $R_{u}=8.314 \mathrm{Jmol}^{-1} K^{-1}$

\section{A.1 Properties of water $\left(\mathrm{H}_{2} \mathrm{O}\right)$}

1. Molar mass [86]:

$$
M_{m o l}=18.015 \cdot 10^{-3} \mathrm{kgmol}^{-1}
$$

2. Vapor specific heat at constant pressure (ambient conditions, [90]):

$$
C_{p}=1859.0 \mathrm{Jkg}^{-1} \mathrm{~K}^{-1}
$$

3. Critical temperature and pressure [86]:

$$
T_{c}=647.14 \mathrm{~K}, p_{c}=22.064 \cdot 10^{6} \mathrm{Nm}^{-2}
$$

4. Surface tension (in $\mathrm{Nm}^{-1}$, [90]):

$$
\begin{aligned}
\sigma=\sigma^{(1)} & \text { for } T \leq 249.39 K, \text { else: } \sigma=\sigma^{(2)}, \text { where: } \\
& \sigma^{(1)}=\left(0.1131283 \cdot 10^{-9}-0.3709125 \cdot 10^{-12} T\right) T^{4}-0.56464 \cdot 10^{-5} \\
\sigma^{(2)} & =76.1 \cdot 10^{-3}+0.155 \cdot 10^{-3}(273.15-T) .
\end{aligned}
$$

5. Liquid mass density (in $\mathrm{kgm}^{-3}$, see [91] and references therein):

$$
\begin{aligned}
\rho_{b}=\rho_{b}^{(1)} \text { for } T & <273.15 K, \text { else: } \rho_{b}=\rho_{b}^{(2)}, \text { where: } \\
\rho_{b}^{(1)} & =A_{L, 0}+A_{L, 1} T+A_{L, 2} T^{2} \\
\rho_{b}^{(2)} & =\frac{B_{L, 0}+B_{L, 1} T+B_{L, 2} T^{2}+B_{L, 3} T^{3}+B_{L, 4} T^{4}+B_{L, 5} T^{5}}{B_{L, 6}+B_{L, 7} T},
\end{aligned}
$$


with:

$A_{L, 0}=170.55 ; A_{L, 1}=5.9860 ; A_{L, 2}=-0.06458 ; B_{L, 0}=-2007.66 ; B_{L, 1}=$ $-13.0134 ; B_{L, 2}=0.184709 ; B_{L, 3}=-0.51251 \cdot 10^{-3} ; B_{L, 4}=0.6869 \cdot 10^{-6}$; $B_{L, 5}=-0.3933 \cdot 10^{-9} ; B_{L, 6}=-3.96032 ; B_{L, 7}=0.1816$.

6. Saturation pressure (in $\mathrm{Nm}^{-2}$, see [91] and references therein):

$$
p_{s}=\exp \left(A_{p, 1}+A_{p, 2} T+A_{p, 3} T^{2}+A_{p, 4} \ln (T)+\frac{A_{p, 5}}{T}\right)
$$

with:

$A_{p, 1}=21.125 ; A_{p, 2}=-2.7246 \cdot 10^{-2} ; A_{p, 3}=1.6853 \cdot 10^{-5} ; A_{p, 4}=2.4576 ;$ $A_{p, 5}=-6094.4642$.

7. Latent heat of condensation (in $\mathrm{Jkg}^{-1}$, for $200 \mathrm{~K} \leq T \leq 300 \mathrm{~K}$, [90]):

$$
L_{b}=L_{0}+L_{1} T,
$$

with $L_{0}=3105913.39 \mathrm{~J} / \mathrm{kg}$ and $L_{1}=-2212.97 \mathrm{~J} /(\mathrm{kgK})$

8. Binary diffusion coefficient of water vapor in air (in $\mathrm{m}^{2} \mathrm{~s}^{-1}$, see [58] and references therein):

$$
D_{v, a}=\frac{2.49}{p}\left(\frac{T}{295}\right)^{1.75}
$$

\section{A.2 Properties of heavy water $\left(\mathrm{D}_{2} \mathrm{O}\right)$}

All properties are taken from [110] and references therein.

1. Molar mass :

$$
M_{m o l}=20.027 \cdot 10^{-3} \mathrm{kgmol}^{-1}
$$

2. Vapor specific heat at constant pressure:

$$
C_{p}=1710.19 \mathrm{Jkg}^{-1} \mathrm{~K}^{-1}
$$

3. Critical temperature and pressure:

$$
T_{c}=643.89 \mathrm{~K}, p_{c}=21.66 \cdot 10^{6} \mathrm{Nm}^{-2}
$$

4. Surface tension (in $\mathrm{Nm}^{-1}$ ):

$$
\sigma=99.6635+0.009133 T^{\prime}-0.000275 T^{\prime 2}, \text { with: } T^{\prime}=1.022 T
$$


5. Liquid mass density (in $\mathrm{kgm}^{-3}$ ):

$\rho_{b}=0.09 \tanh (x)+0.847 \tau^{0.33}+0.338$, with:

$x=\frac{T-231}{51.5}$, and: $\tau=1-\frac{T}{T_{c}}$

6. Saturation pressure (in $\mathrm{Nm}^{-2}$ ):

$$
\begin{aligned}
& p_{s}=p_{c} \exp \left[\frac{T_{c}}{T}\left(a_{1} \tau+a_{2} \tau^{1.9}+a_{3} \tau^{2}+a_{4} \tau^{5.5}+a_{5} \tau^{10}\right)\right] \text {, with: } \\
& a_{1}=-7.815813 ; a_{2}=17.6012 ; a_{3}=-18.1747 ; a_{4}=-3.92488 ; a_{5}= \\
& 4.19174
\end{aligned}
$$

7. Latent heat of condensation (in $\mathrm{Jkg}^{-1}$ ):

$$
\begin{aligned}
& L_{b}=\frac{R_{u}}{M_{m o l}} T\left[\ln \left(\frac{p_{s}}{p_{c}}\right)+\phi\right], \text { with: } \\
& \phi=a_{1}+1.9 a_{2} \tau^{0.9}+2 a_{3} \tau+5.5 a_{4} \tau^{4.5}+10 a_{5} \tau^{9}
\end{aligned}
$$

\section{A.3 Properties of nitrogen $\left(\mathrm{N}_{2}\right)$}

1. Molar mass [44]:

$M_{m o l}=28.013 \cdot 10^{-3} \mathrm{kgmol}^{-1}$

2. Specific heat at constant pressure [44]:

$C_{p}=1039.66 \mathrm{Jkg}^{-1} \mathrm{~K}^{-1}$

\section{A.4 Properties of methane $\left(\mathrm{CH}_{4}\right)$}

1. Molar mass [86]:

$$
M_{m o l}=16.043 \cdot 10^{-3} \mathrm{kgmol}^{-1}
$$

2. Specific heat at constant pressure (ambient conditions):

$$
C_{p}=2224.65 \mathrm{Jkg}^{-1} \mathrm{~K}^{-1}
$$

\section{A.5 Properties of atmospheric air}

1. Molar mass [90]:

$$
M_{m o l}=28.96 \cdot 10^{-3} \mathrm{kgmol}^{-1}
$$

2. Specific heat at constant pressure (ambient conditions, [90]): 
$C_{p, a}=1004.0 \mathrm{Jkg}^{-1} \mathrm{~K}^{-1}$

3. Specific heat at constant volume (ambient conditions, [90]):

$C_{v, a}=716.96 \mathrm{Jkg}^{-1} \mathrm{~K}^{-1}$

\section{A.6 Properties of ethanol/hexanol mixture}

Properties used for test cases $1.1,1.2$ and $1.3(T=260.0 K$, see chapter 5 and [142]); index 1 refers to ethanol, index 2 to hexanol.

1. Molar masses:

$$
M_{m o l, 1}=46.07 \mathrm{kgmol}^{-1} ; M_{m o l, 2}=102.17 \mathrm{kgmol}^{-1}
$$

2. Pure component liquid mass densities:

$$
\rho_{b, 1}=817.5 \mathrm{kgm}^{-3} ; \rho_{b, 2}=845.4 \mathrm{kgm}^{-3}
$$

3. Pure component saturation pressures:

$$
p_{1}^{s, p}=598.36 \mathrm{Nm}^{-2} ; p_{2}^{s, p}=2.643 \mathrm{Nm}^{-2}
$$

4. Pure component surface tensions:

$$
\sigma_{1}=25.02 \cdot 10^{-3} \mathrm{Nm}^{-1} ; \sigma_{2}=28.90 \cdot 10^{-3} \mathrm{Nm}^{-1}
$$

5. Mixture saturation pressures (in $\mathrm{Nm}^{-2}, x_{1}$ is the molar fraction of ethanol in the liquid phase):

$$
p_{1}^{s}\left(x_{1}\right)=x_{1} p_{1}^{s, p} ; p_{2}^{s}\left(x_{1}\right)=\left(1-x_{1}\right) p_{2}^{s, p}
$$

6. Mixture surface tension (in $\mathrm{Nm}^{-1}$ ):

$$
\sigma=\left(25.02+7.31088\left(1-x_{1}\right)-3.43199\left(1-x_{1}\right)^{2}\right) \cdot 10^{-3}
$$

\section{A.7 Properties of ethanol/propanol mixture}

Properties used for test case 2 (see chapter 5); index 1 refers to ethanol, index 2 to propanol. All ethanol properties are taken from [128], and all propanol properties from [37].

1. Molar masses:

$$
M_{m o l, 1}=46.07 \mathrm{kgmol}^{-1} ; M_{m o l, 2}=60.10 \mathrm{kgmol}^{-1}
$$

2. Specific heats at constant pressure: 


$$
C_{p, v, 1}=1.5688 \cdot 10^{3} \mathrm{Jkg}^{-1} \mathrm{~K}^{-1} ; C_{p, v, 2}=1.4243 \cdot 10^{3} \mathrm{Jkg}^{-1} \mathrm{~K}^{-1}
$$

3. Pure component liquid mass densities (in $\mathrm{kgm}^{-3}$ ):

$$
\begin{aligned}
& \rho_{b, 1}=10^{3}\left(0.80625-84.5 \cdot 10^{-5}(T-273.15)+29.0 \cdot 10^{-8}(T-273.15)^{2}\right) ; \\
& \rho_{b, 2}=1.01077 \cdot 10^{3}-3.99649 \cdot 10^{-5} T-6.64923 \cdot 10^{-3} T^{2}+2.16751 \cdot 10^{-5} T^{3}- \\
& 2.46167 \cdot 10^{-8} T^{4} .
\end{aligned}
$$

4. Pure component saturation pressures (in $\mathrm{Nm}^{-2}$ ):

$$
\begin{aligned}
& p_{1}^{s, p}=133.322 \cdot 10^{16.87-(5819 / T)+\left(414300 / T^{2}\right)}, \text { for } T<219.1 K, \text { else: } \\
& p_{1}^{s, p}=133.322 \cdot 10^{9.760-(2371 / T)} ; \\
& p_{2}^{s, p}=133.322 \cdot \exp (150.248-(11286.5 / T)-19.19 \ln T)
\end{aligned}
$$

5. Pure component surface tensions (in $\mathrm{Nm}^{-1}$ ):

$$
\begin{aligned}
& \sigma_{1}=10^{-3} \cdot(23.97-0.085(T-273.15)) ; \\
& \sigma_{2}=25.28 \cdot 10^{-3}-8.394 \cdot 10^{-5}(T-273.15)
\end{aligned}
$$

6. Pure component latent heats of condensation (in $\mathrm{Jkg}^{-1}$ ):

$$
\begin{aligned}
L_{b, 1} & =4.184 \cdot 10^{3}\left(226.0-0.213(T-273.15)-0.00138(T-273.15)^{2}\right) ; \\
L_{b, 2} & =(8559.61-9.29 T) \frac{R_{u}}{M_{m o l, 2}}
\end{aligned}
$$

7. Mixture saturation pressures (in $\mathrm{Nm}^{-2}, x_{1}$ is the molar fraction of ethanol in the liquid):

$$
p_{1}^{s}\left(x_{1}\right)=x_{1} p_{1}^{s, p} ; p_{2}^{s}\left(x_{1}\right)=\left(1-x_{1}\right) p_{2}^{s, p}
$$

8. Mixture surface tension (in $\mathrm{Nm}^{-1}$ ):

$$
\sigma=x_{1} \sigma_{1}+\left(1-x_{1}\right) \sigma_{2}
$$

9. Mixture latent heat (in $\mathrm{Jkg}^{-1}, \mathrm{w}_{1}$ is the mass fraction of ethanol in the liquid phase):

$L=w_{1} L_{b, 1}+\left(1-w_{1}\right) L_{b, 2}$ 



\section{Classical NUCLEATION THEORY AND DROPLET GROWTH}

In the Classical Nucleation Theory (CNT), the nucleation rate is calculated by means of the following expression:

$$
J=K \exp \left(\frac{-\Delta G_{n^{*}}}{k_{B} T}\right)
$$

where, $K$ denotes the kinetic prefactor, and $\Delta G_{n^{*}}$ the critical Gibbs energy of droplet formation.

In the classical approach, the kinetic prefactor is given by:

$$
K=\frac{\rho_{v}^{2}}{\rho_{b}} \sqrt{\frac{2 \sigma}{\pi m_{1}^{3}}},
$$

and the critical Gibbs energy by:

$$
\Delta G_{n^{*}}=\Delta G_{C, n^{*}}=\frac{4}{3} \pi\left(r^{*}\right)^{2} \sigma .
$$

The critical radius $r^{*}$ is obtained from the relationship:

$$
r^{*}=\frac{2 \sigma}{\rho_{b} R_{v} T \ln S},
$$

The nucleation rate thus obtained is denoted by $J_{C N T}$. By subtracting the monomer formation enthalpy $\Delta G_{1}$ from $\Delta G_{n^{*}}$, the latter is corrected for the apparent inconsistency associated with monomer formation. The critical Gibbs energy thus becomes:

$$
\Delta G_{n^{*}}=\Delta G_{I C C T, n^{*}}=\frac{4}{3} \pi \sigma\left[\left(r^{*}\right)^{2}-r_{1}^{2}\right] .
$$

The nucleation rate obtained by means of Eq. (B.1), (B.2) and (B.5) is denoted by $J_{I C C T}$. It is noted that the CNT-model is consistent with using the Courtney equilibrium distribution to calculate the backward rate, whereas the ICCT-model is consistent with use of the SCC equilibrium distribution [127]. 
The fluid properties $\sigma$ and $\rho_{b}$, as well as the saturation pressure $p_{s}$ are all temperature dependent. Therefore, the nucleation rate effectively depends solely on the partial vapor pressure and the temperature: $J=J\left(p_{v}, T\right)$, or equivalently: $J=J(S, T)$.

In the CGDE, the droplet growth rate is determined by means of the Hertz-Knudsen growth law, which expresses the mass growth rate as:

$$
\dot{m}_{H K}=\frac{4 \pi r^{2} \alpha}{\sqrt{2 \pi R_{v} T}}\left(p_{v}-p_{s, r}\right) .
$$

The saturated vapor pressure $p_{s, r}$ over a curved surface with radius $r$ is given by the Kelvin equation:

$$
p_{s, r}=p_{s} \exp \left(\frac{2 \sigma}{r \rho_{b} R_{v} T}\right) .
$$

The Hertz-Knudsen growth law is a valid model when the Knudsen number $K n$ is larger than 2: $K n=l / 2 r>2$, with $l$ denoting the mean free path length of the vapor molecules. Closer inspection of the preceding equations again reveals that in addition to the droplet radius itself, only the temperature and saturation ratio are needed to determine $\dot{m}$. For sufficiently large droplets, it can be shown that $\dot{m}_{H K}=\dot{n} m_{1}$, where $\dot{n}$ is given by Eq. (3.19). 


\section{SUMMARY}

In this thesis, the problem of predicting the droplet size distribution in condensing flow is investigated numerically. The work is focused on two types of condensation problems: one where condensation occurs during the rapid transonic expansion of a compressible fluid, and a second one where condensation takes place in a synthetic turbulent flow field, reminiscent of atmospheric clouds.

A description is given of the thermodynamic model and governing transport equations for a two-phase liquid/vapor mixture in transonic condensing flow. The physical model is presented for the cases of non-isothermal and isothermal condensation, and for single- and multi-component condensation.

For single-component condensing flow, three master equations for the prediction of the droplet size distribution are evaluated: the kinetic equation (KE), and its first and second-order approximations, i.e. the general dynamic equation (GDE) and the Fokker-Planck equation (FPE), respectively. The evaluation is based on (a) the equilibrium distributions, (b) a nucleation pulse experiment, and (c) an expanding nozzle flow. Large differences are observed between the equilibrium distributions of the FPE and KE, whereas no equilibrium distribution exists for the GDE. For the nucleation pulse experiment, good agreement is found between the results of the KE, FPE and GDE, whereas for the condensing nozzle flow, the difference between the GDE- and the KE-distributions is significant. This is primarily due to the fact that quasi-steady nucleation takes place during the nucleation pulse experiment, whereas this is not the case for the nozzle flow. A sensitivity study of the KE-solution with respect to uncertainties in (a) the surface tension model, (b) the sticking probability, and (c) the equilibrium distribution, revealed that both the sticking probability and the equilibrium distribution have a significant influence on the predicted condensation-onset.

The research on single-component condensing flow is continued with an evaluation of the method of moments (MOM), which efficiently generates an approximate solution for the flow field and moments of the droplet size distribution. Two variants of the moment equations (ME) are considered: the ME derived from the kinetic equation (KE), and those derived from the general dynamic equation (GDE). Two well-known closure methods for the MOM are qualitatively evaluated: closure based 
on mean droplet size-approximations, and closure based on quadrature approximation. The evaluation is based on key requirements that have been identified from steady state and transient solutions of the KE. It is found that none of the closure methods perform satisfactorily, and that the quadrature approximations severely suffer from a lack of robustness. To quantify the accuracy of the MOM, a condensing nozzle flow is simulated. Using a benchmark solution obtained by simultaneously solving the fluid dynamics equations with the $\mathrm{KE}$ as a reference for comparison, it is demonstrated that the MOM can predict condensing flow with acceptable accuracy.

With respect to multi-component condensation, an evaluation is made of a condensing flow model, which utilizes a kinetic equation to predict the evolution of a two-component (binary) dispersion. The evaluation is based on a simulation of a nozzle flow experiment, for which measurement data are reported in the literature. A full two-way coupling between the thermodynamic variables and the binary droplet size distribution is employed, which is novel for such condensation problems. Comparison of predicted and measured temperatures and partial pressures at the onset of condensation shows a fair agreement of the theoretical predictions and experimental data. The full resolution of the binary droplet size distribution is exploited to verify whether a quasi-steady treatment of the nucleation process in condensing nozzle flow is warranted. For the test case considered, it is concluded that quasi-steady state theory is not valid, which suggests that caution should be exercised in analyzing the condensation in such flows by means of quasi-steady state theory. Finally, the strong sensitivity of the condensation model to the binary equilibrium distribution is revealed by comparing the nozzle flow field generated by using two different equilibrium size distributions in the computation of the backward rates in the kinetic equation.

Finally, the condensation of micro-droplets in a synthetic turbulent flow, reminiscent of atmospheric clouds, is investigated numerically and analytically. Droplets are followed through a synthetic turbulent flow field composed of random Fourier modes, with wave numbers ranging from the integral length scales $\left(O\left(10^{2} \mathrm{~m}\right)\right)$ to the Kolmogorov scales $\left(O\left(10^{-3} \mathrm{~m}\right)\right.$ ) that are typical for atmospheric clouds. Two fully Lagrangian droplet growth models are utilized: (a) a one-way coupled model in which only adiabatic cooling of a rising air parcel is considered, and (b) a two-way coupled model which also accounts for the effects of local vapor depletion and latent heat release. The simulations with the simplified model show that the droplet size distribution becomes broader in the course of time and resembles a Gaussian distribution. This result is supported by a theoretical analysis which relates the droplet surface area distribution to the dispersion of droplets in the turbulent flow. With respect to the two-way coupled model, it is shown that the predicted droplet size distributions are still very broad, despite the fact that the growth of droplets is stabilized by vapor depletion and latent heat release. 


\section{SAMENVATTING}

Dit proefschrift betreft het bepalen van de druppelgrootte verdelingsfunctie in condenserende stromingen. In het onderzoek worden twee specifieke typen van condenserende stromingen onderzocht, namelijk condensatie in een transsone expanderende gasstroom en condensatie in een synthetisch turbulent stromingsveld, welke de condensatie in atmosferische wolken nabootst.

Voor transsone condenserende stromingen wordt eerst het thermodynamisch model en de relevante transportvergelijkingen voor een twee-fasen fluïdum beschreven. Dit twee-fasen model wordt zowel voor isotherme als niet-isotherme condensatie gepresenteerd, alsook voor condensatie in één-component en multi-component systemen.

Voor stromingen met condensatie van één component wordt een evaluatie uitgevoerd van drie balansvergelijkingen waarmee de druppelgrootte verdelingsfunctie voorspeld kan worden, namelijk de zogenaamde "kinetic equation" (KE), de FokkerPlanck vergelijking (Fokker-Planck equation, FPE) en de "general dynamic equation" (GDE). De FPE en GDE zijn respectievelijk de tweede en eerste-orde benadering van de KE. De evaluatie wordt uitgevoerd aan de hand van (a) de evenwichtsdistributie, (b) een nucleatie-puls experiment en (c) een transsone condenserende kanaalstroming. Met betrekking tot de evenwichtsdistributie worden grote verschillen waargenomen tussen de resultaten van de KE en de FPE, terwijl er voor de GDE geen evenwichtsdistributie bestaat. De simulatie van het nucleatie-puls experiment toont een goede onderlinge overeenkomst tussen de resultaten verkregen met de KE, de FPE en de GDE, terwijl bij de kanaalstroming de verschillen relatief groot zijn. Dit is voornamelijk te verklaren uit het feit dat bij het nucleatie-puls experiment de druppelgrootte verdelingsfunctie een quasi-stationaire oplossing bereikt, terwijl dit bij de kanaalstroming niet het geval is. Vervolgens is een gevoeligheidsanalyse uitgevoerd om de invloed van (a) de oppervlaktespanning, (b) de waarschijnlijkheid voor opname van monomeren door druppels (zogenaamde "sticking probability") en (c) de evenwichtsverdeling, op de oplossing van de KE te quantificeren. Uit de resultaten blijkt dat vooral de oppervlaktespanning en de evenwichtsverdeling de KE-oplossing sterk beïnvloeden.

Voor stromingen met condensatie van één component is verder de momentenmen- 
thode ("method of moments", MOM) geëvalueerd, waarmee op efficiënte wijze een benaderende oplossing van het stromingsveld en de momenten van de druppelgrootte verdelingsfunctie kan worden voorspeld. Twee varianten van de momentvergelijkingen worden beschouwd: de eerste is afgeleid aan de hand van de KE en de tweede aan de hand van de GDE. Hiervoor zijn twee sluitingsmodellen gebruikt: één gebaseerd op gemiddelde druppelgroottes en een tweede gebaseerd op numerieke quadratuur. Deze sluitingsmodellen zijn getoetst aan de eisen die zijn afgeleid uit het stationaire en instationaire gedrag van de KE-oplossing. Uit deze evaluatie is gebleken dat geen van de beschouwde sluitingsmodellen aan deze eisen voldoen, waarbij tevens duidelijk is geworden dat de quadratuur-sluiting onvoldoende robuust is. Aan de hand van de simulatie van condensatie in een kanaalstroming is een kwantitatieve analyse gemaakt van de nauwkeurigheid van de MOM. Uit een vergelijking van de MOM-oplossing met een referentie-oplossing verkregen middels de KE, blijkt dat de MOM-resultaten redelijk nauwkeurig zijn .

Met betrekking tot stromingen met multi-componenten condensatie is een evaluatie uitgevoerd van een stromingsmodel dat in staat is om binaire (twee-componenten) condensatie te voorspellen. Dit model wordt getoetst aan de hand van een condenserende kanaalstroming waarvoor data in de literatuur beschikbaar is. De koppeling tussen de thermodynamische grootheden en de binaire druppelgrootte verdelingsfunctie is twee-zijdig, welke nieuw is voor dergelijke stromingen. Uit een vergelijking tussen de voorspelde en gemeten temperaturen en drukken bij het startpunt van condensatie in de kanaalstroming volgt dat het resultaat van het theoretisch model het experiment redelijk dicht benaderd. Aan de hand van de berekende oplossing voor de binaire druppelgrootte verdelingsfunctie is nagetrokken in hoeverre deze de quasistationaire oplossing benadert. Uit deze analyse is gebleken dat de quasi-stationaire oplossing niet bereikt wordt in de beschouwde kanaalstroming, wat aangeeft dat de toepasbaarheid van quasi-stationaire nucleatie theorie op dergelijke stromingen niet als vanzelfsprekend mag worden beschouwd. Een analyse van de gevoeligheid van de druppelgrootte verdelingsfunctie voor de keuze van de evenwichtsverdeling heeft verder aangetoond dat deze invloed sterk is.

Als laatste is een onderzoek uitgevoerd naar condensatie in een synthetisch turbulent stromingsveld, welke de condensatie in atmosferische wolken nabootst. Het synthetisch turbulent veld wordt verkregen door deze uit Fourier snelheidscomponenten met willekeurige amplitude, faseverschil en oriëntatie samen te stellen. Het gebruikte condensatiemodel is volledig Lagrangiaans van karakter, waarbij twee varianten zijn gebruikt: één waarbij de condensatie van druppels geen terugkoppeling heeft naar de thermodynamische toestandsvariabelen (het zogenaamde één-wegsmodel) en een tweede waarbij deze terugkoppeling wel in rekening wordt gebracht (zogenaamde twee-wegsmodel). Uit de resultaten verkregen met het één-wegsmodel blijkt dat de variantie van de druppelgrootte verdelingsfunctie toeneemt in de tijd en dat de 
verdelingsfunctie een Gaussische vorm aanneemt. Dit is in overeenstemming met een theoretische analyse waarin de toename van de variantie wordt gerelateerd aan turbulente dispersie. Voor wat betreft het twee-wegsmodel blijkt kwalitatief dat ook hier de variantie van de druppelgrootte verdelingsfunctie toeneemt, alhoewel de mate waarin dit gebuert veel minder is vanwege de terugkoppeling van de condensatie naar de temperatuur en verzadiging van de damp. 



\section{ACKNOWLEDGMENT}

The work presented here would not be possible without the efforts of a number of people who I am very much indebted to.

First of all I would like to express my sincere gratitude to my promotor Harry Hoeijmakers. I am grateful for the support and advice that you have given me during my $\mathrm{PhD}$-project. I especially appreciate your careful reading of the draft of this thesis, sparing no efforts in verifying the correctness of each detail.

I owe much gratitude to my daily supervisor and assistant-promotor Rob Hagmeijer. Rob, in these past four years we have taken a long journey through the field of physics and mathematics, during which your advice was indispensable. I especially enjoyed our discussions in front of the white board and your trademark approach of casting a problem into a clear mathematical form. I very much appreciate that you trusted me with the supervision of MSc-projects, and that I was able to practice my teaching skills during several work lectures.

During the past four years, I have had the pleasure of supervising the Master's projects of three Mechanical Engineering students. The first one was Dennis van Putten, who applied our numerical models to three-dimensional condensing flow, and even succeeded in extending these models to multi-component condensing flow during his stay at Twister B.V.. Dennis, I very much enjoyed our collaboration during your MSc-project and in the period thereafter when you started your career at Twister B.V. I especially appreciate your enthusiasm and the quality of work that you meticulously stick to. Perhaps the only weak point I noticed was that you had a hard time stomaching the Surinamese Nasi Moksi-meti dish we shared one evening!

Next, I had the pleasure of supervising Ulrich (Uli) Sachs, who was originally assigned the task to investigate the application of the quadrature method of moments to condensing flows. However, during the course of the project, this was changed to the evaluation of master equations in condensing flow. With a very critical approach to the subject, he managed to successfully complete his project, which earned him a rightful place as co-author on my first paper. Uli, I very much appreciate the important work that you contributed to, especially when considering that you endured severe personal hardship in the final stages of your project. 
Although not directly related to my PhD-project, I was also involved in supervising the MSc-project of Mark Klaassen, who investigated the optimization of condensation in nozzle flows. Mark, it was a great pleasure to be involved in your graduation project, where an important step was taken towards the implementation of our models in actual designs.

During a three-month visit at Newcastle University in Spring 2008, I had the pleasure of collaborating with Rutger IJzermans and Mike Reeks on the subject of cloud condensation. This was a very nice experience for me, both scientifically and socially. I owe much gratitude to Mike for having me over in his group, for taking part in the doctorate committee, and for contributing to our paper. I also am very grateful to Rutger for the many enjoyable and insightful discussions and his contribution to our paper, but also for showing me around in Newcastle, and for the very nice trip we took to the beautiful English countryside. Finally, I also would like to thank the other staff members, and PhD-students for their support and friendliness.

During this project there were several meetings with Rini van Dongen and Vincent Holten of Eindhoven Technical University, which provided crucial insight into the many problems that I encountered in my research. I wish to express my sincere gratitude to Rini and Vincent for sharing their knowledge and insights on the subject of condensation, and furthermore, I also thank Rini for taking part in my doctorate committee.

This project was initiated as part of the Spearhead Program on Dispersed Multiphase Flow by the Institute of Mechanics, Processes and Control Twente (IMPACT). I wish to express my sincere gratitude to IMPACT for funding of this project.

Although numerical work has many merits, I occasionally experienced its pitfalls in the form of computer rebellion. Fortunately, I could always count on Wouter den Breeijen to solve these problems in a fast and professional manner. I would also like to thank Anjenet Mettivier-Meyer for dealing with administrative matters during my $\mathrm{PhD}$-project, especially with regards to housing-accommodation on the campus. Furthermore, I am thankful to the rest of the Engineering Fluid Dynamics staff, fellow $\mathrm{PhD}$ students and MSc students for the enjoyable discussions over the past four years. Special thanks goes to my former room mates Remco Habing, Isaias HernandezRamirez, Rutger IJzermans, Mark van de Zande, Douwe Dros, Jacco Hospers and Ellen van Emden for giving me a pleasant work-environment.

I would like to express my sincere gratitude to my friends and relatives, both in Holland and Suriname, for their support and friendship.

Last, but certainly not the least, I would like to thank my parents, who continuously supported me throughout these years.

Enschede, August 2009,

Ryan Sidin 


\section{ABOUT THE AUTHOR}

Ryan Sidin was born on March 27, 1977, in Paramaribo, Suriname. He attended the 'Algemene Middelbare School' (AMS) in Paramaribo, from which he graduated in 1995. He then entered the BSc-program in Mechanical Engineering at the University of Suriname (UvS) and obtained his BSc-diploma in December 1999, graduating with distinction. In March 2000 he started as an assistant lecturer within the Department of Mechanical Engineering, where he was primarily tasked with courses related to fluid dynamics and heat transfer.

In March 2003 he was awarded a scholarship to continue his study at the Faculty of Science and Technology of Twente University, where he obtained his master degree in the Physics of Complex Fluids group in June 2004, under the supervision of dr. H.T.M. van den Ende and prof. dr. ir. W. Agterof. The subject of his master's thesis was the breakup of droplets in two-phase capillary flow.

In April 2005, he started as a PhD-student within the Engineering Fluid Dynamics group under the supervision of prof. dr. ir. H.T.M. Hoeijmakers and dr. ir. R. Hagmeijer at the University of Twente. His research focused on the prediction of droplet size distributions in condensing flow, which was part of the Spearhead Program on Dispersed Multi-phase Flows, facilitated by the Institute of Mechanics, Processes and Control-Twente (IMPACT). He collaborated with dr. ir. R. IJzermans and prof. M. Reeks on droplet condensation in atmospheric clouds during a three month-visit at Newcastle University in Spring 2008.

Parts of his work were presented at the $6^{\text {th }}$ International Conference on Multiphase Flow in Leipzig, Germany (2007), the EUROMECH fluid mechanics conference in Manchester, U.K. (2008), the $4^{\text {th }}$ IMS Turbulence Workshop at Imperial College, U.K. (2009), and at the $18^{\text {th }}$ ICNAA nucleation conference in Prague, Czech Republic (2009). 